\title{
Dysfunctions of the Lower Urinary Tract and Affective Symptoms
}

Citation for published version (APA):

Vrijens, D. M. J. (2017). Dysfunctions of the Lower Urinary Tract and Affective Symptoms. [Doctoral Thesis, Maastricht University]. Datawyse / Universitaire Pers Maastricht.

https://doi.org/10.26481/dis.20170706dv

Document status and date:

Published: 01/01/2017

DOI:

$10.26481 /$ dis.20170706dv

Document Version:

Publisher's PDF, also known as Version of record

\section{Please check the document version of this publication:}

- A submitted manuscript is the version of the article upon submission and before peer-review. There can be important differences between the submitted version and the official published version of record.

People interested in the research are advised to contact the author for the final version of the publication, or visit the DOI to the publisher's website.

- The final author version and the galley proof are versions of the publication after peer review.

- The final published version features the final layout of the paper including the volume, issue and page numbers.

Link to publication

\footnotetext{
General rights rights.

- You may freely distribute the URL identifying the publication in the public portal. please follow below link for the End User Agreement:

www.umlib.nl/taverne-license

Take down policy

If you believe that this document breaches copyright please contact us at:

repository@maastrichtuniversity.nl

providing details and we will investigate your claim.
}

Copyright and moral rights for the publications made accessible in the public portal are retained by the authors and/or other copyright owners and it is a condition of accessing publications that users recognise and abide by the legal requirements associated with these

- Users may download and print one copy of any publication from the public portal for the purpose of private study or research.

- You may not further distribute the material or use it for any profit-making activity or commercial gain

If the publication is distributed under the terms of Article $25 \mathrm{fa}$ of the Dutch Copyright Act, indicated by the "Taverne" license above, 
Dysfunctions of the Lower Urinary Tract and

Affective 8 symptoms

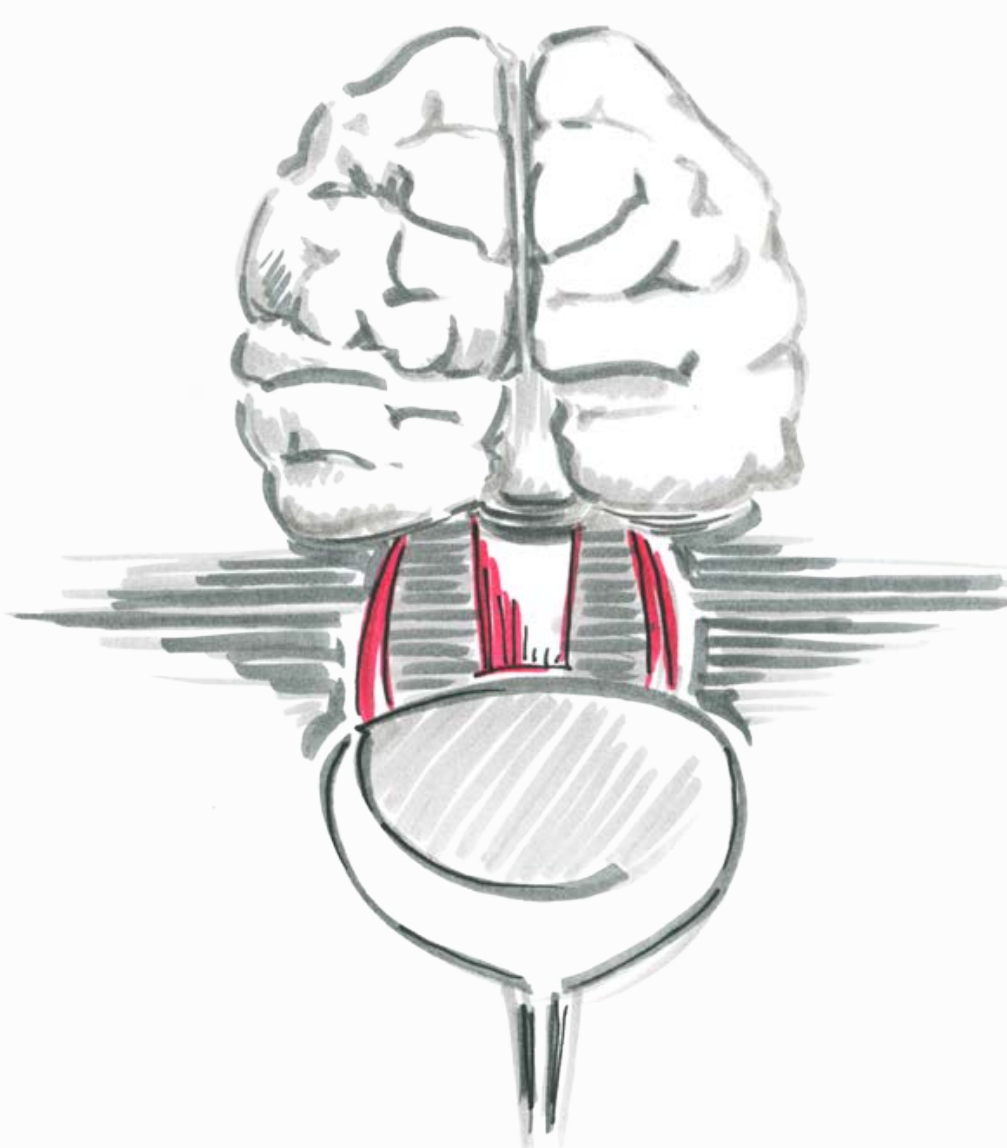

Désirée M.J.Vrijens 
(C) copyright Désirée MJ Vrijens, Maastricht 2017

Cover design and drawing: Gregorey Wens

Printing: Datawyse | Universitaire Pers Maastricht

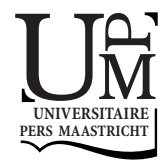

ISBN 9789461597076 


\title{
Dysfunctions of the Lower Urinary Tract and Affective Symptoms
}

\author{
Proefschrift \\ ter verkrijging van de graad van doctor aan de Universiteit Maastricht, \\ op gezag van de Rector Magnificus, Prof. dr. Rianne M. Letschert, \\ volgens het besluit van het College van Decanen, \\ in het openbaar te verdedigen \\ op donderdag 6 juli 2017 om 16.00 uur \\ door
}

Désirée Maria Josephina Vrijens 


\section{Promotores:}

Prof.dr. Ph.E.V. Van Kerrebroeck ( ${ }^{\mathrm{e}}$ promotor)

Prof. dr. G. A. van Koeveringe ( $2^{\mathrm{e}}$ promotor)

\section{Co-promotor:}

Dr. C. Leue

\section{Beoordelingscommissie:}

Prof.dr. H.W.M. Steinbusch (voorzitter)

Prof. dr. A.A.B. Lycklama à Nijeholt (Universiteit Leiden)

Prof.dr. J.G. Nijhuis

Prof.dr. De Ridder (Universiteit Leuven)

dr. J.J.M.H. Strik 


\section{Contents}

Chapter 1 General introduction

Chapter 2 Affective symptoms and the overactive bladder -

a systematic review

Journal of Psychosomatic Research 2015;78:95-108

Chapter 3 Prevalence of anxiety and depressive symptoms and their

65 association with pelvic floor dysfunctions - a cross sectional cohort study at a Pelvic Care Centre Neurourol and Urodyn. 2017 Febr 21, (Epub ahead of print)

Chapter 4 The relationship between self-consciousness/awareness and bladder sensations - a comparative study of patients with overactive bladder and healthy volunteers Accepted Low Urin Tract Symptoms

Chapter 5 Associations of psychometric affective parameters with urodynamic investigation for urinary frequency. Low Urin Tract Symptoms 2016 Jun 12, (Epub ahead of print)

Chapter 6 Screening for depression and anxiety in patients with storage or voiding dysfunction: A retrospective cohort study predicting outcome of sacral neuromodulation Neurourol Urodyn. 2016 Nov;35(8):1011-1016

Chapter 7 Patient reported outcome after treatment of urinary incontinence in a multidisciplinary pelvic care clinic Int J Urol 2015;22:1051-7

Chapter 8 General discussion

Summary

Nederlandse samenvatting

Valorisation

Dankwoord

Curriculum Vitae

Lijst van publicaties

List of abbreviations 

Chapter

1

General introduction 



\section{INTRODUCTION}

A Chinese proverb of 2000 years ago says: "The bladder is the mirror of the soul!". The bladder reflects our feelings, and wellbeing and we can all recognize that increased anxiety or stress can lead to subjective changes in voiding behaviour. Just before the start of a running a race there is a large queue of athletes in the restrooms, who have to void repeatedly. This suggests that psychological influences on voiding, which can lead to increased frequency and urgency, are also present in healthy individuals. In our clinical practice in the multi-disciplinary pelvic care clinic, we noticed a high rate of comorbidity between lower urinary tract dysfunction and affective symptoms. This intriguing observation led to this thesis.

\section{LOWER URINARY TRACT FUNCTION}

\section{Structure and function}

The lower urinary tract consists of the urinary bladder, the lower part of both ureters together with the ureterovesical junction, the urethra and external urethral sphincter, also referred to as the rhabdosphincter. In men the prostate is considered part of the urethra.

The bladder is a hollow organ that is situated within the pelvis. However the bladder can ascend even up to the umbilicus during filling. The bladder wall consists of five layers from inside out: the urothelium, the lamina propria with fibro-elastic connective tissue, the muscularis mucosa, the detrusor and the bladder serosa ${ }^{1}$. The muscle of the bladder, the detrusor, is composed of smooth muscle fibres, and consists of three layers. The fibres in the middle layer of the detrusor are arranged circular, and form an internal sphincter at the level of the bladder neck. The outer layer is composed of longitudinal fibres, that are thickest posteriorly at the bladder base ${ }^{1}$.

\section{Physiology and neuro-anatomy}

The function of the bladder is to store and to expel urine at a convenient time and place. In the adult, normal bladder capacity is approximately $400-500 \mathrm{ml}$, which results in a voiding frequency of 5-7 times a day, mostly at lower volumes. The predominant function of the bladder is that of a reservoir, to store urine at low pressures, even with large filling volumes. As a result of the visco-elastic properties of the bladder wall and the inhibition of filling phase detrusor contractions, the bladder is compliant, and the pressure inside of the bladder can remain low ${ }^{2}$. 
Voluntary micturition is a complex process and involves pathways at many levels of the brain, the spinal cord and the peripheral nervous system, and is mediated by multiple neurotransmitters.

The peripheral innervation of the lower urinary tract is derived from three sets of nerves: the hypogastic nerve, the pudendal nerve and the pelvic nerve, each with efferent and afferent components. Additionally, the innervation is both autonomic (parasympathetic and sympathetic) and somatic. The parasympathetic efferent innervation, (the pelvic nerves), originates from the spinal cord at S2, S3 and S4, and the postganglionic nerve fibres release acetylcholine, which stimulates M3 muscarinic receptors and leads to detrusor contraction. The sympathetic innervation originates in the T11-L2 segments, and runs through the inferior mesenteric ganglia and the hypogastric nerve, or through the paravertebral chain to the pelvic plexus at the base of the bladder and the urethra. The postganglionic fibres release noradrenaline that has either excitatory effects on the bladder base and urethra (contraction) through $\alpha$-receptors or inhibitory effects on the detrusor via $\beta 3$-receptors and so cause bladder relaxation. Finally, the somatic innervation, (nervus pudendus), arises from S2-S4 motor neurons in Onuf's nucleus, and supply the striated muscle of the external sphincter and pelvic floor, which is under voluntary control as well, through acetylcholine ${ }^{3-5}$.

\section{Afferent signalling from the bladder}

Afferent signals from the bladder are conveyed to the central nervous system by both sets of autonomic nerves. The sensation of bladder fullness goes to the spinal cord through the hypogastric and pelvic nerves. Additionally, sensory input from the bladder neck and urethra is passed through the pudendal and hypogastric nerves ${ }^{3}$. Afferent myelated $\mathrm{A} \delta$-fibres in the detrusor muscle respond to passive distension and active contraction, and are considered the primary mediators of bladder fullness ${ }^{6}$. Unmyelated C-fibres, found in the urothelium and lamina propria, are insensitive to bladder filling under physiological circumstances ${ }^{3,5}$.

The urothelium, lamina propria and myofibroblasts are recognized as important in bladder control. In response to bladder stretch, the urothelium releases a range of substances and hence plays an important role in bladder sensation ${ }^{7}$.

\section{Voiding reflex}

During filling of the bladder, the guarding reflects prevents involuntary micturition by inhibition of detrusor contraction and enhanced contraction of the urethral sphincter ${ }^{3}$. When the bladder is filled to a critical level of distension, the system switches from "off" to "on". This is a reflex pathway that involves the periaqueductal grey (PAG) in the midbrain, and the pontine micturition centre (PMC, also referred to as Barrington's nucle- 
us). The PMC activates a spinal efferent pathway to the sacral cord, to both inhibit the urethral sphincter and excite detrusor contraction, which results in voiding.

\section{Higher brain centres}

The voiding reflex is initiated by mechanical receptors in the bladder wall, and spontaneous voiding occurs whenever the bladder volume reaches a critical level. However, this reflex is under control of a supraspinal network, with the main function to prevent the voiding reflex to occur automatically. The voiding reflex is elicited when voiding is appropriate in time and place, and is under control of higher brain processes. The PAG plays an important role in this process by receiving afferent input from the bladder and passing these signals to higher brain centres. In addition, the PAG receives projections from the higher brain centres, and in particular the prefrontal cortex. When voiding is consciously desired, by the higher brain centres, then the PAG can stimulate the PMC, and so activate the voiding reflex ${ }^{3,5}$.

For volitional bladder control, a complex neural network of higher brain centres is involved with the suggestion of multiple parallel working circuits. One circuit includes the thalamus, the insula, the prefrontal cortex as well as the PAG. Information about the bladder is passed to the higher brain centres through the PAG. Bladder sensation is projected via the spinothalamic tracts to the hypothalamus and PAG ${ }^{8}$. The projections communicate in the thalamus and converge on the (non-dominant) insula, or insular cortex, which is regarded as the cortex that registers and maps visceral sensations ${ }^{9}$. During urine storage, insular activation has been observed ${ }^{10}$. In addition, significant brain activity in the insular cortex was seen in healthy women with an increasing urge to void $^{11}$.

The prefrontal cortex has extensive connections with the axis of the limbic systemhypothalamus and amygdala- as well as links with the insular cortex and the anterior cingulate cortex (ACC), and is involved in decision-making in a social and emotional context ${ }^{12}$. The prefrontal cortex is involved in voluntary bladder control, as seen in clinical studies in which nearly $70 \%$ of patients with a frontal stroke have urinary dysfunction ${ }^{13}$. Furthermore, patients with prefrontal damage have either transient or permanent incontinence, depending on the location of the damage ${ }^{14}$. In addition, decreased bladder sensation is associated with decreased perfusion of the frontal cortex ${ }^{15}$. Positron emission tomography (PET) studies of bladder control also point to involvement of the prefrontal cortex ${ }^{16-18}$. It has been stated that "the prefrontal cortex plays a role in making the decision about the appropriateness of the micturition to take place at that particular time and place" ${ }^{19}$. In other words the frontal cortex seems to be involved to provide the conscience of micturition.

The PAG plays an important role and has multiple motor projections into the ACC, which can be considered as the limbic motor cortex ${ }^{20}$. Functional imaging has demonstrated ACC activation during bladder filling ${ }^{21}$. The dorsal part of the ACC (dACC) is 
associated with the motivation to void, as it is strongly activated during further filling of a nearly full bladder in patients with urgency urinary incontinence (UUI) ${ }^{21}$. Furthermore, an increased response in the $\mathrm{dACC}$ is correlated with clinical severity of urgency incontinence ${ }^{22}$. This increased response in the dACC can also serve as a backup mechanism for continence, as the dACC is usually activated simultaneously with the adjacent supplementary motor cortex, where activation is associated with contraction of the pelvic floor and striated sphincter muscles ${ }^{23}$.

In addition, the parahippocampal/hypocampus/amygdala region could be of importance in bladder control as well. Limbic structures such as the amygdala, the PAG and hippocampus, which are closely linked with the prefrontal cortex, play an important role in primary emotions, such as anxiety and fear ${ }^{24}$. The parahippocampus is the grey matter that surrounds the hippocampus, and is part of the limbic system. Functional magnetic resonance imaging ( $\mathrm{fMRI}$ ) studies in older women with normal bladder function, revealed brain activation near the insula and dACC, when into an already full bladder, extra fluid was infused with an accompanying strong sensation. When infusing in a near-empty bladder and no apparent bladder sensation, these areas were not activated, but there was activation of the area of the PAG and the parahippocampal region ${ }^{25}$. This suggests unconscious monitoring of bladder signals. Changes in activation of a similar circuit has been observed after restoration of pathologically absent bladder sensation by SNM ${ }^{26}$. In addition, another study revealed in older women with overactive bladder $(O A B)$, differences in the activation pattern of the parahippocampal region, between women with $\mathrm{OAB}$ and detrusor overactivity (DO) compared to women with $\mathrm{OAB}$ without DO ${ }^{27}$. The precise function of this parahippocampal/hypocampus/amygdala region has to be further elucidated.

In summary, for volitional bladder control, a complex neural network of higher brain centres is involved, with the suggestion of parallel working circuits, among other regions, the insula, the cingulate and the frontal cortex (Figure 1). 


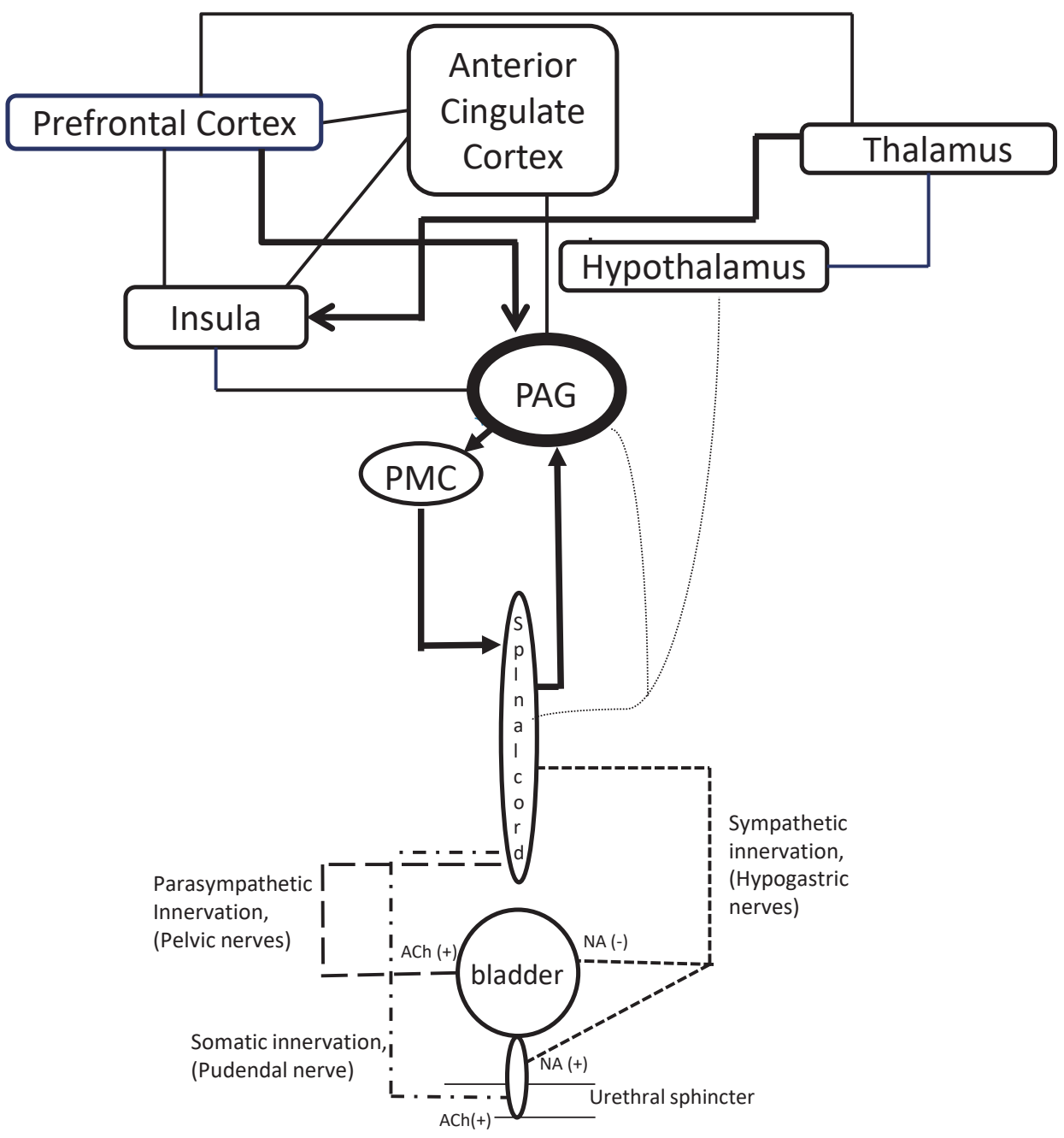

Figure 1. Bladder control, simplified schedule.

PAG=periaqueductal gray. $\mathrm{PMC}=$ pontine micturition centre. $\mathrm{NA}=$ Noradrenaline, $\mathrm{ACh}=$ acetylcholine, $(-)=$ inhibitory, $(+)=$ excitatory, The directional arrows of connectivity are mostly absent, intentionally, because this is yet to be elucidated. In order to simplify the parahippocampal/hypocampus/amygdala has been omitted from this figure. Reproduced and modified from ref 3.

\section{Neurotransmitters}

Various neurotransmitters are important in the central control of the lower urinary tract. There are excitatory and inhibitory transmitters, and some have both qualities, depending on the type of receptor that is activated. Dopamine has inhibitory effects and facilitatory effects on micturition ${ }^{3}$. Serotonin (5-HT) can facilitate voiding in rats, but there is more evidence in rats and cats that activation of the central serotonergic 
system, suppresses voiding, by increasing the efferent control of the urethra and inhibiting the excitatory parasympathetic input to the bladder ${ }^{28}$. Centrally, release of 5-HT by electrical stimulation of the brain stem in cats, can inhibit the micturition reflex ${ }^{29}$. However, recent work did not reveal significant changes in 5-HT levels at the PAG during the micturition reflex ${ }^{30}$.

\section{LOWER URINARY TRACT DYSFUNCTION}

\section{Overactive bladder}

Definition, prevalence, quality of life and health seeking behaviour

Overactive bladder (OAB) is defined by the International Continence Society (ICS) as a symptom complex of urgency, usually with frequency and nocturia (awakening at night to void), with ( $O A B$ wet) or without ( $O A B$ dry) urinary urgency incontinence. Urgency is the complaint of a sudden compelling desire to pass urine, which is difficult to defer ${ }^{31}$. It is estimated that approximately $16-17 \%$ of the adult population of the Western world is affected by $O A B{ }^{32} 33$. Incidence of $O A B$ wet and dry increase by age ${ }^{34-36}$, and because of the worldwide ageing of the population, the number of adults affected by UUI would increase ${ }^{37}$. The health care costs of $O A B$ are high ${ }^{38}$, and work productivity can be significantly impacted as well ${ }^{39}$. In addition, $O A B$ can be associated with comorbidity and increased mortality ${ }^{40}$. Furthermore, $O A B$ with and without incontinence has been associated with significantly lower quality of life scores compared to matched controls without voiding complaints ${ }^{34}$. It is of particular interest that $O A B$ does not only affect the patients, but has also has an impact on family members ${ }^{41}$. In general, the physician tends to underestimate the patients bother of urinary symptoms ${ }^{42}$.

Different coping strategies, such as timed voiding, locating toilets or avoiding drinking are used to cope with $O A B{ }^{43}$. The self-management of OAB leads almost inevitably to social withdrawal, sometimes relationship problems, fear in daily life, potentially leading to social and, psychological problems ${ }^{44}$. OAB can significantly affect daily life, and has been compared to other chronic illnesses. One article stated: "Patients are inclined to see other health problems as more serious than OAB. The role of that denial and avoidance of recognizing and labelling the problem, which means that the condition is experienced continually as 'raw', that is that the mental state of the sufferer resembles an 'open wound' leaving the sufferer experiencing unresolved loss which gradually destroys their life" ${ }^{44}$.

Although $O A B$ affects daily life and social relationships and has an adverse impact on quality of life, many individuals with $O A B$ symptoms do not bring their complaints to the attention of health care providers ${ }^{45}$. This is mainly due to either embarrassment ${ }^{43}$ or unawareness of medical treatment for $O A B{ }^{33}$. A very large study with 162.906 respondents in the United States reported that, of the individuals with probable overac- 
tive bladder symptoms, only less than half of them had discussed their symptoms with a medical care provider. In addition, only $22.5 \%$ had previously used medication for overactive bladder, and only $8.1 \%$ were currently on treatment ${ }^{46}$. In Europe, where 16776 interviews of people aged 40 and older were done in six countries, $60 \%$ of respondents with symptoms had consulted a doctor and only $27 \%$ were currently receiving treatment ${ }^{33}$. An inventory in a managed care organization revealed that 3 out of 4 patients with $O A B$ did not receive pharmacotherapy for their symptoms ${ }^{47}$.

\section{Pathophysiology of $O A B$ :}

$O A B$ can be caused by various underlying pathologies, but when there is no specific pathological disorder diagnosed, it is called idiopathic $O A B{ }^{31}$. The underlying pathophysiology of idiopathic OAB is multifactorial ${ }^{48,49}$. The ICS has classified the possible underlying pathological mechanisms of $O A B$ symptoms into: mechanisms underlying increased afferent activity and mechanisms involved in abnormal handling of afferent signals in the brain, the neurogenic hypothesis ${ }^{50}$.

Two probable theories contribute to the increased afferent activity. 1) The urothelium based hypothesis. 2) The myogenic based hypothesis ${ }^{50}$. Urothelial cells play an important role in modulating bladder responses to local chemical or mechanical stimuli. Up-regulation can lead to increased release of various chemical mediators and neurotransmitters, such as ATP, prostaglandins (PG), nerve growth factor (NGF), acetylcholine (ACh) and nitric oxic (NO). This in turn, may influence afferent nerve activity to generate $\mathrm{OAB}$ symptoms. The precise mechanism with which this interacts with neural tissue in order to get signal transduction, remains to be clarified ${ }^{51}$. The myogenic or motorsensory hypothesis states that locally synchronized micro contractions lead to increased afferent nerve activity. The micro contractions can occur because of increased excitability and coupling of myocytes ${ }^{50}$.

The neurogenic hypothesis suggests that damage of the central inhibitory pathways or sensitization of afferent nerves in the bladder can unmask primitive voiding reflexes that trigger bladder overactivity ${ }^{52}$. This is the case in neurological disease such as Parkinson, spinal cord lesions or stroke.

It is difficult to assess the pathophysiology of urgency. Urgency is a pathological sensation of a sudden compelling desire to pass urine, which is difficult to defer ${ }^{53}$. This should not be confused with the physiological urge sensation in a healthy person when the bladder is very full. Urgency is an important factor in the diagnosis of OAB. It can only be assessed in cognitively intact patients and there is no animal model available to study this. The precise mechanism of urgency, remains unknown ${ }^{54}$.

\section{Diagnosis of $O A B$.}

It is common to start the work-up of a patient with symptoms of OAB with an extensive history and a physical examination ${ }^{55,56}$. The use of a voiding diary was already reported in 1965, but only recently a 3 day voiding diary is validated for use in Lower Urinary 
Tract Symptoms (LUTS) ${ }^{57}$. Bladder diaries or voiding diaries are used to provide reliable measurements of urinary frequency and voided volumes ${ }^{56}$. Frequency volume charts (FVC) record voided volumes and time of micturition for a minimum of 24 hours, and voiding diaries include information on incontinence episodes, pad usage, fluid intake and degree of incontinence ${ }^{53,58}$. Sensation related bladder diaries (SR-BD) can measure bladder sensation in a non-invasive way. It has been proven to be a good tool to measure the bladder sensation during daily life. The degree of urgency can be evaluated by scoring the grade of perception of fullness of the bladder at each void ${ }^{59}$.

A urodynamic investigation can be used to reproduce symptoms, and allows direct assessment of lower urinary tract function by the measurement of relevant physiological parameters ${ }^{60}$. Urodynamics is the term used to describe a number of bladder function tests such as uroflowmetry and more invasive tests, such as filling cystometry and pressure flow study of voiding. Uroflowmetry is the measurement of a free flow void, and gives information about the rate and total amount of the flow. Furthermore, multichannel cystometry is the measurement of both bladder and intra-abdominal pressures by use of catheters inserted into the bladder and the rectum or vagina ${ }^{60}$. The term conventional urodynamic study (UDS) is most commonly used to describe cystometry. The European Guidelines on Urinary Incontinence recommends the use of urodynamics in urinary incontinence (UI) when its findings may potentially alter the choice of surgical treatment ${ }^{56}$. Other guidelines recommend to perform multichannel cystometry in women with incontinence and symptoms suggestive of $O A B{ }^{55}$. A study in 152 female patients with incontinence showed good to excellent reproducibility of same session repeated cystometry to detect stress urinary incontinence (SUI) and DO. Bladder volume at first sensation and maximum capacity were significantly higher during the second filling ${ }^{61}$. In a smaller study with 31 female patients with LUTS, the bladder capacity was also higher in the second run. Other urodynamic parameters were not statistically different, but showed great differences in individual patients ${ }^{62}$. When examining 30 healthy volunteers there was large test-retest variability with wide limits of agreement 63 . Similar findings were reported in 16 women who had two urodynamic investigations 6 weeks apart with relatively poor repeatability ${ }^{64}$.

DO is characterized by involuntary detrusor contractions, spontaneous or provoked, during the filling phase of a conventional-UDS ${ }^{58}$. It has been shown that OAB patients with DO have smaller maximum voided volumes, and significantly more incontinence episodes. There seems to be a difference in urodynamic data between patients with $\mathrm{OAB}$ and DO compared to those with $\mathrm{OAB}$ symptoms alone, as they were more likely to have abnormal sensation, with strong desire and urgency occurring at significantly lower bladder volumes ${ }^{65}$. A large study in females with $O A B$, with and without DO, showed that patients with DO had smaller functional bladder capacity and a higher urgency episode frequency. The presence of DO could be considered as a more severe form of $O A B{ }^{66}$. However, DO has also been found in healthy volunteers ${ }^{67}$. 


\section{Treatment of $O A B$}

Conservative treatment of $\mathrm{OAB}$ can consist of bladder training, in which patients are encouraged to gradually increase the interval between voiding episodes. When used in conjunction with a bladder diary, patients and clinicians can monitor progress through both the volumes voided, and the time interval between each void. It has been claimed in the past that up to $50 \%$ of patients will achieve long term benefit with this approach 68. A Cochrane review from 2012, comparing antimuscarinic drugs versus non-drug treatment for $\mathrm{OAB}$, concluded that in 7 small studies comparing different antimuscarinic drugs with bladder training, there has been no significant difference in cure rate. There was also no significant difference in frequency. The only difference was the subjective improvement: more people had no improvement with the drug therapy. When comparing other studies they concluded that antimuscarinics in combination with bladder training was better than bladder training alone or than antimuscarinics alone ${ }^{69}$.

The first choice of oral medical therapy for overactive bladder are antimuscarinic (anticholinergic) drugs ${ }^{56,70}$. The mechanism of action is through the muscarinic receptors in the bladder, although these receptors are present throughout the whole body. The most common side effect is dry mouth, but also constipation, blurred vision, fatigue and impaired cognitive function ${ }^{71}$. Nevertheless, the evidence regarding central nervous system (CNS) side effects of antimuscarinic drugs is not conclusive, as a systematic review reported that $77 \%(242 / 314)$ of clinical trials of antimuscarinic medication neither measured nor reported (adverse) CNS effect ${ }^{72}$. The adherence to antimuscarinic drugs is low, and more than half of the patients stop using the medication within the first three months, because of ineffectiveness, side effects or costs ${ }^{56}$. In addition, all studies with this type of drugs revealed considerable placebo effects of 9-38\% on the number of urgency episodes reported ${ }^{73}$. More recently, a beta 3 agonist is developed for the treatment of urgency, frequency and urgency incontinence ${ }^{74}$. The mechanism of action is inhibition of bladder afferent activity and consequently possible suppression of micro contractions of the bladder ${ }^{75}$. Long term (12 months) efficacy and safety of this drug has been described ${ }^{76}$. Intravesical injections of botulinum toxin can be given to patients with $O A B$ refractory to oral medication. Episodes of UUI, voiding frequency, urgency, and nocturia improved after the injection. Unfortunately the duration of the response is limited in time ${ }^{77,78}$. Early research focused on the motoric effect of botulinum toxin in reducing detrusor contractions by inhibition of neurotransmitter release from efferent fibres ${ }^{79}$. Nevertheless, botulinum toxin also seems to have a sensory effect in the bladder. In animal models it reduces neuropeptides release from sensory fibres in response to stimulation, and therefore leading to reduced afferent activity ${ }^{80}$. In addition, decreased levels of sensory receptors were found in bladder biopsies of patients treated with botulinum toxin ${ }^{81}$, and it is thought that botulinum toxin reduces afferent sensitization ${ }^{82}$.

Additionally, sacral neuromodulation (SNM) is a well-established option for overactive bladder complaints refractory to conservative treatment ${ }^{83}$. A permanent electrode 
is placed alongside a sacral nerve, mostly S3, through a sacral foramen. The implantation can be done under local anaesthesia together with fluoroscopic control ${ }^{84}$. At the first stage, of a two stage procedure ${ }^{85}$, the electrode is connected to a temporally external stimulator and the testing period of 2-4 weeks begins. When the patient has improved more than $50 \%$, as measured with voiding diaries during this testing phase, an internal pulse generator (IPG) is implanted during the second stage ${ }^{86}$. The exact mechanism of action is unknown but presumably SNM works by re-modelling the afferent nerve pathways ${ }^{83,87}$. SNM has central effects as well, as a PET study reported reversed midbrain responses after switching SNM on in women with urinary retention due to sphincter overactivity (Fowler syndrome) ${ }^{88}$. Kavia et al. showed changes in central activation on fMRI studies during SNM in patients with an inability to void. Nevertheless SNM seemed to act also directly at the sacral level ${ }^{26}$. Similar, regional cerebral blood flow measured by PET scan, was altered and different with acute SNM and chronic SNM ${ }^{89}$. SNM has been proven to be a successful therapy also at long term follow up ${ }^{90}$.

\section{Stress urinary incontinence}

Stress urinary incontinence (SUI) is defined as involuntary loss of urine associated with exertion, effort, sneezing or coughing ${ }^{58}$. It is a very common condition, although there is a wide range of published prevalence depending on definition and epidemiologic characteristics among the studies. A survey in the United States (US) found a prevalence of SUI at $23.7 \%$ among women. In Australia the prevalence of SUI was $16.8 \%{ }^{91,92}$. Generally, incontinence has been reported to occur in $5-69 \%$ of women, and in $1-39 \%$ of men ${ }^{93}$. Pregnancy and vaginal delivery are well known risk factors in women. In addition, there is a possible genetic influence for SUI, based on twin studies ${ }^{94}$. SUI occurs when the urethra may not remain closed during raised intra-abdominal pressure. This is caused by loss of vaginal or pelvic floor support to the urethra. Treatment is initially conservative with life style interventions and pelvic floor muscle training ${ }^{95}$. Surgical treatment follows in case of failure of conservative measures ${ }^{93}$. A recent study showed that treating SUI operatively in patients with depression was associated with significant improvement in quality of life and symptom burden ${ }^{96}$. Nevertheless, Zorn described no association between SUI and depression, only between idiopathic urinary incontinence and depression ${ }^{97}$.

\section{Lower urinary tract symptoms (LUTS)}

LUTS can be divided in storage, voiding or post-micturition symptoms. Traditionally these symptoms have been related to the prostate, causing bladder outlet obstruction $(\mathrm{BOO})^{53}$. However LUTS can be caused by various types of bladder dysfunctions, such as underactive bladder, overactive bladder, neurogenic disorders and urinary tract infections. It is a very common clinical complaint, particularly among men. The prevalence of 
LUTS increases with age, and can seriously alter quality of life ${ }^{98}$. For the assessment of symptoms and quality of life, validated symptom score questionnaires are recommended. The International Prostate Symptom Score (IPSS), containing seven symptom questions and a quality of life question, is the most commonly used in male patients with LUTS $^{99}$. Furthermore, urinalysis, FVC and uroflowmetry are standard investigations in the assessment of LUTS ${ }^{98}$. Treatment of LUTS depends on the underlying cause, and the nature of the most dominant symptom, and can be conservative, pharmacological or operative ${ }^{100}$.

\section{Underactive bladder}

Underactive bladder (UAB) is a common urinary tract dysfunction. UAB is characterized by a slow urine stream, hesitancy and straining to void, with or without feeling of incomplete bladder emptying and dribbling, often with storage symptoms. In contrast to $O A B$, it has been researched only very occasionally, and is poorly understood ${ }^{101}$. The clinical symptoms of this condition in the voiding phase are reduced urinary flow rate or post void residual, but are usually similar to symptoms of infra-vesical obstruction or dysfunctional voiding. Detrusor underactivity (DU) is a urodynamic diagnosis and is defined as a contraction of reduced strength and/or duration, resulting in prolonged bladder emptying and/or a failure to achieve complete bladder emptying within a normal time span (not yet published: working definition of the International Continent Society) The underactive bladder can be caused by an impaired detrusor muscle contractility, but defects in neuro-cognitive regulatory systems of bladder function can and reduced bladder sensation can be responsible for DU ${ }^{102}$. Initially DU was thought to be a (motor) contractility problem, nevertheless dysfunction of the central control of voiding can be impaired by afferent dysfunction ${ }^{103}$. Non-obstructive retention (NOR) as such refers to a condition in which no anatomical obstruction is present. NOR can be caused by DU, both through anatomical, reduced detrusor muscular content, as well as functional defects mentioned above.

Also functional causes such as detrusor sphincter dyssynergia, dysfunctional voiding and non-relaxing urethral sphincter obstruction can all be a responsible factor in NOR.

DU is a urodynamic diagnosis, and recently a nomogram has been developed to quantify the relationship between detrusor contractility and BOO in men with LUTS ${ }^{104}$. Presently, there is no adequate treatment for DU, and hence for underactive bladder, but it is likely that in the future pharmacological agents directed towards increasing the bladder contractility will become available ${ }^{102}$.

\section{Pelvic floor dysfunction}

Pelvic floor dysfunctions (PFDs) is defined by the International Urogynecology Association (IUGA) and the ICS as the signs or symptoms of pelvic organ prolapse (POP), UI, 
bladder storage, voiding, post micturition and sensory symptoms, lower urinary tract infections, lower urinary tract pain, sexual and ano-rectal dysfunction ${ }^{58}$. It is estimated that over the next 30 years there will be a $45 \%$ increase in demand for PFDs related healthcare due to rising public expectations, increasing prevalence of predisposing factors such as diabetes and obesity and ageing population ${ }^{105}$. Recently, the complexity of PFDs presented at our multidisciplinary pelvic floor centre, their multi-factorial character and frequent significant interactions between them has been reported ${ }^{106,107}$. It has been demonstrated that one out of every four female patients had more than one PF health problem ${ }^{106}$, in males this has been shown to be one out of every eight ${ }^{107}$. Due to the complexity of PFDs a multidisciplinary approach is advocated ${ }^{108}$.

\section{AFFECTIVE SYMPTOMS}

\section{Definition, prevalence and course}

Depression and anxiety are symptom based constructs, and classified as affective disorders according to the DSM-V psychiatric diagnostic criteria ${ }^{109}$. According to a survey of the World Health Organisation, anxiety is globally the most common psychiatric disorder ${ }^{110}$. For instance, the twelve-month prevalence of anxiety disorders in the US adult population is around $18 \%{ }^{110}$, and almost $10 \%$ for depression ${ }^{111}$. Additionally, the USlife time prevalence is approximately 30\% for anxiety disorders, and 20\% for depressive disorders ${ }^{112}$. However, according to the World Mental Health survey almost half of the people with serious complaints received no treatment in the twelve months prior to the diagnostic interview ${ }^{113}$. In general, the association of somatic diseases with a depressive disorder is not uncommon and affects $25 \%$ of the people in hospital in-patient populations ${ }^{114}$. For instance, in irritable bowel syndrome (IBS), a functional disorder of intestinal hypersensitivity and altered motility, the association with affective disorders is relatively clear, and IBS patients have been shown to have an approximately $60 \%$ higher odds to suffer from depression ${ }^{115}$. In addition, half of the population reporting a lifetime IBS diagnosis also had a lifetime mood or anxiety disorder ${ }^{116}$. Nevertheless psychiatric comorbidity regarding IBS is often unrecognized ${ }^{117}$. Moreover, at least in complex patients with medically unexplained functional complaints, psychiatric comorbidity is frequently under-detected ${ }^{118}$.

Mood disorders tend to be repetitive and chronic in course. One out of three patients may become chronically depressed, and nearly half of the patients remain to be undetected for years or inadequately controlled ${ }^{119}$. Furthermore, affective conditions complicate the prognosis of other chronic medical conditions ${ }^{120}$. Anxiety disorders for example, if left untreated, can lead to significant impairment of functioning, poor quality of life and considerable economic burden ${ }^{121}$. Moreover, mood disorders do not only affect the prognosis of comorbid somatic conditions, but also influence the level of health care consumption ${ }^{122}$. Furthermore, depression can adversely affect self-care, 
and increase the risk of new medical complaints, complications and mortality. Similarly, anxiety can amplify symptoms of medical illnesses, and worsen clinical outcomes ${ }^{123}$.

\section{Pathophysiology of affective symptoms}

Over decades several psychological models have been introduced to explain depression. A major shift in the understanding of depression became possible with the development of neurobiological techniques. Depletion or imbalance of norepinephrine ${ }^{124}$ or serotonin ${ }^{125}$ was connected to clinical depression, was shown in the sixties of the last century, and cholinergic-noradrenergic imbalance was detected in the early seventies 126. Impaired glucocorticoid and mineralocorticoid receptors gave a neuroendocrine explanation of depression ${ }^{127}$, given that stress activates the hypothalamus-pituitaryadrenal (HPA) axis. Corticotrophin releasing factor (CRF) is released from the hypothalamus to stimulate the pituitary gland to secrete adrenocorticotrophic hormone (ACTH), which in turns stimulates the secretion of glucocorticoids (cortisol). It has been suggested that the aetiology of depression is related to stress, since a first episode of depression occurs in people with vulnerability, stress-dependently. As the vulnerability increases, the level of stress needed to cause a depression, decreases. In addition, the occurrence of a depression episode increases the vulnerability ${ }^{128}$.

Modern psychobiology and psychiatry attempts to combine previous knowledge in a current final common pathway model, by linking experience and behaviour to the central nervous system. Psychological and biological etiological factors converge at midbrain mechanisms of pleasure and reward ${ }^{129}$. These limbic-diencephalic centres of emotional behaviour are complex. The limbic cortex is linked to the neocortex and its higher brain functions. The limbic cortex is in addition linked to the midbrain and lower brain centres, which are involved in autonomic function, hormonal function and sleep and wakefulness ${ }^{129}$.

The amygdala, insula and ACC are all dysfunctional in depression ${ }^{130}$. Additionally defects in function and morphology of the prefrontal cortex have been described ${ }^{128}$. Prolonged exposure to stress can cause damage to the prefrontal cortex, and the most stress-sensitive region is the ACC ${ }^{131}$.

In anxiety disorders, there is an increased activation in the sensory processing regions of the brain, such as the occipital cortex and the thalamus ${ }^{132}$. Additionally, increased activity has been seen in emotion-generating regions such as the amygdala, the insula and the dACC. The regulatory regions in the hippocampus and prefrontal cortex are hypo-active ${ }^{133}$. Recent literature suggests decreased connectivity between emotion-generating and emotion-modulating regions to be a factor in the pathogenesis of anxiety $^{134}$.

\section{Screening tool for affective symptoms}

In this thesis we used the Hospital Anxiety and Depression Scale (HADS) ${ }^{135}$. The HADS is a short, self-reported scale to screen for the presence of a mood and anxiety complaints 
in medically ill patients. It contains 14 items which are divided into two subscales, that assess depression (HADS-D) and anxiety (HADS-A). The items are rated on 4-point Likert type scales. The HADS has a high internal consistency (Cronbach's alpha $=0.85$ ). The HADS can be used as a screening instrument in the hospital setting, as well as in primary care for screening and for monitoring of affective changes over time. The results of the HADS-D and HADS-A subscales can be dichotomized with a cut-off point of 8 (normal vs. abnormal depressive symptoms or anxiety) or a cut-off point of 11 (normal or mild vs. moderate or severe depressive symptoms or anxiety). It can also be categorized as a four level ordinal variable: normal 0-7; mild depressive symptoms or anxiety 8-10, moderate depressive symptoms or anxiety 11-14 or severe depressive symptoms or anxiety $15-21^{135}$

\section{ASSOCIATION OF LOWER URINARY TRACT DYSFUNCTION AND AFFECTIVE SYMPTOMS.}

Medical literature described the relationship between psychiatric diseases and bladder function disorders already in 1900 when Bierhoff presented a case of "bladder neurosis" ${ }^{136}$. In 1949 "disturbances of bladder function" in relation to emotional states were described. Bladder problems have been described to be associated with anxiety and resentment accompanying conflict ${ }^{137}$. These ideas have their roots in the theories of neurosis, initiated by Sigmund Freud and Carl Jung of the end of the $19^{\text {th }}$ century. Bladder complaints were considered to be covered functional sexual disorders, resulting in depression, unconscious anger and helplessness, rather than a urological disorder ${ }^{138}$. In 1964 a review regarding "uropsychiatry" was published ${ }^{139}$.

Presently, there is renewed interest in this matter, reflected by an increased amount of publications found in PubMed over the last few years. The importance of the link between lower urinary tract and psychological symptoms was emphasized in 2011. The International Consultation on Incontinence-Research Society (ICI-RS) organized a thinkthank session on the subject ${ }^{140}$. Nevertheless, solid evidence about the association is lacking.

Are patients with bladder complaints vulnerable to affective complaints due to embarrassment, social isolation and functional impairment? Or is there another cause for the association? Is lower urinary tract dysfunction solely a problem of the lower urinary tract? Or is dysfunction due to altered signalling and processing information from the bladder in the higher brain centres?

The aim of this thesis is to assess whether there is an association between lower urinary tract dysfunction and affective symptoms and vice-versa. Furthermore, we want to elaborate on what may be the nature of this association. 


\section{OUTLINE OF THIS THESIS}

The general introduction presented in Chapter 1 gives a brief overview of the anatomy and physiology of the lower urinary tract. Additionally lower urinary tract dysfunction, in particular overactive bladder, and affective symptoms are described. A systematic review of the literature concerning the association between overactive bladder and affective symptoms is displayed in Chapter 2. The prevalence of anxiety and depressive symptoms and their association with pelvic floor dysfunction is presented in Chapter 3. Chapter 4 shows the results of a prospective study concerning differences in selfawareness, self-consciousness, depression and anxiety scores and SR-BD between healthy volunteers and patients with OAB. In addition, the associations of HADS scores with urodynamic investigations in patients with urinary frequency are presented in Chapter 5. Chapter 6 assesses whether the presence of affective symptoms is related to treatment outcome of SNM. Patient reported outcome regarding urinary incontinence is presented in Chapter 7. To conclude, a general discussion of all the studies presented in this thesis is given in Chapter 8. 


\section{REFERENCES}

1. de Groat, W.C., Anatomy and physiology of the lower urinary tract. Urol Clin North Am, 1993. 20(3): p. 383-401.

2. Andersson, K.E., Pathways for relaxation of detrusor smooth muscle. Adv Exp Med Biol, 1999. 462: p. 241-52.

3. Fowler, C.J., D. Griffiths, and W.C. de Groat, The neural control of micturition. Nat Rev Neurosci, 2008. 9(6): p. 453-466.

4. Roberts, M.M., Neurophysiology in neurourology. Muscle Nerve, 2008. 38(1): p. 815-36.

5. Chapple, C., Chapter 2: Pathophysiology of neurogenic detrusor overactivity and the symptom complex of "overactive bladder". Neurourol Urodyn, 2014. 33 Suppl 3: p. S6-13.

6. Habler, H.J., W. Janig, and M. Koltzenburg, Activation of unmyelinated afferent fibres by mechanical stimuli and inflammation of the urinary bladder in the cat. J Physiol, 1990. 425: p. 545-62.

7. Andersson, K.E. and A. Arner, Urinary bladder contraction and relaxation: physiology and pathophysiology. Physiol Rev, 2004. 84(3): p. 935-86.

8. Craig, A.D., How do you feel? Interoception: the sense of the physiological condition of the body. Nat Rev Neurosci, 2002. 3(8): p. 655-66.

9. Craig, A.D., Interoception: the sense of the physiological condition of the body. Curr Opin Neurobiol, 2003. 13(4): p. 500-5.

10. Andersson, K.E., Bladder activation: afferent mechanisms. Urology, 2002. 59(5 Suppl 1): p. 43-50.

11. Kuhtz-Buschbeck, J.P., et al., Cortical representation of the urge to void: a functional magnetic resonance imaging study. J Urol, 2005. 174(4 Pt 1): p. 1477-81.

12. Damasio, A.R., The somatic marker hypothesis and the possible functions of the prefrontal cortex. Philos Trans R Soc Lond B Biol Sci, 1996. 351(1346): p. 1413-20.

13. Sakakibara, R., C.J. Fowler, and T. Hattori, Voiding and MRI analysis of the brain. Int Urogynecol J Pelvic Floor Dysfunct, 1999. 10(3): p. 192-9.

14. Mochizuki, H. and H. Saito, Mesial frontal lobe syndromes: correlations between neurological deficits and radiological localizations. Tohoku J Exp Med, 1990. 161 Suppl: p. 231-9.

15. Griffiths, D., Clinical studies of cerebral and urinary tract function in elderly people with urinary incontinence. Behav Brain Res, 1998. 92(2): p. 151-5.

16. Blok, B.F., A.T. Willemsen, and G. Holstege, A PET study on brain control of micturition in humans. Brain, 1997. 120 ( Pt 1): p. 111-21.

17. Athwal, B.S., et al., Brain responses to changes in bladder volume and urge to void in healthy men. Brain, 2001. 124(Pt 2): p. 369-77.

18. Nour, S., et al., Cerebral activation during micturition in normal men. Brain, 2000. 123 ( Pt 4): p. 781-9.

19. Blok, B.F., Central pathways controlling micturition and urinary continence. Urology, 2002. 59(5 Suppl 1): p. 13-7.

20. Devinsky, O., M.J. Morrell, and B.A. Vogt, Contributions of anterior cingulate cortex to behaviour. Brain, 1995. 118 ( Pt 1): p. 279-306.

21. Griffiths, D. and S.D. Tadic, Bladder control, urgency, and urge incontinence: evidence from functional brain imaging. Neurourol Urodyn, 2008. 27(6): p. 466-74.

22. Tadic, S.D., et al., Brain activity measured by functional magnetic resonance imaging is related to patient reported urgency urinary incontinence severity. J Urol, 2010. 183(1): p. 221-8.

23. Kuhtz-Buschbeck, J.P., et al., Activation of the supplementary motor area (SMA) during voluntary pelvic floor muscle contractions--an fMRI study. Neuroimage, 2007. 35(2): p. 449-57.

24. Apps, R. and P. Strata, Neuronal circuits for fear and anxiety - the missing link. Nat Rev Neurosci, 2015. 16(10): p. 642.

25. Tadic, S.D., et al., Brain responses to bladder filling in older women without urgency incontinence. Neurourol Urodyn, 2013. 32(5): p. 435-40. 
26. Kavia, R., et al., A functional magnetic resonance imaging study of the effect of sacral neuromodulation on brain responses in women with Fowler's syndrome. BJU Int, 2010. 105(3): p. 366-72.

27. Tadic, S.D., et al., Brain activity underlying impaired continence control in older women with overactive bladder. Neurourol Urodyn, 2012. 31(5): p. 652-8.

28. de Groat, W.C., Influence of central serotonergic mechanisms on lower urinary tract function. Urology, 2002. 59(5 Suppl 1): p. 30-6.

29. McMahon, S.B., J.F. Morrison, and K. Spillane, An electrophysiological study of somatic and visceral convergence in the reflex control of the external sphincters. J Physiol, 1982. 328: p. 379-87.

30. Kitta, T., et al., Differences in neurotransmitter systems of ventrolateral periaqueductal gray between the micturition reflex and nociceptive regulation: An in vivo microdialysis study. Int J Urol, 2016. 23(7): p. 593-8.

31. Abrams, P., et al., The standardisation of terminology in lower urinary tract function: report from the standardisation sub-committee of the International Continence Society. Urology, 2003. 61(1): p. 37-49.

32. Coyne, K.S., et al., National community prevalence of overactive bladder in the United States stratified by sex and age. Urology, 2011. 77(5): p. 1081-7.

33. Milsom, I., et al., How widespread are the symptoms of an overactive bladder and how are they managed? A population-based prevalence study. BJU Int, 2001. 87(9): p. 760-6.

34. Stewart, W.F., et al., Prevalence and burden of overactive bladder in the United States. World J Urol, 2003. 20(6): p. 327-36.

35. Irwin, D.E., et al., Population-Based Survey of Urinary Incontinence, Overactive Bladder, and Other Lower Urinary Tract Symptoms in Five Countries: Results of the EPIC Study. European Urology, 2006. 50(6): p. 1306-1315.

36. Coyne, K.S., et al., The prevalence of lower urinary tract symptoms (LUTS) in the USA, the UK and Sweden: results from the Epidemiology of LUTS (EpiLUTS) study. BJU Int, 2009. 104(3): p. 352-60.

37. Irwin, D.E., et al., Worldwide prevalence estimates of lower urinary tract symptoms, overactive bladder, urinary incontinence and bladder outlet obstruction. BJU Int, 2011. 108(7): p. 1132-8.

38. Hu, T.W., et al., Estimated economic costs of overactive bladder in the United States. Urology, 2003. 61(6): p. 1123-8.

39. Sexton, C.C., et al., Impact of overactive bladder on work productivity in the United States: results from EpiLUTS. Am J Manag Care, 2009. 15(4 Suppl): p. S98-S107.

40. Foley, A.L., et al., Association between the Geriatric Giants of urinary incontinence and falls in older people using data from the Leicestershire MRC Incontinence Study. Age Ageing, 2012. 41(1): p. 35-40.

41. Coyne, K.S., L.S. Matza, and J. Brewster-Jordan, "We have to stop again?!": The impact of overactive bladder on family members. Neurourology and Urodynamics, 2009. 28(8): p. 969-975.

42. Rodriguez, L.V., et al., Discrepancy in patient and physician perception of patient's quality of life related to urinary symptoms. Urology, 2003. 62(1): p. 49-53.

43. Ricci, J.A., et al., Coping strategies and health care-seeking behavior in a US national sample of adults with symptoms suggestive of overactive bladder. Clin Ther, 2001. 23(8): p. 1245-59.

44. Nicolson, P., et al., It's just the worry about not being able to control it! A qualitative study of living with overactive bladder. Br J Health Psychol, 2008. 13(Pt 2): p. 343-59.

45. Epstein, L.B. and R.P. Goldberg, The overactive bladder and quality of life. International journal of fertility and women's medicine, 2005. 50(1): p. 30-36.

46. Benner, J.S., et al., Bother related to bladder control and health care seeking behavior in adults in the United States. J Urol, 2009. 181(6): p. 2591-8.

47. Randolph, M.F. and M. Greenfield, Lower urinary tract obstruction in normal male children. Early detection by urinary diary. Am J Dis Child, 1965. 110(5): p. 523-30.

48. Roosen, A., et al., A Refocus on the Bladder as the Originator of Storage Lower Urinary Tract Symptoms: A Systematic Review of the Latest Literature. Eur Urol, 2009. 56(5): p. 810-9.

49. Schick, E., et al., Frequency-volume chart: the minimum number of days required to obtain reliable results. Neurourol Urodyn, 2003. 22(2): p. 92-6. 


\section{Chapter 1}

50. Koelbl H, I.T., Salvatore S, Laterza R.M, Lowry A, Sievert K-D, Sultan A., Pathofysiology of urinary incontinence, faecal incontinence and pelvic organ prolapse, in Incontinence 5th Edition. ICUD-EAU, C.L. Abrahams P, Khoury S, Wein A., Editor. 2013. p. 263-360.

51. Yoshida, M., et al., The forefront for novel therapeutic agents based on the pathophysiology of lower urinary tract dysfunction: pathophysiology and pharmacotherapy of overactive bladder. J Pharmacol Sci, 2010. 112(2): p. 128-34.

52. de Groat, W.C., A neurologic basis for the overactive bladder. Urology, 1997. 50(6A Suppl): p. 36-52; discussion 53-6.

53. Abrams, P., et al., The standardisation of terminology of lower urinary tract function: report from the Standardisation Sub-committee of the International Continence Society. Neurourol Urodyn, 2002. 21(2): p. 167-78.

54. Michel, M.C. and C.R. Chapple, Basic mechanisms of urgency: preclinical and clinical evidence. Eur Urol, 2009. 56(2): p. 298-307.

55. National Institute of Clinical Excellence (NICE). CG171 Urinary Incontinence in Women: Full Guideline. 2013.

56. Lucas MG, B.D., et al. EAU Guidelines on Urinary Incontinence. 2014; Available from: http://www.uroweb.org/guidelines/online-guidelines/.

57. Bright, E., et al., Developing and validating the International Consultation on Incontinence Questionnaire bladder diary. Eur Urol, 2014. 66(2): p. 294-300.

58. Haylen, B.T., et al., An International Urogynecological Association (IUGA)/International Continence Society (ICS) joint report on the terminology for female pelvic floor dysfunction. Neurourol Urodyn, 2010. 29(1): p. 4-20.

59. De Wachter, S. and J.J. Wyndaele, Frequency-volume charts: a tool to evaluate bladder sensation. Neurourol Urodyn, 2003. 22(7): p. 638-42.

60. Schäfer, W., et al., Good urodynamic practices: Uroflowmetry, filling cystometry, and pressure-flow studies.. Neurourology and Urodynamics, 2002. 21(3): p. 261-274.

61. Broekhuis, S.R., et al., Reproducibility of same session repeated cystometry and pressure-flow studies in women with symptoms of urinary incontinence. Neurourol Urodyn, 2010. 29(3): p. 428-31.

62. Mortensen, S., G. Lose, and H. Thyssen, Repeatability of cystometry and pressure-flow parameters in female patients. Int Urogynecol J Pelvic Floor Dysfunct, 2002. 13(2): p. 72-5.

63. Brostrom, S., P. Jennum, and G. Lose, Short-term reproducibility of cystometry and pressure-flow micturition studies in healthy women. Neurourol Urodyn, 2002. 21(5): p. 457-60.

64. Gupta, A., G. Defreitas, and G.E. Lemack, The reproducibility of urodynamic findings in healthy female volunteers: results of repeated studies in the same setting and after short-term follow-up. Neurourol Urodyn, 2004. 23(4): p. 311-6.

65. Guralnick, M.L., et al., Objective differences between overactive bladder patients with and without urodynamically proven detrusor overactivity. Int Urogynecol J, 2010. 21(3): p. 325-9.

66. Giarenis, I., et al., Is there a difference between women with or without detrusor overactivity complaining of symptoms of overactive bladder? BJU Int, 2013. 112(4): p. 501-7.

67. van Waalwijk van Doorn, E.S., A. Remmers, and R.A. Janknegt, Conventional and extramural ambulatory urodynamic testing of the lower urinary tract in female volunteers. J Urol, 1992. 147(5): p. 1319-25; discussion 1326.

68. Holmes, D.M., et al., Bladder training-3 years on. Br J Urol, 1983. 55(6): p. 660-4.

69. Rai, B.P., et al., Anticholinergic drugs versus non-drug active therapies for non-neurogenic overactive bladder syndrome in adults. Cochrane Database Syst Rev, 2012. 12: p. CD003193.

70. Shamliyan, T., J. Wyman, and R.L. Kane, in Nonsurgical Treatments for Urinary Incontinence in Adult Women: Diagnosis and Comparative Effectiveness. 2012, april, Agency for Healthcare Research and Quality (US): Rockville (MD).

71. Farmacotherapeutisch kompas. http://www.farmacotherapeutischkompas.nl. 
72. Paquette, A., P. Gou, and C. Tannenbaum, Systematic review and meta-analysis: do clinical trials testing antimuscarinic agents for overactive bladder adequately measure central nervous system adverse events? J Am Geriatr Soc, 2011. 59(7): p. 1332-9.

73. Michel, M.C. and J.J. de la Rosette, Role of muscarinic receptor antagonists in urgency and nocturia. BJU Int, 2005. 96 Suppl 1: p. 37-42.

74. Bhide, A.A., et al., Mirabegron - a selective beta3-adrenoreceptor agonist for the treatment of overactive bladder. Res Rep Urol, 2012. 4: p. 41-5.

75. Aizawa, N., Y. Homma, and Y. Igawa, Effects of mirabegron, a novel beta3-adrenoceptor agonist, on primary bladder afferent activity and bladder micro contractions in rats compared with the effects of oxybutynin. Eur Urol, 2012. 62(6): p. 1165-73.

76. Chapple, C.R., et al., Randomized double-blind, active-controlled phase 3 study to assess 12-month safety and efficacy of mirabegron, a beta(3)-adrenoceptor agonist, in overactive bladder. Eur Urol, 2013. 63(2): p. 296-305.

77. Mangera, A., et al., Contemporary management of lower urinary tract disease with botulinum toxin A: a systematic review of botox (onabotulinumtoxinA) and dysport (abobotulinumtoxinA). Eur Urol, 2011. 60(4): p. 784-95.

78. Duthie, J.B., et al., Botulinum toxin injections for adults with overactive bladder syndrome. Cochrane Database Syst Rev, 2011(12): p. CD005493.

79. Chancellor, M.B., et al., Drug Insight: biological effects of botulinum toxin A in the lower urinary tract. Nat Clin Pract Urol, 2008. 5(6): p. 319-28.

80. Meng, J., et al., Synaptobrevin I mediates exocytosis of CGRP from sensory neurons and inhibition by botulinum toxins reflects their anti-nociceptive potential. J Cell Sci, 2007. 120(Pt 16): p. 2864-74.

81. Apostolidis, A., et al., Decreased sensory receptors P2X3 and TRPV1 in suburothelial nerve fibers following intradetrusor injections of botulinum toxin for human detrusor overactivity. J Urol, 2005. 174(3): p. $977-$ 82; discussion 982-3.

82. Kanai, A., et al., Researching bladder afferents-determining the effects of beta(3) -adrenergic receptor agonists and botulinum toxin type-A. Neurourol Urodyn, 2011. 30(5): p. 684-91.

83. Van Kerrebroeck, P.E., Advances in the role of sacral nerve neuromodulation in lower urinary tract symptoms. Int Urogynecol J, 2010. 21 Suppl 2: p. S467-74.

84. Spinelli, M., et al., New sacral neuromodulation lead for percutaneous implantation using local anesthesia: description and first experience. J Urol, 2003. 170(5): p. 1905-7.

85. Janknegt, R.A., E.H. Weil, and P.H. Eerdmans, Improving neuromodulation technique for refractory voiding dysfunctions: two-stage implant. Urology, 1997. 49(3): p. 358-62.

86. Spinelli, M. and K.D. Sievert, Latest technologic and surgical developments in using InterStim Therapy for sacral neuromodulation: impact on treatment success and safety. Eur Urol, 2008. 54(6): p. 1287-96.

87. Amend, B., et al., How does neuromodulation work. Neurourol Urodyn, 2011. 30(5): p. 762-5.

88. Dasgupta, R., et al., Changes in brain activity following sacral neuromodulation for urinary retention. J Urol, 2005. 174(6): p. 2268-72.

89. Blok, B.F., et al., Different brain effects during chronic and acute sacral neuromodulation in urge incontinent patients with implanted neurostimulators. BJU Int, 2006. 98(6): p. 1238-43.

90. Herbison, G.P. and E.P. Arnold, Sacral neuromodulation with implanted devices for urinary storage and voiding dysfunction in adults. Cochrane Database Syst Rev, 2009(2): p. CD004202.

91. Minassian, V.A., W.F. Stewart, and G.C. Wood, Urinary incontinence in women: variation in prevalence estimates and risk factors. Obstet Gynecol, 2008. 111(2 Pt 1): p. 324-31.

92. Botlero, R., et al., Age-specific prevalence of, and factors associated with, different types of urinary incontinence in community-dwelling Australian women assessed with a validated questionnaire. Maturitas, 2009. 62(2): p. 134-9.

93. Thuroff, J.W., et al., EAU guidelines on urinary incontinence. Eur Urol, 2011. 59(3): p. 387-400.

94. Altman, D., et al., Genetic influence on stress urinary incontinence and pelvic organ prolapse. Eur Urol, 2008. 54(4): p. 918-22. 


\section{Chapter 1}

95. Labrie, J., et al., Surgery versus physiotherapy for stress urinary incontinence. N Engl J Med, 2013. 369(12): p. 1124-33.

96. Siff, L.N., J.E. Jelovsek, and M.D. Barber, The effect of major depression on quality of life after surgery for stress urinary incontinence: a secondary analysis of the Trial of Midurethral Slings. Am J Obstet Gynecol, 2016.

97. Zorn, B.H., et al., Urinary incontinence and depression. J Urol, 1999. 162(1): p. 82-4.

98. Gratzke, C., et al., EAU Guidelines on the Assessment of Non-neurogenic Male Lower Urinary Tract Symptoms including Benign Prostatic Obstruction. Eur Urol, 2015. 67(6): p. 1099-109.

99. Barry, M.J., et al., The American Urological Association symptom index for benign prostatic hyperplasia. The Measurement Committee of the American Urological Association. J Urol, 1992. 148(5): p. 1549-57; discussion.

100. Oelke, M., et al., EAU guidelines on the treatment and follow-up of non-neurogenic male lower urinary tract symptoms including benign prostatic obstruction. Eur Urol, 2013. 64(1): p. 118-40.

101. van Koeveringe, G.A., et al., Detrusor underactivity: a plea for new approaches to a common bladder dysfunction. Neurourol Urodyn, 2011. 30(5): p. 723-8.

102. Van Koeveringe, G.A. and K.L. Rademakers, Factors impacting bladder underactivity and clinical implications. Minerva Urol Nefrol, 2015. 67(2): p. 139-48.

103. Suskind, A.M. and P.P. Smith, A new look at detrusor underactivity: impaired contractility versus afferent dysfunction. Curr Urol Rep, 2009. 10(5): p. 347-51.

104. Oelke, M., et al., Unravelling detrusor underactivity: Development of a bladder outlet resistance-bladder contractility nomogram for adult male patients with lower urinary tract symptoms. Neurourol Urodyn, 2015.

105. Luber, K.M., S. Boero, and J.Y. Choe, The demographics of pelvic floor disorders: current observations and future projections. Am J Obstet Gynecol, 2001. 184(7): p. 1496-501; discussion 1501-3.

106. Berghmans, B., et al., Prevalence and triage of first-contact complaints on pelvic floor dysfunctions in female patients at a Pelvic Care Centre. Neurourol Urodyn, 2016. Apr;35(4): p. 503-8.

107. Berghmans, B., et al., Prevalence and triage of first contact pelvic floor dysfunction complaints in male patients referred to a Pelvic Care Centre. Neurourol Urodyn, 2016 Apr;35 (4): p. 487-91

108. Davis, K.J., D. Kumar, and M.C. Wake, Pelvic floor dysfunction: a scoping study exploring current service provision in the UK, interprofessional collaboration and future management priorities. Int J Clin Pract, 2010. 64(12): p. 1661-70.

109. Diagnostic and Statistical Manual of Mental Disorders. 5 ed. 2013, Washington, DC: American Psychiatric Association.

110. Wang, P.S., et al., Twelve-month use of mental health services in the United States: results from the National Comorbidity Survey Replication. Arch Gen Psychiatry, 2005. 62(6): p. 629-40.

111. Ustun, T.B., et al., Global burden of depressive disorders in the year 2000. Br J Psychiatry, 2004. 184: p. 386-92.

112. Kessler, R.C., et al., Lifetime prevalence and age-of-onset distributions of DSM-IV disorders in the National Comorbidity Survey Replication. Arch Gen Psychiatry, 2005. 62(6): p. 593-602.

113. Demyttenaere, K., et al., Prevalence, severity, and unmet need for treatment of mental disorders in the World Health Organization World Mental Health Surveys. JAMA, 2004. 291(21): p. 2581-90.

114. Kroenke, K., Patients presenting with somatic complaints: epidemiology, psychiatric comorbidity and management. Int J Methods Psychiatr Res, 2003. 12(1): p. 34-43.

115. Cole, J.A., et al., Migraine, fibromyalgia, and depression among people with IBS: a prevalence study. BMC Gastroenterol, 2006. 6: p. 26.

116. Mykletun, A., et al., Prevalence of mood and anxiety disorder in self reported irritable bowel syndrome (IBS). An epidemiological population based study of women. BMC Gastroenterol, 2010. 10: p. 88.

117. Pinto-Sanchez, M.I., et al., Anxiety and Depression Increase in a Stepwise Manner in Parallel With Multiple FGIDs and Symptom Severity and Frequency. Am J Gastroenterol, 2015. 110(7): p. 1038-48. 
118. Leue, C., et al., Managing complex patients on a medical psychiatric unit: an observational study of university hospital costs associated with medical service use, length of stay, and psychiatric intervention. J Psychosom Res, 2010. 68(3): p. 295-302.

119. Akiskal, H.R.Z.e.a., Mood disorders., in Kaplan and Sadoxk's comprehensive textbook of psychiatry, S.V. Sadock B, Ruiz P., Editor. 2009. p. 1629-1838.

120. Evans, D.L., et al., Mood disorders in the medically ill: scientific review and recommendations. Biol Psychiatry, 2005. 58(3): p. 175-89.

121. Wittchen, H.U., Generalized anxiety disorder: prevalence, burden, and cost to society. Depress Anxiety, 2002. 16(4): p. 162-71.

122. Penninx, B.W., et al., Depression and cardiac mortality: results from a community-based longitudinal study. Arch Gen Psychiatry, 2001. 58(3): p. 221-7.

123. Roy-Byrne, P.P., et al., Anxiety disorders and comorbid medical illness. Gen Hosp Psychiatry, 2008. 30(3): p. 208-25.

124. Schildkraut, J.J., The catecholamine hypothesis of affective disorders: a review of supporting evidence. Am J Psychiatry, 1965. 122(5): p. 509-22.

125. Coppen, A., The biochemistry of affective disorders. Br J Psychiatry, 1967. 113(504): p. 1237-64.

126. Janowsky, D.S., et al., A cholinergic-adrenergic hypothesis of mania and depression. Lancet, 1972. 2(7778): p. 632-5.

127. Carroll, B.J., et al., A specific laboratory test for the diagnosis of melancholia. Standardization, validation, and clinical utility. Arch Gen Psychiatry, 1981. 38(1): p. 15-22.

128. Willner, P., J. Scheel-Kruger, and C. Belzung, The neurobiology of depression and antidepressant action. Neurosci Biobehav Rev, 2013. 37(10 Pt 1): p. 2331-71.

129. Goodwin FK, J.K., Manic-Depression illness. 2nd ed. 2007, Nwe York: Oxford University Press.

130. Belzung, C., P. Willner, and P. Philippot, Depression: from psychopathology to pathophysiology. Curr Opin Neurobiol, 2015. 30: p. 24-30.

131. Price, J.L. and W.C. Drevets, Neural circuits underlying the pathophysiology of mood disorders. Trends Cogn Sci, 2012. 16(1): p. 61-71.

132. Lang, P.J., M.M. Bradley, and B.N. Cuthbert, Emotion, motivation, and anxiety: brain mechanisms and psychophysiology. Biol Psychiatry, 1998. 44(12): p. 1248-63.

133. Duval, E.R., A. Javanbakht, and I. Liberzon, Neural circuits in anxiety and stress disorders: a focused review. Ther Clin Risk Manag, 2015. 11: p. 115-26.

134. Baur, V., et al., Evidence of frontotemporal structural hypoconnectivity in social anxiety disorder: A quantitative fiber tractography study. Hum Brain Mapp, 2013. 34(2): p. 437-46.

135. Zigmond, A.S. and R.P. Snaith, The hospital anxiety and depression scale. Acta Psychiatr Scand, 1983. 67(6): p. 361-70.

136. Bierhoff, F., Irritable bladder in the female. Am J Med Sci, 1900. 120: p. 670.

137. Straub, L.R., H.S. Ripley, and S. Wolf, Disturbances of bladder function associated with emotional states. J Am Med Assoc, 1949. 141(16): p. 1139-43.

138. Huppertz, B.J., [Psychosomatic aspects of irritable bladder. A review]. Urologe A, 1986. 25(2): p. 84-9.

139. Engel, W.J., Uropsychiatry. J Mich State Med Soc, 1964. 63: p. 273-7.

140. Cortes, E., et al., The psychology of LUTS: ICI-RS 2011. Neurourol Urodyn, 2012. 31(3): p. 340-3. 

Chapter

\section{Affective symptoms and the overactive bladder - a systematic review}

Vrijens DMJ*, Drossaerts JM*, van Koeveringe GA, Van Kerrebroeck Ph, van Os J, Leue $C$.

Journal of Psychosomatic Research 2015;78:95-108

${ }^{*}$ Both authors contributed equally 


\section{ABSTRACT}

\section{Background}

Overactive bladder syndrome (OAB) is characterised by urgency symptoms, with or without urgency incontinence, usually with frequency and nocturia. Although literature suggests an association between $\mathrm{OAB}$, depression and anxiety; no systematic review has been presented.

\section{Objective}

Systematically review the literature on the association of affective conditions with OAB.

\section{Methods}

Systematic review according to the PRISMA guidelines. This review is registered in the PROSPERO prospective register of systematic reviews (CRD4201400664).

\section{Results}

Forty-three articles were included, describing more than 80.000 subjects. Depression and $O A B$ were positively associated in 26 studies, anxiety and $O A B$ in 6 studies. Longitudinal studies reported: a) OAB subjects who developed depression/anxiety or b) depressed/anxious subjects developing $O A B$, or c) both. Quality of evidence in studies reporting an association between the co-occurrence of $O A B$ and depression, was rated level 3 conform the GRADE framework. Evidence reporting on the co-occurrence of anxiety and $O A B$ was rated GRADE level 2. Longitudinal associations between new onset of $O A B$ in depressive subjects was GRADE level 2. Evidence reporting association of OAB with anxiety in longitudinal studies was of GRADE level 1.

\section{Conclusion}

To our knowledge, this systematic review is the first to give a comprehensive qualitative overview on the association between $O A B$ and affective symptoms. Many evaluated studies failed to note longitudinal changes and lacked evidence of causality. Still, results revealed an association between $\mathrm{OAB}$ and affective symptoms and there is evidence for new onset of $O A B$ in depressive subjects, but further research is necessary to examine the strength of the effect. 


\section{INTRODUCTION}

Depression and anxiety are classified as affective disorders according to the DSM-IV psychiatric diagnostic criteria ${ }^{1}$. According to a survey of the World Health Organisation, anxiety is globally the most common psychiatric disorder ${ }^{2}$. The lifetime prevalence of anxiety disorders in the US adult population is around $18 \%^{2}$. Depression has a high lifetime prevalence as well, ranging from 2 to $15 \%{ }^{3}$.

Overactive bladder (OAB) is defined by the International Continence Society (ICS) as a symptom complex of urgency, usually accompanied by frequency (voiding 8 or more times in a 24 hour period) and nocturia (awakening at night to void), with (OAB wet) or without (OAB dry) urgency urinary incontinence (UUI) ${ }^{4}$. Approximately $16-17 \%$ of the adult population is affected by $O A B$ : $43 \%$ of adult women experience $O A B$ symptoms 'sometimes' and 33\% 'often' 5, 6 . The health care costs of $O A B$ are high and around 12 billion USD per year"

Although high prevalence rate would suspect the opposite, OAB is often underdiagnosed and subsequently undertreated, mainly because of patients' reluctance to seek medical help ${ }^{8}$. Only $27 \%$ of people with $O A B$ were receiving treatment in a large population based survey ${ }^{6}$. One possible reason for not seeking help in OAB may be caused by stigma perception ${ }^{8}$. Another is that people may assume that $\mathrm{UI}$ is part of the normal aging process ${ }^{9}$. In addition, an inverse relationship between depressive symptoms and healthcare seeking in patients with $O A B$ is reported ${ }^{10}$.

A relationship between common affective disorders (e.g. depression and anxiety disorders) and lower urinary tract symptoms (LUTS) has been described as early as 1964 ${ }^{11}$. The importance of mental disorders in the aetiology of urgency incontinence was again emphasized more recently ${ }^{12,13}$. In 2011 the International Consultation on Incontinence-Research Society (ICl-RS) organized a think tank on psychological factors and LUTS $^{14}$. It was noted that not only OAB but also other LUTS may be associated with affective conditions, concluding that 'the possibility of causation and or maintenance of lower urinary tract symptoms through psychological causes needs further research ${ }^{14}$. At present there has been renewed interest in the matter, reflected by a quadrupling in publications found through PubMed/EMBASE search on the topic since 2000 (searched for anxiety, depression and lower urinary tract symptoms before and after 2000).

Despite this growing interest, the exact nature of the association between bladder symptoms and mental conditions, specifically affective disorders, remains unknown. A systematic review using strict definitions may inform the field regarding the strength of the associations between affective disorders and $\mathrm{OAB}$ and may possibly enhance insight in underlying pathophysiological mechanisms and treatment recommendations at the "bladder-brain-axis".

The aim of the current study was to (i) review articles providing evidence on the association of affective conditions with $\mathrm{OAB}$; (ii) to examine the direction and strength of the 
association; (iii) to gauge the possible influence of method and design factors in terms of occasioning bias or confounding; (iv) to formulate considerations for future research.

\section{METHODS}

This systematic review was registered in PROSPERO under number CRD42014006641 ${ }^{15}$.

\section{Inclusion and exclusion criteria}

A literature search for full length original articles meeting the following inclusion criteria was carried out: a) adults; b) patients with overactive bladder, urinary urgency incontinence (and/or frequency or nocturia); not initiated by surgery, using a questionnaire, voiding diaries or urodynamic evaluation to state symptoms; c) assessment of depressive symptoms and/or anxiety using a validated multi-item scale or a structured diagnostic interview; d) reporting an association between urinary symptoms and depression or anxiety; f) published in English in a peer-reviewed journal. Reviews, expert opinions, abstracts and comments were excluded.

\section{Literature search}

PRISMA guidelines were used concerning preferred reporting items for systematic reviews ${ }^{16}$. The following databases were searched: Medline (PubMed), Embase, Web of Knowledge, Cochrane and PsycINFO. There was no age restriction on the searched articles, and the last search was dated December $31^{\text {st }}$ 2013. The detailed query is listed in the appendix (table C.1)

These database searches were supplemented by hand searching the reference lists of eligible articles. Two authors (DV and CL) independently examined titles, abstracts and full-text articles. Name of authors, institution and journal of publication were not blinded. One author was a content expert on overactive bladder (DV), the other an expert in the field of affective disorders in somatic conditions (CL). Consensus was obtained in $85 \%$ of the manuscripts; the remaining $15 \%$ of disagreements were handled by discussion. 1249 abstracts were identified in the database searches, and after removal of duplicates, 925 remained for screening on relevance. In the reference lists an additional 54 abstract were found. Eventually, 117 full text articles were assessed for eligibility, 74 articles were excluded resulting in a final set of 43 articles (out of 37 studies), which were included in this review. Errata were examined if articles were retracted since publication; there were none. Attempts were made to obtain unpublished results to diminish effects of reporting bias. The flow-chart of the selection process is depicted in Figure 1. 


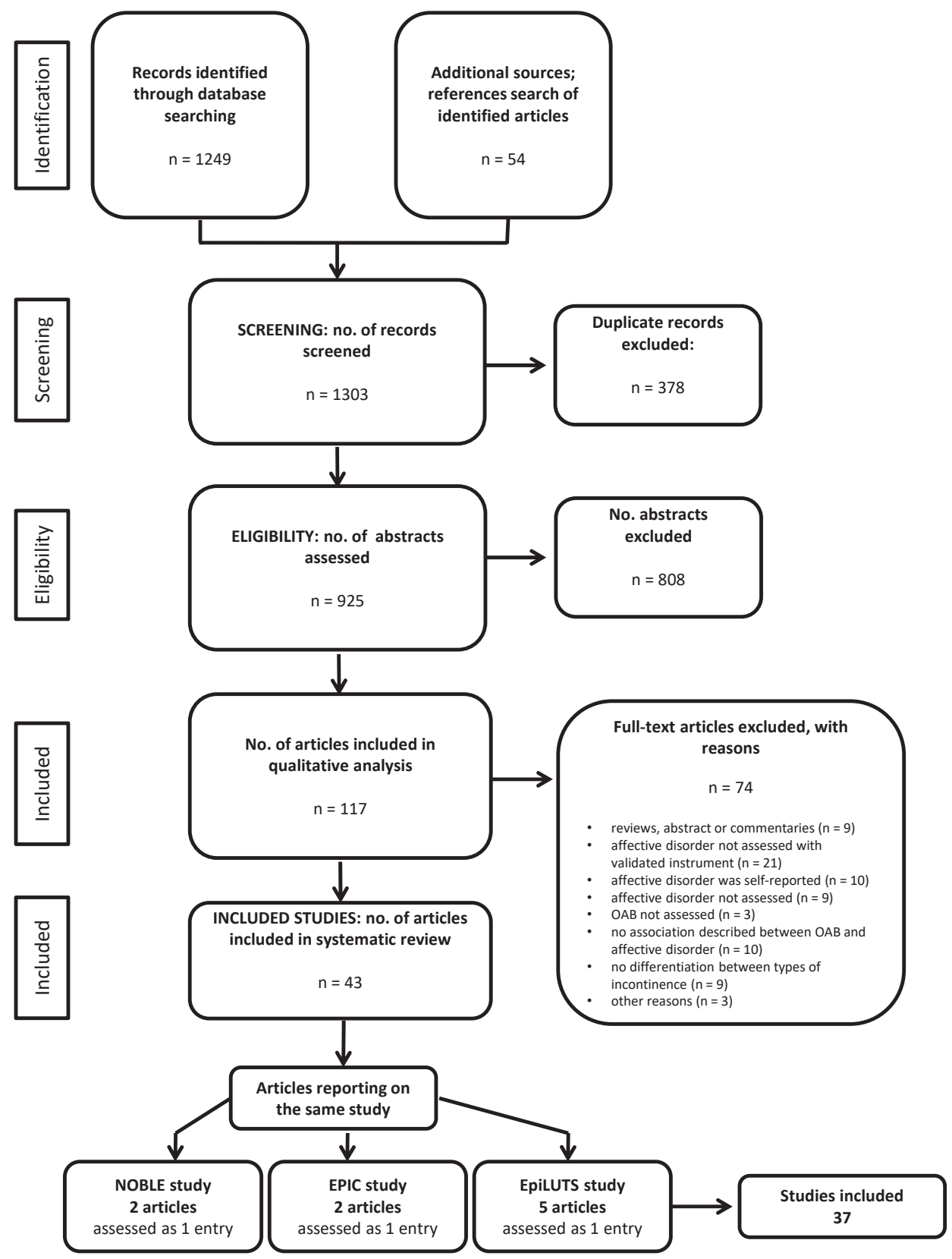

Figure 1. Flow-chart of selection process, according to the PRISMA guidelines, $2009^{16}$. 


\section{Data extraction and quality assessment}

Two authors performed data extraction and quality assessment independently (DV, urologist and JD, PhD student), using a standard data extraction form. The data extraction form was pilot tested and modified accordingly before further use. Consensus was obtained in $90 \%$ of the articles; the remaining $10 \%$ of disagreements were solved by discussion. Multiple reports on the same study were collated as one entry of interest. Level of evidence of each article was stated according to the University of Oxfords' Centre for Evidence-based Medicine (CEBM) documents ${ }^{17}$. Assessment of risk of bias was performed by using the Cochrane Collaboration's tool for assessing risk of bias ${ }^{18}$. Six domains of bias were taken into account: selection bias, performance bias, detection bias, attrition bias, reporting bias and other bias. The assessment was finished by applying the 'Grading of Recommendations, Assessment, Development, and Evaluation' (GRADE) framework to rate overall quality of evidence for each reported outcome measurement ${ }^{19}$.

\section{RESULTS}

Forty-three articles regarding 37 studies were included in the review. Thirty-two articles were about depression, 9 about anxiety (and depression) and two about anxiety only. Diagnosing $O A B$ was carried out according to the ICS definition of OAB in 33 articles. In 10 articles, the ICS definition had not been used. Three of these articles were published before establishment of the ICS definition in $2002^{20-22}$. Retrospectively these 10 articles adhered to the ICS definition and were included. Twelve studies used additional instruments, such as voiding diaries or urodynamic studies. A validated measurement of depressive and/or anxiety symptoms was used in all studies (Table A.1). Most articles described cross-sectional $(n=29)$ study designs. The remainder were cohort studies $(n=10)$ and randomised controlled trials $(n=4)$ (Table 1$)$. There was a wide range in mean age (between 29 and 89 years old) and the number of people included per study (35 to 30.000). Overall a little more than 80.000 subjects were included. Most included subjects were women, but for approximately 30.000 men, gender-specific analysis was reported. The eligibility of performing a meta-analysis was examined. Due to the heterogeneity of the included studies and the small number of longitudinal studies, it was not feasible to perform a meaningful meta-analysis. Hence a qualitative synthesis was undertaken.

Overall in most articles all risk of bias domains were assessed as low risk or unclear risk. Also some domains were not applicable for several articles (inherent to study design) (Table 2). "Other bias" was around $47 \%$ across articles, such as ascertainment bias 23, 24 and sick quitter bias ${ }^{25-27}$. 
A positive association between depression and $O A B$ was found in the majority of studies $(n=26)$ (Table 3). There was no association of OAB with depression in 9 studies (Table 3). Two studies reported about anxiety and OAB only (not depression); both revealed a positive association. In the longitudinal studies, associations in different directions were present: $O A B$ subjects developing depression/anxiety and/or depressed/anxious people developing $\mathrm{OAB}$, or both (all compared with control subjects).

\section{Depressive symptoms and OAB: cross-sectional studies}

Of the 29 cross-sectional studies, nine studies did not show an association of OAB with affective conditions $20,22-24,28-32$. The others revealed positive associations. No studies reported a negative association. Nine articles reported results from the same studies, respectively the NOBLE, EPIC and EpiLUTS studies (Table 1). The National Overactive Bladder Evaluation (NOBLE) study was conducted to establish estimates of the prevalence of $O A B$ and of the individual burden of illness; the sample was 5204 US adults. Women suffering from $O A B$, with or without UUI, had significantly worse scores on the Centre of Epidemiologic Studies Depression Scale (CES-D), than OAB-negative controls ${ }^{33}$. Another report did not find an association between $O A B$ and depression ${ }^{34}$. The Epidemiology Urinary Incontinence and Comorbidities (EPIC) study was a cross-sectional survey in five countries representing 19.165 subjects. The two EPIC articles reported significantly more cases with depression in OAB, with or without UUI, compared to controls $^{35,36}$. The Epidemiology of Lower Urinary Tract Symptoms (EpiLUTS) study was a cross-sectional survey conducted in the USA, UK and Sweden, representing 30.000 subjects ${ }^{10,26,37-39}$. Subjects with OAB reported the highest rate of anxiety (31\%) and depressive symptoms $(27 \%)^{37}$.

The presence of major depression led to a significant increase in a patient's urinary incontinence symptom reporting ${ }^{40}$. A Japanese study of 833 elderly revealed a significant association between $\mathrm{OAB}$, and depressive symptoms based on the Geriatric Depression Scale (GDS-30) ${ }^{25}$. Multivariate analysis showed that the risk of having OAB was significantly higher in subjects with depressive symptoms. In contrast, in incontinent obese women there was no association between depressive symptoms with overall incontinent episodes ${ }^{41}$.

However, there was an association of depressive complaints with the number of stress incontinence episodes ${ }^{41}$. Another cross-sectional study revealed the strongest association between incontinence and depression for mixed urinary incontinence (MUI) and $\mathrm{UUI}^{42}$. Ito et al. compared people with depression diagnosed at the psychiatric clinic, with age-matched individuals without depression. There was significantly more urinary urgency and UUI in the medicated depressed women compared to controls ${ }^{43}$. A large Swedish twin study representing 14098 women aged 20-46 showed that major depression assessed by Composite International Diagnostic Interview-Short Form (CIDISF) and depressive symptoms by CES-D were positively associated with all subtypes of 
$\mathrm{UI}^{44}$. In contrast, Chiara et al. did not find any difference in subjects with OAB concerning depressive mood or anxiety ${ }^{20}$, but found anger, irritability and general hypochondria being more prevalent in subjects with OAB than in subjects with SUI (Table 1).

\section{Depressive symptoms and OAB: longitudinal studies}

Thirteen longitudinal studies were included, of which 9 were cohort studies and 4 were randomised controlled trials. Overall, almost 40.000 subjects were analysed in these studies. Regarding affective complaints, the data of two longitudinal studies were only cross-sectional analysed and focuses upon the presence of depressive symptoms and OAB at baseline ${ }^{41,45}$.

Most longitudinal studies $(n=9)$ revealed a positive association of OAB with depressive symptoms, whereas the remaining four did not find any association ${ }^{23,30-32}$. Several studies $(n=4)$ showed a positive association of OAB onset in persons with depressive symptoms ${ }^{27,30,46,47}$. The studies of Perry et al. and van de Pol et al. revealed a positive association of onset of depressive symptoms in persons with $O A B{ }^{27,30}$. One study revealed a positive association of onset of anxiety symptoms in persons with $O A B$ and reported a positive association between the onset of $O A B$ in anxious patients ${ }^{27}$. $A$ population based longitudinal study with six years of follow up revealed that depressive mood was a sign predictor of incident urgency related $\mathrm{UI}{ }^{46}$. A large prospective study of people older than 65 years demonstrated that depression measured by GDS was an independent risk factor for new onset $O A B$ one year later ${ }^{47}$. The aim of another important prospective longitudinal study was to investigate the association between anxiety and depression and urgency incontinence in women ${ }^{27}$. Incident cases of anxiety and depression were predicted by the presence of UUI at baseline. Incident cases of UUI were predicted by anxiety, but not by depression, at baseline. Anxiety, UUI and frequency appeared to interact and exacerbate each other ${ }^{27}$. At 36 weeks gestation OAB was significantly associated with depression in multivariate analysis, but there was no association one year after childbirth ${ }^{30}$. Similar, no association was found between depression and urinary incontinence in women followed six years through menopausal transition $^{31}$.

\section{Randomised controlled trials}

Among the longitudinal studies there were four randomised controlled trials which showed that successful treatment of OAB led to less affective symptoms regardless of the investigated intervention ${ }^{23,48-50}$. Furthermore, one study showed only significantly less anxiety ${ }^{23}$. A cohort study without a control arm in solely men, revealed that mean $\mathrm{BDI}$ and bladder symptoms improved significantly after 6 months of treatment with oxybutynin. The proportion of men with BDI scores indicating depression $(>12)$ decreased from 23.9 to $17.9 \% .{ }^{51}$. The randomised clinical trials consisted of comparing interventions ${ }^{49,50}$, interventions with placebo ${ }^{23}$ or timing of intervention ${ }^{48}$. OAB symp- 
toms improved significantly more in the medication group after 12 weeks of treatment in a double blind placebo controlled trial of tolterodin in sexually active women. Baseline HADS scores were indicative for anxiety, but not for depression. There was a significant decrease of the mean HADS-A score, from aberrant to normal, between the two groups ${ }^{23}$. Ozededeli et al. compared effects of trospium hydrochloride and electrical stimulation during a 6 week follow up in two groups of women with OAB on urodynamic parameters, bladder diaries and BDI. There was significant improvement on all parameters in both groups ${ }^{49}$. Similar results were found by a secondary analysis of an assessorblinded randomised controlled trial comparing three forms of physiotherapy with medication for self-reported UUI ${ }^{50}$. Although clinically diagnosed depression was an exclusion criterion, 36 (22\%) women had a CES-D score $>16$ at baseline. These women had a significant decrease in CES-D scores 3 and 12 months after therapy, which was accompanied by less $O A B$ related symptoms.

At the baseline of a study population with $O A B, 73 \%$ of the subjects had some level of depression. After randomisation between early and late implantation of a sacral neuromodulator only in the early implant group the BDI scores declined significantly ${ }^{48}$ (Table 1).

\section{Anxiety and $O A B$ studies}

Thirteen articles concerning nine studies reported about anxiety. Six studies revealed a positive association (Table 3). Three studies did not show any association ${ }^{20,22,52}$. The two case-control studies yielded contradictory conclusions ${ }^{22,53}$. Most cross-sectional studies showed a positive association between urological symptoms and anxiety. In a crosssectional population-based study amongst 5321 young women UI was associated with both anxiety and depression. The strongest associations were found for MUI and UUI ${ }^{42}$.

American, English and Swedish EpiLUTS data, including 30.000 men and women, showed that $O A B$ symptoms were significantly associated with positive HADS anxiety sub-scores in men and women ${ }^{38}$. In the US, EpiLUTS' respondents who experienced bothersome $\mathrm{OAB}$ symptoms, were more likely to report anxiety than those with $\mathrm{OAB}$ without bothersome symptoms ${ }^{39}$. Data from the UK and Sweden yielded similar results. Increasing levels of anxiety were strongly predictive of $O A B$ bother in both men and women ${ }^{10}$. From a longitudinal perspective, a positive association between anxiety and urgency incontinence was reported in a prospective postal survey of women with follow up one year later. Anxiety measured by HADS was present in $56.6 \%$ of those with UUI. Anxiety was not only associated with UI, but also with a number of other urinary symptoms. The presence of UI and/or frequency (but not SI or urgency) predicted incident cases of anxiety one year later. Vice versa, the presence of anxiety at baseline predicted incident cases of UI one year later. Anxiety, UUI and frequent micturition, appeared to interact by exacerbating each other ${ }^{10}$ (Table 3 ). 
Chapter 2

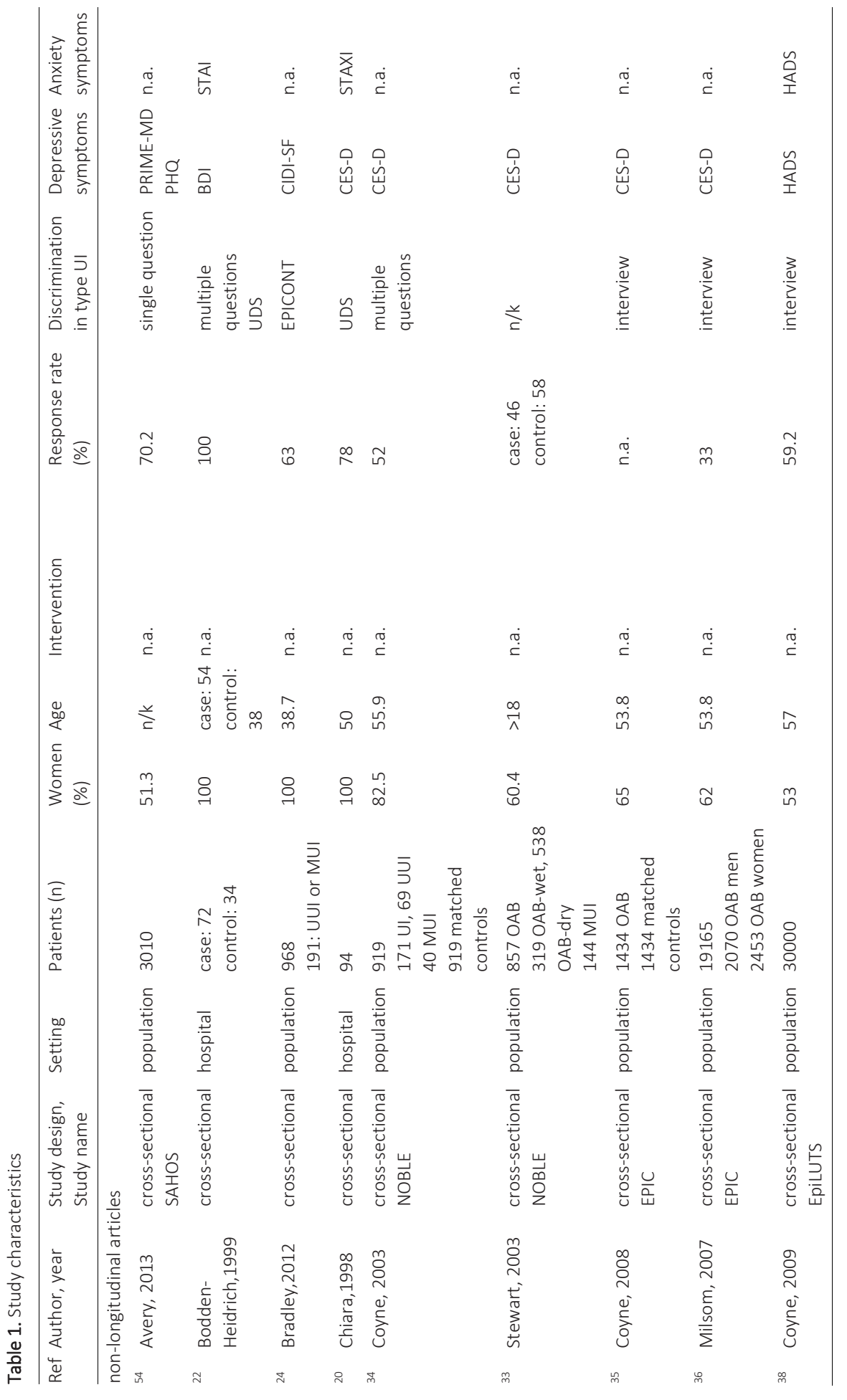




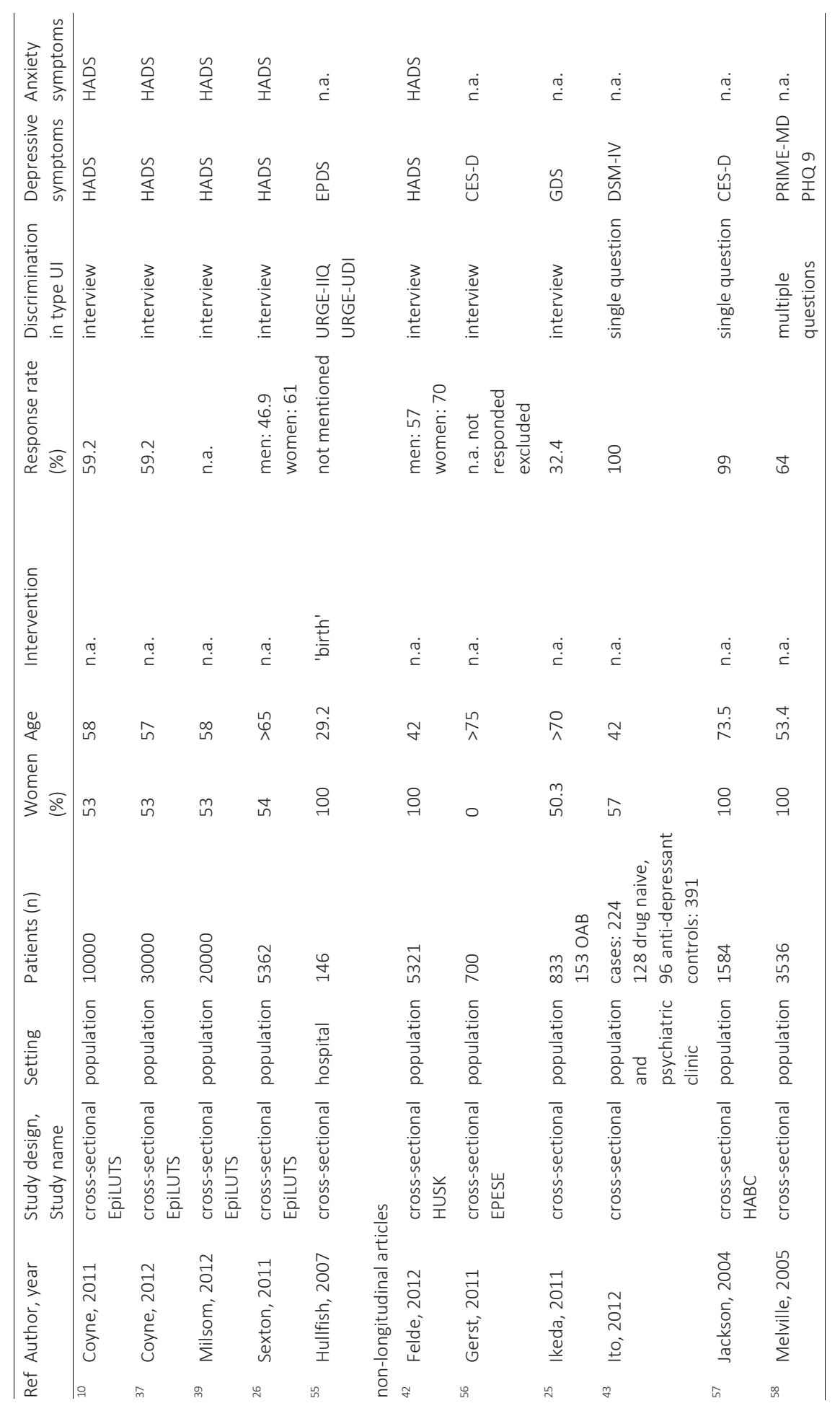




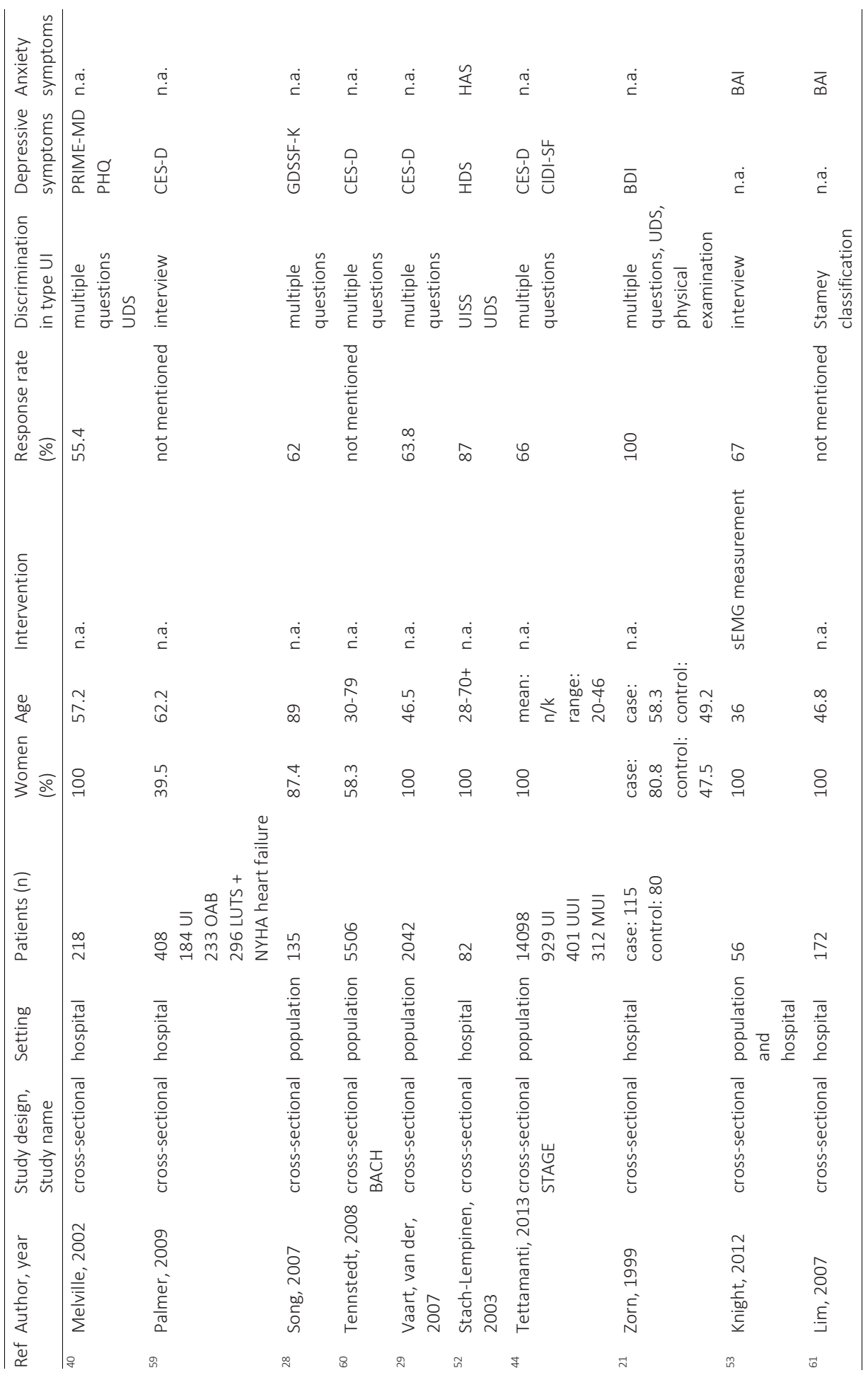




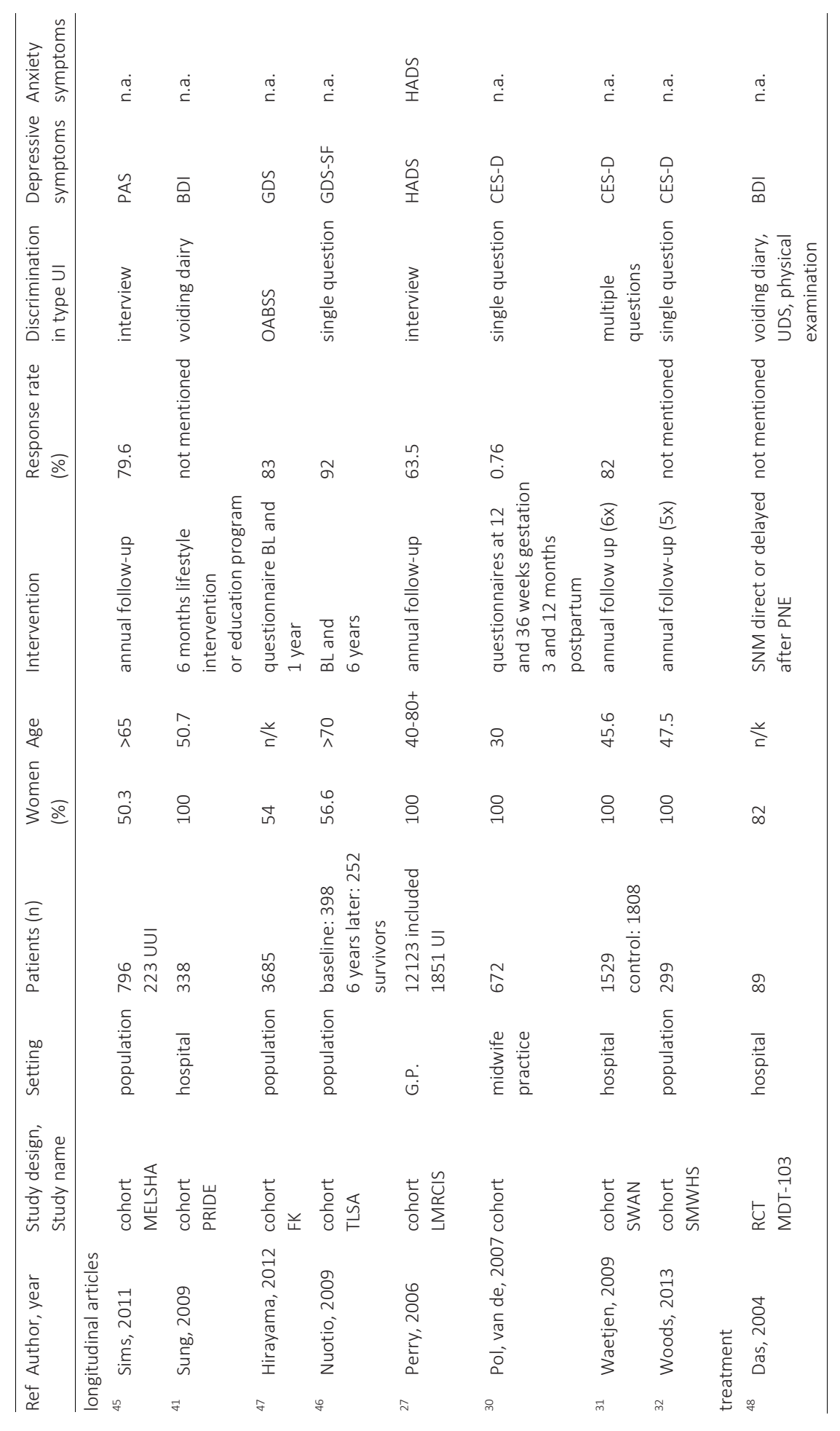




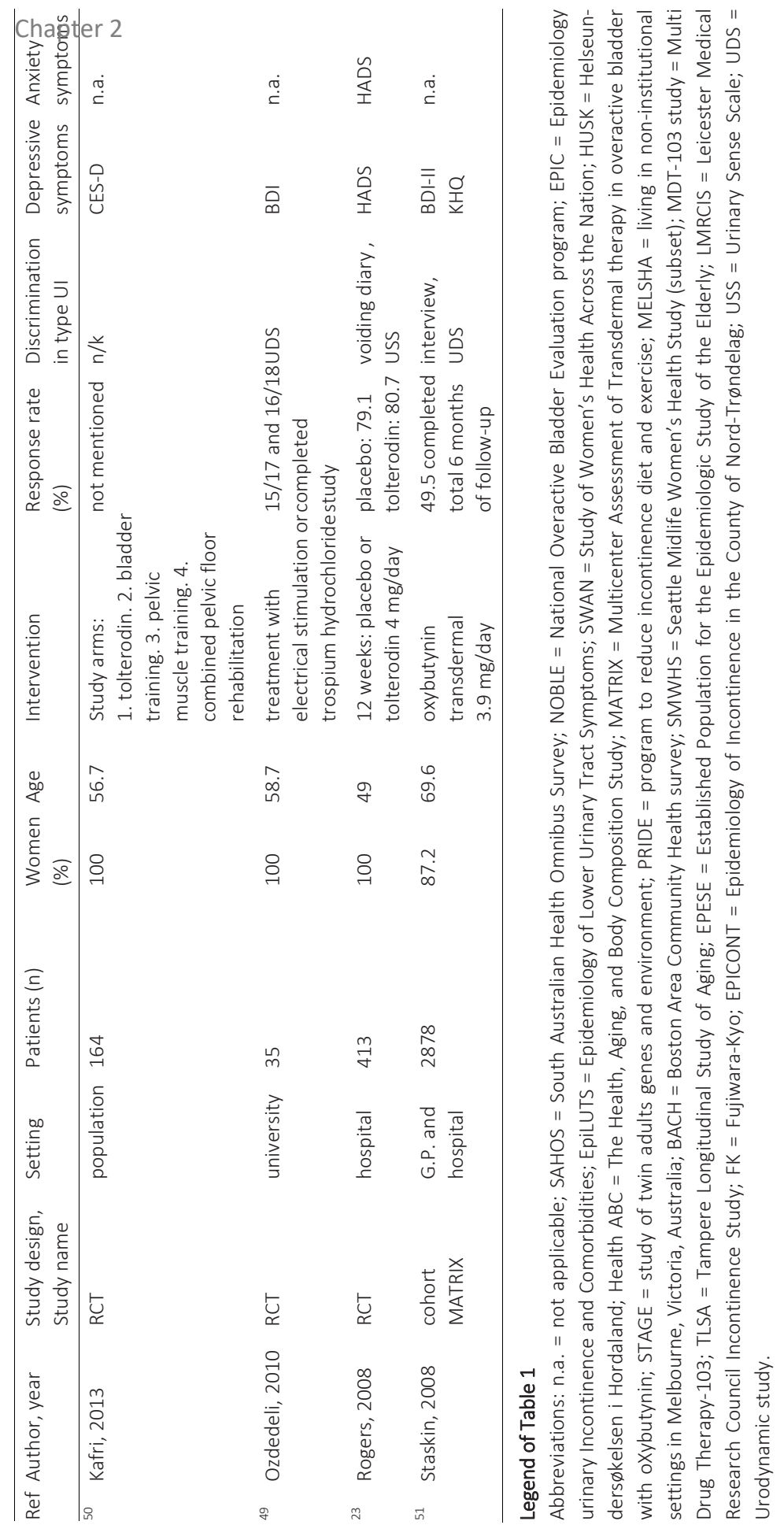


Twenty-two articles included men, Table 1. Of these, ten studies reported specifically about men (NOBLE, EPIC, EpiLUTS). Eight studies concluded that there is an association of $O A B$ with depressive or anxiety symptoms. The remaining two studies including men and women reported an association of $O A B$ with anxiety and depression for women, but not for men ${ }^{45,48}$.

Five articles from the EpiLUTS study included $47 \%$ men. Men with storage symptoms reported lower rates of depression (15.4\% vs. $26.6 \%$ ) and anxiety (12\% vs. $14.9 \%$ ) than women ${ }^{38}$. Men and women with bothersome OAB symptoms reported the highest rate of anxiety and depressive complaints ${ }^{10,39}$. In the UK, this was higher in men than in women ${ }^{10}$ and increased anxiety was predictive of OAB bother ${ }^{10}$. Depressive symptoms predicted treatment seeking in men but not in women ${ }^{10,39}$. In an older subset of EpiLUTS, anxiety $(16.4 \%$ vs. $3.3 \%, p<0.001))$ and depressive symptoms $(17.7 \%$ vs. $4.0 \%$, $\mathrm{p}<0.001$ ) were more frequent in men with $\mathrm{OAB}$ versus men without $\mathrm{OAB}$. Furthermore, the rate of urgency (25.9\%) was higher than in aged-matched controls ( $9 \%, p \leq 0.01)$ in depressed men without anti-depressants, recruited from the psychiatric clinic ${ }^{43}$. Hirayama et al. reported results of a questionnaire based follow up in 1410 men ${ }^{47}$. Depression and the number of men was significantly more frequent in the new onset $O A B$ group. Both parameters were independent risk factors for $O A B$ in multivariate analyses ${ }^{62}$. Moreover, men with OAB-wet had significantly poorer scores on the CES-D scale compared to men who had OAB without incontinence ${ }^{33}$.

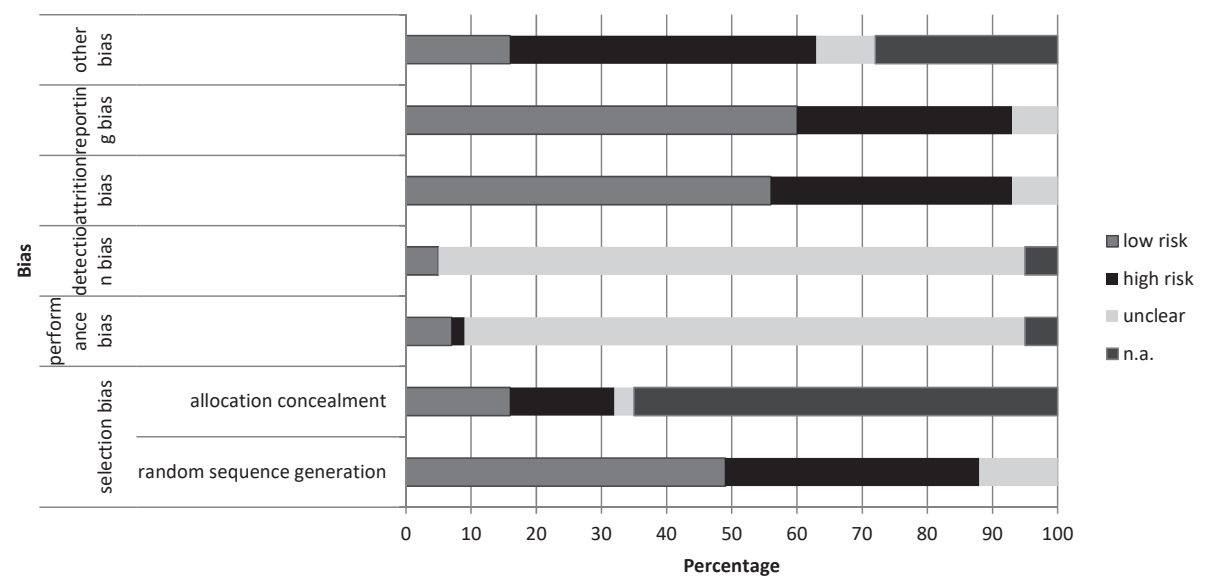

Table 2. Assessment of risk of bias (Cochrane); summarized. Comprehensive information per article is depicted in table B.1.

Abbreviations: n.a. = not applicable. Risk of bias is summarized. Each criterion was assessed by a questionbased entry, with the judgment being 'yes' indicating 'low' risk of bias, 'no' indicating a 'high' risk of bias, and 'unclear'. Risk of bias for each study was summarized as: 'low' risk of bias, when a low risk of bias was described for all key domains; 'unclear' risk of bias, when the bias was deemed to be unclear in one or more of the domains; and 'high' risk of bias, when one or more domains were judged to be of a high risk of bias. 


\section{QUALITY ASSESSMENT OF THE INCLUDED LITERATURE}

Level of evidence was stated per article as defined in publications of the University of Oxfords' Centre for Evidence-based Medicine (CEBM) ${ }^{17}$ (Table B.1 and summarised in Table 3). Risk of bias assessment was performed according to the Cochrane Collaboration guidelines (Table 3). The GRADE approach ${ }^{19}$ was applied to rate the quality of evidence for each outcome measure (Table D.1). An outcome-level assessment of risk of bias was maintained, after assessing the risk of bias for the studies as a whole, as the quality of evidence may differ across outcomes within one study.

The GRADE level of the outcome 'co-occurrence OAB and depressive symptoms' was upgraded (+1), because of the very large group of subjects. In male subjects this cooccurrence was downgraded (-1), because of evident selection bias (dismissing existing prostate (obstruction) problems). Hirayama et al. did not report about new onset of depressive outcomes, additional inquiry revealed that only 61 (7\%) of 834 OAB patients developed depression, compared to $5 \%$ developing depressive symptoms in the control $\operatorname{group}^{47}$.

Adhering to the GRADE approach, quality of evidence concerning co-occurrence of $O A B$ and depression was rated GRADE level 3. Evidence reporting an association the cooccurrence of anxiety and OAB was GRADE level 2. Longitudinal associations between new onset of $O A B$ in depressive subjects was GRADE level 2. Evidence reporting $O A B$ in longitudinal association with anxiety was GRADE level 1. 


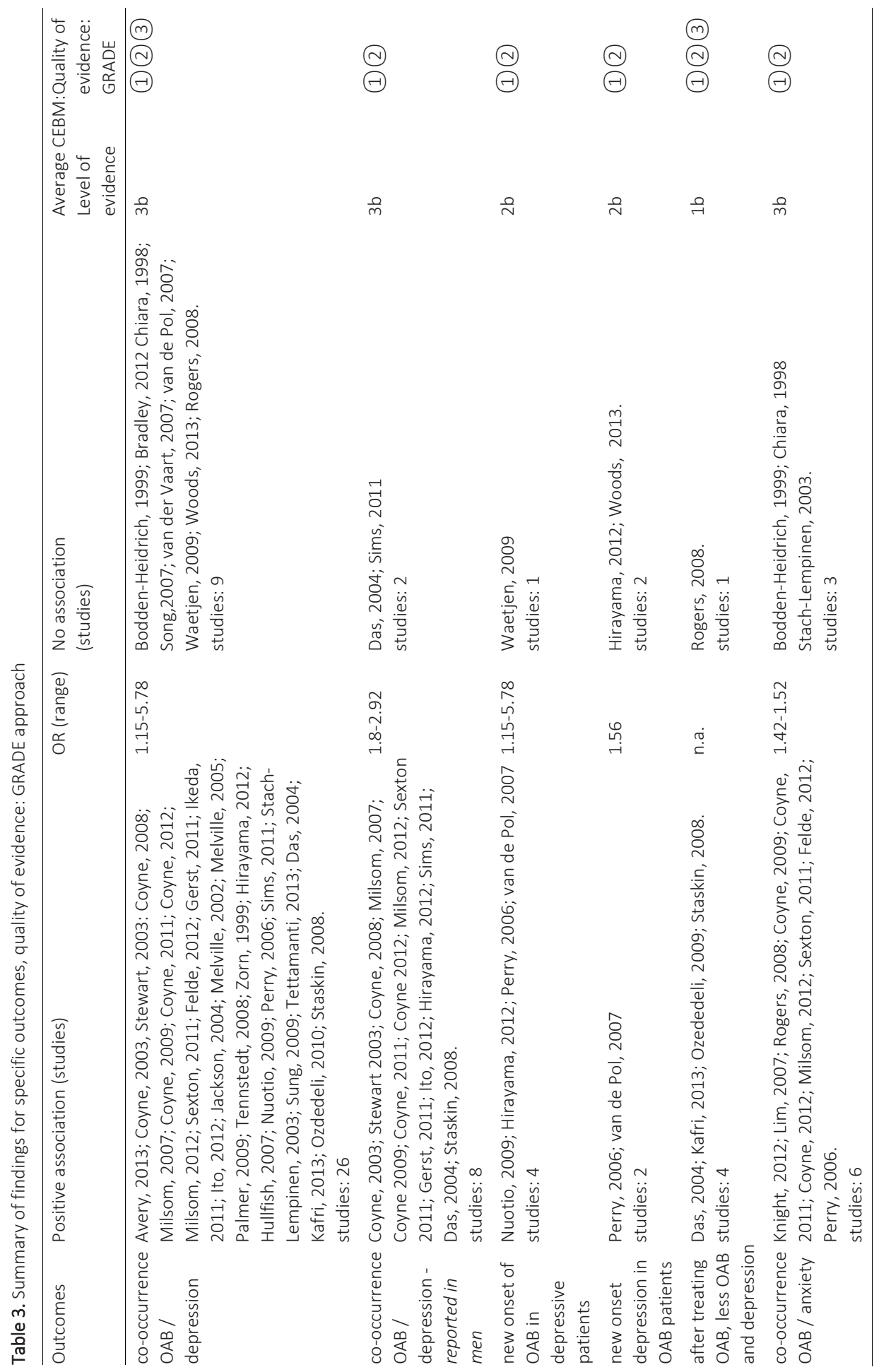


Chapter 2

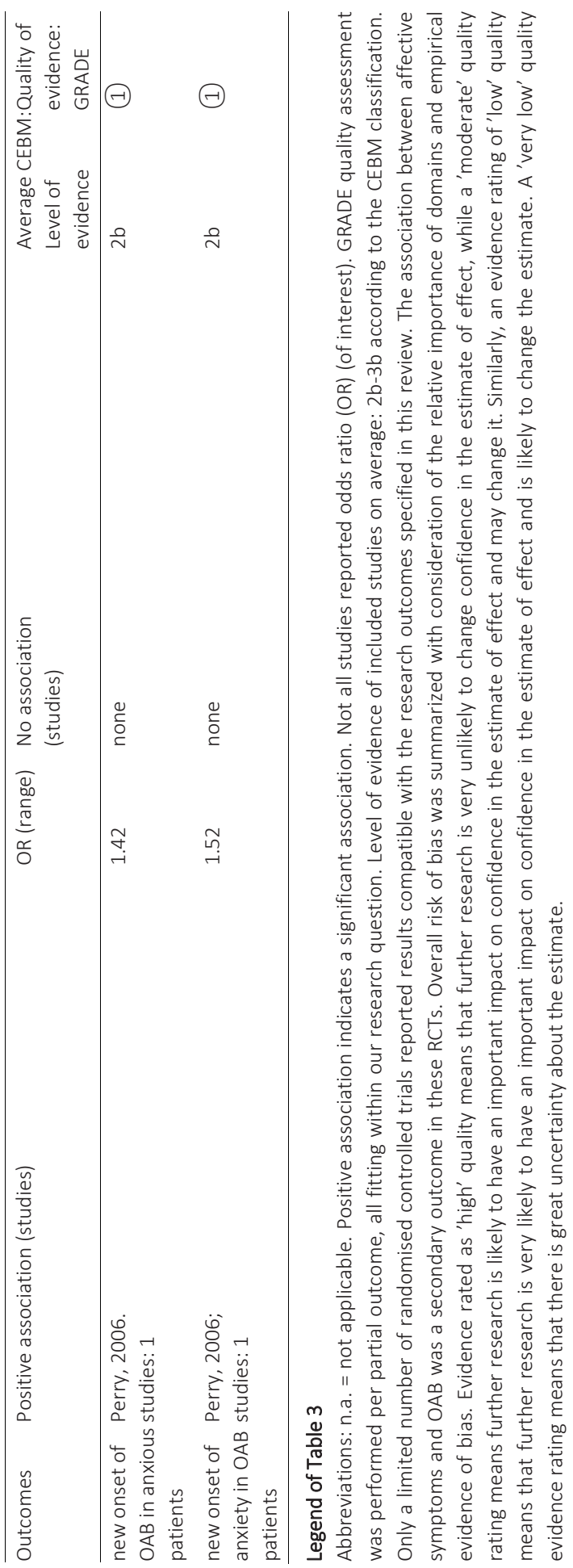




\section{DISCUSSION}

To our knowledge, this systematic review is the first to give an extensive qualitative overview of the current literature on the association between OAB and affective symptoms. The aim of the current study was to (i) review articles providing evidence on the association of affective conditions with $\mathrm{OAB}$; (ii) to examine the direction and strength of the association; (iii) to gauge the possible influence of method and design factors in terms of occasioning bias or confounding; (iv) to formulate considerations for future research.

Forty-three articles reporting 37 studies were included, comprising more than 80.000 subjects in total. Of these articles 32 concern depression, 2 are solely about anxiety and 9 concern both. Regarding depression and overactive bladder there is a positive association in 26 studies. Regarding anxiety six studies reveal a positive association. In longitudinal studies, different directional associations were reported: a) OAB subjects who develop depression/anxiety or b) depressed/anxious subjects who develop $O A B$, or $c$ ) both. The quality of the evidence in studies reporting an association between the co-occurrence of $O A B$ and depression, was rated level 3 conform the GRADE framework. Evidence reporting an association the co-occurrence of anxiety and $O A B$ was GRADE level 2. Longitudinal associations between new onset of OAB in depressive subjects was GRADE level 2. Evidence reporting an association of OAB in longitudinal association with anxiety was GRADE level 1.

No RCT was found with exact outcome measures as stated in this systematic review. Nevertheless, 4 RCTs were included, because they reported outcome measures of interest. These trials reveal a positive association between $O A B$ and affective symptoms because successful treatment of $O A B$ resulted in a significant decrease of affective complaints.

The main findings of this systematic review are that there is abundant and quality evidence of a positive association between co-occurrence of $O A B$ and depression and to a lesser extent the same accounts for an association between co-occurrence of OAB and anxiety. However, many evaluated studies failed to note longitudinal changes and lacked evidence of causality. The association between $O A B$ and depressive symptoms could to be bidirectional in nature. Only strong evidence is found concerning the new onset of OAB in depressive patients (GRADE level 2). Evidence of the uni- or bidirectional association between $O A B$ and anxiety was too poor to make meaningful statements (GRADE level 1).

Furthermore it is hard to identify the role of urgency incontinence in $O A B$, because only a few articles reported about separate results for $O A B$ dry and wet. There was increased rate of depression independent of continence status ${ }^{33,36}$ and higher CES-D scores in $\mathrm{OAB}$ wet were reported ${ }^{33,35}$. On the other hand, Ikeda et al. found an association between OAB-dry (without incontinence) and depression ${ }^{25}$. 


\section{Possible explanations for association}

One possible explanation for the association of affective disorders with OAB may be that UI can lead to social and functional impairment resulting in accumulation of stress. This, in turn, may lead to depression ${ }^{58}$. The association between affective disorders and $O A B$ may further be explained by the fact that both syndromes share common biological pathways. For instance, serotonin and norepinephrine, as well as the interactions between the two neurotransmitter systems are thought to play a role in the aetiology of depression ${ }^{47}$. Serotonin depletion is thought to contribute to depression and selective serotonin reuptake inhibitors (SSRIs) are effective in depression treatment ${ }^{63}$. Moreover, in anxiety disorders, it is likely that the serotonergic system plays a role in regulation of anxiety both during development and in adulthood ${ }^{64}$. Additionally, serotonin plays a role in bladder function. Several animal studies revealed that lowering serotonin levels in the central nervous system (CNS) was accompanied by urinary frequency and detrusor overactivity ${ }^{65}$. Most experimental studies in rats and cats indicated that activation of the central serotonergic system depresses reflex bladder contractions and increases the bladder volume threshold for micturition induction ${ }^{66}$. Affective disorders may thus be linked to $O A B$, due to altered serotonin function. Moreover a rat model of depression caused by lowering of serotonin in the CNS, produced OAB manifestation (urinary frequency due to non-neurogenic detrusor overactivity and lowered bladder capacity) in female rats. Administration of a selective serotonin reuptake inhibitor (SSRI) reversed urinary symptoms ${ }^{67}$. In humans, duloxetine, a serotoninnoradrenaline reuptake inhibitor (SNRI) improved frequency and urgency in female OAB patients significantly ${ }^{68}$.

Another possible explanation may be shared dysregulation of the hypothalamicpituitary-adrenal (HPA) axis resulting in increased corticotroponin-releasing factor (CRF), increased adrenocorticotropic hormone (ACTH) and increased cortisol; the HPA-axis is thought to play a role in depression and anxiety disorders ${ }^{63,69,70}$ and there is mounting evidence that CRF is involved in bladder function as well. In rats, repeated psychological distress resulted in increased urinary frequency and lower voiding volume ${ }^{71}$. CRF stimulated bladder function (lowered micturition threshold and lower urine volume) in a rodent model of detrusor overactivity and anxiety and administration of a CRF antagonist reduced detrusor overactivity ${ }^{72}$. Additionally several investigations from functional brain magnetic resonance imaging (MRI) during urodynamic studies revealed that increased brain activation in the anterior cingulate cortex (ACC) was associated with increased urgency at large bladder volumes, especially in young females with UI and detrusor overactivity ${ }^{73-75}$. Accordingly in young people with depressive disorder increased activity levels were found in the ACC. The ACC is part of a neural stress-network, which

is involved in (social) pain aspects ${ }^{76,77}$. Single photon emission computed tomography (SPECT) of older patients with UI showed hypo-perfusion in the regions of the prefrontal cortex ${ }^{78}$. Similar hypo-perfusion in the ACC and the prefrontal cortex was also seen in 
patients with major depressive disorder ${ }^{79}$. These findings suggest that prefrontal brain regions and parts of the default mode network may mediate both affective disorders and functional somatic complaints, like OAB.

\section{Strengths and limitations of the review}

The validity of the conclusion of our systematic review is influenced by the quality of the included studies. A large part of the initially retrieved articles did not employ validated screening instruments for the assessment of affective symptoms. The exclusion of these articles minimized the amount of bias. The most important biases are present in the definition and selection of the study population and the data collection. The quality of evidence may differ across outcomes within one study. An outcome-level assessment of risk of bias was maintained, after assessing the risk of bias for the studies as a whole. In the longitudinal studies, associations in different directions were present: OAB patients developing depression/anxiety and/or depressed/anxious people developing $O A B$, or both, all compared with control subjects. Furthermore, it is difficult to assess the prevalence of affective symptoms across different groups, as most of the studies reported differences in mean scores and odds ratios rather than percentages. Moreover, there was substantial heterogeneity with regard to diagnostic assessment. Only a few studies controlled for confounding variables and performed multivariate analysis ${ }^{42}$. Risk of selection bias was kept to a minimum because two reviewers independently selected the articles to be in- or excluded. Only studies with adult subjects were included, this could have introduced some selection, affecting generalizability across all age groups. However, the overall conclusion stands in different age groups, strengthening our conclusions. Quality assessment and risk of bias assessment of the 43 included articles was independently conducted by two authors, to avoid information bias. Language restriction may have led to biased results. Although articles not published in English are more common to exclude, Egger et al. demonstrated that there was a trend to publish in English if results achieved statistical significance ${ }^{80}$. On the other hand, the same authors concluded that language bias had no real effect on summary effect estimates most of the time. Thus, language restriction may possibly induce publication bias.

The most recent definition of OAB was launched in 2002. Three studies were published before $2002^{20-22}$. The definition of OAB may be less precise before that date. However, the current ICS definition is still not very specific, leaving the diagnosis of OAB heterogeneous. For instance, in research practice, different cut-off values concerning frequency were used. Thus, patients with $O A B$ according to the ICS definition remain to be a heterogeneous group. Consecutively, Lee et al. suggested that there is disagreement among physicians in the way $O A B$ is defined ${ }^{81}$. A more defined specification of the ICS definition is needed, since diagnosing multi-conditional disorders may be affected by under detection ${ }^{82}$. Furthermore, some of the excluded studies focussed on quality of life (QoL) only. This is a broad category, in which affective aspects are hard to specify. 
These studies showed that subjects with $O A B$ had significantly lower scores on QoL scales (mostly used: Short Form Health Survey (SF-36)) ${ }^{83-85}$. Some longitudinal research even showed that patients with low QoL scores, tended to develop OAB significantly more often ${ }^{84,85}$. In order to strengthen the conclusions found in OAB literature in the area of psychological wellbeing, psychological dimensions need to be specified more precisely, particularly with regard to affective conditions. Studies in men did not correct (sufficiently) for obstruction induced $O A B$ in (elderly) men e.g. ${ }^{51,62}$, thus making the results not automatically generalizable for men.

\section{Future research and implications}

Currently there are no on-going studies listed in the clinical trial database "ClinicalTrials.gov" to further explore the association of affective disorders with OAB. Thus, there is need for well-designed studies in order to clarify the exact nature of this association. Relevant pathophysiological mechanisms need to be integrated in these studies. Furthermore, a standardised, but also more personalized diagnostic and treatmentevaluation tool is required. The Experience Sampling Method (ESM), applied using an mhealth device, may fulfil this need. By exploring subjective reactions in daily life to experiences as they occur in the natural environment a better understanding of the relation between perceived stress and bladder function would be possible. ESM research in the area of affective conditions is promising ${ }^{86}$.

Considering the shortcomings of care management in patients with $\mathrm{OAB}{ }^{6}$ and given the strong association with affective symptoms, which are associated with treatment non-adherence ${ }^{87}$, an integrated approach involving urology, (uro-)gynaecology and psychiatry is recommended.

\section{Conclusions}

The results of this systematic review reveal a positive association between the cooccurrence of $O A B$ and affective symptoms. Furthermore, there is evidence for new onset of $O A B$ in depressive subjects, but further research is necessary to examine the strength of the effect. However, many evaluated studies relied on a single survey instrument, failed to note longitudinal changes and lacked information allowing assessment of epidemiological criteria for causality. Affective dysregulation may occasion worse OAB symptoms and poorer treatment outcome. Results also indicate that mental health professionals would do well to take into account urological symptoms in patients with affective complaints. The precise relationship of $O A B$ with affective conditions, is difficult to establish, but may involve a mechanism in the "bladder-brain-axis". Further research is needed in order to determine the direction of the association and the underlying pathophysiological pathways. 


\section{REFERENCES}

1. Disorders, D.a.S.M.o.M., DSM-IV. 4th Edition ed. 2000.

2. Wang, P.S., et al., Twelve-month use of mental health services in the United States: results from the National Comorbidity Survey Replication. Arch Gen Psychiatry, 2005. 62(6): p. 629-40.

3. Ustun, T.B., et al., Global burden of depressive disorders in the year 2000. Br J Psychiatry, 2004. 184: p. 386-92.

4. Abrams, P., et al., The standardisation of terminology of lower urinary tract function: Report from the standardisation sub-committee of the International Continence Society. Neurourology and Urodynamics, 2002. 21(2): p. 167-178.

5. Coyne, K.S., et al., National community prevalence of overactive bladder in the United States stratified by sex and age. Urology, 2011. 77(5): p. 1081-7.

6. Milsom, I., et al., How widespread are the symptoms of an overactive bladder and how are they managed? A population-based prevalence study. BJU Int, 2001. 87(9): p. 760-6.

7. Hu, T.W., et al., Estimated economic costs of overactive bladder in the United States. Urology, 2003. 61(6): p. 1123-8.

8. Tubaro, A., Defining overactive bladder: Epidemiology and burden of disease. Urology, 2004. 64(6 SUPPL. 1): p. 2-6.

9. Coyne, K.S., et al., Assessing patients' descriptions of lower urinary tract symptoms (LUTS) and perspectives on treatment outcomes: results of qualitative research. Int J Clin Pract, 2010. 64(9): p. 1260-78.

10. Coyne, K.S., et al., The impact of overactive bladder on mental health, work productivity and healthrelated quality of life in the UK and Sweden: results from EpiLUTS. BJU Int, 2011. 108(9): p. 1459-71.

11. Engel, W.J., Uropsychiatry. J Mich State Med Soc, 1964. 63: p. 273-7.

12. Frewen, W.K., Urgency incontinence. Review of 100 cases. J Obstet Gynaecol Br Commonw, 1972. 79.

13. Macaulay, A.J., et al., Micturition and the mind: psychological factors in the aetiology and treatment of urinary symptoms in women. Br Med J (Clin Res Ed), 1987. 294(6571): p. 540-3.

14. Cortes, E., et al., The psychology of LUTS: ICI-RS 2011. Neurourology and Urodynamics, 2012. 31(3): p. 340-343.

15. York, U.o. and C.f.r.a. dissemination. The overactive bladder and affective symptoms. CRD42014006641 2013; Available from: http://www.crd.york.ac.uk/PROSPERO/.

16. Moher, D., et al., Preferred reporting items for systematic reviews and meta-analyses: the PRISMA statement. J Clin Epidemiol, 2009. 62(10): p. 1006-12.

17. Centre for Evidence Based Medicine, U.o.O., US, Levels of Evidence, in Medical Sciences Division. March 2009.

18. Higgins, J.P., Cochrane Handbook for Systematic Reviews of Interventions 2011.

19. Guyatt, G.H., et al., GRADE guidelines: a new series of articles in the Journal of Clinical Epidemiology. J Clin Epidemiol, 2011. 64(4): p. 380-2.

20. Chiara, G., et al., Psychological investigation in female patients suffering from urinary incontinence. Int Urogynecol J Pelvic Floor Dysfunct, 1998. 9(2): p. 73-7.

21. Zorn, B.H., et al., Urinary incontinence and depression. J Urol, 1999. 162(1): p. 82-4.

22. Bodden-Heidrich, R., et al., Psychosomatic aspects of urinary incontinence. Archives of Gynecology and Obstetrics, 1999. 262(3-4): p. 151-158.

23. Rogers, R., et al., Efficacy of tolterodine on overactive bladder symptoms and sexual and emotional quality of life in sexually active women. Int Urogynecol J Pelvic Floor Dysfunct, 2008. 19(11): p. 1551-7.

24. Bradley, C.S., et al., Urinary incontinence, depression and posttraumatic stress disorder in women veterans. American Journal of Obstetrics and Gynecology, 2012. 206(6).

25. Ikeda, Y., et al., Risk factors for overactive bladder in the elderly population: a community-based study with face-to-face interview. Int J Urol, 2011. 18(3): p. 212-8. 


\section{Chapter 2}

26. Sexton, C.C., et al., Prevalence and effect on health-related quality of life of overactive bladder in older americans: results from the epidemiology of lower urinary tract symptoms study. J Am Geriatr Soc, 2011. 59(8): p. 1465-70.

27. Perry, S., C.W. McGrother, and K. Turner, An investigation of the relationship between anxiety and depression and urge incontinence in women: Development of a psychological model. British Journal of Health Psychology, 2006. 11(3): p. 463-482.

28. Song, H.J. and J.M. Bae, Prevalence of urinary incontinence and lower urinary tract symptoms for community-dwelling elderly 85 years of age and older. J Wound Ostomy Continence Nurs, 2007. 34(5): p. 535-41.

29. van der Vaart, C.H., et al., Association between urogenital symptoms and depression in communitydwelling women aged 20 to 70 years. Urology, 2007. 69(4): p. 691-6.

30. van de Pol, G., et al., Is there an association between depressive and urinary symptoms during and after pregnancy? Int Urogynecol J Pelvic Floor Dysfunct, 2007. 18(12): p. 1409-15.

31. Waetjen, L.E., et al., Association between menopausal transition stages and developing urinary incontinence. Obstetrics and Gynecology, 2009. 114(5): p. 989-998.

32. Woods, N.F. and E.S. Mitchell, Consequences of incontinence for women during the menopausal transition and early postmenopause: observations from the Seattle Midlife Women's Health Study. Menopause, 2013.

33. Stewart, W.F., et al., Prevalence and burden of overactive bladder in the United States. World J Urol, 2003. 20(6): p. 327-36.

34. Coyne, K.S., et al., The impact on health-related quality of life of stress, urge and mixed urinary incontinence. BJU Int, 2003. 92(7): p. 731-5.

35. Coyne, K.S., et al., The impact of overactive bladder, incontinence and other lower urinary tract symptoms on quality of life, work productivity, sexuality and emotional well-being in men and women: results from the EPIC study. BJU International, 2008. 101(11): p. 1388-1395.

36. Milsom, I. and D.E. Irwin, A Cross-Sectional, Population-Based, Multinational Study of the Prevalence of Overactive Bladder and Lower Urinary Tract Symptoms: Results from the EPIC Study. European Urology, Supplements, 2007. 6(1): p. 4-9.

37. Coyne, K.S., et al., Urinary incontinence and its relationship to mental health and health-related quality of life in men and women in Sweden, the United Kingdom, and the United States. Eur Urol, 2012. 61(1): p. 88-95.

38. Coyne, K.S., et al., The burden of lower urinary tract symptoms: evaluating the effect of LUTS on healthrelated quality of life, anxiety and depression: EpiLUTS. BJU Int, 2009. 103 Suppl 3: p. 4-11.

39. Milsom, I., et al., Effect of bothersome overactive bladder symptoms on health-related quality of life, anxiety, depression, and treatment seeking in the United States: results from EpiLUTS. Urology, 2012. 80(1): p. 90-6.

40. Melville, J.L., et al., Prevalence of comorbid psychiatric illness and its impact on symptom perception, quality of life, and functional status in women with urinary incontinence. Am J Obstet Gynecol, 2002. 187(1): p. 80-7.

41. Sung, V.W., et al., Association between urinary incontinence and depressive symptoms in overweight and obese women. Am J Obstet Gynecol, 2009. 200(5): p. 557 e1-5.

42. Felde, G., I. Bjelland, and S. Hunskaar, Anxiety and depression associated with incontinence in middleaged women: A large Norwegian cross-sectional study. International Urogynecology Journal and Pelvic Floor Dysfunction, 2012. 23(3): p. 299-306.

43. Ito, T., et al., Is Major Depression a Risk for Bladder, Bowel, and Sexual Dysfunction? Luts-Lower Urinary Tract Symptoms, 2012. 4(2): p. 87-95.

44. Tettamanti, G., et al., Depression, Neuroticism, and Urinary Incontinence in Premenopausal Women: A Nationwide Twin Study. Twin Res Hum Genet, 2013: p. 1-8.

45. Sims, J., et al., Urinary incontinence in a community sample of older adults: prevalence and impact on quality of life. Disabil Rehabil, 2011. 33(15-16): p. 1389-98. 
46. Nuotio, M., et al., Six-year follow-up and predictors of urgency-associated urinary incontinence and bowel symptoms among the oldest old: a population-based study. Arch Gerontol Geriatr, 2009. 49(2): p. e8590.

47. Hirayama, A., et al., Risk factors for new-onset overactive bladder in older subjects: results of the Fujiwara-kyo study. Urology, 2012. 80(1): p. 71-6.

48. Das, A.K., et al., Improvement in depression and health-related quality of life after sacral nerve stimulation therapy for treatment of voiding dysfunction. Urology, 2004. 64(1): p. 62-8.

49. Ozdedeli, S., H. Karapolat, and Y. Akkoc, Comparison of intravaginal electrical stimulation and trospium hydrochloride in women with overactive bladder syndrome: a randomized controlled study. Clin Rehabil, 2010. 24(4): p. 342-51.

50. Kafri, R., et al., Depressive symptoms and treatment of women with urgency urinary incontinence. Int Urogynecol J, 2013. nov;24(11): p.1953-9

51. Staskin, D.R., et al., Effects of oxybutynin transdermal system on health-related quality of life and safety in men with overactive bladder and prostate conditions. Int J Clin Pract, 2008. 62(1): p. 27-38.

52. Stach-Lempinen, B., et al., Severe depression determines quality of life in urinary incontinent women. Neurourol Urodyn, 2003. 22(6): p. 563-8.

53. Knight, S., et al., Comparisons of pelvic floor muscle performance, anxiety, quality of life and life stress in women with dry overactive bladder compared with asymptomatic women. BJU Int, 2012. 109(11): p. 1685-9.

54. Avery, J.C., et al., Identifying the quality of life effects of urinary incontinence with depression in an Australian population. BMC Urol, 2013. 13: p. 11.

55. Hullfish, K.L., et al., Postpartum depression, urge urinary incontinence, and overactive bladder syndrome: is there an association? Int Urogynecol J Pelvic Floor Dysfunct, 2007. 18(10): p. 1121-6.

56. Gerst, K., et al., Self-reported urge urinary incontinence (UUI) among older Mexican-American men: risk factors and psycho-social consequences. J Immigr Minor Health, 2011. 13(6): p. 1110-5.

57. Jackson, R.A., et al., Urinary incontinence in elderly women: Findings from the health, aging, and body composition study. Obstetrics and Gynecology, 2004. 104(2): p. 301-307.

58. Melville, J.L., et al., Incontinence severity and major depression in incontinent women. Obstet Gynecol, 2005. 106(3): p. 585-92.

59. Palmer, M.H., et al., Urinary incontinence and overactive bladder in patients with heart failure. J Urol, 2009. 182(1): p. 196-202.

60. Tennstedt, S.L., et al., Prevalence of and risk factors for urine leakage in a racially and ethnically diverse population of adults: the Boston Area Community Health (BACH) Survey. Am J Epidemiol, 2008. 167(4): p. 390-9.

61. Lim, J.R., C.W. Bak, and J.B. Lee, Comparison of anxiety between patients with mixed incontinence and those with stress urinary incontinence. Scand J Urol Nephrol, 2007. 41(5): p. 403-6.

62. Hirayama, A., et al., Risk factors for new onset of overactive bladder in older subjects: Results of the fujiwara kyo study. Neurourology and Urodynamics, 2012. 31 (6): p. 765-766.

63. Nemeroff, C.B., The neurobiology of depression. Sci Am, 1998. 278(6): p. 42-9.

64. Gordon, J.A. and R. Hen, The serotonergic system and anxiety. Neuromolecular Med, 2004. 5(1): p. 2740.

65. Steers, W., Potential targets in the treatment of urinary incontinence. Reviews in urology, 2001. 3 Suppl 1: p. S19-26.

66. de Groat, W.C., Influence of central serotonergic mechanisms on lower urinary tract function. Urology, 2002. 59(5 Suppl 1): p. 30-6.

67. Lee, K.S., et al., Alterations in voiding frequency and cystometry in the clomipramine induced model of endogenous depression and reversal with fluoxetine. Journal of Urology, 2003. 170(5): p. 2067-2071.

68. Steers, W.D., et al., Duloxetine compared with placebo for treating women with symptoms of overactive bladder. BJU Int, 2007. 100(2): p. 337-45.

69. Arborelius, L., et al., The role of corticotropin-releasing factor in depression and anxiety disorders. J Endocrinol, 1999. 160(1): p. 1-12. 
70. Nemeroff, C.B., Recent advances in the neurobiology of depression. Psychopharmacol Bull, 2002. 36 Suppl 2: p. 6-23.

71. Smith, A.L., et al., The effects of acute and chronic psychological stress on bladder function in a rodent model. Urology, 2011. 78(4): p. 967.e1-967.e7.

72. Klausner, A.P., et al., The role of corticotropin releasing factor and its antagonist, astressin, on micturition in the rat. Autonomic Neuroscience: Basic and Clinical, 2005. 123(1-2): p. 26-35.

73. Griffiths, D., Imaging bladder sensations. Neurourol Urodyn, 2007. 26(6 Suppl): p. 899-903.

74. Griffiths, D. and S.D. Tadic, Bladder control, urgency, and urge incontinence: Evidence from functional brain imaging. Neurourology and Urodynamics, 2008. 27(6): p. 466-474.

75. Komesu, Y.M., et al., Functional MRI of the Brain in Women with Overactive Bladder: Brain Activation During Urinary Urgency. Female Pelvic medicine and reconstructive surgery, 2011. 30(1542-5983 (Electronic)): p. 3.

76. Lederbogen, F., et al., City living and urban upbringing affect neural social stress processing in humans. Nature, 2011. 474(7352): p. 498-501.

77. Eisenberger, N.I., The neural bases of social pain: evidence for shared representations with physical pain. Psychosom Med, 2012. 74(2): p. 126-35.

78. Griffiths, D., Clinical studies of cerebral and urinary tract function in elderly people with urinary incontinence. Behav Brain Res, 1998. 92(2): p. 151-5.

79. Awata, S., et al., Regional cerebral blood flow abnormalities in late-life depression: relation to refractoriness and chronification. Psychiatry Clin Neurosci, 1998. 52(1): p. 97-105.

80. Egger, M., et al., Language bias in randomised controlled trials published in English and German. Lancet, 1997. 350(9074): p. 326-9.

81. Lee, H.E., et al., Short-term effects of a systematized bladder training program for idiopathic overactive bladder: A prospective study. International Neurourology Journal, 2013. 17(1): p. 11-17.

82. Leue, C., et al., Managing complex patients on a medical psychiatric unit: an observational study of university hospital costs associated with medical service use, length of stay, and psychiatric intervention. J Psychosom Res, 2010. 68(3): p. 295-302.

83. Araki, I., et al., Prevalence and impact on generic quality of life of urinary incontinence in Japanese working women: assessment by ICl questionnaire and SF-36 Health Survey. Urology, 2005. 66(1): p. 88-93.

84. Tadic, S.D., et al., Effect of biofeedback on psychological burden and symptoms in older women with urge urinary incontinence. Journal of the American Geriatrics Society, 2007. 55(12): p. 2010-2015.

85. Cheung, R.Y.K., et al., Quality of life in women with urinary incontinence is impaired and comparable to women with chronic diseases. Hong Kong Medical Journal, 2012. 18(3): p. 214-220.

86. Kramer, I., et al., A therapeutic application of the experience sampling method in the treatment of depression: a randomized controlled trial. World Psychiatry, 2014. 13(1): p. 68-77.

87. Kondryn, H.J., et al., Treatment non-adherence in teenage and young adult patients with cancer. Lancet Oncol, 2011. 12(1): p. 100-8.

88. Beck, A.T., et al., Comparison of Beck Depression Inventories -IA and -II in psychiatric outpatients. J Pers Assess, 1996. 67(3): p. 588-97.

89. Boyd, J.H., et al., Screening for depression in a community sample. Understanding the discrepancies between depression symptom and diagnostic scales. Arch Gen Psychiatry, 1982. 39(10): p. 1195-200.

90. Yesavage, J.A., et al., Development and validation of a geriatric depression screening scale: a preliminary report. J Psychiatr Res, 1982. 17(1): p. 37-49.

91. Friedman, B., M.J. Heisel, and R.L. Delavan, Psychometric properties of the 15-item geriatric depression scale in functionally impaired, cognitively intact, community-dwelling elderly primary care patients. J Am Geriatr Soc, 2005. 53(9): p. 1570-6.

92. Zigmond, A.S. and R.P. Snaith, The hospital anxiety and depression scale. Acta Psychiatr Scand, 1983. 67(6): p. 361-70.

93. Spitzer, R.L., K. Kroenke, and J.B. Williams, Validation and utility of a self-report version of PRIME-MD: the PHQ primary care study. Primary Care Evaluation of Mental Disorders. Patient Health Questionnaire. JAMA, 1999. 282(18): p. 1737-44. 
94. Haro, J.M., et al., Concordance of the Composite International Diagnostic Interview Version 3.0 (CIDI 3.0) with standardized clinical assessments in the WHO World Mental Health surveys. Int J Methods Psychiatr Res, 2006. 15(4): p. 167-80.

95. Cox, J.L., J.M. Holden, and R. Sagovsky, Detection of postnatal depression. Development of the 10-item Edinburgh Postnatal Depression Scale. Br J Psychiatry, 1987. 150: p. 782-6.

96. Hamilton, M., A rating scale for depression. J Neurol Neurosurg Psychiatry, 1960. 23: p. 56-62.

97. Jorm, A.F., et al., The psychogeriatric assessment scales (PAS): further data on psychometric properties and validity from a longitudinal study of the elderly. Int J Geriatr Psychiatry, 1997. 12(1): p. 93-100.

98. Robins, L.N., et al., National Institute of Mental Health Diagnostic Interview Schedule. Its history, characteristics, and validity. Arch Gen Psychiatry, 1981. 38(4): p. 381-9.

99. Hamilton, M., The assessment of anxiety states by rating. Br J Med Psychol, 1959. 32(1): p. 50-5.

100. Beck, A.T., et al., An inventory for measuring clinical anxiety: psychometric properties. J Consult Clin Psychol, 1988. 56(6): p. 893-7.

101. Kvaal, K., et al., The Spielberger State-Trait Anxiety Inventory (STAl): the state scale in detecting mental disorders in geriatric patients. Int J Geriatr Psychiatry, 2005. 20(7): p. 629-34.

102. Guyatt, G., et al., GRADE guidelines: 1. Introduction-GRADE evidence profiles and summary of findings tables. J Clin Epidemiol, 2011. 64(4): p. 383-94. 


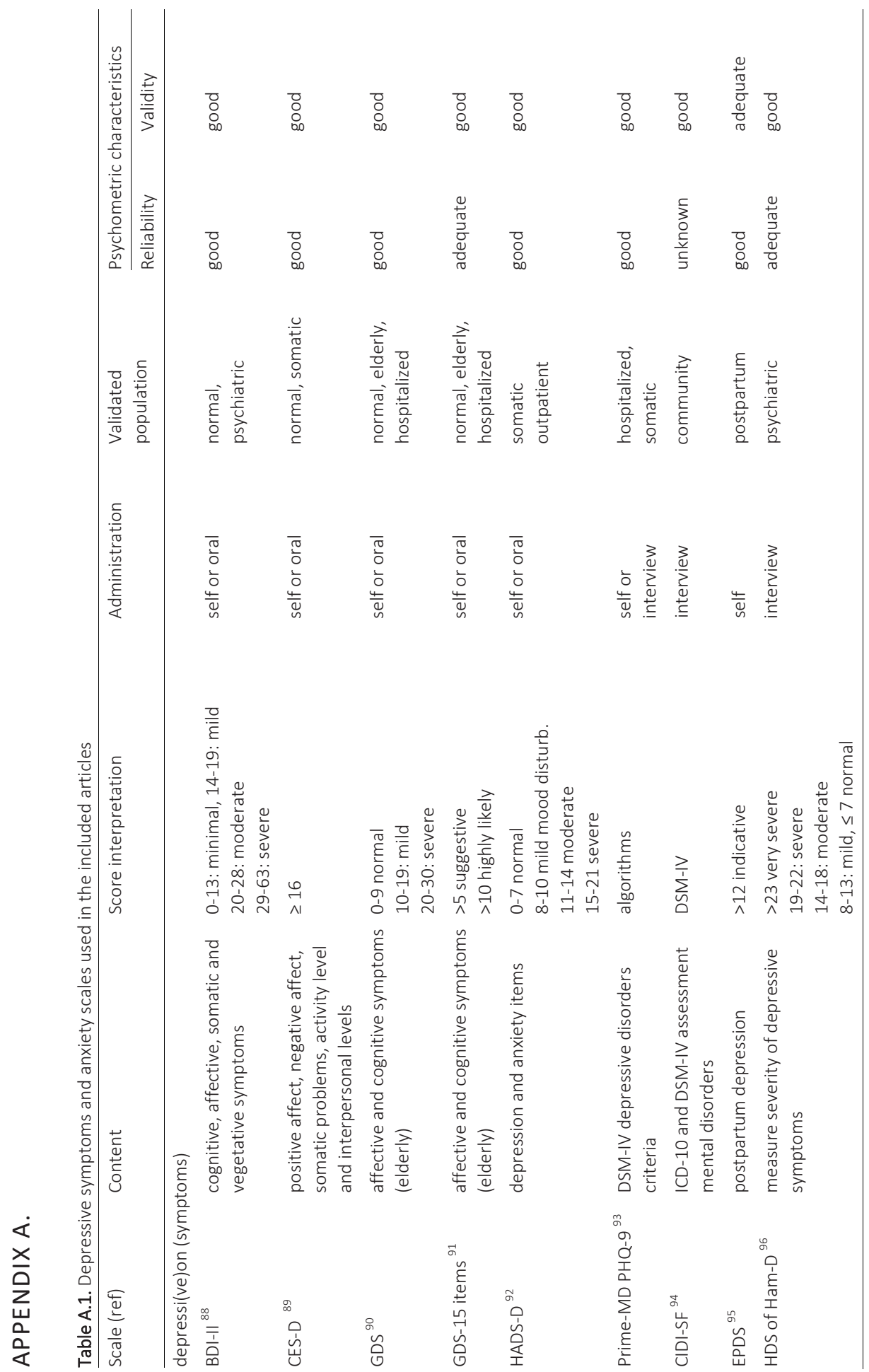


Affective symptoms and $O A B$ - a systematic review

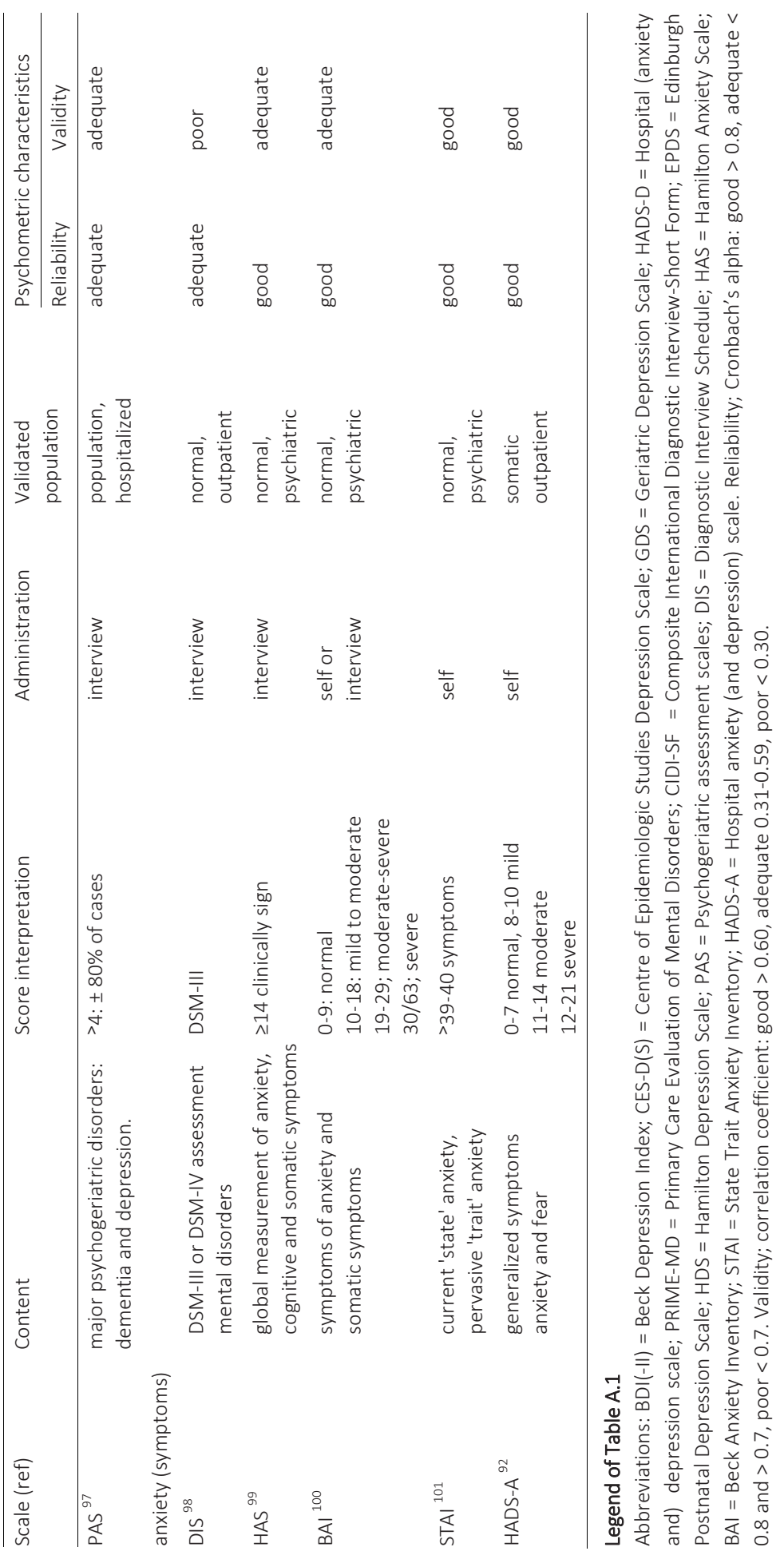




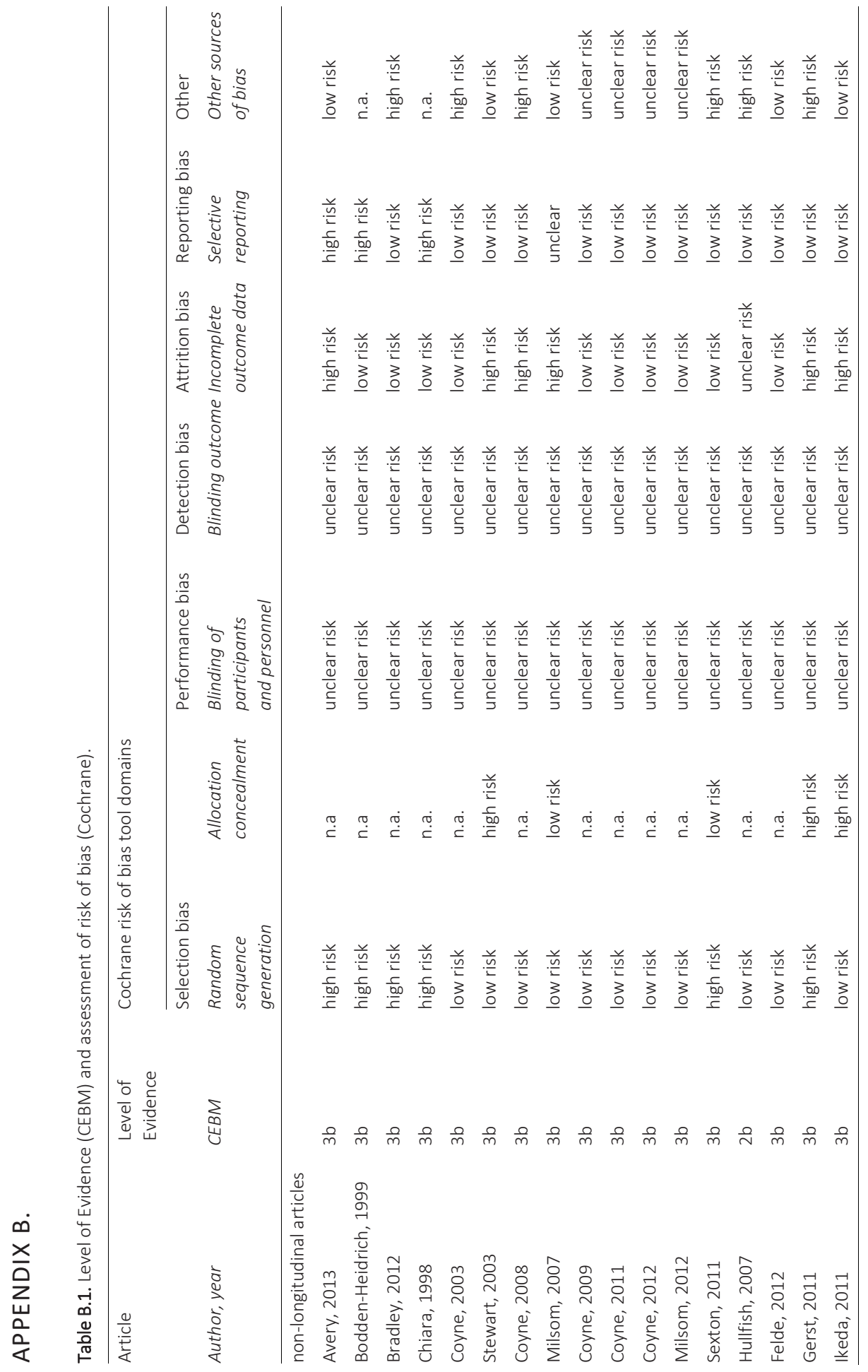




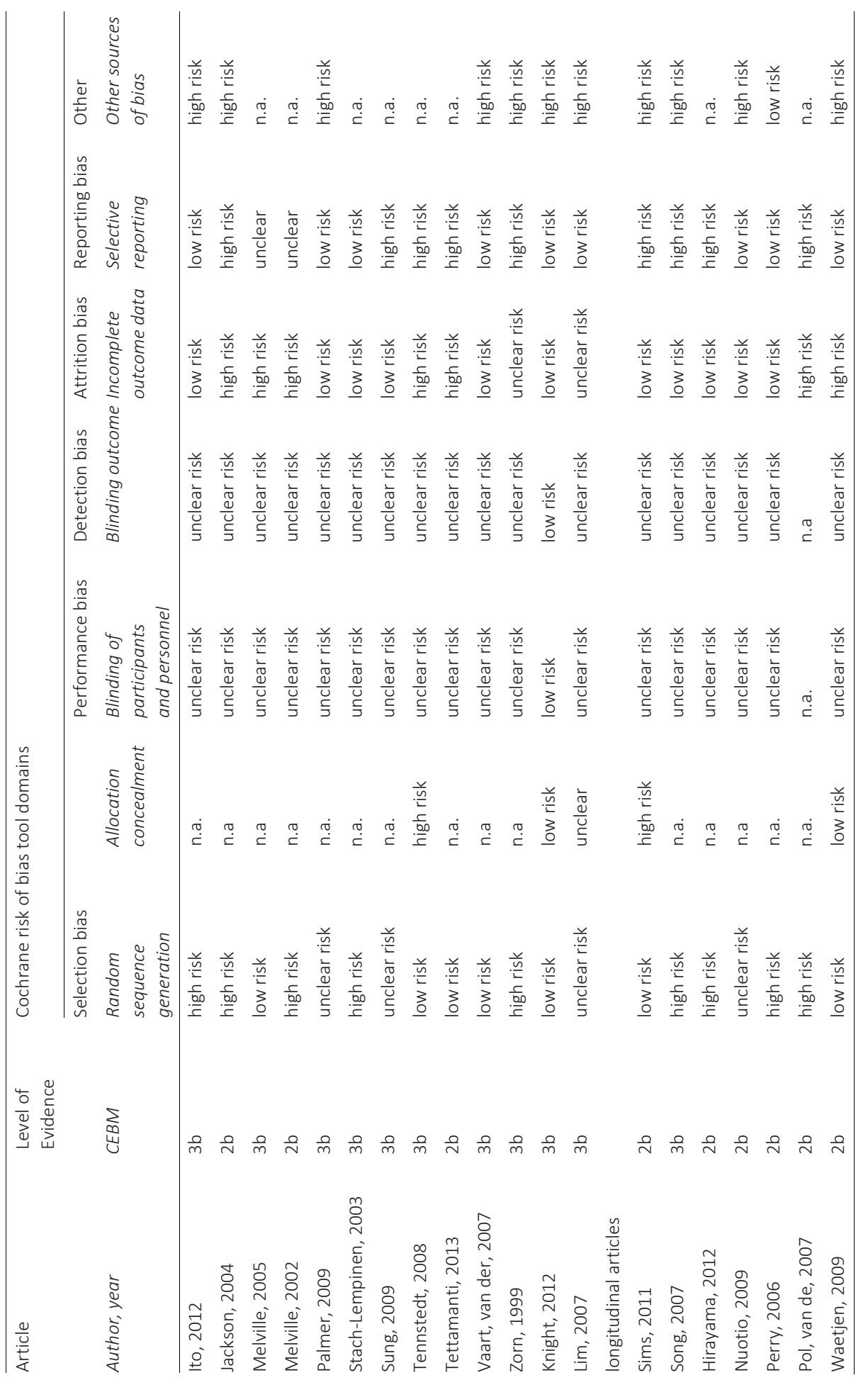


Chapter 2

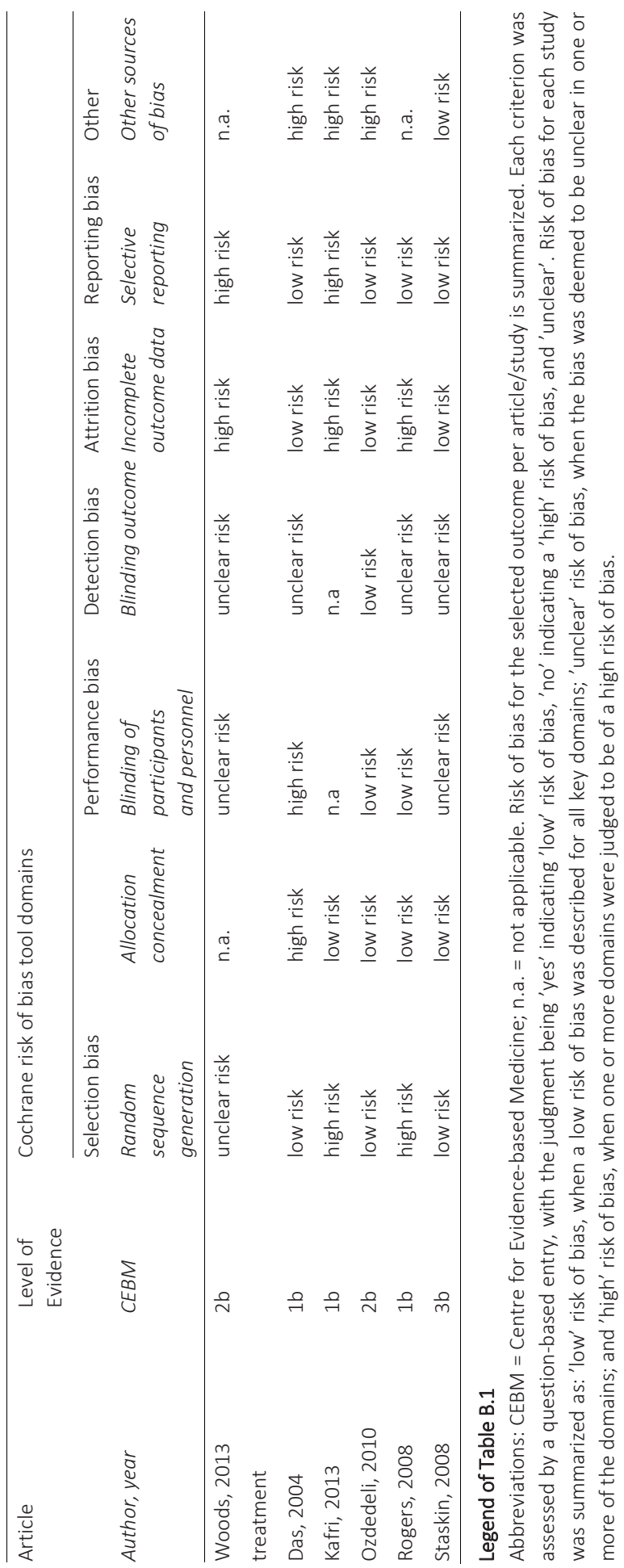




\section{APPENDIX C.}

\section{C.1 Literature search}

Detrusor overactivity, bladder overactivity, urinary bladder overactive, overactive bladder (syndrome), overactive detrusor function, overactive detrusor, urinary incontinence urge, urinary urge incontinence, OAB, urge incontinence, urgency/frequency (syndrome), urinary urgency/frequency (syndrome), urinary urgency incontinence was combined with depression, depressive disorder, depressive traits, depressive symptoms, emotional depression, anxiety, anxiety disorder, anxiety symptoms, anxiety traits, generalized anxiety disorder, panic disorder, Hospital anxiety and depression scale, Hamilton anxiety scale, Beck depression scale, Center for epidemiological studies depression scale, affective disorder, affective symptoms, mental health.

\section{APPENDIX D.}

\section{D.1 GRADE Approach}

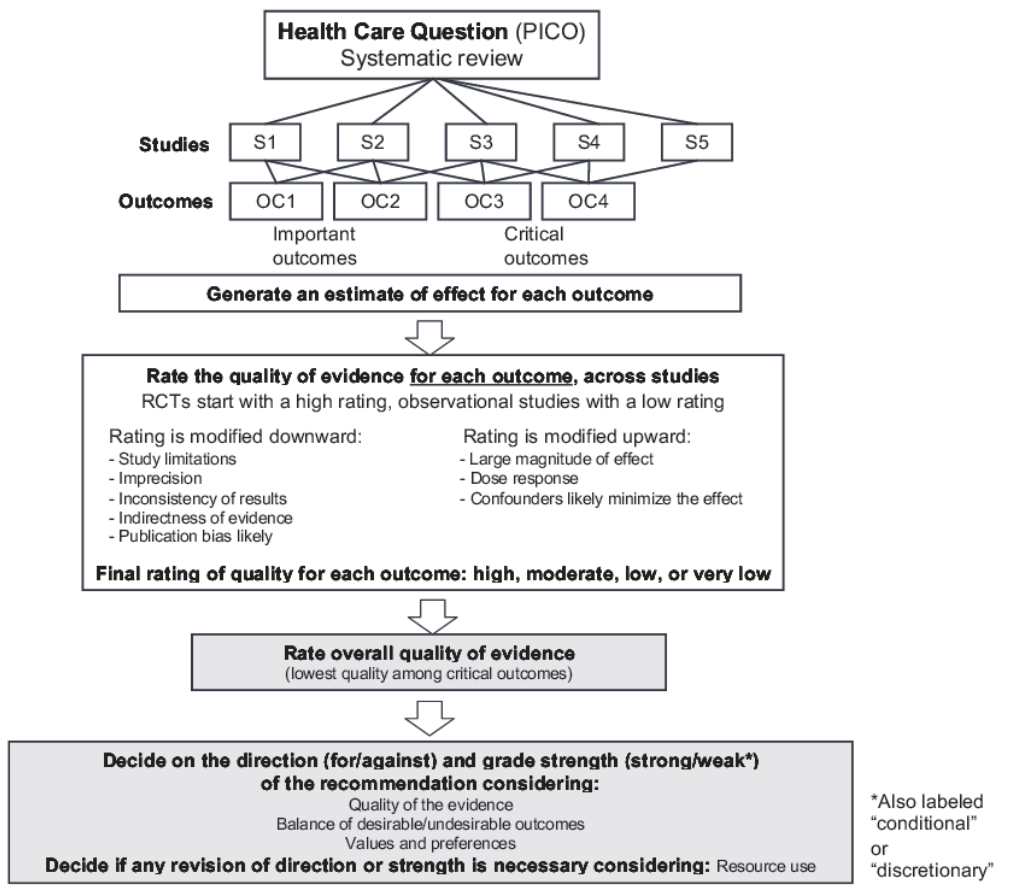

Figure. D.1. Schematic view of GRADE approach. Abbreviation: RCT: randomized controlled trials. Reprinted with permission from G. Guyatt et al. $2011^{102}$. 



\section{Chapter}

\section{Prevalence of anxiety and depressive symptoms and their association with pelvic floor dysfunctions - a cross sectional cohort study at a Pelvic Care Centre}

Vrijens DMJ ${ }^{*}$, Berghmans B ${ }^{*}$, Nieman F, van Os J, van Koeveringe GA, C. Leue Neurourol and Urodyn. 2017 Febr 21, (Epub ahead of print)

${ }^{*}$ Both authors contributed equally 


\section{ABSTRACT}

\section{Aims}

Pelvic floor dysfunctions (PFDs), like voiding complaints, urinary and faecal incontinence and prolapse, are prevalent and associated with decrease in quality of life. PFDs are often complex and multifactorial in origin, showing interrelationships between different PFD and with affective conditions. The primary aim of this study is to describe the prevalence of affective complaints in a cohort of Pelvic Care Centre (PCC) patients. The secondary aim is to describe associations between PFDs and depression or anxiety.

\section{Methods}

A cross sectional cohort study at a University Hospital's PCC. First contact patients were included in a triage system and filled out questionnaires regarding pelvic floor complaints and Hospital Anxiety and Depression Scale (HADS) scores. Linear (dummy-) regression analysis of HADS scales was performed to test the effects of relevant clinical predictors related, and not directly related, to pelvic floor problems, and demographic characteristics of the patients.

\section{Results}

From 1862 eligible first-contact PCC patients 1510 (mean age 57.1) had completed the questionnaire (352 missing, 18.9\%). The prevalence of anxiety and depression complaints was 30.9 and $20.3 \%$, respectively. The variance explained for depression score by PFDs was 0.12 and 0.074 for anxiety.

\section{Conclusion}

Anxiety and depression are prevalent (30.9\% and 20.3\%, respectively) in a cohort of PFDs. PFDs can explain variance within anxiety and depression complaints. Corrected for other contributing variables, $12 \%$ of depression and $7.4 \%$ of anxiety was directly related to PFDs. We advocate a multidisciplinary approach, containing psychometric assessment for PFDs in order to obtain better diagnostic results and personalized treatment options. 


\section{INTRODUCTION}

Pelvic floor dysfunctions (PFDs), such as voiding complaints, urinary and faecal incontinence (UI, FI), constipation, sexual problems and pelvic organ prolapse (POP) are prevalent and often distressing health problems in both sexes ${ }^{1}$. The incidence increases with age and PFDs are associated with a decrease in quality of life ${ }^{1}$. Life expectancy of people is rising and therefore the worldwide burden of PFDs is expected to increase significantly over time. Therefore future requirements for PFD-related healthcare is expected to rise by $50 \%$ over the next 30 years ${ }^{2}$. PFDs are complex and multi-factorial by origin, therefore a multidisciplinary approach is recommended ${ }^{3}$.

Recently, we reported about the complexity of PFDs presented at our Pelvic Care Centre (PCC), their multi-factorial character and frequent interactions between each other. We demonstrated that one out of four women and one out of seven male patients had more than one pelvic floor related health problem ${ }^{4}$. Overactive bladder $(\mathrm{OAB})$ is such a complex PFD, with a prevalence of around $17 \%{ }^{5}$. OAB is a symptom complex of urgency (sudden compelling desire to pass urine, which is difficult to defer), usually with frequency (voiding 8 or more times per day) and nocturia (awakening at night to void), with (OAB wet) or without ( $O A B$ dry) urgency urinary incontinence ${ }^{6}$. OAB with or without incontinence is often associated with affective disorders ${ }^{7}$, and may lead to significant social and functional impairment ${ }^{8}$. OAB and affective disorders may even share common biological pathways comparable to functional bowel disorders ${ }^{9}$.

Affective disorders such as anxiety disorders or mood disorders, according to the DSM-V criteria ${ }^{10,11}$ have a high lifetime prevalence ranging from 2-15\% for depression ${ }^{11}$ and around $18 \%$ for anxiety disorders ${ }^{12}$. Anxiety disorders are globally the most common psychiatric disorders according to the World Health Organisation 11, ${ }^{12}$. However, most physicians, do not adequately identify affective conditions based on self-reported symptoms ${ }^{12}$ similar to what has been reported for irritable bowel syndrome (IBS) ${ }^{13}$ For example, physicians miss the diagnosis of somatoform disorders in around two third of the cases, and psychiatric diagnoses remained undetected in even $95 \%$ of most complex patients with somatic and psychiatric com-morbidity ${ }^{14,15}$.

Our aim is to describe, analyse and interpret the relation between PFD symptoms and Hospital Anxiety and Depression Scale (HADS) scores in patients referred to our PCC.

\section{MATERIALS AND METHODS}

The PCC where the study was conducted is a multidisciplinary outpatient clinic with participation of urologists, gynaecologists, colorectal surgeons, gastro-enterologists, general hospital psychiatrists, pelvic physiotherapists, sexologists and specially trained continence nurses. Since 2005 , it is serving as a large secondary and tertiary referral 
centre, and more than 5500 patients with functional pelvic disorders have been treated yet. The PCC developed and used a digital triage system for patient selection, in order to measure subjective complaints during the preceding six months with regard to 6 different PFDs ( i.e. voiding dysfunction, UI, POP, constipation, FI, and sexual/pelvic pain problems). The triage has been described elsewhere ${ }^{4}$. In 2008, our PCC team started collaboration with the hospital psychiatrists. One of the reasons was that at the PCC many PFDs patients - especially those with frequent and recurrent visits - were seen with symptoms of psychological and psychiatric ill-health. Hence, it was decided to routinely screen PCC patients by using the HADS questionnaire ${ }^{16}$. The HADS is a short self-assessment tool to screen for the presence of a mood disorder in medically ill patients. It contains 14 items, each rated on a 4-point Likert scale, which are divided into two subscales that assess depression (HADS-D) and anxiety (HADS-A). The subscales are categorized as a four level ordinal variable: normal 0-7; mild 8-10, moderate 11-14 or severe mood disturbance 15-21 The HADS has a high internal consistency (Cronbach's alpha $=0.85)^{16}$. From 2008 to 2013, all first contact PCC patients with PFD were included in the present study.

\section{STATISTICS}

Statistics of categorical data are presented as frequencies and percentages. Deviations from normality of distribution in metric variables were tested by the Shapiro-Wilk test. If metric data were not-normally distributed minimum and maximum values are added to the mean and the standard deviation. In order to facilitate a parametric statistical analysis it was decided to calculate the square root of the two outcome scores (the HADS anxiety and depression scales) and to use the transformation as separate dependent variables in regression analysis. A residual analysis was performed for each outcome to investigate whether the distribution of Studentized z-score residuals turns out to be not too dissimilar from a normal distribution. Linear (dummy-)regression analysis of HADS depression and HADS anxiety scales was done to test the effects of A: relevant clinical predictors related to pelvic floor problems, B: other relevant clinical predictors not directly related to pelvic floor problems and C: demographic characteristics of the patients. Section A of the predictors was subdivided into six general factors measuring prevalence of problems in the pelvic region : urinary incontinence, voiding problems, prolapse, faecal incontinence, constipation and sexual problems, and into 40 specific potential predictors measuring more detailed items from a questionnaire concerning pelvic problems. At first, explorative regression models both forward selection and backward elimination techniques with list wise deletion of missing values were used. It was decided that section $C$ predictors (age and gender) plus the six general prevalence predictors from section $A$ always had to be included within the search for the best-fitting model, regardless whether they would have statistical significant effects 
upon the outcome or not. Next, the resulting direct-effects regression model was tested for all first-order interactions using forward selection only. Next to variance explained by the model, the additional variance explained by pelvic predictors is given. A p-value of $<0.05$ is considered to be statistically significant. All data-analysis was done with IBM SPSS, pc version 22.

\section{RESULTS}

From the 1862 eligible first-contact PCC patients, 1510 satisfactorily completed the HADS questionnaire (352 missing, 18.9\%). Mean age of patients was 57.1 , standard deviation (s.d.). 16.9 (14-94), and 1543 (82.9\%) were female patients, while 319 (17.1\%) were men. Table 1 shows - next to the prevalence of first contact pelvic floor complaints - the clinical background variables, which were also presumed to have an effect on depression and/or anxiety. Table 2 presents the statistical characteristics of both main outcome variables: HADS anxiety and HADS depression scores concerning the four severity categories of both outcomes for the total group and for men and women separately. More than $30 \%$ of the patients had an abnormal anxiety score and more than $20 \%$ presented an abnormal depression score. Testing for normality of distribution, it was found that both parameters were non-normally distributed. The Shapiro-Wilk test results for HADS-anxiety and HADS-depression respectively is $p<0.001$.

Within successive explorative regression models the final best-fitting model predicted the HADS depression and anxiety. A final regression model for each main outcome is presented in Tables 3 and table 4. Table 3 shows the results of the final (dummy) regression model for HADS-depression. Eight direct regression effects and three firstorder interactions had statistically significant effects upon HADS depression. Final results can be summarized as follows, interpreting the presented unstandardized regression coefficients. 1. (0.008) The higher the age of a patient, the higher the HADS depression scores are. 2. (0.220) If patients responded affirmatively on having urine incontinence, they showed higher scores on the HADS depression sub-score. 3. (0.460) If patients responded affirmatively on having sexual problems, they showed higher scores on HADS depression. 4. (0.066) If patients confirmed to have complaints about the composition of their faeces, they tended to have higher scores on HADS depressive symptoms. 5. (0.156) If patients suffered from serious headaches, they had higher scores on HADS depression sub-score. 6. (0.049) If patients showed complaints on bladder emptying during the past 6 months, they had higher scores on HADS depression. 7 (0.056) If patients said that they had a burning sensation or pain while urinating in the past 6 months, they were likely to score higher on HADS depression. 8. (0.101) If patients had diabetes mellitus, they also showed high scores on HADS depression. 9. (0.284) If patients confirmed that they had prolapse-related voiding problems, they showed high scores on HADS depressive symptoms. 10. (0.366) Faecal incontinence in 
men had higher effects on HADS depression scores than the faecal incontinence in women. 11. (-0.145) The higher the involuntary loss of urine during 24 hours, the higher the score on HADS depression sub-score is. This effect is significantly higher in female patients compared to men. The explained variance for the final regression model in 1506 patients is 0.120 .

Table 1. Demographic background variables and clinical characteristics specific for problems or complaints within the pelvic region in patients contacted for the first time at the PCC during 2008-2013 (N=1862).

\begin{tabular}{ll}
\hline Gender & female: $1543(82.9 \%)$ male: $319(17.1 \%)$ \\
Age & 56.1 years (s.d. 16.9 range: $14-94)$ \\
\hline
\end{tabular}

\begin{tabular}{|c|c|c|c|c|}
\hline \multicolumn{5}{|c|}{ Complaints of (now or during the past year) } \\
\hline Asthma & 223 & \multicolumn{3}{|l|}{$(12.0 \%)$} \\
\hline High blood pressure & 414 & \multicolumn{3}{|l|}{$(22.2 \%)$} \\
\hline Gall stones & 25 & \multicolumn{3}{|l|}{$(1.3 \%)$} \\
\hline Kidney stones & 15 & \multicolumn{3}{|l|}{$(0.8 \%)$} \\
\hline Diabetes & 135 & \multicolumn{3}{|l|}{$(7.3 \%)$} \\
\hline Headaches & 139 & \multicolumn{3}{|l|}{$(7.5 \%)$} \\
\hline \multicolumn{5}{|l|}{ Prevalence of : } \\
\hline Urine incontinence & 726 & \multicolumn{3}{|l|}{$(39.0 \%)$} \\
\hline Voiding problems & 1106 & \multicolumn{3}{|l|}{$(59.4 \%)$} \\
\hline Prolapse & 501 & \multicolumn{3}{|l|}{$(26.9 \%)$} \\
\hline Obstipation & 179 & \multicolumn{3}{|l|}{$(9.6 \%)$} \\
\hline Faecal Incontinence & 261 & \multicolumn{3}{|l|}{$(14.0 \%)$} \\
\hline Sexual problems & 61 & \multicolumn{3}{|l|}{$(3.3 \%)$} \\
\hline \multirow[t]{2}{*}{ Uterus extirpation } & 315 & \multicolumn{3}{|l|}{$(26.4 \%)$} \\
\hline & & Sometimes & Often & Always \\
\hline \multicolumn{2}{|l|}{ Urinary urgency } & $169(9.1 \%)$ & $138(7.4 \%)$ & $180(9.7 \%)$ \\
\hline \multicolumn{2}{|c|}{ Stress incontinence (coughing) } & $241(12.9 \%)$ & $153(8.2 \%)$ & $99(5.3 \%)$ \\
\hline \multicolumn{2}{|c|}{ Burning pain during voiding } & $201(10.9 \%)$ & $107(5.4 \%)$ & $25(1.3 \%)$ \\
\hline \multicolumn{2}{|c|}{ Haematuria past 2 years } & $77(4.1 \%)$ & $17(0.9 \%)$ & $9(0.5 \%)$ \\
\hline \multicolumn{2}{|c|}{ Incomplete bladder emptying } & $3(0.3 \%)$ & $327(27.9 \%)$ & $291(24.9 \%)$ \\
\hline \multicolumn{2}{|c|}{ Abdominal pain past 6 months } & $9(6.9 \%)$ & $57(4.0 \%)$ & $90(6.3 \%)$ \\
\hline \multicolumn{2}{|c|}{ Faecal composition complaints } & $125(6.7 \%)$ & $126(6.8 \%)$ & $230(12.4 \%)$ \\
\hline \multicolumn{2}{|c|}{ Abdominal pain during defecation } & $99(5.3 \%)$ & $57(3.1 \%)$ & $90(4.8 \%)$ \\
\hline \multicolumn{2}{|c|}{ Anal pain during defecation } & $94(6.6 \%)$ & $63(4.4 \%)$ & $69(4.8 \%)$ \\
\hline \multicolumn{2}{|c|}{ Blood loss during defecation } & $36(2.5 \%)$ & $14(1.0 \%)$ & $10(0.7 \%)$ \\
\hline \multicolumn{2}{|c|}{ Visible prolapse during defecation } & $50(3.5 \%)$ & $16(1.1 \%)$ & $22(1.6 \%)$ \\
\hline \multicolumn{2}{|c|}{ Incomplete defecation } & $102(7.2 \%)$ & $104(8 \%)$ & $190(13.3 \%)$ \\
\hline
\end{tabular}




\begin{tabular}{|c|c|c|c|c|c|c|c|}
\hline & \multicolumn{2}{|c|}{ None/< once } & \multicolumn{2}{|c|}{ once } & $2-3$ times & 4-7 times & $>7$ times \\
\hline Frequency weekly urine loss & \multicolumn{2}{|c|}{$1198(64.4 \%)$} & \multicolumn{2}{|c|}{$32(1.7 \%)$} & $77(4.1 \%)$ & $187(10.0 \%)$ & $367(19.7 \%)$ \\
\hline \multirow[t]{2}{*}{ Frequency $24 \mathrm{hrs}$. urine loss } & \multicolumn{2}{|c|}{1219 (65.5\%) } & \multicolumn{2}{|c|}{$116(6.2 \%)$} & $267(14.3 \%)$ & $186(10.0 \%)$ & $73(3.9 \%)$ \\
\hline & \multicolumn{2}{|c|}{ drops } & \multicolumn{2}{|c|}{ dashes } & \multicolumn{2}{|c|}{ varies strongly } & cole load \\
\hline \multirow[t]{2}{*}{ Quantity of urine loss } & \multicolumn{2}{|c|}{$170(9.1 \%)$} & \multicolumn{2}{|c|}{$302(16.2 \%)$} & \multicolumn{2}{|c|}{$177(9.5 \%)$} & $(2.0 \%)$ \\
\hline & 1-3 months & \multicolumn{2}{|c|}{ 4-12 months } & \multicolumn{2}{|c|}{$13-24$ months } & $>2$ years & \\
\hline \multirow[t]{2}{*}{ Ul duration } & $4 \%)$ & \multicolumn{2}{|c|}{$170(9.1 \%)$} & \multicolumn{2}{|c|}{$42(2.2 \%)$} & $48(24.1 \%$ & \\
\hline & \multicolumn{2}{|c|}{$<6$} & & \multicolumn{2}{|c|}{$6-10$} & $>10$ & \\
\hline \multirow[t]{2}{*}{ Daytime urinary frequency } & \multicolumn{3}{|c|}{$808(43.5 \%)$} & \multicolumn{2}{|c|}{759 (40.9\%) } & \multicolumn{2}{|c|}{$253(13.7 \%)$} \\
\hline & \multicolumn{3}{|c|}{ Never } & \multicolumn{2}{|l|}{$1-3$} & $>3$ & \\
\hline \multicolumn{2}{|c|}{ Frequency of UTI past 12 months } & $308(70$. & & 291 & $(15.7 \%)$ & $253(13$ & $\%)$ \\
\hline
\end{tabular}

$\mathrm{UTI}=$ urinary tract infection.

Table 2. Statistical characteristics of prevalence in HADS anxiety and depression scales in patients contacted for the first time at the PCC during 2008-2013 ( $N=1862)$.

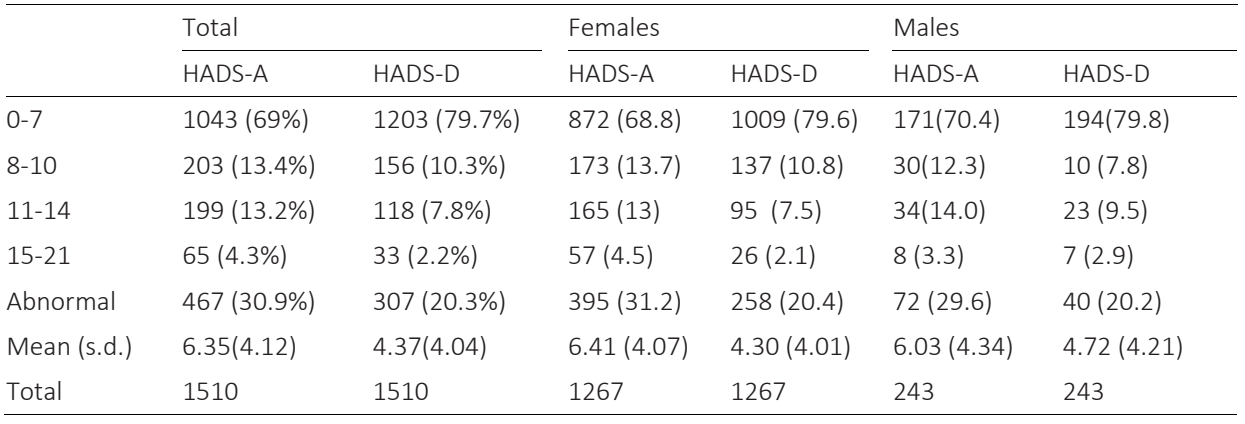

Frequencies (and percentages) of categorized HADS scores. 
Table 3. Final model results from dummy-regression analysis on square-root-transformed scores of HADS depression scale as a dependent variable using clinical predictors and background demographics. Variance explained: $0.120 . \mathrm{N}=1506$.

\begin{tabular}{|c|c|c|c|c|c|c|c|}
\hline \multirow[t]{2}{*}{ Predictors: } & \multicolumn{2}{|c|}{$\begin{array}{l}\text { Unstandardized } \\
\text { Coefficients }\end{array}$} & \multirow[t]{2}{*}{$\begin{array}{l}\text { Standardized } \\
\text { Coefficients }\end{array}$} & \multirow[b]{2}{*}{$\mathrm{t}$} & \multirow[b]{2}{*}{ p-value } & \multicolumn{2}{|c|}{$\begin{array}{l}95 \% \text { Confidence } \\
\text { Interval for B }\end{array}$} \\
\hline & $\mathrm{B}$ & $\begin{array}{l}\text { Std. } \\
\text { Error }\end{array}$ & & & & $\begin{array}{l}\text { Lower } \\
\text { Bound }\end{array}$ & $\begin{array}{l}\text { Upper } \\
\text { Bound }\end{array}$ \\
\hline (Constant) & 0.891 & 0.118 & & 7.580 & $<0.001$ & 0.660 & 10.121 \\
\hline Age & 0.008 & 0.002 & 0.127 & 4.979 & $<0.001$ & 0.005 & 0.011 \\
\hline Gender( $0=F, 1=M)$ & 0.363 & 0.125 & 0.127 & 2.894 & 0.004 & 0.117 & 0.609 \\
\hline Prevalence Urine Incontinence & 0.220 & 0.067 & 0.102 & 3.263 & 0.001 & 0.088 & 0.352 \\
\hline Prevalence Voiding problems & -0.021 & 0.067 & -0.010 & -0.306 & 0.760 & -0.152 & 0.111 \\
\hline Prevalence Prolapse problems & -0.322 & 0.091 & -0.138 & -3.549 & $<0.001$ & -0.500 & -0.144 \\
\hline Prevalence Obstipation & 0.122 & 0.090 & 0.036 & 1.353 & 0.176 & -0.055 & 0.300 \\
\hline Prevalence Faecal Incontinence & -0.001 & 0.086 & 0.000 & -0.007 & 0.994 & -0.169 & 0.168 \\
\hline Prevalence Sexual problems & 0.460 & 0.149 & 0.077 & 3.080 & 0.002 & 0.167 & 0.752 \\
\hline Complaints on faecal composition & 0.066 & 0.020 & 0.098 & 3.230 & 0.001 & 0.026 & 0.106 \\
\hline Having serious headaches & 0.156 & 0.044 & 0.089 & 3.525 & $<0.001$ & 0.069 & 0.243 \\
\hline Having bladder problems(past 6m) & 0.049 & 0.024 & 0.058 & 2.093 & 0.037 & 0.003 & 0.095 \\
\hline Freq. Urine Incontinence(24hrs) & 0.084 & 0.028 & 0.104 & 3.052 & 0.002 & 0.030 & 0.139 \\
\hline Having burning sensation & 0.056 & 0.022 & 0.065 & 2.541 & 0.011 & 0.013 & 0.099 \\
\hline Diabetes Mellitus & 0.101 & 0.047 & 0.054 & 2.131 & 0.033 & 0.008 & 0.193 \\
\hline Prolapse-related Voiding Problems & 0.284 & 0.118 & 0.099 & 2.413 & 0.016 & 0.053 & 0.515 \\
\hline Gender-related Faecal Incontinenc* & 0.366 & 0.188 & 0.057 & 1.950 & 0.051 & -0.002 & 0.735 \\
\hline Gender-related freq. UI.(24hrs)* & -0.145 & 0.055 & -0.111 & -2.608 & 0.009 & -0.254 & -0.036 \\
\hline
\end{tabular}

* Effects presented are for male patients. For female patients apply reverse sign on B, Beta or t coefficient.

Table 4 shows the results of the final (dummy) regression model for HADS-anxiety. Six direct regression effects and five first-order interaction effects have statistically significant effects upon HADS anxiety. Final results can be summarized as follows, interpreting the unstandardized regression coefficients. 1. (0.276) If patients responded affirmativeIy on having sexual problems, they showed higher scores on HADS anxiety sub-scores. 2. (0.135) If patients suffered from serious headaches, they had higher scores on HADS anxiety. 3. (0.053) If patients complained on bladder emptying during the past 6 months, they had higher scores on HADS anxiety. 4. (0.048) If patients said that they had a burning or pain while urinating in the past 6 months, they were likely to score high on HADS anxiety. 5. (0.211) If patients suffered from kidney stones, they also showed high scores on HADS anxiety. 6. (0.059) If patients complained about defecation during the past 6 months, they were likely to score high on HADS anxiety. 7. (0.208) If patients said that they had prolapse-related voiding problems, they had high scores on HADS anxiety sub-scores. 8. (0.006) Urine incontinence in senior patients had higher effects on HADS anxiety than urine incontinence in younger patients. 9. (0.371) The 
higher the obstipation problem, the higher the score is on HADS anxiety was, but this effect is significantly higher in female patients than in men. 10. (0.069) If patients reported faecal incontinence, they generally had higher HADS anxiety scores, which is significantly higher in men. 11. (-0.008) Older women showed higher scores in HADS anxiety than older men. The explained variance for the final regression model in 1507 patients is 0.074 .

Table 4. Final model results from dummy-regression analysis on square-root-transformed scores of HADS anxiety scale as a dependent variable using clinical predictors and background demographics. Variance explained: $0.074 \mathrm{~N}=1507$.

\begin{tabular}{|c|c|c|c|c|c|c|c|}
\hline \multirow[t]{2}{*}{ Predictors: } & \multicolumn{2}{|c|}{$\begin{array}{l}\text { Unstandardized } \\
\text { Coefficients }\end{array}$} & \multirow[t]{2}{*}{$\begin{array}{l}\text { Standardized } \\
\text { Coefficients }\end{array}$} & \multirow[b]{2}{*}{$\mathrm{t}$} & \multirow[b]{2}{*}{ p-value } & \multicolumn{2}{|c|}{$\begin{array}{l}\text { 95\% Confidence } \\
\text { Interval for B }\end{array}$} \\
\hline & B & $\begin{array}{l}\text { Std. } \\
\text { Error }\end{array}$ & & & & $\begin{array}{l}\text { Lower } \\
\text { Bound }\end{array}$ & $\begin{array}{l}\text { Upper } \\
\text { Bound }\end{array}$ \\
\hline (Constant) & 2.052 & 0.117 & & 17.605 & $<0.001$ & 10.823 & 20.280 \\
\hline Age & 0.002 & 0.002 & 0.044 & 1.308 & 0.191 & -0.001 & 0.006 \\
\hline Gender & 0.239 & 0.229 & 0.098 & 1.041 & 0.298 & -0.211 & 0.689 \\
\hline Prevalence Urine Incontinence & -0.244 & 0.179 & -0.133 & -1.365 & 0.173 & -0.595 & 0.107 \\
\hline Prevalence Voiding problems & -0.037 & 0.058 & -0.021 & -0.649 & 0.516 & -0.150 & 0.076 \\
\hline Prevalence Prolapse problems & -0.209 & 0.079 & -0.105 & -2.656 & 0.008 & -0.363 & -0.055 \\
\hline Prevalence Obstipation & 0.106 & 0.083 & 0.037 & 1.281 & 0.200 & -0.056 & 0.269 \\
\hline Prevalence Faecal Incontinence & 0.060 & 0.072 & 0.024 & 0.830 & 0.407 & -0.081 & 0.200 \\
\hline Prevalence Sexual problems & 0.276 & 0.131 & 0.054 & 2.115 & 0.035 & 0.020 & 0.533 \\
\hline Having serious headaches & 0.135 & 0.038 & 0.090 & 3.527 & $<0.001$ & 0.060 & 0.211 \\
\hline Having bladder problems(past $6 \mathrm{~m}$ ) & 0.053 & 0.020 & 0.073 & 2.663 & 0.008 & 0.014 & 0.091 \\
\hline Having Kidney stones & 0.211 & 0.073 & 0.073 & 2.889 & 0.004 & 0.068 & 0.354 \\
\hline Having burning sensation & 0.048 & 0.019 & 0.066 & 2.516 & 0.012 & 0.011 & 0.086 \\
\hline Having slime in faeces (past 6m) & 0.059 & 0.029 & 0.054 & 2.040 & 0.041 & 0.002 & 0.115 \\
\hline Prolapse-related Voiding Problems & 0.208 & 0.103 & 0.085 & 2.024 & 0.043 & 0.006 & 0.409 \\
\hline Urine Incontinence * Age & 0.006 & 0.003 & 0.219 & 2.185 & 0.029 & 0.001 & 0.012 \\
\hline Gender-related Obstipation* & 0.371 & 0.191 & 0.057 & 1.941 & 0.052 & -0.004 & 0.747 \\
\hline $\begin{array}{l}\text { Gender-related Faecal } \\
\text { Incontinence* }\end{array}$ & 0.377 & 0.167 & 0.069 & 2.261 & 0.024 & 0.050 & 0.704 \\
\hline Age * Gender* & -0.008 & 0.004 & -0.194 & -2.051 & 0.040 & -0.015 & 0.000 \\
\hline
\end{tabular}

*Effects presented are for male patients. For female patients apply reverse sign on B, Beta or t coefficient.

\section{DISCUSSION}

Although multidisciplinary care is advocated for PFDs ${ }^{17}$, up to now, psychological assessment is still not routinely incorporated into the assessment of PFDs. As in other multidisciplinary efforts ${ }^{18}$ our cohort data demonstrate functional complaints are strongly associated with emotion regulation in PFDs. At our PCC, 30\% of patients with PFDs had an 
abnormal anxiety score and $20 \%$ an abnormal depression score. Furthermore, corrected for confounding factors, PFDs may account for $7.4 \%$ of the variance in anxiety scores and $12 \%$ of the variance in depression scores. These findings are in accordance with the literature concerning psychiatric and somatic comorbidity in general and with regard to PFDs in particular. For example, in cancer patients, abnormal HADS anxiety scores were found in $30 \%$ of the patients and an abnormal depression scores were seen in $24.1 \%^{19}$. In a cohort of patients with chronic obstructive pulmonary disease (COPD) hospitalized for an acute exacerbation, the prevalence of an abnormal HADS depression score was $44 \%$ at admittance, and $11 \%$ had an abnormal HADS anxiety score ${ }^{20}$ The prevalence of affective disorders in patients with overactive bladder is considerable ${ }^{7}$. In the UK, HADS anxiety scores of 8 and higher were found in 30\% and $47 \%$ of men with OAB without and with bother respectively, and in women this was $44 \%$ for OAB without bother, up to almost $60 \%$ in $O A B$ with bother ${ }^{21}$. Prevalence rates in Sweden were similar to our results with aberrant scores of $8 \%-19 \%$ in men and $18 \%-32 \%$ in women for OAB without and with bother respectively ${ }^{21}$. Depression scores in our cohort are similar to scores reported by Swedish men and women with $O A B$, but lower compared to UK men and women ${ }^{21}$. Anxiety and depression scores, as measured by the HADS, were much higher for women with urgency urinary incontinence (UUI). The majority of women with UUI reported anxiety (56\%) and over a third reported depression ${ }^{22}$. The prevalence of depression and anxiety in patients with overall PFDs is not well studied. In the US, a prevalence of $22 \%$ depressed patients among women seeking treatment for advanced pelvic organ prolapse has been reported. This was five times higher in women with POP compared to those without ${ }^{23}$. Additionally, within the group of women with prolapse, women with symptoms of depression reported lower quality of life and worse prolapse related symptoms scores compared to women without depressive symptoms ${ }^{23}$. Moreover, a qualitative study regarding the emotional burden experienced by women seeking treatment for prolapse revealed that women's psychological well-being is closely related to their pelvic floor symptoms ${ }^{24}$. In our cohort, there are many patients with multifactorial causes of PFDs or interacting PFDs. Patients with urinary incontinence, especially in case of higher severity and complexity due to other health problems (e.g. headache or diabetes mellitus) showed higher HADS depression and anxiety scores. This is in line with the findings of Di Gangi Herms et al. who found that patients with low-complexity incontinence problems had fewer depressive or anxiety related symptoms, less distress, and better clinical outcome at follow-up, than patients with high-complexity ${ }^{25}$.

There may be some reluctance in the field to consider a broader multidisciplinary approach as laid down in the National Institute for Health and Care Excellence (NICE) urinary incontinence guideline, which may be related in part to low diagnostic sensitivity for comorbidity ${ }^{26}$. This is probably due to the practical difficulties related to the medical specialists experience in looking after patients with PFDs, since many will not have had sufficient training and will not schedule sufficient time and resources to implement such assessment. Nevertheless psychometric questionnaires can detect mental co-morbidity 
easily in somatic patients. The HADS performs well in the screening for depression or anxiety, and detects cases of anxiety disorders and depression in somatic, psychiatric and primary care patients and in the general population ${ }^{27}$. Psychiatric case detection is important, given that psychiatric disorders affect the prognosis of somatic conditions and the level of health care consumption in general ${ }^{28}$. Depression can adversely affect selfcare and may increase the risk of new medical complaints, complication and mortality ${ }^{28}$. Quite similar, anxiety can amplify symptoms of medical illnesses and may worsen clinical outcomes ${ }^{29}$. In a longitudinal study, depression was, at baseline, associated with the persistence of urinary incontinence (UI) in women ${ }^{30}$, and anxiety and depression may intensify UI and decrease the benefit of treatment ${ }^{31}$. A large population based survey revealed an inverse relationship between depressive symptoms and health care seeking. Depressive symptoms may thus prevent patients from seeking treatment ${ }^{21}$. It is possible that there is a reinforcing cycle of increasing PFD symptoms, with increasing bother and increasing affective complaints that does not lead to consecutive treatment seeking.

However, combined consultations of a psychiatrist and a uro-gynaecologist, urologist or colorectal surgeon may not be feasible in many practice situations. Nevertheless, our PCC team increasingly experienced that especially patients with complicated and multifactorial PFDs appeared to have (hidden) symptoms of depression and/or anxiety, which is clearly supported by different observations in literature. Moreover, psychiatric disorders affect the prognosis of somatic conditions and the level of health care consumption in general ${ }^{28}$, and the present findings demonstrate the importance of an interdisciplinary approach in patients with PFDs, addressing physical, social, emotional and relational aspects.

The results of our investigation should be seen in the light of limitations. Due to the cross-sectional design of our study, we were not able to investigate causality aspects. Thus, the question whether depression and anxiety are causing PFDs or PDFs are a cause of affective conditions remains unanswered. Hopefully, we are able to present a follow-up study of the effects of treatment of PFDs on the HADS score, and vice versa, in the near future.

\section{CONCLUSION}

In the present study anxiety and depression, suggestive for affective conditions, are prevalent (30.9\% and $20.3 \%$, respectively) in a cohort of patients with PFDs. In our final regression model, the explained variance of depression and anxiety, measured with the HADS was $12 \%$ and $7.4 \%$ respectively, meaning that, corrected for other contributing variables/factors, $12 \%$ of depression and $7.4 \%$ of anxiety was directly related to PFDs. Whether depression or anxiety may lead to PFDs was not part of this investigation. We advocate a multidisciplinary approach including psychological and psychiatric assessment, using the HADS questionnaire, at least in complex PFD patients. 


\section{REFERENCES}

1. Milsom, I., Altman, D, Cartwright, M.C., Epidemiology of urinary incontinence (IU) and other lower urinary tract symptoms (LUTS), pelvic organ prolapse (POP) and anal incontinence, in Incontinence, P. Abrams, Editor. 2013. p. 15-108.

2. Luber, K.M., S. Boero, and J.Y. Choe, The demographics of pelvic floor disorders: current observations and future projections. Am J Obstet Gynecol, 2001. 184(7): p. 1496-501; discussion 1501-3.

3. Davis, K.J., D. Kumar, and M.C. Wake, Pelvic floor dysfunction: a scoping study exploring current service provision in the UK, interprofessional collaboration and future management priorities. Int J Clin Pract, 2010. 64(12): p. 1661-70.

4. Berghmans, B., et al., Prevalence and triage of first-contact complaints on pelvic floor dysfunctions in female patients at a Pelvic Care Centre. Neurourol Urodyn, 2016. Apr;35(4): p. 503-8.

5. Coyne, K.S., et al., National community prevalence of overactive bladder in the United States stratified by sex and age. Urology, 2011. 77(5): p. 1081-7.

6. Abrams, P., et al., The standardisation of terminology in lower urinary tract function: report from the standardisation sub-committee of the International Continence Society. Urology, 2003. 61(1): p. 37-49.

7. Vrijens, D., et al., Affective symptoms and the overactive bladder-a systematic review. J Psychosom Res, 2015. 78(2): p. 95-108.

8. Melville, J.L., et al., Incontinence severity and major depression in incontinent women. Obstet Gynecol, 2005. 106(3): p. 585-92.

9. Leue, C., et al., Bidirectional associations between depression/anxiety and bowel disease in a population based cohort. J Epidemiol Community Health, 2005. 59(5): p. 434.

10. Regier, D.A., E.A. Kuhl, and D.J. Kupfer, The DSM-5: Classification and criteria changes. World Psychiatry, 2013. 12(2): p. 92-98.

11. Wang, P.S., et al., Twelve-month use of mental health services in the United States: results from the National Comorbidity Survey Replication. Arch Gen Psychiatry, 2005. 62(6): p. 629-40.

12. Meyer, T., H. Klemme, and C. Herrmann, Depression but not anxiety is a significant predictor of physicians' assessments of medical status in physically ill patients. Psychother Psychosom, 2000. 69(3): p. 147-54.

13. Fukudo, S., et al., Evidence-based clinical practice guidelines for irritable bowel syndrome. J Gastroenterol, 2015. 50(1): p. 11-30.

14. Fink, P., M.S. Hansen, and M.L. Oxhoj, The prevalence of somatoform disorders among internal medical inpatients. J Psychosom Res, 2004. 56(4): p. 413-8.

15. Leue, C., et al., Managing complex patients on a medical psychiatric unit: an observational study of university hospital costs associated with medical service use, length of stay, and psychiatric intervention. J Psychosom Res, 2010. 68(3): p. 295-302.

16. Zigmond, A.S. and R.P. Snaith, The hospital anxiety and depression scale. Acta Psychiatr Scand, 1983. 67(6): p. 361-70.

17. Chatoor, D., M. Soligo, and A. Emmanuel, Organising a clinical service for patients with pelvic floor disorders. Best Pract Res Clin Gastroenterol, 2009. 23(4): p. 611-20.

18. Clemens, J.Q., et al., The MAPP research network: a novel study of urologic chronic pelvic pain syndromes. Bmc Urology, 2014. 14;58.

19. Cardoso, G., et al., Depression and anxiety symptoms following cancer diagnosis: a cross-sectional study. Psychol Health Med, 2015: p. 1-9.

20. Ng, T.P., et al., Depressive symptoms and chronic obstructive pulmonary disease: effect on mortality, hospital readmission, symptom burden, functional status, and quality of life. Arch Intern Med, 2007. 167(1): p. 60-7.

21. Coyne, K.S., et al., The impact of overactive bladder on mental health, work productivity and healthrelated quality of life in the UK and Sweden: results from EpiLUTS. BJU Int, 2011. 108(9): p. 1459-71. 
22. Perry, S., C.W. McGrother, and K. Turner, An investigation of the relationship between anxiety and depression and urge incontinence in women: Development of a psychological model. British Journal of Health Psychology, 2006. 11(3): p. 463-482.

23. Ghetti, C., et al., Depressive symptoms in women seeking surgery for pelvic organ prolapse. Int Urogynecol J, 2010. 21(7): p. 855-60.

24. Ghetti, C., et al., The Emotional Burden of Pelvic Organ Prolapse in Women Seeking Treatment: A Qualitative Study. Female Pelvic Med Reconstr Surg, 2015. 21(6): p. 332-8.

25. Di Gangi Herms, A.M., et al., Assessing health care needs and clinical outcome with urological case complexity: a study using INTERMED. Psychosomatics, 2003. 44(3): p. 196-203.

26. Balachandran, A., A. Monga, and J. Duckett, Management of female urinary incontinence: A survey of urogynaecologists' view on the NICE guideline. Journal of Obstetrics and Gynaecology, 2016. 36(4): p. 487-491.

27. Bjelland, I., et al., The validity of the Hospital Anxiety and Depression Scale - An updated literature review. Journal of Psychosomatic Research, 2002. 52(2): p. 69-77.

28. Penninx, B.W., et al., Depression and cardiac mortality: results from a community-based longitudinal study. Arch Gen Psychiatry, 2001. 58(3): p. 221-7.

29. Roy-Byrne, P.P., et al., Anxiety disorders and comorbid medical illness. Gen Hosp Psychiatry, 2008. 30(3): p. 208-25.

30. Maserejian, N.N., et al., Treatment status and risk factors for incidence and persistence of urinary incontinence in women. Int Urogynecol J, 2014. 25(6): p. 775-82.

31. Bogner, H.R., et al., Anxiety disorders and disability secondary to urinary incontinence among adults over age 50. Int J Psychiatry Med, 2002. 32(2): p. 141-54. 



\section{Chapter}

\section{The relationship between self-consciousness/awareness and bladder sensations -}

a comparative study of patients with overactive bladder and healthy volunteers

Vrijens DMJ, Marcelissen TAT, Drossaerts JM, Heeringa R, Degaillier SD, Leue C, van Koeveringe $\mathrm{GA}$.

Accepted Low Urin Tract Symptoms 


\section{ABSTRACT}

\section{Objectives}

To explore differences in bladder sensations between OAB patients and healthy volunteers by evaluating self-consciousness, self-awareness and affective complaints.

\section{Methods:}

A prospective, observational study was performed comparing patients with overactive bladder symptoms and healthy volunteers. During three days subjects filled out sensation related bladder diaries (SR-BD), self-consciousness questionnaires (SCS), selfawareness questionnaire (SSAS) and Hospital Anxiety and Depression scales (HADS). The SSAS was filled out at the second void of the first day.

\section{Results}

In total, 134 participants were included (66 volunteers and 68 patients). Patients had lower voided volumes (193 ml vs. $270 \mathrm{ml}, \mathrm{p}<0.05)$, higher urinary frequency (10.6 vs. $6.6, p<0.05)$ and higher urgency scores (2.0 vs. $1.2,(p<0.05)$ than volunteers, while perceived bladder fullness was similar. The self-consciousness scores were similar, but the self-awareness was significantly higher for patients (53.6 vs. 44.6, $p<0.05$ ). Patients had significantly higher scores for anxiety and depression according to the HADS.

\section{Conclusions}

There are significant differences in SR-BD between volunteers and patients with $O A B$. Patients had significantly higher self-awareness than volunteers, indicating that patients with $O A B$ may attribute different values to bodily signals. Future research is required to elaborate our knowledge on the perceived sensations and labelling of emotions in OAB. 


\section{INTRODUCTION}

In a normal situation voiding is usually initiated at a suitable time and place without constantly thinking about it. This process becomes different in patients with overactive bladder. Overactive bladder (OAB) is defined by the International Continence Society (ICS) as a symptom complex of urgency, usually accompanied by frequency and nocturia with (OAB wet) or without (OAB dry) urgency urinary incontinence (UUI) ${ }^{1}$. The cornerstone in the diagnosis of $O A B$ is urgency: 'sensations of a sudden compelling desire to void that are difficult to defer' ${ }^{1}$ referring to a pathological all-or nothing sensation that is different from the sensation of bladder fullness. In healthy volunteers normal bladder filling is characterised by a gradual increase in urge which has been described as first sensation of bladder filling-, and normal desire to void up to strong desire to void ${ }^{2}$. The pattern of urge is changed to urgency in $O A B$ patients. Pathological bladder sensations do not solely arise from uncoordinated detrusor contractions, since $56 \%$ of women with OAB dry do not have detrusor overactivity (DO) during urodynamics ${ }^{3}$. Additionally, only half of the patients with DO can feel detrusor contractions. The reason why patients with OAB experience urgency is not well understood. It has been suggested that patients experience bladder sensations in a different way than healthy individuals. Heeringa et al. showed that bladder sensations during physiological filling develop significantly different in $O A B$ patients compared to volunteers ${ }^{4}$. Various mechanisms could explain this difference, for example: are patients with $O A B$ more self-conscious and more aware of bodily sensations which could result in an exaggerated perception of urge? And are bladder sensations influenced by psychological factors which could trigger an emotional response? In this study, we tried to answer these questions by examining the differences in selfconsciousness, self-awareness and affective complaints in relation to bladder sensations.

\section{METHODS}

We conducted a prospective, observational, study comparing patients with overactive bladder symptoms and healthy volunteers.

\section{Inclusion criteria}

Patients (men and women) older than 18 years were included and formed the first group, they were diagnosed with $O A B$ by their urologist, using the criteria of: more than 8 micturitions per day on three consecutive days with at least one episode of urgency: (i.e. sudden compelling desire to void which cannot be postponed). The second group consisted of healthy volunteers (men and women) older than 18 years. Patients and healthy volunteers had to be well versed in Dutch. Patients were recruited at the outpatient clinic. Volunteers were recruited through advertisements and posters. 


\section{Exclusion criteria}

Subjects with a post-void residual urine volume of $>100 \mathrm{cc}$ determined by ultrasound and subjects with presence of a urinary tract infection which was determined by urine sticks, were excluded. Volunteers with current urinary complaints or a urologic history, determined by internet based questions, were also barred from the study.

\section{Study protocol}

The study protocol was approved by the local ethics committee (METC 09-2-095)

Following enrolment, $\mathrm{OAB}$ patients and volunteers were subjected to the same study protocol. At baseline subjects were evaluated with a dipstick urine test, to exclude a urinary tract infection and a post-void ultrasound to measure residual urine. If applicable, antimuscarinics were stopped 21 days prior to start of the study. In order to evaluate the bladder sensations, all study subjects were asked to fill out sensation-related bladder diaries (SR-BD), internet based. A SR-BD can evaluate bladder sensation in a non-invasive way at each void during normal daily life ${ }^{5}$. The bladder diaries contained a Visual Analogue Scale (VAS) to draw the perceived bladder sensation, ranging from empty bladder to full bladder and a 4-point scale to grade perception of urge/urgency ${ }^{5}$. The meaning of the 4 grades is: $0=$ no bladder sensation, $1=$ voiding can be delayed for at least 30 minutes, 2=voiding cannot be delayed more than 10 minutes, $3=$ voiding cannot be delayed. Additionally, all subjects filled out a self-consciousness questionnaire (SCS) and the Hospital Anxiety and Depression Scale (HADS) questionnaire at baseline. The Situational Self-Awareness Scale (SSAS) was asked to be filled out before the second void of the first day. This void was chosen because the first morning void is mostly done directly after awakening and people tend to do this habitually.

\section{Questionnaires}

The SCS (Table 1) contains three subscales measuring the general traits of private selfconsciousness (10 items), public self-consciousness (7 items), and social anxiety (6 items). It is scored on 5-point scales (scoring range: $0-4)$ and was validated for use in the Netherlands ${ }^{6}$. Higher scores indicate a higher self-consciousness. Cronbach's alpha for the total scale in the Dutch validation sample was $0.81^{6}$.

The SSAS (Table 1) was developed to quantify levels of public and private selfawareness ${ }^{7}$. It consists of questions on VAS scales, with anchors indicating 'Not at all' and 'Very intense feeling'. For this study we added bladder-specific questions (SSASbladder), such as: "I was aware of my bladder feelings" or "I was engaged with my bladder feelings". This part of the questionnaire is not validated. The validated SSAS is more sensitive to situational changes than the $\mathrm{SCS}^{7}$. Self-focus, whether public or private, can be dispositional or situational. Dispositional self-focus is often referred to as "self- 
consciousness," whereas situational self-focus is labelled "self-awareness" 6 . Higher scores indicate higher self-awareness and higher self-consciousness levels.

Table 1. The items of the Self-Consciousness Scale (SCS), ordered according to subscale structure (above) and the items of the Situational Self Awareness Scale (SSAS) (below).

Private Self-consciousness

I'm always trying to figure myself out.

Generally, I'm not very aware of myself.

I reflect about myself a lot.

I'm often the subject of my own fantasies.

I never scrutinize myself

I'm generally attentive to my inner feelings.

I'm constantly examining my motives.

I sometimes have the feeling that I'm off somewhere watching myself.

I'm alert to changes in my mood.

I'm aware of the way my mind works when work through a problem.

Public Self-consciousness

I'm concerned about my style of doing things.

I'm concerned about the way I present myself.

I'm self-consciousness about the way I look.

I usually worry about making a good impression.

One of the last things I do before I leave my house is look in the mirror.

I'm concerned about what other people think of me.

I'm usually aware of my appearance.

Social Anxiety

It takes me time to overcome my shyness a new situations

I have trouble working when someone is watching me.

I get embarrassed very easily.

I don't find it hard to talk to strangers.

I feel anxious when I speak in front of a group.

Large groups make me nervous.

Situational Self-Awareness Scale

1. Right now, I am keenly aware of everything in my environment.

2. Right now, I am conscious of my inner feelings.

3. Right now, I am concerned about the way I present myself.

4. Right now, I am self-conscious about the way I look.

5. Right now, I am conscious of what is going on around me.

6. Right now, I am reflective about my life.

7. Right now, I am concerned about what other people think of me.

8. Right now, I am aware of my innermost thoughts.

9. Right now, I am conscious of all objects around me.

The HADS is a short, self-assessment scale to screen for the presence of affective symptoms in medically ill patients. The 14 items are divided into two subscales that assess depression (HADS-D) and anxiety (HADS-A). The items are rated on 4-point Likert type scales $^{8}$. A HADS score of $\geq 8$ per subscale is considered abnormal ${ }^{8}$. 


\section{Statistical analysis}

Student's t-test (two-tailed) was used to compare means of continuous variables between the patients and volunteers and Mann-Whitney test for ordinal variables.

Bivariate correlation tools (Pearson) were used to assess the correlation between continuous variables, such as questionnaires and voiding characteristics.

Statistical significance was set at $p<0.05$. Analysis was performed using SPSS v23 software (IBM SPSS Statistics, USA).

\section{RESULTS}

Overall, 134 study participants were included; 66 volunteers and 68 patients. All patients and volunteers completed the three days, except for one volunteer who only completed one day.

There were differences in baseline characteristics between patients and volunteers, as the patients were older (60 vs. 48 years old) and the patients group consisted of more males (45 versus 31) compared to the volunteers (Table 2). Furthermore, patients voided significantly more often $(p<0.05)$ and with a lower volume per void $(p<0.05)$ compared to the volunteers (Table 3 ). The mean intake per day was comparable between patients (1904cc) and volunteers (1935cc).

Table 2. Baseline characteristics (N)

\begin{tabular}{llll}
\hline & Criterion & Patients & Volunteers \\
\hline Demographic & Number of participants & 68 & 66 \\
Male sex & 45 & 31 \\
Age (mean), years & 60 & 48 \\
& Antihypertensive & 22 & 5 \\
Diuretic & 4 & 0 \\
Alpha-blocker & 2 & 1 \\
5-alpha reductase inhibitor & 1 & 0 \\
Antimuscarinics & 6 & 0 \\
Anti-depressive & 2 & 1 \\
Diabetes medication & 8 & 1 \\
Prostate operation & 6 & 0 \\
Incontinence operation & 1 & 0 \\
Additional urologic history & 6 & 0 \\
Neurologic history & 2 & 0 \\
Gynaecologic history & 10 & 2 \\
Surgical history & 21 & 3 \\
\hline
\end{tabular}


Table 3. Voiding characteristics of the 3 day SR-BD in patients and volunteers

\begin{tabular}{llll}
\hline Variables & Patients & Volunteers & $P$ \\
\hline Mean voided volume per void, ml & 193 & 270 & $<0.05^{*}$ \\
Mean frequency per day, $\mathrm{n}$ & 10.6 & 6.6 & $<0.05^{*}$ \\
Urinary frequency per litre diuresis & 5.8 & 4.1 & $<0.05^{*}$ \\
Mean bladder fullness perception, VAS 0-100 & 66.1 & 60.4 & 0.051 \\
Mean urgency score, 0-3 & 2.0 & 1.2 & $<0.05^{*}$ \\
\hline
\end{tabular}

Legend: *=Statistical difference (independent T-test)

VAS=Visual Analogue Scale.

\section{Second void of the first day.}

This particular void was analysed more specifically with regard to self-awareness assessment. There was a significant difference $(p<0.05)$ in urgency for the second void of the first day between patients and volunteers. Furthermore, there were considerably more voids with urgency grade 3 and 4 in the patients (Figure 1). Patients had a significantly lower voided volume compared to the volunteers $(p<0.05)$.

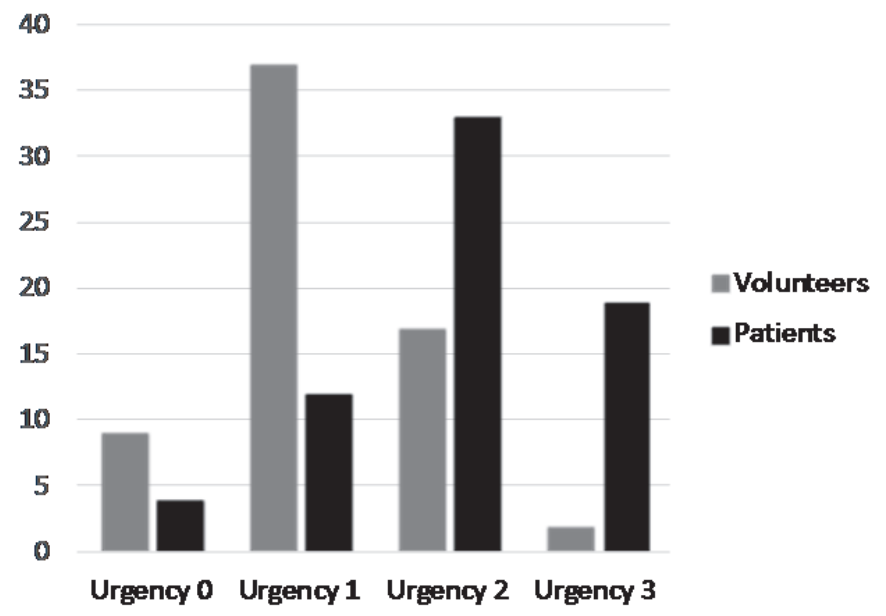

Figure 1. Number of voids per urgency score in volunteers and patients for the second void of the first day Urgency $0=$ no bladder sensation, Urgency $1=$ voiding can be delayed for at least 30 minutes, Urgency 2=voiding cannot be delayed more than 10 minutes, Urgency $3=$ voiding cannot be delayed.

\section{Self-consciousness and self-awareness scales.}

The self-consciousness scale and its subscales were similar for patients and volunteers.

The self-awareness scores for the second void of the first day was significantly higher in patients compared to volunteers $(p=0.003)$ (Table 4$)$. 
Chapter 4

Table 4. Self-consciousness and self-awareness scales.

\begin{tabular}{llll}
\hline Variables & Patients & Volunteers & $P$ \\
\hline SCS Total & 39.9 & 41.5 & 0.555 \\
SCS Social & 8.6 & 9.3 & 0.471 \\
SCS Public & 13.3 & 13.1 & 0.830 \\
SCS Private & 18.0 & 18.3 & 0.863 \\
SSAS total & 53.6 & 44.6 & $<0.05^{*}$ \\
SSAS bladder & 26.0 & 18.8 & $<0.05^{*}$ \\
\hline
\end{tabular}

Legend:* =statistical difference (independent T-test)

SCS Self-consciousness Scale, SSAS Situational Self-Awareness Scale.

HADS

Patients had significantly higher scores for anxiety and depression, but within the normal range (Table 5). Thirteen patients and 3 volunteers had an abnormal HADS score. The HADS score was significantly correlated with the SCS (Pearson correlation 0.327, $p=0.001$ ) and the SSAS (Pearson correlation 0.205, $p=0.018$ ).

Table 5. The screening results of patients and volunteers and the statistical difference between the groups.

\begin{tabular}{llll}
\hline Variables & Patients $(\mathrm{n}=68)$ & Volunteers $(\mathrm{n}=66)$ & $\mathrm{P}$ \\
\hline HADS-A (range) & $4.5(0-13)$ & $3.3(0-8)$ & $<0.05^{*}$ \\
HADS-D (range) & $3(0-11)$ & $1.2(0-5)$ & $<0.05^{*}$ \\
HADS total (range) & $7.5(0-24)$ & $4.5(0-13)$ & $<0.05^{*}$ \\
\hline
\end{tabular}

Legend ${ }^{*}=$ Statistical difference (independent T- test)

\section{DISCUSSION}

The present study is the first to comparatively investigate self-awareness and selfconsciousness, related to voiding in healthy volunteers and patients with OAB. The selfawareness scores were higher in patients compared to volunteers. At the time of filling out the SSAS, the voided volume was smaller in patients, but the urgency score was higher compared to the volunteers, while the bladder fullness sensation was similar in the two groups. This could imply that patients void more frequently due to lowered volume threshold for sensation compared to the volunteers.

Self-awareness is higher for patients, possibly pointing out that patients with $O A B$ are more sensitive to notice bodily sensations. Nevertheless no difference was found regarding self-consciousness between the patients and the volunteers. How can the difference in self-awareness, without a difference in self- consciousness, be explained? It has been described that the SSAS, which measures self-awareness is more sensitive to situational changes than the SCS, which measures self-consciousness ${ }^{7}$. Self-focus, 
whether public or private, can be dispositional or situational. Dispositional self-focus is often referred to as "self-consciousness," whereas situational self-focus is labelled "self-awareness" 6 . Thus, a comparable self-consciousness can be present between patients and volunteers, while the situational awareness may differ.

Functional MRI studies suggest that the insula mediates attention to interoceptive awareness ${ }^{9}$. Furthermore, the insula is also regarded as the cortex that registers and maps visceral sensations ${ }^{10}$. An influential review concerning awareness stated that "the anterior insula cortex provides a neural substrate that instantiates all subjective feelings from the body and feelings of emotion in the immediate present (now)" ${ }^{11}$. Local grey matter volume in the insula and its function can have variation between people and it has been described to correlate with both interoceptive accuracy and subjective ratings of visceral awareness in functional MRI studies ${ }^{9}$.

With regard to the bladder, the insula also plays an important role in the sensation of urgency. Patients with OAB showed increased activity during increased urgency in the insula and the Anterior Cingulate Cortex ${ }^{12}$. In addition, increased activation in insula and cingulate gyrus was observed as a response to greater degrees of bladder distension in subjects with poor bladder control ${ }^{13}$. Interestingly, it has been shown that not only the cerebral response to bladder filling is exaggerated in $O A B$, but that the whole response pattern is disturbed ${ }^{14}$. Similar activation patterns were observed in patients with irritable bowel syndrome (IBS), a disorder of intestinal hypersensitivity and altered motility ${ }^{15}$.

There is interpersonal variation in sensitivity to internal bodily responses, as seen in people with anxiety that have increased attention to autonomous bodily processes ${ }^{16}$. In the present study, the mean anxiety and depression scores were within the normal range, but 13 patients compared to 3 volunteers presented abnormal scores. The scores were significantly higher in patients with overactive bladder, which is in line with reports in the literature ${ }^{17}$. In addition, higher HADS scores were associated with higher self- consciousness and self-awareness. Hence, there is a possibility that patients with overactive bladder and anxiety and/or low mood states are more sensitive to bladder sensations, analogous to IBS in which enhanced visceral sensitivity plays a role in the pathophysiology ${ }^{18}$. A common bladder hypersensitivity syndrome is suggested because of the overlap between bladder pain syndrome (BPS) and OAB, both being at other ends of the same spectrum ${ }^{19}$. In other functional disorders a common hypersensitivity is suggested as well, with alarm falsification of normal bodily signals ${ }^{20}$.

Since psychological factors may contribute to symptom perception in $O A B$, different treatment strategies might be considered, as the current treatments of $O A B$ are not always successful ${ }^{21}$. These different strategies may either be part of psychopharmacological treatment, psychotherapy or brain stimulation. An interesting observation was done in women with predominantly UUI who were enrolled in an eight week Mindfulness-based stress reduction (MBSR) programme which significantly reduced the number of incontinence episodes. This reduction was still seen at twelve months follow up ${ }^{22}$. 
Mindful means paying attention on purpose, in the present moment and nonjudgmentally. The explanation of the authors of the working mechanism of MBSR was literally: "to teach participants skills that promote non-judgmental awareness of body sensations, to interpret body sensations as simply sensations as opposed to interpretations and thoughts about the sensations (e.g. catastrophizing). Mindfulness skills may allow participants to actually change how their brain responds to urgency by interpreting the sensation differently." Mindfulness based therapies have been proven to be beneficial in IBS ${ }^{18}$, part of which may be related to brain adaptations ${ }^{23}$.

Our study has several limitations. One limitation is the relatively small sample size. A second limitation is the differences in sex and age between volunteers and patients, indicating that both populations are not perfectly matched. The group of patients were older and consisted of more males.

Another limitation is the use of the questionnaires, the SCS and SSAS and the addition of non-validated bladder questions. The SCS and later revised forms are still widely used ${ }^{24}$ and it is the most popular self-consciousness instrument used in psychological research ${ }^{25}$. However, to our knowledge, both questionnaires have never been used or validated for the assessment of bladder sensations or OAB. Nevertheless, according to the results of our study, we think that these questionnaires may be useful in this context. We only used the SCS and SSAS at two time-points in our study, which could have influenced our results. This might be overcome in the future, by using Experience Sampling Methodology (ESM), in which random, repeated momentary assessments are recorded during the day. This digital research method asks subjects to stop at certain times and make notes of their experience in real time by asking specific questions, usually done through a mobile app. Hence, ESM may indicate potential psychological and emotional triggers that may influence symptom development and may lead to a better understanding of the nature of urgency and $O A B$. We are currently conducting a study with $\mathrm{ESM}$ in $\mathrm{OAB}$ patients.

The results of this observational study revealed a positive correlation between selfawareness and overactive bladder. It may be hypothesised that patients with overactive bladder respond differently to bladder sensations and are more sensitive to bodily signals. Future research is required to elaborate our knowledge on the perceived sensations and labelling of emotions in overactive bladder. 


\section{REFERENCES}

1. Abrams, P., et al., The standardisation of terminology of lower urinary tract function: report from the Standardisation Sub-committee of the International Continence Society. Neurourol Urodyn, 2002. 21(2): p. 167-78.

2. Wyndaele, J.J. and S. De Wachter, Cystometrical sensory data from a normal population: comparison of two groups of young healthy volunteers examined with 5 years interval. Eur Urol, 2002. 42(1): p. 34-8.

3. Hashim, H. and P. Abrams, Is the bladder a reliable witness for predicting detrusor overactivity? J Urol, 2006. 175(1): p. 191-4; discussion 194-5.

4. De Wachter, S.G., et al., "Focused introspection" during naturally increased diuresis: description and repeatability of a method to study bladder sensation non-invasively. Neurourol Urodyn, 2014. 33(5): p. 502-6.

5. De Wachter, S. and J.J. Wyndaele, Frequency-volume charts: a tool to evaluate bladder sensation. Neurourol Urodyn, 2003. 22(7): p. 638-42.

6. Vleeming, R.G. and J.A. Engelse, Assessment of private and public self-consciousness: a Dutch replication. J Pers Assess, 1981. 45(4): p. 385-9.

7. Govern, J.M. and L.A. Marsch, Development and validation of the situational self-awareness scale. Conscious Cogn, 2001. 10(3): p. 366-78.

8. Zigmond, A.S. and R.P. Snaith, The hospital anxiety and depression scale. Acta Psychiatr Scand, 1983. 67(6): p. 361-70.

9. Critchley, H.D., et al., Neural systems supporting interoceptive awareness. Nat Neurosci, 2004. 7(2): p. 189-95.

10. Kuhtz-Buschbeck, J.P., et al., Cortical representation of the urge to void: a functional magnetic resonance imaging study. J Urol, 2005. 174(4 Pt 1): p. 1477-81.

11. Craig, A.D., How do you feel--now? The anterior insula and human awareness. Nat Rev Neurosci, 2009. 10(1): p. 59-70.

12. Komesu, Y.M., et al., Functional MRI of the Brain in Women with Overactive Bladder: Brain Activation During Urinary Urgency. Female Pelvic medicine and reconstructive surgery, 2011. 30(1542-5983 (Electronic)): p. 3.

13. Griffiths, D., et al., Brain control of normal and overactive bladder. J Urol, 2005. 174(5): p. 1862-7.

14. Griffiths, D., Imaging bladder sensations. Neurourol Urodyn, 2007. 26(6 Suppl): p. 899-903.

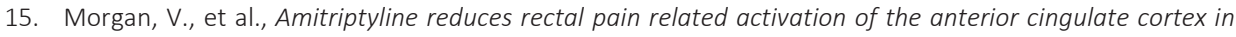
patients with irritable bowel syndrome. Gut, 2005. 54(5): p. 601-7.

16. Mumford, D.B., et al., The Bradford Somatic Inventory. A multi-ethnic inventory of somatic symptoms reported by anxious and depressed patients in Britain and the Indo-Pakistan subcontinent. Br J Psychiatry, 1991. 158: p. 379-86.

17. Vrijens, D., et al., Affective symptoms and the overactive bladder - A systematic review. Journal of Psychosomatic Research 2015;78:95-108

18. Aucoin, M., M.J. Lalonde-Parsi, and K. Cooley, Mindfulness-based therapies in the treatment of functional gastrointestinal disorders: a meta-analysis. Evid Based Complement Alternat Med, 2014. 2014: p. 140724.

19. Lai, H.H., et al., The overlap and distinction of self-reported symptoms between interstitial cystitis/bladder pain syndrome and overactive bladder: a questionnaire based analysis. J Urol, 2014. 192(6): p. 1679-85.

20. Fink, P. and A. Schroder, One single diagnosis, bodily distress syndrome, succeeded to capture 10 diagnostic categories of functional somatic syndromes and somatoform disorders. J Psychosom Res, 2010. 68(5): p. 415-26.

21. Burkhard, F.C., Lucas, M.G. , Berghmans, J.L. H.R. , Cruz, F., Lemack, G.E., Nambiar, A.K., Nilsson, C.G., Pickard, R. Tubaro, A. , EAU Guidelines on urinary incontinence in adults. 2016. 


\section{Chapter 4}

22. Baker, J., et al., Comparison of mindfulness-based stress reduction versus yoga on urinary urge incontinence: a randomized pilot study. with 6-month and 1-year follow-up visits. Female Pelvic Med Reconstr Surg, 2014. 20(3): p. 141-6.

23. Lutz, J., et al., Altered processing of self-related emotional stimuli in mindfulness meditators. Neuroimage, 2016. 124(Pt A): p. 958-67.

24. DaSilveira, A., M.L. DeSouza, and W.B. Gomes, Self-consciousness concept and assessment in self-report measures. Front Psychol, 2015. 6: p. 930.

25. Cramer, K.M., Comparing the relative fit of various factor models of the self-consciousness scale in two independent samples. J Pers Assess, 2000. 75(2): p. 295-307. 



\section{Chapter}

\section{Associations of psychometric affective parameters with urodynamic investigation for urinary frequency.}

Vrijens DMJ, Drossaerts JM, Rademakers K, Smits MA, De Wachter SG, Leue C, van Koeveringe $G A$.

Low Urin Tract Symptoms 2016 Jun 12, (Epub ahead of print) 


\section{ABSTRACT}

\section{Objectives}

To assess an association between affective symptoms and conventional urodynamic results in a pilot study.

\section{Methods}

The study represents a retrospective analysis of prospectively obtained clinical data, voiding diaries, urodynamic parameters and Hospital Anxiety and Depression Scale (HADS).

\section{Results:}

A total of 74 patients with urinary frequency attending a multidisciplinary Pelvic Care Centre were included in this study. There was a significant association between the total HADS scores and presence of detrusor overactivity (DO) ( $p=0.019)$. In addition, results showed an association between HADS anxiety scores $(\geq 8)$ and DO $(p=0.018)$ and between HADS depression scores $(\geq 8)$ and the feeling of urgency $(p=0.028)$. Comparative analysis showed differences in age, mean voiding volume, bladder capacity and strong desire between patients with DO and those without.

\section{Conclusion:}

This pilot study revealed an association between psychometric parameters and urodynamic results indicating a common pathway of bladder function and affective complaints. Further research is needed to elucidate which parts of the bladder-brain axis are involved and how these parts correspond by means of urodynamics. 


\section{INTRODUCTION}

Depression and anxiety disorders are diagnosed according to the DSM-V criteria ${ }^{1}$. Affective conditions have a relatively high lifetime prevalence ranging from $2-18 \%{ }^{2,3}$. As early as 1964, the relationship between depression and anxiety and lower urinary tract symptoms (LUTS) has been described ${ }^{4}$. Almost 20 years later the importance of anxiety and depression in the aetiology of urgency incontinence and overactive bladder syndrome (OAB) was emphasized ${ }^{5}$. OAB is defined by the International Continence Society (ICS) as a symptom complex of urgency, usually accompanied by frequency and nocturia, with or without urinary urgency incontinence ${ }^{6}$. Urgency is the key symptom in $O A B$ but frequency is considered one of the most bothersome symptoms of $O A B{ }^{7}$. It is a common condition with approximately $16-17 \%$ of the adult population affected ${ }^{8}$. OAB is a diagnosis based on patients' symptoms. In contrast, detrusor overactivity (DO) is defined as involuntary detrusor contractions, during the filling phase of conventional urodynamic study (conventional-UDS) ${ }^{9}$. OAB patients with DO have smaller maximum voided volumes per void and experience significantly more incontinent episodes. Additionally, OAB patients with DO are more likely to have abnormal sensations, with a strong desire and urgency occurring at significantly lower bladder volumes during UDS compared to those without DO. There are two groups of patients: OAB with DO and without DO ${ }^{10}$. Recently, the growing interest concerning the association of affective symptoms with $O A B$ as a possible interface of stress related pathophysiology in $O A B$, is summarized in a systematic review. This review, consisting of 43 articles and 80.000 subjects, revealed that there is an association between $O A B$ and affective symptoms in almost all studies ${ }^{11}$. Although there have been numerous articles about OAB and affective symptoms, studies combining UDS and affective symptoms in order to elucidate pathophysiological interaction are sparse. The objective of this study is to assess the association between voiding diaries, different urodynamic parameters, (such as DO, bladder capacity and sensation markers) and affective symptoms in a University Hospital cohort of patients with frequency.

\section{METHODS}

This retrospective analysis of prospectively collected clinical data during a period of one year was conducted in patients who attended the Pelvic Care Centre (PCC) Maastricht. This is a multidisciplinary pelvic care clinic, characterized by integrated care delivered by urologists, gynaecologists, procto-rectal surgeons, physical therapists, sexologists and psychiatrists. Patients with bladder complaints and a voiding frequency of $\geq 7$ times per 24 hours, as described in the database, were included if: a) complete charts were available for revision, b) completed bladder diaries during 3 days were present and c) Hospital Anxiety and Depression Scale (HADS) prior to d) urodynamic investigation was com- 
pleted. The HADS is a validated self-assessment scale for the detection of anxiety or depression symptoms in a non-psychiatric (hospital) outpatient population. It consists of a seven anxiety domain questions (HADS-A) and seven questions on the depression domain (HADS-D) ${ }^{12}$. Scores of $\geq 8$ per domain indicate an increased risk for anxiety or depression. Voiding diaries provide information about fluid intake, number and volume of voidings, feelings of urgency and bladder fullness, and possible loss of urine.

The urodynamic measurements were performed with use of 'Medical Measurements Systems' (MMS B.V., Enschede, The Netherlands) equipment and according to International Continence Society (ICS) standards ${ }^{13}$. Patients discontinued anticholinergic medication at least five days before the urodynamic assessment. Patients' urine was cultured ten days before conventional-UDS. In case of bacteriuria, focused antibiotic treatment was provided after which recovery of the bacteriuria was confirmed before the investigation was executed.

HADS scores, voiding diaries and urodynamic investigations were retrevied from patient records, including complaints (self reported incontinence), medical and psychatric history and medication.

The urodynamic investigations were evaluated using a standardised protocol by three urologists (GVK, SdW, and DV) who are specialised in functional urology. The urologists were blinded for the complaints and patient characteristics in order to reduce bias. During filling cystometry, the volume of first sensation, normal and strong desire to void was annotated as well as several other parameters: the total bladder capacity, the presence of DO and the presence of incontinence (SUI, UUI, UDS proven incontinence). DO is defined as rise in the detrusor pressure during the filling phase of conventional-UDS.

\section{Statistical analysis}

Pearson $\chi^{2}$ tests were used to calculate associations; T-tests for comparisons in SPSS (version 20 / 22 IBM etc.). P-values $<0.05$ were considered as statistically significant. Confidence intervals (CI) of 95\% were calculated. The HADS-D and HADS-A scales were dichotomized with a cut-off point of 8 (normal vs. abnormal). Additionally, the total HADS score was used to calculate associations with a cut-off point of 15 (normal vs. abnormal). Simple linear regression was used for the calculation of the association between intake and frequency.

\section{RESULTS}

A total of 74 patients were included with frequency (Figure 1). Forty-three of these patients (58.1\%) reported urgency and can be considered as typical OAB patients. Incontinence was reported by $72 \%$; type and severity of incontinence were not reported. The majority $(73 \%)$ of the patients was female, with a mean age of 56.7 years. Twenty six of 
the 54 women had gynaecological surgery in the past. Psychiatric complaints, mostly depression, were present in 19 patients (25\%; 22\% depression, 3\% anxiety) (Table 1). The mean daily urine frequency was 11.4 times (s.d. 3.4) with an average of 1.4 (s.d. 1.3) episodes of nocturia recorded on the voiding diary. Mean total urine production was $2019 \mathrm{ml}$ per 24 hours (s.d. $836 \mathrm{ml}$ ). Frequency and total volume of urine production per 24 hours was not associated (Linear regression, $A N O V A, R=0.211, R^{2}=0.044, p=0.071$ ). DO was present in 32 patients (43\%). Urgency urinary incontinence (UUI) was observed in 17 patients (23\%), stress urinary incontinence (SUI) in 11 patients (14\%) and 1 patient had mixed incontinence. The overall UDS-diagnosis was no OAB in 41 patients, OAB wet in 15 patients and $\mathrm{OAB}$ dry in 18 patients. The voiding phase was normal in 33 patients, obstructive voiding was seen in 9 patients and dysfunctional voiding in 32 patients.

Table 1. Patients characteristics and previous history, patients with detrusor overactivity (DO) and without.

\begin{tabular}{lll}
\hline & Urinary Frequency with DO & Urinary Frequency without DO \\
\hline Number of patients & 32 & 42 \\
Age (years) & 59 & 53 \\
Females (n) & 16 & 38 \\
Urinary tract infection (n) & 5 & 14 \\
Incontinence surgery (n) & 1 & 2 \\
Benign prostate hyperplasia (n) & 1 & 0 \\
Anxiety (n) & 0 & 2 \\
Depression (n) & 7 & 9 \\
Diabetes Mellitus $(\mathrm{n})$ & 3 & 4 \\
Abdominal surgery $(\mathrm{n})$ & 6 & 9 \\
Gynaecological surgery $(\mathrm{n})$ & 8 & 18 \\
Parkinson $(\mathrm{n})$ & 1 & 1 \\
\hline
\end{tabular}

There was no association between the gynaecological history and sensation markers during conventional-UDS, or with the presence of DO, but there was a significant association between gynaecological surgery and the feeling of urgency (Pearson $\chi^{2}, p=0.028$ )

The mean HADS-anxiety subscore is 6.77 ( $\mathrm{Cl} \mathrm{0-18,} \mathrm{s.d.} \mathrm{4.1)} \mathrm{the} \mathrm{mean} \mathrm{HADS-}$ depression score 4.66 (Cl 0-19, s.d. 4.1) and the mean total HADS is 11.43 (s.d. 7.8) (Table 2). No significant association was found considering HADS anxiety and depression scores with variables of the voiding diaries or medical history

\section{HADS and conventional-UDS}

There was a significant association of total HADS, using a cut-off of 15, with DO (Pearson $\left.\chi^{2}, p=0.019\right)$. A separate analysis for men and women was performed; there was no association in men ( $p=0.101)$ but a significant association in women $(p=0.035)$. HADS anxiety using a cut-off of 8 was significantly associated with the presence of DO (Pear- 
son $\chi^{2}, p=0.018$.) Regarding men, there was no association found ( $\left.p=0.494\right)$, nevertheless anxiety was significantly associated with DO in women $(p=0.012)$. No association was found between HADS depression and DO, however there was a significant association between HADS depression, using cut-off 8 , and the feeling of urgency $\left(\chi^{2}, p=0.028\right)$.

Table 2. Psychometric results in patients with detrusor overactivity (DO+) and no detrusor overactivity (DO-) in urodynamic investigation. HADS-A= Hospital Anxiety and Depression Scale-Anxiety subscore, HADS-D= Hospital Anxiety and Depression Scale-Depression subscore. ${ }^{*}=$ statistically significant Pearson $\chi^{2}$.

\begin{tabular}{llll}
\hline HADS & All $n=74$ & $\mathrm{DO}+, \mathrm{n}=32$ & DO-, $\mathrm{n}=42$ \\
\hline HADS-A & 6.77 (s.d. 4.09) & $\begin{array}{l}7.91 \text { (s.d. 3.99) } \\
\mathrm{P}=0.018^{*}\end{array}$ & 5.9 (s.d. 3.99) \\
HADS-D & 4.66 (s.d. 4.14) & $\begin{array}{l}5.66 \text { (s.d. 3.77) } \\
\mathrm{P}=0.135\end{array}$ & 3.90 (s.d. 4.29) \\
Total HADS & 11.43 (s.d. 7.78) & $\begin{array}{l}13.56 \text { (s.d. 7.35) } \\
\mathrm{P}=0.019 *\end{array}$ & 9.81 (s.d. 7.78) \\
& & \\
\hline
\end{tabular}

\section{Urinary frequency with or without DO}

There was a significant association between the mean voided volume and the presence of DO (t-test, $p=0.047$ ) (Table 3). Self-reported incontinence and DO was not statistically significant (Pearson $\left.\chi^{2} p=0.574\right)$, neither was self-reported incontinence associated with UDS proven incontinence (Urgency Pearson $\chi^{2}, p=0.306$, SI Pearson $\chi^{2}, p=0.259$ ). Patients with DO had a smaller cystometric bladder capacity compared to patients without DO (287 vs. $381 \mathrm{ml}$, t-test, $\mathrm{p}=0.004)$. In addition, a significant difference in volume of strong desire $(257 \mathrm{ml}$ vs. $330 \mathrm{ml}, \mathrm{p}=0.034)$ was shown between both groups. The volume of first desire (ttest, $p=0.31)$, normal desire $(p=0.805)$ or post-void residual urine $(p=0.499)$ were not statistically different. Patients with DO were significantly older (59.2 vs. 52.6, p=0.047).

Table 3. Characteristics of patients with detrusor overactivity versus patients without detrusor overactivity on conventional urodynamic investigation. DO = detrusor overactivity, UDS= urodynamic study, ${ }^{*}=$ statistically significant

\begin{tabular}{llll}
\hline & $\begin{array}{l}\text { Urinary Frequency } \\
\text { with DO }\end{array}$ & $\begin{array}{l}\text { Urinary Frequency } \\
\text { without DO }\end{array}$ & P-value \\
\hline Number of patients & 32 & 42 & $0.047^{*}$ \\
Age (years) & 59 & 53 & $0.047^{*}$ \\
Mean voided volume on bladder diaries (ml) & 166 & 202 & 0.57 \\
Frequency on voiding diary & 11.6 & 11.1 & 0.57 \\
Incontinence on voiding diary (n) & 24 & 29 & 0.77 \\
History of urgency (n) & 23 & 20 & $0.004^{*}$ \\
Bladder capacity on UDS (ml) & 286 & 381 & 0.387 \\
Volume first desire on UDS (ml) & 175 & 200 & $0.034^{*}$ \\
Volume strong desire (ml) & 257 & 330 & \\
\hline
\end{tabular}




\section{DISCUSSION}

This pilot study revealed a significant association between the total HADS scores and DO, between HADS anxiety scores and DO and between HADS depression scores and the feeling of urgency. To our knowledge, the present study is the first to report an association between affective symptoms and DO.

Available literature shows higher depression and anxiety rates in urodynamically proven SUI, DO or sensory urgency compared to the normal controls. However, no difference were reported with respect to affective symptoms among these diagnoses ${ }^{14}$. Other studies did not reveal any difference considering affective symptoms and urodynamic diagnoses ${ }^{15,16}$.

Given that evidence comparing affective symptoms with urodynamic parameter is limited, our investigation may inform the field further on the association of urodynamics and affective complaints. Until now, the associations between idiopathic urgency incontinence proven by urodynamics and depression, but not anxiety, are described, but not the association between DO and affective disorders ${ }^{17,18}$. Patients with DO have lower quality of life scores and a lower mental health domain score on the SF 36 compared to normal urodynamics ${ }^{19}$

In the present study, urinary frequency was not associated with DO on conventionalUDS. This is consistent with results from the literature; frequency alone has been proven to be a poor predictor of DO ${ }^{20}$. Nevertheless, it has been hypothesised that frequency originates from cognitive strategies to avoid intense urgency and incontinence ${ }^{21}$. Hashim and colleagues described a reasonable correlation between OAB symptoms and urodynamic diagnosis. DO was present in $69 \%$ of the men and $44 \%$ of women with OAB dry, this was $90 \%$ and $44 \%$ in $O A B$ wet ${ }^{20}$. Associations between urgency symptoms scores with the presence of DO have been described, although we did not use validated symptoms scores ${ }^{22}$.

An explanation for the association between affective disorders and DO could be that they share common anatomical or biological pathways. Functional MRI during conventional-UDS in young women with UUI and DO showed increased brain activity in the Anterior Cingulate Gyrus (ACG) ${ }^{23}$. The ACG is a part of the Anterior Cingulated Cortex (ACC) were elevated activity is found in young people with depression ${ }^{24}$. Another common pathway can be the hypothalamic-pituitary-adrenal axis. Dysregulation of this axis, with increased corticotroponin-releasing factor (CRF) increased adrenocorticotropic hormone (ACTH) and increased cortisol plays a role in depression and anxiety ${ }^{25}$. CRF lowered the micturition threshold in a rodent model of DO and anxiety, and administrating a CRF antagonist reduced DO ${ }^{26}$. Additionally, a common denominator could be the serotonergic system. Serotonin depletion might contribute to depression and selective serotonin reuptake inhibitors (SSRIs) are highly effective in treating depression ${ }^{25}$. This complex system also plays various roles in regulation of anxiety ${ }^{27}$. Lowering serotonin levels in the central nervous system leads to urinary frequency and DO in experi- 
mental animal studies ${ }^{28}$. While activation of the central serotonergic system depresses reflex bladder contractions and increase the bladder volume threshold for inducing micturition ${ }^{29}$. Interestingly, a rat model of depression caused by lowering serotonin in the central nervous system, showed symptoms of OAB. This was manifested as urinary frequency due to non-neurogenic detrusor overactivity and lowered bladder capacity compared to controls. This was only seen in female rats. Administrating a SSRI reversed these urinary symptoms ${ }^{30}$. In humans the antidepressant Duloxetine, a serotoninnoradrenaline reuptake inhibitor which increases central serotonin levels, significantly improved frequency and urgency in female OAB patients ${ }^{31}$.

The presented pilot study clearly has its limitations, for example in terms of heterogeneity of the study group, as $O A B$ is a rather broad complex of symptoms. A second limitation is the number of included patients, which makes subgroup analyses difficult to interpret. However, realizing that these data arise from a large tertiary functional urology referral centre, selection bias is possible. Obviously it is retrospective, and this study and its results should stimulate the design of a prospective follow-up study.

In conclusion, this pilot study of comparing psychometric parameters with urodynamic results revealed an association between the total HADS scores and DO, between HADS anxiety scores and DO and between HADS depression scores and the feeling of urgency.

However there is no association between complaints or voiding diaries with anxiety or depression. A conclusion regarding causal relationship cannot be made on the basis of our results. Nevertheless, we hypothesise that common neurobiological pathways with regard to the bladder-brain axis may be the explanation for the association of HADS scores with the urodynamic results. Further research is needed to elucidate which parts of the bladder-brain axis are involved in affective disorders as well as bladder function and preferably longitudinal in order to be able to make statements about causal relationships. 


\section{REFERENCES}

1. DSM-V and Diagnostic and statistical manual of mental disorders, American Psychiatric Association 5th ed. 2013, Washington, DC.

2. Ustun, T.B., et al., Global burden of depressive disorders in the year 2000. Br J Psychiatry, 2004. 184: p. 386-92.

3. Wang, P.S., et al., Twelve-month use of mental health services in the United States: results from the National Comorbidity Survey Replication. Arch Gen Psychiatry, 2005. 62(6): p. 629-40.

4. $\quad$ Engel, W.J., Uropsychiatry. J Mich State Med Soc, 1964. 63: p. 273-7.

5. Macaulay, A.J., et al., Micturition and the mind: psychological factors in the aetiology and treatment of urinary symptoms in women. Br Med J (Clin Res Ed), 1987. 294(6571): p. 540-3.

6. Abrams, P., et al., The standardisation of terminology of lower urinary tract function: report from the Standardisation Sub-committee of the International Continence Society. Neurourol Urodyn, 2002. 21(2): p. 167-78.

7. Elinoff, V., et al., Symptom-specific efficacy of tolterodine extended release in patients with overactive bladder: the IMPACT trial. Int J Clin Pract, 2006. 60(6): p. 745-51.

8. Coyne, K.S., et al., The burden of lower urinary tract symptoms: evaluating the effect of LUTS on healthrelated quality of life, anxiety and depression: EpiLUTS. BJU Int, 2009. 103 Suppl 3: p. 4-11.

9. Haylen, B.T., et al., An International Urogynecological Association (IUGA)/International Continence Society (ICS) joint report on the terminology for female pelvic floor dysfunction. Neurourol Urodyn, 2010. 29(1): p. 4-20.

10. Guralnick, M.L., et al., Objective differences between overactive bladder patients with and without urodynamically proven detrusor overactivity. Int Urogynecol J, 2010. 21(3): p. 325-9.

11. Vrijens, D., et al., Affective symptoms and the overactive bladder - A systematic review. J Psychosom Res, 2015;78:95-108.

12. Zigmond, A.S. and R.P. Snaith, The hospital anxiety and depression scale. Acta Psychiatr Scand, 1983. 67(6): p. 361-70.

13. Schafer, W., et al., Good urodynamic practices: uroflowmetry, filling cystometry, and pressure-flow studies. Neurourol Urodyn, 2002. 21(3): p. 261-74.

14. Macaulay, A.J., R.S. Stern, and S.L. Stanton, Psychological aspects of 211 female patients attending a urodynamic unit. J Psychosom Res, 1991. 35(1): p. 1-10.

15. Chiara, G., et al., Psychological investigation in female patients suffering from urinary incontinence. Int Urogynecol J Pelvic Floor Dysfunct, 1998. 9(2): p. 73-7.

16. Lagro-Janssen, A.L., F.M. Debruyne, and C. Van Weel, Psychological aspects of female urinary incontinence in general practice. Br J Urol, 1992. 70(5): p. 499-502.

17. Zorn, B.H., et al., Urinary incontinence and depression. J Urol, 1999. 162(1): p. 82-4.

18. Stach-Lempinen, B., et al., Severe depression determines quality of life in urinary incontinent women. Neurourol Urodyn, 2003. 22(6): p. 563-8.

19. Duggan, P., Urodynamic diagnoses and quality of life in women presenting for evaluation of urinary incontinence. Aust N Z J Obstet Gynaecol, 2011. 51(5): p. 416-20.

20. Hashim, H. and P. Abrams, Is the bladder a reliable witness for predicting detrusor overactivity? J Urol, 2006. 175(1): p. 191-4; discussion 194-5.

21. Harvey, J., et al., The relationship between cognition and sensation in determining when and where to void: the concept of cognitive voiding. BJU Int, 2012. 110(11): p. 1756-61.

22. Chung, S.D., et al., Urgency severity scale could predict urodynamic detrusor overactivity in patients with overactive bladder syndrome. Neurourol Urodyn, 2011. 30(7): p. 1300-4.

23. Griffiths, D., et al., Cerebral control of the bladder in normal and urge-incontinent women. Neuroimage, 2007. 37(1): p. 1-7.

24. Kerestes, R., et al., Functional brain imaging studies of youth depression: A systematic review. Neuroimage Clin, 2013. 4: p. 209-231. 


\section{Chapter 5}

25. Nemeroff, C.B., The neurobiology of depression. Sci Am, 1998. 278(6): p. 42-9.

26. Klausner, A.P., et al., The role of corticotropin releasing factor and its antagonist, astressin, on micturition in the rat. Autonomic Neuroscience: Basic and Clinical, 2005. 123(1-2): p. 26-35.

27. Gordon, J.A. and R. Hen, The serotonergic system and anxiety. Neuromolecular Med, 2004. 5(1): p. 2740.

28. Steers, W.D. and K.S. Lee, Depression and incontinence. World journal of urology, 2001. 19(5): p. 351357.

29. de Groat, W.C., Influence of central serotonergic mechanisms on lower urinary tract function. Urology, 2002. 59(5 Suppl 1): p. 30-6.

30. Lee, K.S., et al., Alterations in voiding frequency and cystometry in the clomipramine induced model of endogenous depression and reversal with fluoxetine. Journal of Urology, 2003. 170(5): p. 2067-2071.

31. Steers, W.D., et al., Duloxetine compared with placebo for treating women with symptoms of overactive bladder. BJU Int, 2007. 100(2): p. 337-45. 




\section{Chapter}

\section{Screening for depression and anxiety in}

patients with storage or voiding dysfunction:

A retrospective cohort study predicting outcome of sacral neuromodulation

Drossaerts JM, Vrijens DMJ, Leue C, Schilders I, Van Kerrebroeck Ph, van Koeveringe GA. Neurourol Urodyn. 2016 Nov;35(8):1011-1016 


\section{ABSTRACT}

\section{Aims}

It has been reported that somatic treatment in patients with affective symptoms has a higher risk of failure. The aim was to investigate whether affective symptoms could predict the outcome of sacral neuromodulation (SNM) for lower urinary tract symptoms (LUTS).

\section{Methods}

All patients that underwent a SNM evaluation between 2006 and 2013 and filled out a Hospital Anxiety and Depression Score (HADS) before treatment, were included. Chisquare analysis and bivariate logistic regression were used to assess associations and predictive value.

\section{Results}

Eighty-six patients were included, 65 females and 21 males. Most patients, 66, had overactive bladder syndrome (OAB). The remaining 20 patients suffered from nonobstructive urinary retention (NOR). 39 OAB patients and 17 NOR patients, had a normal total HADS score before treatment. Significantly more patients showed abnormal HADS-D ( $p=0.047)$ and HADS-A ( $p=0.015)$ scores in the OAB group compared to the NOR group. Success of SNM could not be predicted by the HADS score $p=0.464$ (after 1 year $p=0.446$ ). Subsequent analysis revealed that abnormal HADS score was not related to the occurrence of SNM adverse events.

\section{Conclusions}

The present study did not reveal a significant relation between abnormal HADS score and failure of the SNM test period in a mixed group of OAB and NOR patients. However, differences between $\mathrm{OAB}$ and NOR patients concerning affective symptoms were present. It is known that psychological factors play a role in the severity of LUTS, but they may not predict SNM outcome. 


\section{INTRODUCTION}

Lower urinary tract symptoms (LUTS) due to functional urological causes often coincide with some type of psychological or psychiatric manifestation. This may even be the case when patients without a documented psychiatric history are considered. Furthermore, also in healthy subjects emotional influences can impact voiding and often will lead to an increase in urgency and frequency. In general the association of a somatic disease with a depressive disorder is not uncommon and affects $25 \%$ of hospital inpatient populations ${ }^{1}$. The co-occurrence and association between common psychiatric conditions (i.e. affective symptoms) and overactive bladder is extensively described in literature ${ }^{2,3}$. Depression and anxiety disorders are affective psychiatric disorders and diagnosed according to the DSM-IV criteria.

LUTS encompasses the overactive bladder syndrome (OAB) described by the International Continence Society (ICS) as urgency, frequency and/or nocturia with or without urgency incontinence ${ }^{4}$. Non-obstructive urinary retention (NOR), also LUTS, can be caused by detrusor underactivity, detrusor-bladder neck dyssynergia, detrusor-external sphincter dyssynergia, dysfunctional voiding and non-relaxing urethral sphincter obstruction. Concerning NOR there is a minimal choice of treatment options, like pelvic floor physiotherapy or alpha blockers, and patients are often obliged to self-catheterise. Behavioural therapy and/or pharmacotherapy are the mainstay of treatment for OAB. About $50 \%$ of $O A B$ patients gain benefit from pharmacotherapy. A next stage in treatment for $O A B$ patients can be intravesical injection of botulinum toxin or sacral neuromodulation $(\mathrm{SNM})^{5}$. SNM is recommended as a secondary treatment option for patients with $O A B$ or NOR, if conservative treatments either fail or lead to insurmountable adverse events ${ }^{6}$. A SNM test period evaluates improvement during several weeks ${ }^{7}$. Success rates of SNM vary between $60-80 \%$. In our department on average $70 \%$ of the patients benefit from SNM ${ }^{8}$. Although several predictive factors for success in SNM have been identified, the test period has been found to be the only constant factor in predicting success $^{9,10}$. Nonetheless, a more reliable selection of patients with highest chance of treatment success (or highest chance of treatment failure) could be improved by identifying other factors, or a combination of factors, that can predict outcome. Predicting outcome can guide optimal treatment choice and the exclusion of patients with high chance of treatment failure may contribute to cost effectiveness ${ }^{11,12}$. The determination of such predictive factors has been subject of several studies and affective comorbidity may be one of these predictive factors ${ }^{13-16}$.

\section{AIM OF STUDY}

Up until this study, the screening for depressive and anxiety symptoms and SNM outcome has not been of main interest in former studies. In this study, the relation be- 
tween the presence of affective symptoms and treatment outcome is assessed. It is hypothesised that there is a difference in outcome of SNM between patients with normal and with aberrant anxiety or depressive symptoms scores. Thus, the presence of affective symptoms could be a predictive factor in SNM outcome on the short term, or even on long term.

\section{METHODS}

\section{Setting}

The present study is based on a retrospective evaluation of patients with LUTS presented at the Urology department of the Maastricht University Medical Centre (MUMC). All patients were diagnosed and treated according to standard clinical practice. Patients with refractory OAB or NOR were offered SNM. All patients that underwent an SNM test period between 2006 and 2013 and filled out a Hospital Anxiety and Depression Scale (HADS) questionnaire before treatment, were included. The HADS questionnaire is a validated questionnaire in hospital patients, and was part of the standardised intake procedure at the Pelvic Care Centre (PCC) Maastricht ${ }^{17}$.

\section{Affective symptom screening}

The HADS screens for the presence of depressive and anxiety symptoms in somatically ill patients ${ }^{18}$. It contains 14 items 4-point Likert type scales, which are divided into two subscales that assess depression and anxiety. A normal HADS score is defined as 0-7 per sub-score. A score of 8-10 per sub-score indicates mild mood disturbance, a score of 11 14 suggests moderate symptoms and a sub-score of $15-21$ adverts to a severe mood disturbance ${ }^{18}$. The HADS has high concurrent validity with the Beck Depression Inventory (BDI) and the Clinical Anxiety Scale (CAS) questionnaires. All patients with a psychiatric history were not undergoing active treatment at the time of filling out the HADS and (the work-up for) SNM treatment.

\section{Treatment evaluation}

SNM is performed in two stages, first the tined lead is placed and after approximately 4 weeks the tined lead is removed or a neuromodulator is implanted. No temporary lead placement (percutaneous nerve evaluation; PNE) procedures are performed in our centre because of their inferiority ${ }^{7}$. SNM treatment success is defined as a reduction of one or more micturition symptoms of $\geq 50 \%$, compared to baseline, determined by comparing voiding diaries ${ }^{7}$. Following treatment success, permanent implantation is performed. Patient characteristics, voiding diaries, urodynamic measurements and 
adverse events were documented. Urodynamic studies were performed using Medical Measurements Systems (MMS B.V., Enschede, The Netherlands) equipment.

\section{Statistical analysis}

Analyses were conducted using SPSS software 20, IBM Corporation. Chi-square analysis and odds ratio (OR) assessed association between categorical variables. The HADS-D and HADS-A scales were either dichotomized with a cut-off point of 8 (normal vs. abnormal) or a cut-off point of 11 (normal or mild vs. moderate or severe) or categorized as a four level ordinal variable: normal 0-7; mild 8-10, moderate 11-14 or severe 15-21. The dichotomisation with a cut-off point of 8 was elected. Additionally the total HADS score was used to calculate associations ${ }^{19}$. Binary logistic regression was applied to assess predictive value of having a successful SNM test period by HADS scores (continuous scale). Logistic regression analyses were subsequently performed to adjust for and estimate the effect of the covariates age (continuous) and gender and psychiatric history (categorical). Sensitivity analyses were performed with psychiatric status and use of antidepressant or anxiety repressing medication to avoid misclassification of persons with medically treated affective disorders in remission.

\section{RESULTS}

Treatment evaluation and affective symptom screening

Eighty-six patients, 65 females and 21 males with a mean age of 58 years (s.d.12), were included who filled out a HADS questionnaire prior to SNM treatment. Most patients, 66 (77\%), had OAB and the remaining 20 (23\%) patients suffered from NOR. In 52 patients (60\%) SNM was successful during test period. Of these 47 (90\%) were still treated successfully 1 year after the implantation.

Of all patients included 29 (34\%) had a documented psychiatric history prior to HADS (and thus prior to SNM). Psychiatric history consisted out of one or more of following disorders: depression, anxiety disorder, pain syndrome and post-traumatic stress disorder. Amongst OAB patients 39 (59\%) had a normal total HADS score, of the NOR patients 17 (85\%) had a normal total HADS score. Significantly more patients had abnormal HADS-D $(p=0.047)$ and abnormal HADS-A $(p=0.015)$ in the OAB group compared to the NOR group (Figure 1). 
Chapter 6

Panel A. HADS-A subscore

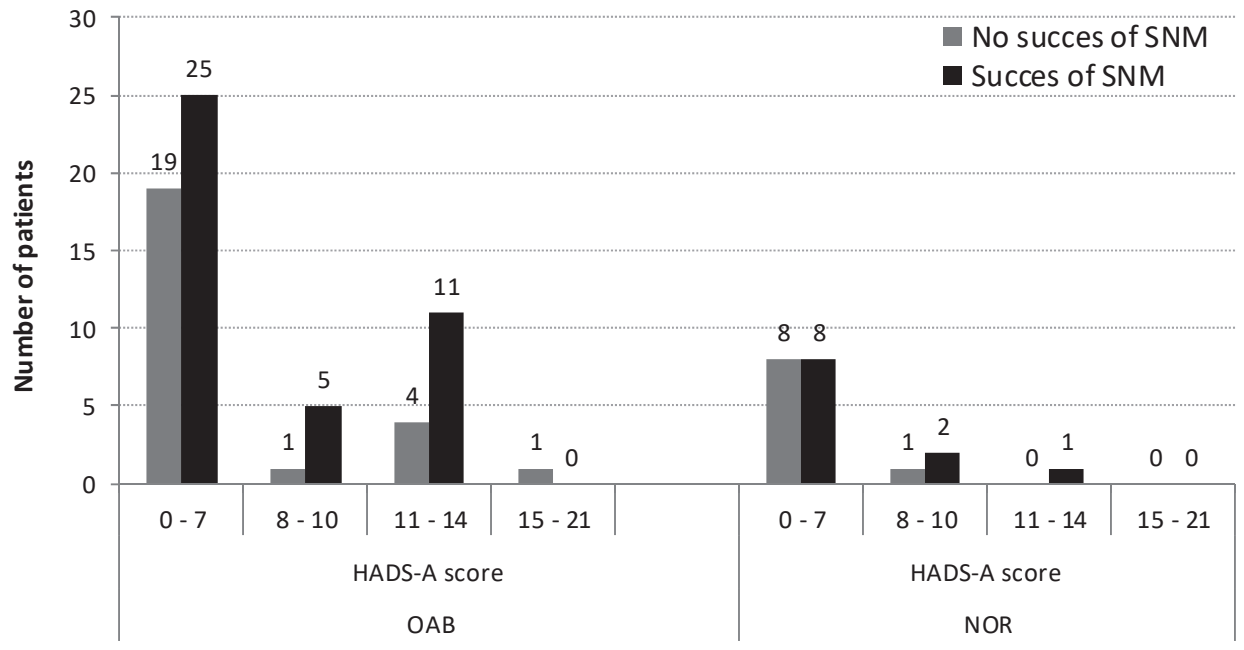

Panel B. HADS-D subscore

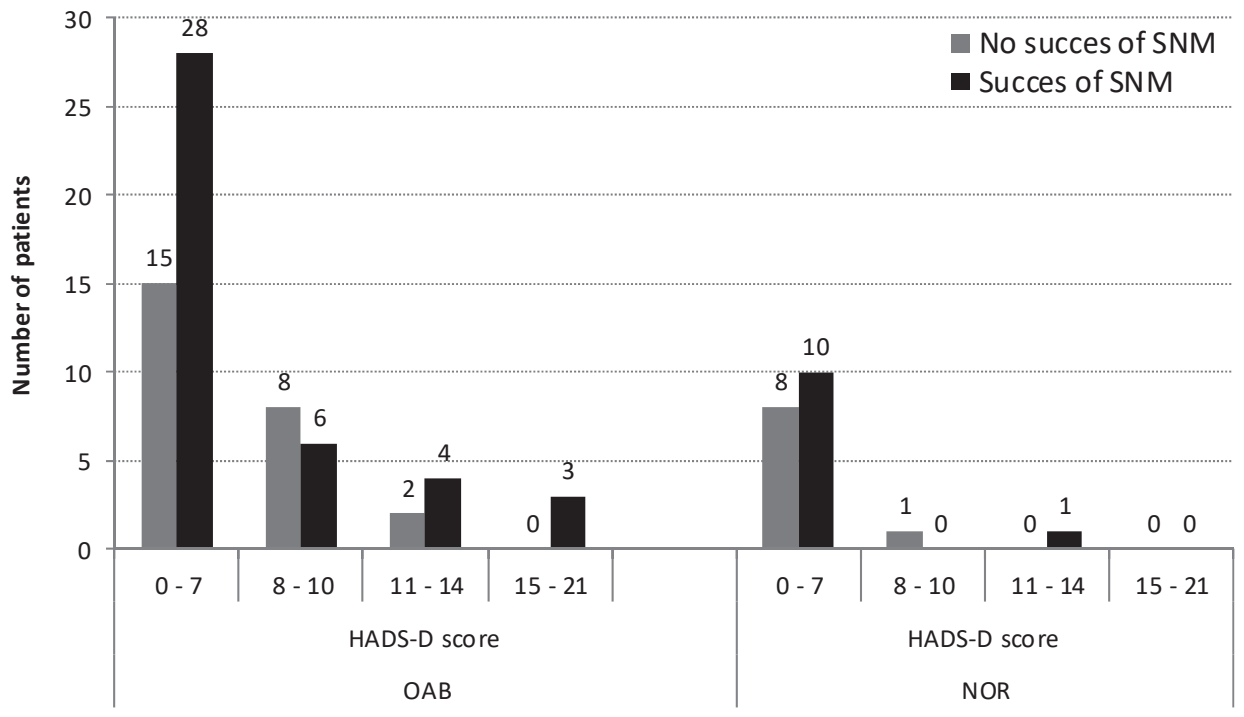


Panel C. Total HADS score

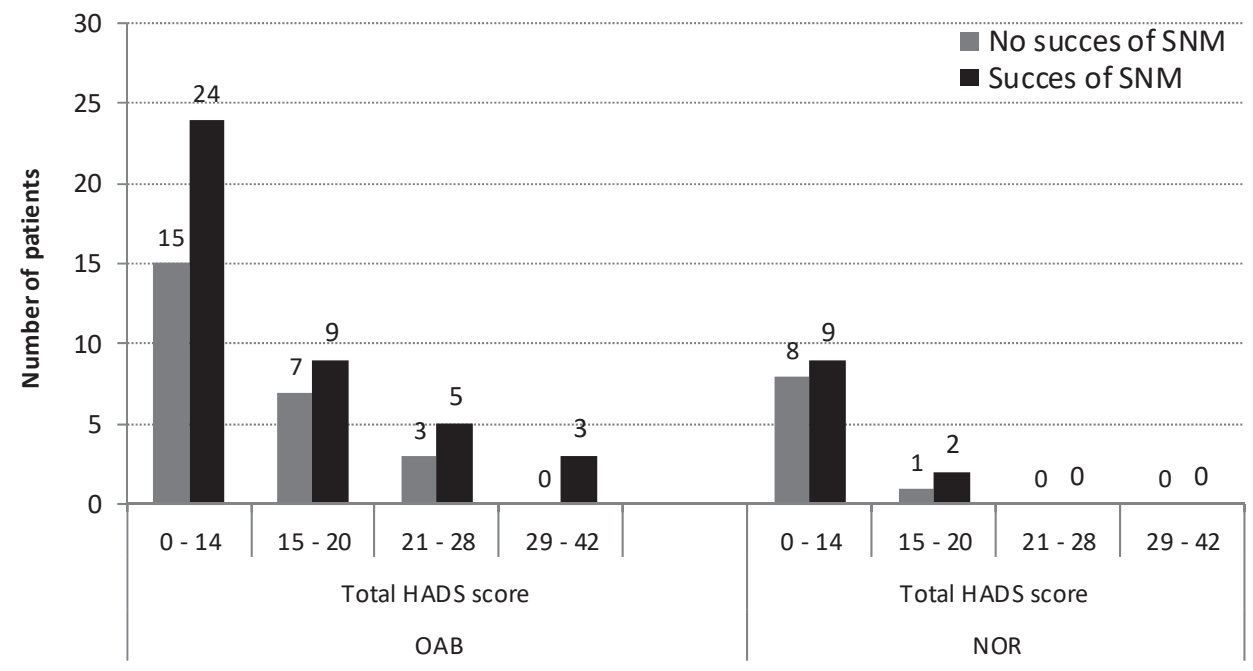

Figure1. SNM success / unsuccessful by HADS score. HADS scores are split for specific scores: panel A. showing the depression (HADS-D) subscores and panel B. showing the anxiety (HADS-A) subscores. Panel C. depicts total HADS score amongst patients. SNM success is depicted separate for OAB and NOR groups. The cut off value for an abnormal HADS-A or HADS-D score is 8, the cut off for the total HADS is 15.

\section{Micturition diaries}

According to the micturition diaries, 52 patients had a bladder capacity of 200-500 ml (61\%) (OAB s.d. $=174$, NOR s.d. = 309). Most patients $(n=60)$ had a voiding frequency of more than 7 times a day (70\%), 10 patients had a voiding frequency of less than 4 and 16 patients had a normal voiding frequency of 4-7 times a day. 52 patients (60\%) had 2 or more incontinence episodes a day. The other 34 patients (40\%) had no incontinence. If self-catheterization was performed (by the 20 NOR patients), the patient drained most often a volume of 200-500 ml.

\section{Urodynamics}

In $52 \mathrm{OAB}$ and 18 NOR patients the urodynamic recordings were present in the patient files. In 21 (32\%) OAB patients' detrusor overactivity (DO) was recorded. According to the urodynamics, 46 (54\%) patients had a maximum bladder capacity of 200-500 ml, 10 of $500-1000 \mathrm{ml}$ and 13 a capacity of 0-200 ml. In 32 (37\%) patients the volume at micturition command was $200-500 \mathrm{ml}$. NOR patients most often had a post void residual volume of $200-500 \mathrm{ml}$ during the urodynamic recording. 


\section{Ordinal variable analyses}

Categorical associations and effect estimates were calculated. There was no significant association between success of SNM, and 1) the somatic urological indication (OAB or NOR), 2) the age of the patients or 3) their prior psychiatric history. Aberrant HADS-D or HADS-A and success of SNM after 1 year (overall and per indication) were not associated. Higher HADS scores and DO on conventional urodynamics were not related. In Table 1 the significant associations between other variables are depicted, these represent the secondary outcomes of this study. There was a significant association between the HADS-D and the HADS-A score. Total HADS and HADS-D score were significantly higher in patients with OAB compared to NOR patients (OR 3.8-4.5). Furthermore, total HADS, HADS-D and HADS-A was significantly higher in patients with prior documented psychiatric history.

In OAB patients with an abnormal total HADS and/or HADS-D and/or HADS-A urgency urinary incontinence occurred significantly more often than in the OAB patients with normal HADS scores.

\section{Logistic regression analyses}

Logistic regression did not reveal significant prediction of SNM success by different variables. Interaction of total HADS, HADS-A and HADS-D with psychiatric history and with each other was examined in several models, but revealed no interaction of relevance. The interaction terms did not add to the prediction model. Success of SNM could not be predicted by HADS score, $p=0.464$. Moreover, the persistent treatment effect after 1 year could not be predicted by HADS score, $p=0.446$.

\section{Adverse events}

Ten patients reported an adverse event during the follow-up period. These patients complained of pain at the neuromodulator implantation site. The pain was managed without surgical intervention in all of these patients. Five patients lost some of the initial improvement. No other adverse events were reported. Subsequent logistic regression analysis revealed that (ab)normal HADS score was not related to the occurrence of adverse events of SNM ( $p$-value 0.2 to 0.6 , depending on the specific logistic regression model used).

\section{DISCUSSION}

The present retrospective cohort study is the first to investigate the potential predictive value of affective complaints in relation to treatment failure of SNM. The analysis revealed that the presence of affective symptoms in a group of patients treated with SNM, did not lead to a higher chance of treatment failure. 
Table I. Associations between preoperatively HADS score and postoperatively success of SNM (using solely categorical variables). Several secondary associations were also calculated, relating the HADS to indication, urgency incontinence and psychiatric history.

\begin{tabular}{|c|c|c|c|c|}
\hline Association between & Chi-square $\left(\chi^{2}\right)$ & $\begin{array}{l}\text { Spearman's } \\
\text { correlation }\left(r_{s}\right)\end{array}$ & Odds Ratio (95\% Cl) & $\mathrm{p}$-value \\
\hline \multicolumn{5}{|l|}{ Success of SNM treatment } \\
\hline $\begin{array}{l}\text { HADS I success SNM } \\
\text { (total HADS, HADS-D, HADS-A) }\end{array}$ & \multicolumn{3}{|l|}{ not associated } & $0.204-0.690$ \\
\hline $\begin{array}{l}\text { HADS | success SNM } \\
\text { after } 1 \text { year } \\
\text { (total HADS, HADS-D, HADS-A) }\end{array}$ & \multicolumn{3}{|l|}{ not associated } & $0.136-0.869$ \\
\hline \multicolumn{5}{|l|}{ Indication } \\
\hline $\begin{array}{l}\text { Abnormal total HADS | OAB } \\
\text { predominance }\end{array}$ & 4.54 & -0.230 & $3.8(1.6-10.5)$ & 0.033 \\
\hline $\begin{array}{l}\text { Abnormal HADS-D | OAB } \\
\text { predominance }\end{array}$ & 4.30 & -0.231 & $4.5(1.6-15.7)$ & 0.032 \\
\hline $\begin{array}{l}\text { Abnormal HADS-A | OAB } \\
\text { predominance }\end{array}$ & \multirow{2}{*}{\multicolumn{2}{|c|}{ not associated }} & & 0.482 \\
\hline Incontinence & & & & \\
\hline $\begin{array}{l}\text { Abnormal total HADS | } \\
\text { occurrence of incontinence in } \\
\text { OAB patients }\end{array}$ & 10.50 & 0.399 & $16.3(1.9-132.6)$ & 0.001 \\
\hline \multicolumn{2}{|c|}{$\begin{array}{l}\text { Abnormal HADS-D | occurrence } 7.61 \\
\text { of incontinence in OAB patients }\end{array}$} & 0.340 & $11.8(1.4-96.2)$ & 0.006 \\
\hline \multicolumn{2}{|c|}{$\begin{array}{l}\text { Abnormal HADS-A I occurrence } 6.97 \\
\text { of incontinence in OAB patients }\end{array}$} & 0.325 & $10.9(1.3-88.8)$ & 0.008 \\
\hline \multicolumn{5}{|l|}{ Psychiatric history } \\
\hline $\begin{array}{l}\text { Abnormal total HADS I } \\
\text { presence of psychiatric history }\end{array}$ & 10.85 & 0.355 & $4.8(1.8-12.6)$ & 0.001 \\
\hline \multicolumn{2}{|c|}{$\begin{array}{l}\text { Abnormal HADS-D | presence of } 8.40 \\
\text { psychiatric history }\end{array}$} & 0.312 & $3.9(1.5-10.4)$ & 0.004 \\
\hline \multicolumn{2}{|c|}{$\begin{array}{l}\text { Abnormal HADS-A | presence of } 7.83 \\
\text { psychiatric history }\end{array}$} & 0.302 & $4.0(1.5-10.6)$ & 0.005 \\
\hline $\begin{array}{l}\text { Abnormal HADS-D and } \\
\text { abnormal HADS-A }\end{array}$ & 26.98 & 0.560 & $14.8(4.8-45.7)$ & 0.001 \\
\hline
\end{tabular}

Significance level $(\alpha)=5 \%$ : $p<0.05$. Spearman's correlation effect size: $<0.15$ very weak, 0.15-0.25 weak, 0.250.40 moderate, 0.40-0.75 strong, 0.75-1.0 very strong. Effect estimates are presented as odds ratios (OR) with $95 \% \mathrm{Cl}$. All expected counts in the contingency tables $\geq 5$. Degrees of freedom in all calculations were 1 . Abbreviations: $\mathrm{HADS}=$ hospital anxiety and depression scale, $\mathrm{DO}=$ detrusor overactivity, $\mathrm{OAB}=$ overactive bladder.

The affective symptoms screening tool, the HADS, is not able to predict outcome of SNM treatment. Higher HADS scores neither predict failure in the initial test period nor the late (1 year postoperative) loss of treatment effect. However, there are several meaningful associations between HADS score and LUTS. An affirmation of external validity of the HADS questionnaire is seen since there is a significant association between 
HADS and the presence of psychiatric history in our study population. This indicates that the HADS may be used validly in patients with functional pelvic complaints. Patients with $O A B$ have a 3.8 (odds ratio) times higher chance of having an abnormal HADS score, compared to the NOR patients $(p=0.033)$, which indicates that OAB may be more influenced by pathways of the brain bladder axis than NOR. Furthermore, OAB-wet patients have significantly more often an abnormal HADS-score, compared to OAB-dry patients, which is in accordance with the literature since other studies reported that quality of life is also more affected in patients with OAB-wet, compared to patients with OAB-dry ${ }^{20}$.

In addition, the presence of DO is moderately associated with the occurrence of adverse events in the $O A B$ group. Thus, prediction of patients that will have a very high chance of adverse events should also consider this group of patients ${ }^{21}$. In the past, it has been attempted to identify patients which are more likely to benefit from SNM treatment ${ }^{14}$. Apparently, it is difficult to define reliable predictive factors ${ }^{9,10}$. Complex patients who are suffering from an affective disorder in comorbidity with somatic diseases are reported to have a less optimal course of treatment compared to patients with somatic diseases alone ${ }^{1}$. Although it is known that psychological factors play a role in symptom severity of LUTS as well, this study indicates that at least affective complaints may not predict SNM treatment outcome.

The result of our investigation should be seen in the light of several limitations. First of all it is important to mention that there is a selection bias since most of our patients were referred via the PCC while some others were not. The PCC delivers integrated care which may have led to referral bias of patients considered as more likely to be SNM candidates. Part of which could be reflected by the fact that almost $70 \%$ of patients had a normal HADS-A and HADS-D score. Thus, the real value of preselection cannot be addressed by the outcomes of this research, as no randomised treatment set-up was used.

However, Marcelissen et al. found no evidence that psychological screening for personality traits and psychological status could predict success chances of SNM ${ }^{22}$. A trend was found in patients with a history of psychiatric diseases concerning more often experienced adverse events during permanent SNM treatment ${ }^{22}$. In another study, the level of optimism did not correlate with satisfaction of SNM treatment, change in OAB symptoms, or OAB-related quality of life. On the other hand health-related quality of life, OAB symptoms, depression and body image improved after SNM treatment, suggesting that SNM might also positively contribute to mental health status ${ }^{23}$. Everaert et al. found in $70 \%$ of patients with dysfunctional micturition psychological disturbances. If there was a difference between the test period and the post-implant period in symptom alleviation, this was not dependent upon the occurrence of complications or incomplete response, but was significantly related to a psychiatric disease in history ${ }^{24}$. Furthermore, Yazdany et al. found that patients with worse quality of life at baseline were more likely to have the device explanted, even though these patients showed improvement 
during test period ${ }^{25}$. Everaert et al. reported that patients with a psychiatric history had

significantly more device related pain compared to patients without such history ${ }^{24}$. Moreover, compared to non-depressed patients the odds are three times higher that depressed patients will be non-compliant to an advised medical treatment. This poses a possible risk factor for poor treatment outcome since patients might not adhere to given medical directives ${ }^{26}$.

So far, the understanding that LUTS, especially OAB and affective symptoms coexist frequently can have important implications for the treating clinician ${ }^{2}$. It is recommended that urologists use a validated questionnaire, such as the HADS, to screen for anxiety or depressive symptoms in all patients (eligible for treatment). Having insight into patients' emotional state may improve communication and prevent patient dissatisfaction. The response to medical management for LUTS can be improved by recognising and treating possible underlying affective conditions. Since part of triage has taken place multidisciplinary (including psychiatric expertise) at the PCC, this integrated approach may have influenced outcome in the present study positively. Furthermore, our study design could have possibly failed to identify a group of patients who are less likely to experience symptom improvement and are prone to demand abundant health care utilisation.

\section{CONCLUSION}

In the present retrospective study no significant relation between an elevated HADS score and success or failure of SNM was found in a mixed group of OAB and NOR patients. However, differences between OAB and NOR patients in the occurrence of affective symptoms are present. In future research pre-stratified analysis of OAB and NOR patients should be performed. Our study results and the literature suggest that psychological factors play a role in LUTS severity, but may not predict treatment outcome. Thus, given the current evidence on SNM in complex patients, SNM should not be withheld to patients with affective conditions, but should be offered from an integrated care perspective.

\section{ACKNOWLEDGEMENTS}

The personnel of the Urology department and of the Pelvic Care Centre of the Maastricht University Medical Centre are gratefully acknowledged. 


\section{REFERENCES}

1. Kroenke, K., Patients presenting with somatic complaints: epidemiology, psychiatric comorbidity and management. Int J Methods Psychiatr Res, 2003. 12(1): p. 34-43.

2. Vrijens, D., et al., Affective symptoms and the overactive bladder-a systematic review. J Psychosom Res, 2015. 78(2): p. 95-108.

3. Dolat, M.T. and A.P. Klausner, UROPSYCHIATRY: The Relationship Between Overactive Bladder and Psychiatric Disorders. Current Bladder Dysfunction Reports, 2013. 8(1): p. 69-76.

4. Abrams, P., et al., The standardisation of terminology of lower urinary tract function: report from the Standardisation Sub-committee of the International Continence Society. Neurourol Urodyn, 2002. 21(2): p. 167-78.

5. Smits, M.A., et al., Sacral neuromodulation in patients with idiopathic overactive bladder after initial botulinum toxin therapy. J Urol, 2013. 190(6): p. 2148-52.

6. Lucas, M.G., et al., EAU guidelines on surgical treatment of urinary incontinence. Actas Urol Esp, 2013. 37(8): p. 459-72.

7. Marcelissen, T., et al., Is the screening method of sacral neuromodulation a prognostic factor for longterm success? J Urol, 2011. 185(2): p. 583-7.

8. van Kerrebroeck, P.E., et al., Results of sacral neuromodulation therapy for urinary voiding dysfunction: outcomes of a prospective, worldwide clinical study. J Urol, 2007. 178(5): p. 2029-34.

9. Koldewijn, E.L., et al., Predictors of success with neuromodulation in lower urinary tract dysfunction: results of trial stimulation in 100 patients. J Urol, 1994. 152(6 Pt 1): p. 2071-5.

10. Scheepens, W.A., et al., Predictive factors for sacral neuromodulation in chronic lower urinary tract dysfunction. Urology, 2002. 60(4): p. 598-602.

11. Leong, R.K., et al., Cost-effectiveness analysis of sacral neuromodulation and botulinum toxin A treatment for patients with idiopathic overactive bladder. BJU Int, 2011. 108(4): p. 558-64.

12. Mayr, C.A. and J.P. Shepherd, Cost-effectiveness of novel therapies for overactive bladder. Expert Rev Pharmacoecon Outcomes Res, 2014. 14(4): p. 527-35.

13. Anger, J.T., et al., Predictors of implantable pulse generator placement after sacral neuromodulation: who does better? Neuromodulation, 2014. 17(4): p. 381-4; discussion 384.

14. Drossaerts, J., et al., The value of urodynamic tools to guide patient selection in sacral neuromodulation. World J Urol, 2015. 33(11): p. 1889-95.

15. Peeters, K., et al., Long-term follow-up of sacral neuromodulation for lower urinary tract dysfunction. BJU Int, 2014. 113(5): p. 789-94.

16. Peters, K.M., et al., Does patient age impact outcomes of neuromodulation? Neurourology and Urodynamics, 2013. 32(1): p. 30-36.

17. Berghmans, B., et al., Prevalence and triage of first contact pelvic floor dysfunction complaints in male patients referred to a Pelvic Care Centre. Neurourol Urodyn, 2016 Apr;35(4): p.487-91.

18. Zigmond, A.S. and R.P. Snaith, The hospital anxiety and depression scale. Acta Psychiatr Scand, 1983. 67(6): p. 361-70.

19. Norton, S., et al., The Hospital Anxiety and Depression Scale: a meta confirmatory factor analysis. J Psychosom Res, 2013. 74(1): p. 74-81.

20. Hashim, H. and P. Abrams, Overactive bladder: an update. Curr Opin Urol, 2007. 17(4): p. 231-6.

21. Kessler, T.M., et al., Urologists' referral attitude for sacral neuromodulation for treating refractory idiopathic overactive bladder syndrome: discrete choice experiment. Neurourol Urodyn, 2014. 33(8): p. 12406 .

22. Marcelissen, T.A., et al., Psychological and psychiatric factors as predictors for success in sacral neuromodulation treatment. BJU Int, 2011. 108(11): p. 1834-8.

23. Levin, P.J., et al., Psychosocial factors related to the use of InterStim(R) for the treatment of refractory overactive bladder. Female Pelvic Med Reconstr Surg, 2014. 20(5): p. 272-5. 
24. Everaert, K., et al., Patient satisfaction and complications following sacral nerve stimulation for urinary retention, urge incontinence and perineal pain: a multicenter evaluation. Int Urogynecol J Pelvic Floor Dysfunct, 2000. 11(4): p. 231-5; discussion 236.

25. Yazdany, T., N. Bhatia, and J. Nguyen, Determining outcomes, adverse events, and predictors of success after sacral neuromodulation for lower urinary disorders in women. Int Urogynecol J, 2011. 22(12): p. 1549-54.

26. DiMatteo, M.R., H.S. Lepper, and T.W. Croghan, Depression is a risk factor for noncompliance with medical treatment: meta-analysis of the effects of anxiety and depression on patient adherence. Arch Intern Med, 2000. 160(14): p. 2101-7. 

Chapter

\section{Patient reported outcome after treatment of urinary incontinence in a multidisciplinary pelvic care clinic}

Vrijens DMJ, Spakman JI, van Koeveringe GA, Berghmans B.

Int J Urol 2015;22:1051-7 


\section{ABSTRACT}

\section{Objectives}

To assess patient reported outcome and satisfaction regarding urinary incontinence (UI) one year after the end of treatment in a multidisciplinary pelvic care clinic.

\section{Methods}

A total of 647 patients with urinary incontinence seen in an academic multidisciplinary pelvic care clinic were prospectively included in a specific triage system. Patientreported improvement of urinary incontinence and patient satisfaction were assessed by telephone survey one year after end of treatment.

\section{Results}

Just $15.6 \%$ of patients presented with one single pelvic floor problem, most had two or three pelvic floor dysfunctions simultaneously, such as urinary incontinence, pelvic organ prolapse and constipation or faecal incontinence. One year after the end of treatment, of 440 responders (68\%), 18.2\% reported no remaining complaints, and $33.4 \%$ reported only one complaint. Patients reported a significant improvement of the mean severity (scale $0-10)$ of UI from $7.2( \pm 1.6)$ pre-treatment $(=T 0)$ to $4.3( \pm 3.0)$ one year after end of treatment $(p<0.001)$. A total of $20.6 \%$ of patients reported no UI after one year $(p<0.001)$ and $27.6 \%(p<0.001)$ stopped using incontinence pads. Patients claimed high satisfaction with the clinic and care received, with $35.8 \%$ being 'satisfied' and $45.5 \%$ 'very satisfied'.

\section{Conclusion}

At one year after the end of treatment, one out of five patients with urinary incontinence recovered completely and more than one out of four stopped using incontinence absorption pads. One out of three patients went from having a multifactorial health problem to a mono-factorial health problem. High satisfaction rates were reported by more than four out of five patients. 


\section{INTRODUCTION}

According to the definition of the International Continence Society (ICS), the prevalence of urinary incontinence (UI) in women is $25-29 \%$, making it one of the most prevalent pelvic floor dysfunction ${ }^{1}$. Historically, this problem is treated in a monodisciplinary way by gynaecologists or urologists without combining their knowledge and experience ${ }^{2}$. However, patients often suffer from multiple pelvic floor dysfunctions (PFDs). A large cross-sectional study found, for instance, an increased risk for faecal incontinence (FI) in women with UI (odds ratio 4.9). Pelvic organ prolapse (POP) was also accompanied by an increased risk for $\mathrm{UI}$ and $\mathrm{FI}$ (respectively Odds Ratios 3.8 and 4.2 ) $^{3}$. Even more complicated is the fact that treating only one problem in patients with multiple problems can aggravate or unmask other complaints. For example, correcting rectal prolapse can thus induce or unmask cystocele and/or $\mathrm{UI}^{4}$. Therefore, monodisciplinary treatment might be suboptimal, as relevant literature and current clinical practice increasingly show the importance of holistic assessment and evaluation of PFDs, and advocate multidisciplinary treatment ${ }^{5-7}$. However, objective data to support the multidisciplinary approach are scarce ${ }^{8-11}$. Therefore we carried out the present study in our multidisciplinary pelvic care clinic (Pelvic Care Centre, PCC).

We analysed the Patient Reported Outcome (PRO) of interventions in the PCC one year after conservative or invasive treatment for UI. PRO is a measurement of a patient's health status that comes directly from the patient and provides evidence of treatment benefit from the patients perspective ${ }^{12}$. PRO has gained increasing attention both in clinical research and in treatment evaluation by health care insurance and regulatory agencies. In the UK for example, routine collection of patient-reported outcome measures (PROMs) is used to measure the performance of health-care providers ${ }^{13}$. Furthermore, evaluation of patient related outcome can result in change of treatment ${ }^{14}$. For example, in prostate cancer, PRO have become the cornerstone of measurements of the side effects of treatment ${ }^{15}$.

The objectives of the present study included the assessment of PRO for different treatments of $\mathrm{UI}$ and other functional pelvic complaints. Additionally, we assessed patient satisfaction regarding their current health status regarding $\mathrm{UI}$ and treatment received in a multidisciplinary academic centre.

\section{METHODS}

Our PCC is a multidisciplinary academic pelvic care clinic that serves as a large secondary and tertiary referral centre. Additionally, it serves as the (only) local hospital that general practitioners refer patients to. The main goals of the PCC are to comprehensively direct patients to the right (para)medical specialist(s) and to address all significant problems simultaneously or in adequate order. In order to achieve this, patients were 
subjected to a triage system after referral ${ }^{16}$. After triage, the triage data were analysed using a specially developed digital model. Complaints were then categorised in six categories: UI, lower urinary tract symptoms (LUTS), pelvic organ prolapse (POP), $\mathrm{FI}$, other problems with defecating (e.g. constipation), sexual problems and/or menstrual problems. Based on the significant complaints, the relevant medical specialists were selected. Patients could be seen by multiple specialists in succession or simultaneously. Complex problems were discussed in weekly multidisciplinary meetings (Figure 1). The triage system of our centre is described in more detail in another article ${ }^{16}$.

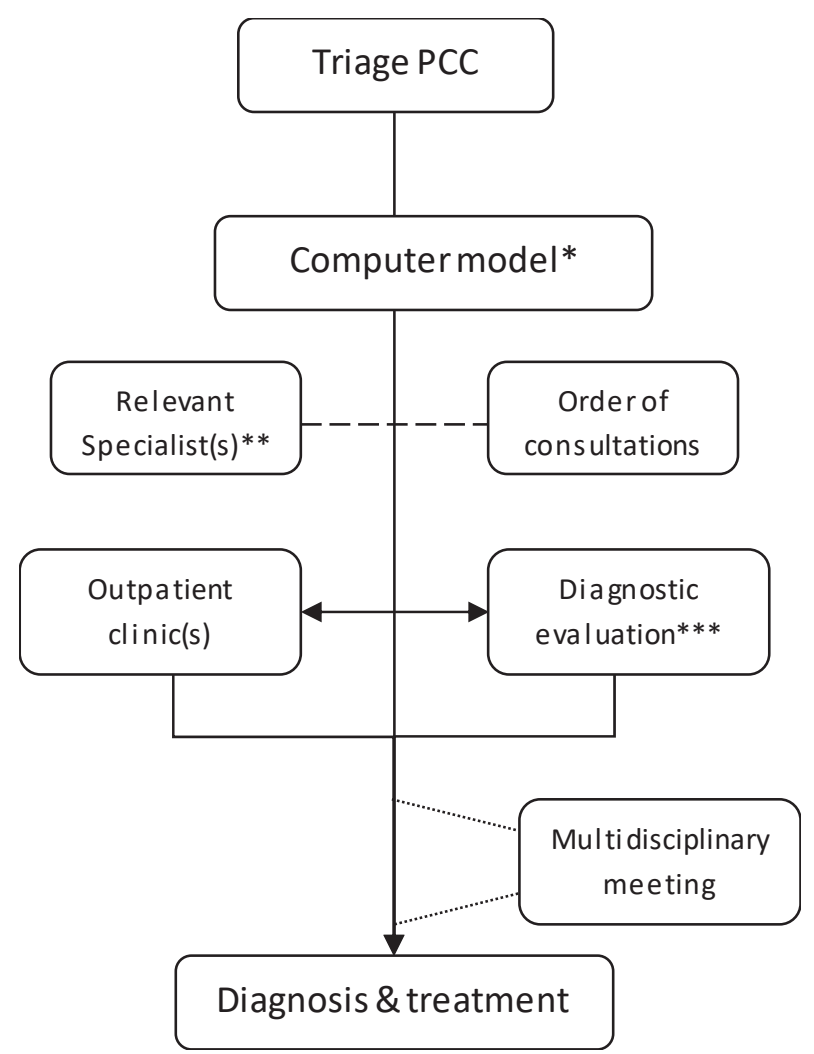

Figure 1. Pelvic Care model

*computer model categorizes complaints in 6 categories: urinary incontinence (UI), lower urinary tract symptoms (LUTS), prolapse (POP), problems with defecating (e.g. constipation), faecal incontinence (FI), sexual problems and/or menstrual problems.

** Relevant specialists; urologist, gynaecologist, colorectal surgeon, sexologist, psychiatrist, physiotherapist, incontinence nurse.

*** Diagnostic evaluation and all consultations in outpatient clinics are on the same day, if possible. 
Data were prospectively collected using a standardised triage system. The analysis and evaluation of the treatment results were carried retrospectively. The local medical ethics committee reviewed the study and approved it (protocol number METC 13-4-082).

Patients seen for UI in the PCC in the period 2006-2012, were identified and selected for inclusion using the hospital's administrative codes for UI. TO is the date of triage and T1 is discharge from the PCC. T2 is the survey date, approximately one year after the end of treatment. The treatments received were categorised into two categories: (i) conservative and (ii) invasive treatment. Conservative treatment consisted of only one or more of following treatments: expectative, behavioural therapy, pharmacotherapy; that is, antimuscarinics, pelvic physiotherapy and pessary treatment. All surgical interventions; for example, trans-obturator tape, tension-free vaginal tape, bulking agents, botulinum toxin injections, sphincter prostheses or neuromodulation by sacral nerve stimulation, were considered invasive. Patients who were deceased at $\mathrm{T} 2$ were excluded from the present study.

A structured telephone survey was carried out by three specialized continence nurses and a research assistant. They were blinded for medical information and previous treatment and received specific protocol instructions not to inquire about the previous situation. The survey was developed and tested and previously used for reports to insurance companies. Intra-and inter-tester reliability was confirmed. The amount of contact attempts per patient was maximised at three. The interview started after informed consent and took 5-10 minutes (Appendix 1).

Details of medical history and patient characteristics were derived from the triage questionnaire and medical records. Severity of UI was assessed at TO during triage and later at T2 during the interview by asking patients to score their current UI on a scale from 0 to 10. Patients were considered to have stress urinary incontinence (SUI) when losing urine during coughing, laughing, sneezing and/or exercising, derived from triage data. Urgency urinary incontinence (UUI) was defined by losing urine during or right after feeling urgency; that is, irresistible urge. Mixed urinary incontinence (MUI) was considered a combination of both SUI and UUI in subjectively similar measures. Patients with predominant SUI or UUI were characterised as having SUI or UUI, respectively. Patients with UI that could not be defined by either of these conditions were assigned to the category 'other'.

\section{Data analysis}

Data were analysed using SPSS statistics for Windows, version 21.0 (IBM Corp. Released 2012. IBM SPSS Statistics for Windows, Version 21.0. Armonk, NY: IBM Corp). The data were coded using a numeric sequence, to keep the researchers blinded during the analysis.

Patient characteristics at TO were analysed with unpaired T-tests and Pearson's chisquared test $\left(\chi^{2}\right)$. Changes in severity of $\mathrm{UI}$ and in pad use between TO and T2 were analysed using paired T-tests and Wilcoxon signed rank tests. Changes per type of UI 
and per treatment were assessed with repeated measurements ANOVA and by analysing the differences between TO and T2 with Kruskal-Wallis tests. Differences in severity per risk factor between TO and T2 were also analysed with repeated measurements ANOVA and Mann-Whitney U-tests. $\mathrm{P}<0.05$ was considered to show a statistically significant difference (two-sided).

\section{RESULTS}

During the study period, 2377 patients with UI visited the clinic. From every year a random representative sample of patients who finished their treatment at PCC were asked to participate. Selection was carried out by computerized randomization based on patient number. The characteristics of the 647 patients are shown in Table 1. Respectively, 281 (43.3\%) and 101 (15.6\%) patients were primarily seen by an urologist or a gynaecologist. A total of 191 (29.5\%) patients were seen by more than one specialist during the first consultation. Most patients , 421 (65.1\%), were treated conservatively, 226 (34.9\%) patients were treated surgically. A total of 90 (17.6\%) patients started conservative therapy, but required surgery later on. Of all operated patients, just 13 (2.0\%) received a combined surgical procedure (Table 2). Patients who had received any previous treatment for $\mathrm{UI}$ were more often treated surgically instead of conservatively $(p=0.008)$. Furthermore, they required more surgeries per patient $(p<0.001)$. The survey response rate at T2 was 440 (68.0\%), with no differences in patient characteristics, compared with non-responders. The main reasons for inclusion failure were not answering the phone after three attempts and incorrect telephone numbers.

Table 1. Patient characteristics

\begin{tabular}{|c|c|c|c|c|}
\hline Characteristics & Men $(n=64)$ & Women $(n=583)$ & $\begin{array}{l}\text { Total } \\
(n=647)\end{array}$ & $\begin{array}{l}\mathrm{P} \text { value (difference } \\
\text { between men and } \\
\text { women) }\end{array}$ \\
\hline Mean age in years (range) & $58.7(23.3-82.8)$ & $57.9(17.6-92.7)$ & $58.0(17.6-92.7)$ & 0.630 (T-test) \\
\hline \multicolumn{5}{|l|}{ Referral by } \\
\hline GP & 39 & 375 & $414(64.0 \%)$ & \multirow[t]{2}{*}{$0.592(\times 2)$} \\
\hline Specialist & 25 & 208 & $233(36.0 \%)$ & \\
\hline First consult for UI & 43 & 424 & $467(72.2 \%)$ & \multirow[t]{2}{*}{$0.489(x 2)$} \\
\hline Unknown & 4 & 23 & $27(4.2 \%)$ & \\
\hline \multicolumn{5}{|l|}{ Type UI * } \\
\hline Stress & $12(18.8 \%)$ & $219(37.6 \%)$ & $231(35.7 \%)$ & \multirow{5}{*}{$0.000(x 2)$} \\
\hline Urge & $22(34.4 \%)$ & $102(17.5 \%)$ & $124(19.1 \%)$ & \\
\hline Mixed & $4(6.3 \%)$ & $177(30.4 \%)$ & $181(28.0 \%)$ & \\
\hline Other & $14(21.9 \%)$ & $21(3.6 \%)$ & $35(5.4 \%)$ & \\
\hline Unknown & $12(18.8 \%)$ & $64(11.0 \%)$ & $76(11.7 \%)$ & \\
\hline
\end{tabular}




\begin{tabular}{|c|c|c|c|c|}
\hline Characteristics & Men $(n=64)$ & Women $(n=583)$ & $\begin{array}{l}\text { Total } \\
(n=647)\end{array}$ & $\begin{array}{l}\text { P value (difference } \\
\text { between men and } \\
\text { women) }\end{array}$ \\
\hline \multicolumn{5}{|l|}{ Severity subjective (0-10) } \\
\hline Mean (s.d.) & $7.2(1.6)$ & $7.2(1.6)$ & $7.3(1.6)$ & \multirow[t]{2}{*}{0.693 (T-test) } \\
\hline Unknown & $8(12.5 \%)$ & $61(10.5 \%)$ & $69(10.7 \%)$ & \\
\hline \multicolumn{5}{|c|}{ Self-reported UI episodes per $24 \mathrm{~h}$} \\
\hline$\leq 1$ episode $24 \mathrm{~h}$ & $16(25.0 \%)$ & $114(19.6 \%)$ & $130(20.1 \%)$ & \multirow[t]{4}{*}{$0.148(\times 2)$} \\
\hline 2-3 episodes $24 \mathrm{~h}$ & $10(15.6 \%)$ & $157(26.9 \%)$ & $167(25.8 \%)$ & \\
\hline$\geq 4$ episodes $24 \mathrm{~h}$ & $19(29.7 \%)$ & $214(36.7 \%)$ & $233(36.1 \%)$ & \\
\hline Unknown & $19(29.7 \%)$ & $98(16.8 \%)$ & $117(18.0 \%)$ & \\
\hline Use of incontinence pads & $18(28.1 \%)$ & $439(75.3 \%)$ & $457(70.6 \%)$ & \multirow[t]{2}{*}{$0.000(\times 2)$} \\
\hline Unknown & $17(26.6 \%)$ & 75 (12.9\%) & $92(14.2 \%)$ & \\
\hline \multicolumn{5}{|l|}{ Complaints $* *$} \\
\hline Only UI & $7(10.9 \%)$ & $73(12.5 \%)$ & $80(12.4 \%)$ & \multirow{9}{*}{$\begin{array}{l}\text { Expected counts } \\
\text { too small for } \chi 2 \\
\text { test. }\end{array}$} \\
\hline$U I+P \& M$ & $28(43.8 \%)$ & $346(53.5 \%)$ & $374(57.8)$ & \\
\hline $\mathrm{UI}+\mathrm{FP}$ & $4(6.3 \%)$ & $25(4.3 \%)$ & $29(4.5 \%)$ & \\
\hline$U I+M \& S$ & $3(4.7 \%)$ & $8(1.4 \%)$ & $11(1.7 \%)$ & \\
\hline$U I+P \& M+F P$ & $1(1.6 \%)$ & $63(10.8 \%)$ & $64(9.9 \%)$ & \\
\hline$U I+P \& M+M \& S$ & $10(15.6 \%)$ & $29(5.0 \%)$ & $39(6.0 \%)$ & \\
\hline$U I+F P+M \& S$ & 0 & $9(1.5 \%)$ & $9(1.4 \%)$ & \\
\hline All complaints & $4(6.3 \%)$ & $12(2.1 \%)$ & $16(2.5 \%)$ & \\
\hline unknown & $7(10.9 \%)$ & $18(3.1 \%)$ & $25(3.8 \%)$ & \\
\hline \multicolumn{5}{|l|}{ Childbirths } \\
\hline 1 & - & $77(13.2 \%)$ & - & \multirow[t]{5}{*}{-} \\
\hline 2 & - & $219(37.6 \%)$ & - & \\
\hline$\geq 3$ & - & $132(22.6 \%)$ & - & \\
\hline Episiotomy & - & $241(41.3 \%)$ & - & \\
\hline Rupture & - & $173(29.7 \%)$ & - & \\
\hline \multicolumn{5}{|l|}{ Medical History } \\
\hline Hysterectomy & - & $127(21.8 \%)$ & - & - \\
\hline Diabetes Mellitus & $3(4.7 \%)$ & $52(8.9 \%)$ & $55(8.5 \%)$ & $0.397(\times 2)$ \\
\hline COPD/Asthma & $7(10.9 \%)$ & $114(19.6 \%)$ & $121(18.7 \%)$ & $0.230(\times 2)$ \\
\hline Burnout/depression/anxiety & $15(23.4 \%)$ & $209(35.7 \%)$ & $224(34.6 \%)$ & $0.167(\chi 2)$ \\
\hline
\end{tabular}

* Stress UI: urine loss when coughing, laughing, and/or exercising. Also stress UI when stress component more prevalent than urgency component. Urgency UI: UI when feeling urgency. Also urgency UI when urgency component more prevalent than stress component. Mixed: Combination of stress and urgency UI, both equally prevalent. Other UI: UI not defined by one of these conditions.

**UI (urinary incontinence), P\&M (prolapse and/or micturition problems), FP (faecal problems; obstipation and/or faecal incontinence), M\&S (Menstruation and/or sexual problems)

Mean patient-reported severity declined from $7.2( \pm 1.6)$ to $4.3( \pm 3.0)$ (p 0.005). A total of $20.6 \%$ of patients with UI reported absence of UI at T2 $(p<0.001)$, and $27.6 \%$ stopped using incontinence absorption materials $(p<0.001)$. A total of $6.6 \%$ used only one pad per 24 hours at T2 $(p<0.001)$. The mean number of pads in 24 hours at T2, was $2.6( \pm 3.1)$. 


\section{Chapter 7}

Table 2. Conservative or invasive treatment

\begin{tabular}{llll}
\hline & $\begin{array}{l}\text { Primary conservative } \\
(\mathrm{n}=511)\end{array}$ & $\begin{array}{l}\text { Primary invasive } \\
(\mathrm{n}=136)\end{array}$ & $\mathrm{P}$ value \\
\hline Mean severity $(0-10)$ & 7.2 & 7.4 & $\mathrm{P}=0.413(\mathrm{~T}$-test) \\
Had previous treatment & $109(21.3 \%)$ & $44(32.5 \%)$ & $\mathrm{P}=0.004(\chi 2)$ \\
Number of surgeries & & & \\
1 & $81(15.8 \%)$ & $97(71.3 \%)$ & $\mathrm{P}=0.000(\chi 2)$ \\
2 & $9(1.8 \%)$ & $32(23.5 \%)$ & \\
$\geq 3$ & 0 & $7(5.1 \%)$ & \\
Ul surgery $*$ & $59(11.5 \%)$ & $79(58.1 \%)$ & $\mathrm{P}=0.000(\chi 2)$ \\
Prolapse surgery $* *$ & $40(7.8 \%)$ & $66(48.5 \%)$ & $\mathrm{P}=0.000(\chi 2)$ \\
Combined surgery & $5(1.0 \%)$ & $8(5.9 \%)$ & Expected counts too \\
Uro + gyn & $1(0.2 \%)$ & $3(2.2 \%)$ & small for $\chi 2$ test \\
Gyn + surg & $4(0.8 \%)$ & $5(3.7 \%)$ & \\
\hline
\end{tabular}

*UI surgery: TOT, TVT, fascia sling, (pro-)ACT, macroplastique, botulinum, laser coagulation, AMS sphincter prosthesis, neuromodulator, percutaneous tibial nerve stimulation (PTNS), Burch colposuspension

** Prolapse surgery: cystocele correction, rectocele correction (including MESH repairs), sacrospinal fixation, hysterectomy, enterocele correction, rectopexy.

Uro +gyn = combined surgery by urologist and gynaecologist. Gyn + surg = combined surgery by gynaecologist and surgeon).

Severity per UI type reported by patients was significantly different before and after treatment $(p<0.001)$. Compared to UUI and MUI, the biggest improvement was seen in patients with SUI, (from mean $7.3( \pm 1.5)$ to 3.7 ( \pm 3.1$)$ ) (Figure 2). There was no significant difference in improvement between men and women $(p=0.90)$.

Patients without previous treatments for UI reported a slightly lower mean (s.d.) severity at the start $(7.2 \pm 1.4)$ compared with patients after previous treatments $(7.5 \pm 1.5$, $p=0.013)$. Also, patients without previous treatments showed more improvement after one year (mean (s.d.) $4.0( \pm 2.9)$ compared to $5.2( \pm 3.2, p=0.013)$. Age did not significantly influence the severity of UI before treatment $(p=0.245)$. The age groups of $30-45$ and 45-60 years improved similarly, whereas patients younger than 30 years or older than 60 years, showed less improvement $(p=0.038)$. In patients younger than 60 years, SUI was the most prevalent type of UI. In patients older than 60 years, UUI and MUI were more prevalent $(p<0.001)$. Conservative or invasive treatment showed no significant differences in mean severity at T2 ( $p=0.130$, Figure 3$)$. 


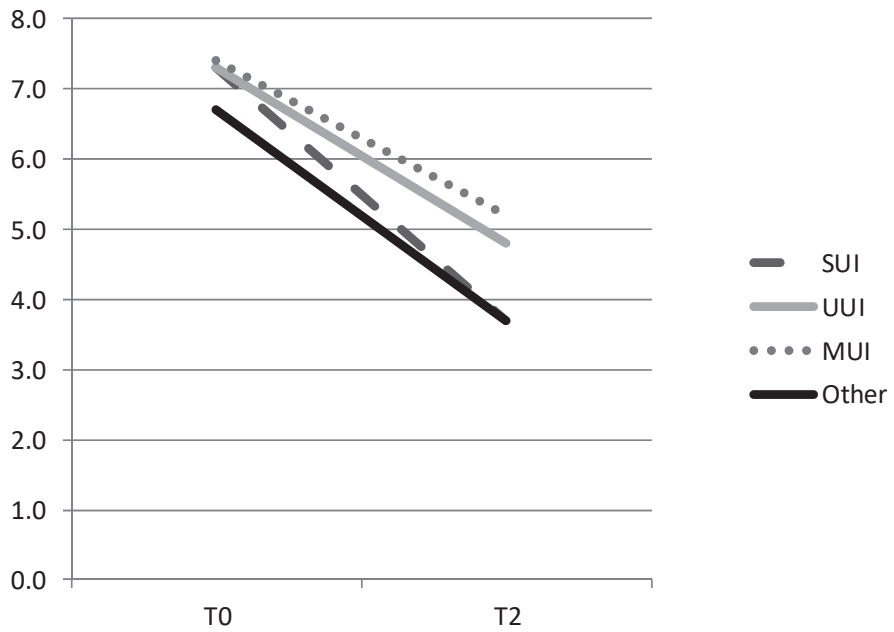

Figure 2. Patient reported severity by type UI

Mean patient reported severity of UI before (TO) and 1 year after end of treatment (T2), by each type of UI incontinence. TO Values: Stress UI 7.3; Urgency UI 7.3; Mixed UI 7.4; Other 6.7. T2 Values: Stress UI 3.7; Urgency UI 4.8; Mixed UI 5.2; Other 3.7 ( $p=0.001)$.

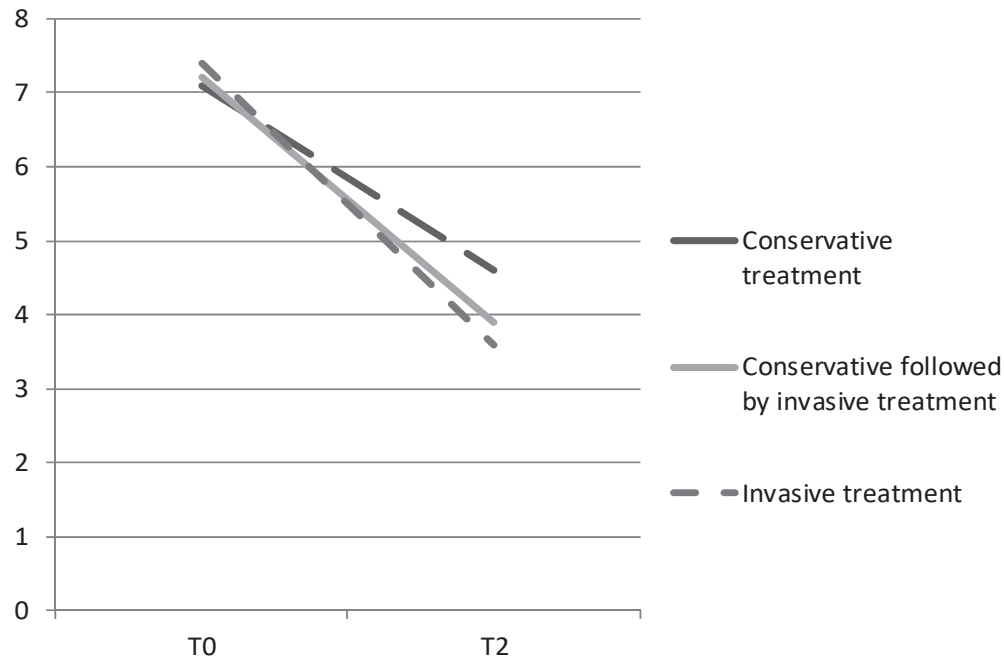

Figure 3. Patient reported severity by previous treatment

Mean patient reported severity of UI before (TO) and 1 year after end of treatment (T2), by patients who had received treatment before being seen in the PCC. T0 values: conservative 7.1 ; invasive 7.4 ; conservative followed by invasive 7.2. T2 values: conservative 4.6; invasive 3.9; conservative followed by invasive 3.6 ( $p=0.130)$.

When analysing severity per risk factor, postmenopausal women started with similar scores ( $p=0.68)$, but showed significantly less improvement $(-2.7)$ than premenopausal women ($3.4, p=0.050)$. Women who had a hysterectomy reported significantly higher mean (s.d.) severity before ( $7.6 \pm 1.6, p=0.018)$ and after treatment $(5.3 \pm 3.1)$ compared with women without $(7.5 \pm 1.6$ and $4.0 \pm 2.9$, respectively) $(p=0.017)$. Patients suffering from (self- 
reported or derived from medical records) burn-out, depression or anxiety before treatment, scored higher in mean (s.d.) severity at baseline $(7.7 \pm 1.3)$, compared to patients without psychological history $(7.1 \pm 1.7, p=0.004)$. However, these psychological factors had no influence on the reported state of UI at T2 ( $p=0.77)$. In addition, there was no significant difference in reported state of UI at T2 in patients with diabetes $(p=0.61)$, asthma and/or $\operatorname{COPD}(p=0.45)$, number of childbirths $(p=0.15)$, episiotomy $(p=0.31)$ or rupture during childbirth $(p=0.15)$ compared with patients without these conditions.

\section{Survey}

At the time of the interview, 137 (31.2\%) of the interviewed patients received additional treatments for UI (Table 3). Of these patients, 104 were again receiving treatment from medical specialists, mostly in our institution (93 patients), with 24 only for pessary maintenance. Of the 303 patients not receiving treatment at the moment of the interview, 38 (8.6\%) patients wished to have a consultation for UI again.

Table 3. T2 Telephone Survey

\begin{tabular}{|c|c|}
\hline & T2 Survey $(n=440)$ \\
\hline Currently receiving treatment & $137(31.1 \%)$ \\
\hline Specialist & $104(23.6 \%)$ \\
\hline Specialist \& physical therapist & $8(1.8 \%)$ \\
\hline GP & $11(2.5 \%)$ \\
\hline GP \& physical therapist & $1(0.2 \%)$ \\
\hline Physical therapist & $13(3.0 \%)$ \\
\hline \multicolumn{2}{|l|}{ Desiring help again for UI * } \\
\hline No & $233(52.7 \%)$ \\
\hline Maybe & $33(7.5 \%)$ \\
\hline Yes & $38(8.6 \%)$ \\
\hline \multicolumn{2}{|l|}{ Complaints** } \\
\hline No complaints & $80(18.2 \%)$ \\
\hline $\mathrm{UI}$ & $109(24.8 \%)$ \\
\hline$P \& M$ & $20(4.5 \%)$ \\
\hline FP & $8(1.8 \%)$ \\
\hline$M \& S$ & $10(2.3 \%)$ \\
\hline$U I+P \& M$ & $66(15.0 \%)$ \\
\hline$U I+F P$ & $30(6.8 \%)$ \\
\hline$U I+M \& S$ & $21(4.8 \%)$ \\
\hline$P \& M+F P$ & $5(1.1 \%)$ \\
\hline$P \& M+M \& S$ & $5(1.1 \%)$ \\
\hline$U I+P \& M+F P$ & $33(7.5 \%)$ \\
\hline$U I+P \& M+M \& S$ & $19(4.3 \%)$ \\
\hline$U I+F P+M \& S$ & $11(2.5 \%)$ \\
\hline$P \& M+F P+M \& S$ & $3(0.7 \%)$ \\
\hline All complaints & $20(4.5 \%)$ \\
\hline
\end{tabular}

*Desiring help for urinary incontinence and not receiving treatment currently.

**UI (urinary incontinence), P\&M (prolapse and/or micturition problems), FP (faecal problems; obstipation and/or faecal incontinence), M\&S (Menstruation and/or sexual problems) 
A total of $18.2 \%$ of the patients reported to have no complaints anymore at $T 2$, and $33.4 \%$ reported only one pelvic floor complaint remaining. The mean (s.d.) number of complaints decreased from $2.2( \pm 1.0)$ to $1.6( \pm 1.2)(p<0.001)$.

A total of $52.5 \%$ of patients reported to be satisfied or very satisfied with their current health status regarding UI (Figure 4). A total of $26.3 \%$ were dissatisfied or very dissatisfied. Patient satisfaction about PCC treatment and the PCC itself is high with $45.5 \%$ 'very satisfied' and $35.8 \%$ being 'satisfied' (Figure 5). These numbers were not influenced by age $(p=0.107)$, treatment $(p=0.792)$ and type of $U I(p=0.084)$.

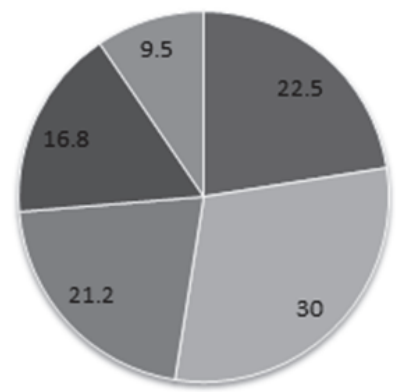
nery sat isfied
= Satisfied
neutral
- Dissat isfied
= Very dissatisfied

Figure 4. T2 Satisfaction health concerning UI

Patient reported measure of satisfaction about their current health situation concerning UI one year after end of treatment (T2).

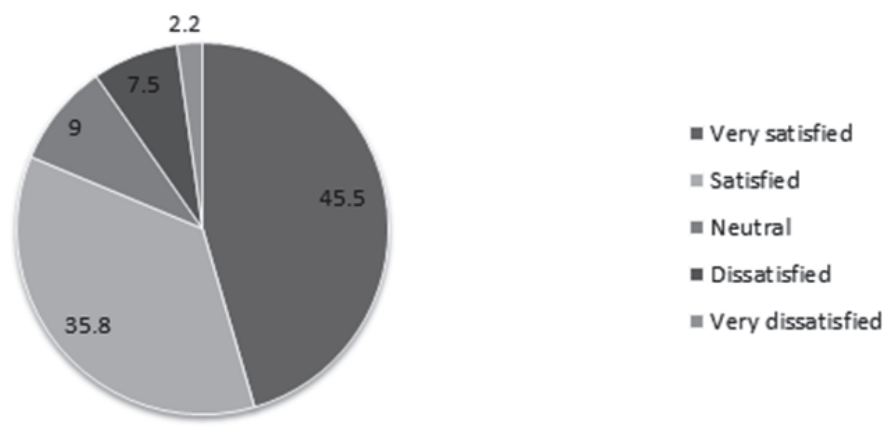

Figure 5. T2 satisfaction treatment and PCC

Patient reported measure of satisfaction about the PCC and the care they received in this centre one year after the end of treatment (T2).

\section{DISCUSSION}

Patients in the PCC were mainly women, often with multiple pelvic floor dysfunctions (PDFs). According to the patient-reported severity, the multidisciplinary treatment methods were effective, although the magnitude of treatment effect depended on the presence of previous treatments, medical history of hysterectomy and type of UI. In this 
difficult to treat population, satisfaction about specific UI health status after treatment was neutral, although satisfaction about their treatment in the PCC and the PCC organisation was (very) high. Our improvement rates were lower than in studies of Padros et al. and Sander et al., who analysed the effect of multidisciplinary conservative treatment of urinary incontinence ${ }^{9,12}$. However, our population had a remarkably high number of patients with previous treatments and multiple PFDs, as our centre serves as a large secondary and tertiary referral centre (Table 1). Other studies carried out in a multidisciplinary pelvic care setting also reported combined problems, but gave no exact numbers ${ }^{10,17}$. Kapoor et al. reported an average of three different pelvic floor complaints per patient, but in this clinic only women with previously diagnosed combined urogynaecological and colorectal problems were treated ${ }^{11}$.

One year after treatment the number of patients with multiple problems had decreased. Furthermore, the number of patients with no complaints or only one remaining complaint, had increased. This indicated that the strategy of the PCC, that is, simultaneous treatment of the individual's pelvic floor problems, had been effective.

\section{Comparisons to mono-disciplinary approaches}

It is difficult to compare multidisciplinary treatments with regular, mono-disciplinary approaches. Most studies focused mainly on SUI and did not report about other PFDs. In a Dutch study, Labrie et al. compared monodisciplinary conservative treatment with surgical intervention (sling) in moderate to severe SUI patients. After physiotherapy, the subjective cure rate was $15.9 \%$ and improvement was $31.7 \%$, after surgery this was $85.2 \%$ and $90.8 \%$, respectively ${ }^{18}$. However, these numbers are not comparable to our patient population, as we also treated patients with other types and severity of UI.

The lower improvement that was reported in the present population might also have been due to the percentage of patients with previous UI treatment that were referred to our tertiary ${ }^{10}$ centre. This is supported by similar results from Al-Singary et al. and Richter et al., both reporting previous UI surgery to be associated with worse long-term outcomes after UI surgery ${ }^{19,20}$.

\section{Patient reported outcome}

The difference in the present study between patient-reported cure $(20.6 \%)$ and patients who no longer required pads (27.6\%) was remarkable. PRO is a complex measure that can be hard to assess objectively or interpret. Subjective improvement of treatment satisfaction often does not correlate with the objective improvement in pad use or outcomes of urodynamic investigations ${ }^{21,22}$. In literature, patient satisfaction seems to depend on many different factors, such as patient's age, patient-physician interaction, influence of the complaints on daily activities and patient's expectations ${ }^{23}$. This could partially explain the different levels of patient's satisfaction about UI, as reported in the present study. 
Studies analysing patient satisfaction for $\mathrm{UI}$ patients in a monodisciplinary setting could not be identified. However, the high satisfaction about the PCC was similar to satisfaction rates in other multidisciplinary pelvic care clinics ${ }^{10,11}$. Although comparisons are hard to make because of different questions asked, our patients satisfaction rates are higher compared with regular care in urology departments in the Netherlands ${ }^{24}$.

This implied that a multidisciplinary approach to PFDs led to high patient satisfaction rates.

\section{Limitations}

Limitations of this study were: risk for selection bias and recall bias. Selection bias was not completely avoidable, because this study only included patients whose treatment ended one year before $\mathrm{T} 2$, patients who still received treatment in our centre were not included in this study. We tried to prevent recall bias by blinding the interviewers and asking only about current pelvic floor complaints. Furthermore, patient data were missing. This could lead to an underestimation of the severity scores and risk factors at TO. Validated questionnaires measuring these kind of multidisciplinary health problems were lacking, therefore outcomes of the present study were based on subjective ratings of incontinence improvement. Another important weakness of our study is the lack of a control group of patients receiving usual care in the same time period.

\section{Implications for the future}

The high prevalence of multiple PFDs in the present study, and decrease after treatment, implies that a multidisciplinary approach of PFDs is likely to be beneficial. Many patients were referred to our tertiary centre after failed previous treatment. Even in this tertiary population, UI improved. The high patient satisfaction about the treatment and the PCC is a further argument in favour of this multidisciplinary approach. More research regarding patient flows, and care lines, clinical pathways and economic analyses, such as cost-effectiveness, is required. One of the objectives remains to reduce repeated clinic visits and to combine diagnostic evaluations on a single day, and to combine surgeries of different specialists in one session if required. Prospective studies, comparing multidisciplinary approach and care-as-usual, should be performed to answer more specific questions on the advantages of a multidisciplinary approach with regard to treatment outcomes.

The present results show that many patients with UI also suffer from other PFDs in overlapping disciplines. This study showed that at 1 year after the end of treatment, one out of five patients with UI reported a complete cure of the incontinence and more than one out of four stopped using incontinence absorption pads. In one out of three patients, their multifactorial health problem was subjectively reduced to a monofactorial health problem. High satisfaction rates were reported by more than four out of five patients. 


\section{REFERENCES}

1. Haylen, B.T., et al., An International Urogynecological Association (IUGA)/International Continence Society (ICS) joint report on the terminology for female pelvic floor dysfunction. Neurourol Urodyn, 2010. 29(1): p. 4-20.

2. Madjar, S., et al., Collaboration and practice patterns among urologists and gynecologists in the treatment of urinary incontinence and pelvic floor prolapse: a survey of the International Continence Society members. Neurourol Urodyn, 2001. 20(1): p. 3-11.

3. Uustal Fornell, E., G. Wingren, and P. Kjolhede, Factors associated with pelvic floor dysfunction with emphasis on urinary and fecal incontinence and genital prolapse: an epidemiological study. Acta Obstet Gynecol Scand, 2004. 83(4): p. 383-9.

4. McNevin, M.S., Overview of pelvic floor disorders. Surg Clin North Am, 2010. 90(1): p. 195-205.

5. Lacima, G. and M. Pera, Combined fecal and urinary incontinence: an update. Curr Opin Obstet Gynecol, 2003. 15(5): p. 405-10.

6. Espejo Maldonado, E., et al., [Stress urinary incontinence. Integral repair of pelvic floor defects]. Arch Esp Urol, 2002. 55(9): p. 1049-55.

7. Chatoor, D., M. Soligo, and A. Emmanuel, Organising a clinical service for patients with pelvic floor disorders. Best Pract Res Clin Gastroenterol, 2009. 23(4): p. 611-20.

8. Kirby, A.C., K.M. Luber, and S.A. Menefee, An update on the current and future demand for care of pelvic floor disorders in the United States. Am J Obstet Gynecol, 2013. 209(6): p. 584 e1-5.

9. Sander, P., et al., Evaluation of a simple, non-surgical concept for management of urinary incontinence (minimal care) in an open-access, interdisciplinary incontinence clinic. Neurourol Urodyn, 2000. 19(1): p. 9-17.

10. Chan, M.C., et al., A retrospective review of patients seen in a multidisciplinary pelvic floor clinic. J Obstet Gynaecol Can, 2010. 32(1): p. 35-40.

11. Kapoor, D.S., et al., Management of complex pelvic floor disorders in a multidisciplinary pelvic floor clinic. Colorectal Dis, 2008. 10(2): p. 118-23.

12. Padros, J., et al., Evaluation of a urinary incontinence unit for community-dwelling older adults in Barcelona: implementation and improvement of the perceived impact on daily life, frequency and severity of urinary incontinence. Z Gerontol Geriatr, 2008. 41(4): p. 291-7.

13. Health, U.S.D.o., et al., Guidance for industry: patient-reported outcome measures: use in medical product development to support labeling claims: draft guidance. Health Qual Life Outcomes, 2006. 4: p. 79.

14. Devlin, N.J., D. Parkin, and J. Browne, Patient-reported outcome measures in the NHS: new methods for analysing and reporting EQ-5D data. Health Econ, 2010. 19(8): p. 886-905.

15. Talcott, J.A., et al., Using patient-reported outcomes to assess and improve prostate cancer brachytherapy. BJU Int, 2014. 114(4): p. 511-6.

16. Berghmans, B., et al., Prevalence and triage of first contact pelvic floor dysfunction complaints in male patients referred to a Pelvic Care Centre. Neurourol Urodyn, 2016 Apr;35 (4): p. 487-91.

17. Resnick, M.J., et al., The Evolution of Self-Reported Urinary and Sexual Dysfunction over the Last Two Decades: Implications for Comparative Effectiveness Research. Eur Urol, 2015. 67(6): p. 1019-25.

18. Labrie, J., et al., Surgery versus physiotherapy for stress urinary incontinence. N Engl J Med, 2013. 369(12): p. 1124-33.

19. Al-Singary, W., M. Arya, and H.R. Patel, Tension-free vaginal tape: avoiding failure. Int J Clin Pract, 2005. 59(5): p. 522-5.

20. Richter, H.E., et al., Patient related factors associated with long-term urinary continence after Burch colposuspension and pubovaginal fascial sling surgeries. J Urol, 2012. 188(2): p. 485-9.

21. Wolters, J.P., A.B. King, and D.E. Rapp, Satisfaction in patients undergoing concurrent pelvic floor surgery for stress urinary incontinence and pelvic organ prolapse. Female Pelvic Med Reconstr Surg, 2014. 20(1): p. 23-6. 
22. Padmanabhan, P. and V.W. Nitti, Female stress urinary incontinence: how do patient and physician perspectives correlate in assessment of outcomes? Curr Opin Urol, 2006. 16(4): p. 212-8.

23. Marschall-Kehrel, D., R.G. Roberts, and L. Brubaker, Patient-reported outcomes in overactive bladder: the influence of perception of condition and expectation for treatment benefit. Urology, 2006. 68(2 Suppl): p. 29-37.

24. Kleefstra, S.M., et al., A core questionnaire for the assessment of patient satisfaction in academic hospitals in The Netherlands: development and first results in a nationwide study. Qual Saf Health Care, 2010. 19(5): p. e24. 


\section{Chapter 7}

\section{APPENDIX}

Pelvic Care Centre Maastricht

Follow-up questionnaire one year after end of treatment (T2)

URINARY INCONTINENCE

T-2: Urinary incontinence

1. Did you suffer from involuntary loss of urine in the last six months?

$0 \square$ No

$1 \square$ Yes

2. Can you rate the bother you experience from the urinary incontinence on a scale from $0-10$, with 0 meaning no bother at all/no complaints, and 10 meaning that the complaint is very severe?

3. In the last 2 weeks did you experience involuntary loss of urine when:

(Multiple answers are possible, therefore ask all the questions. A and B are allowed separate and combined, but answer $C$ excludes answers $A$ and B.)

a. sneezing of coughing, or on effort or physical exertion?

$0 \square$ No

$1 \square$ Yes

$7 \square$ Not relevant

$9 \square$ Unknown

b. feeling urgency, but being unable to reach the toilet in time?

$0 \square$ No

$1 \square$ Yes

$7 \square$ Not relevant

$9 \square$ Unknown

c. feeling urgency and also with effort or physical exertion or on sneezing or coughing?

$0 \square$ No

$1 \square$ Yes

$7 \square$ Not relevant

$9 \square$ Unknown

d. without feeling urgency and without effort or physical exertion or on sneezing or coughing?

$0 \square$ No

$1 \square$ Yes

$7 \square$ Not relevant

$9 \square$ Unknown

4. Do you currently use incontinence pads for the involuntary loss of urine?

$0 \square$ No

$1 \square$ Yes 
5. If yes, in 24 hours, how often do you need to change the incontinence pads because it is wet? (only count wet incontinence pads, exclude changing out of habit or for hygienic reasons)

Number:

Type:

6. Do you currently have any other professional help (e.g. specialist, general practitioner or physical therapist) for the involuntary loss of urine?

- Specialist: $0 \square$ No

$1 \square$ Yes, still/again in Maastricht

$2 \square$ Yes, but in a different hospital, namely:

Doctor

Discipline

Hospital

City

- General practitioner

0

No

$1 \square$ Yes, namely:

Doctor

City

- Physical therapist: 0

No

$1 \square$ Yes, namely:

City

7. Do you currently consider your complaints severe enough to (still) desire professional help from a specialist, general practitioner of physical therapist?

$0 \square$ Not relevant/no complaints

$1 \square$ No, I have urine loss, but do not need help

$2 \square$ Perhaps, I would like to know more/ I am not sure

$3 \square$ Yes, I (definitely) need help

8. Are you satisfied of dissatisfied with your health, considering the involuntary loss of urine?

$0 \square$ Very satisfied

$1 \square$ Satisfied

$2 \square$ Neutral

$3 \square$ Dissatisfied

$4 \square$ Very dissatisfied

$9 \square$ Unknown

9. Are you satisfied or dissatisfied about the treatment provided by the Pelvic Care Centre Maastricht?

$0 \square$ Very satisfied

$1 \square$ Satisfied

$2 \square$ Neutral

$3 \square$ Dissatisfied

$4 \square$ Very dissatisfied

$9 \square$ Unknown/never thought about 


\section{Chapter 7}

10. What are currently your most relevant complaints?

$\begin{array}{lllll} & \text { No } & \text { Yes } & \text { Not relevant } & \text { Unknown } \\ \square \text { Involuntary loss of urine } & 0 & 1 & 9 & 7 \\ \square \text { Micturition difficulties } & \square & \square & \square & \square \\ \square \text { Prolapse complaints } & \square & \square & \square & \square \\ \square \text { Defecation difficulties } & \square & \square & \square & \square \\ \square \text { Involuntary loss of faeces } & \square & \square & \square & \square \\ \square \text { Sexual complaints } & \square & \square & \square & \square \\ \square \text { Menstruation complaints } & \square & \square & \square & \square\end{array}$

Would you like to make any statements concerning the Pelvic Care Centre Maastricht?

\section{Thank you for your time and cooperation!}


Chapter

General discussion 

As a first step, to assess a possible association between lower urinary tract dysfunction (LUTD) and affective symptoms, we conducted a comprehensive qualitative, systematic review on the association between overactive bladder $(O A B)$ and affective symptoms. Based on validated instruments, 43 articles reporting 37 studies were included, overall comprising more than 80,000 individuals. Of these articles, 32 studied the relation between $\mathrm{OAB}$ and depressive symptoms, 2 discussed the relation between $\mathrm{OAB}$ and anxiety, and 9 dealt with both. Regarding the relation between depressive symptoms and $O A B$ a positive association was found in 26 studies. Concerning $O A B$ in relation to anxiety, six studies revealed a positive association. In longitudinal studies, three different directional associations were reported: 1) OAB subjects who developed depressive complaints/anxiety or 2) depressed/anxious subjects who developed OAB, or 3) both.

This analysis, revealed that sufficient good quality evidence exists for a positive association between the co-occurrence of $O A B$ and depressive symptoms, and to a lesser extent also for an association of OAB with anxiety. However, nearly all evaluated studies failed to note longitudinal changes and therefore we are left with a lack of evidence for causality. The association between $\mathrm{OAB}$ and depressive symptoms might be bidirectional in nature. Strong evidence was found concerning a new onset of OAB in depressive patients. On the contrary, it was not possible to make meaningful statements regarding a uni- or bidirectional association between $O A B$ and anxiety, because the evidence was too sparse. Development of urinary incontinence (UI) in subjects with a depression at baseline of the study has been confirmed by two recently published longitudinal studies 1,2 , which were not yet included in our review. The first study is a large population based study in women, which revealed that both depression and anxiety were predictors for the onset of urgency urinary incontinence (UUI). Additionally, UUI was found to be a predictor for the onset of both anxiety and depression, indicating a bidirectional relationship. Furthermore, the association between depression at baseline, and the development of UUI was the strongest for women with higher depression scores ${ }^{1}$. The second study reported that young Australian women with depressive symptoms had a higher prevalence of newly developing UI symptoms over the study period of 10 years ${ }^{2}$. Moreover, the association between depressive symptoms and anxiety and LUTD is confirmed in a recently published, cross sectional study of nearly 72000 nurses ${ }^{3}$ More frequent episodes of UI and greater UI severity were significantly associated with higher prevalence of high scores on depressive symptoms ${ }^{3}$. In another recent cross-sectional study in clinical OAB patients, $27.5 \%$ had depressive symptoms ${ }^{4}$. Hence, the conclusions of our review were confirmed by recent publications. 


\section{PREVALENCE OF AFFECTIVE DISORDERS IN PATIENTS WITH PELVIC FLOOR DYSFUNCTIONS}

In the cross sectional study presented in this thesis, $30 \%$ of patients with pelvic floor dysfunctions (PFDs) at our PCC had an abnormal anxiety score and 20\% had an abnormal depression score. The mean Hospital Anxiety Depression Scale-Anxiety (HADS-A) score was 6.35 and the mean Hospital Anxiety Depression Scale-Depression (HADS-D) score was 4.37, this is higher than in large cross-sectional study in Norway with over 75000 participants. The mean HADS-A score in Norway was 4.0 and the HADS-D score $3.4^{5}$. Our findings are in accordance with the literature concerning psychiatric and somatic comorbidity, for example, in cancer patients, abnormal HADS anxiety scores were found in $30 \%$ of the patients and an abnormal depression scores were seen in $24.1 \%^{6}$. In a cohort of patients with chronic obstructive pulmonary disease (COPD) hospitalized for an acute exacerbation, the prevalence of an abnormal HADS depression score was $44 \%$ at admittance, and $11 \%$ had an abnormal HADS anxiety score ${ }^{7}$. Similar prevalence was detected in patients with oesophageal cancer, 34\% abnormal anxiety score and $23 \%$ abnormal HADS depression score ${ }^{8}$. The prevalence of an abnormal HADS depression score was comparable for patients on haemodialysis with $23.8 \%$ and our cohort of patients with $\mathrm{PFD}^{9}$. The comparison of our results with the literature is displayed in figure 1.

Additionally, in $4.3 \%$ and $2.2 \%$ of the patients at our PCC, the HADS scores of $15-21$ per subscale indicate severe anxiety and depression disturbances respectively. In comparison, $7 \%$ of patients undergoing cardiac surgery had a HADS anxiety score between 11-21 compared to $17.5 \%$ of patients in our cohort ${ }^{10}$.

The prevalence (and association) of depression and anxiety in patients with PFDs is not well studied. In the Unites States (US), a five times higher prevalence of depression (22\%) was reported in women with Pelvic Organ Prolapse (POP) compared to those without ${ }^{11}$. The prevalence of depression among females with advanced pelvic floor dysfunction was much higher in a third world country were a prevalence of $67 \%$ was noted ${ }^{12}$. Furthermore, obese women seeking bariatric surgery with PFDs differed psychosocially from obese women without PFDs. These women with PFDS were more likely to have a history of depression and substance abuse ${ }^{13}$. A qualitive study regarding the emotional burden experienced by women seeking treatment for prolapse revealed that women's psychological well-being is closely related to their pelvic floor symptoms ${ }^{14}$.

In our final regression model, the explained variance of depression and anxiety, measured with the HADS was $12 \%$ and $7.4 \%$ respectively, meaning that, corrected for other contributing variables/factors, $12 \%$ of depression and $7.4 \%$ of anxiety was directly related to PFDs. However, causality was not part of our investigation due to the crosssectional design of our study. Thus, the question whether depression and anxiety are causing PFDs or PDFs are a cause of affective conditions remains unanswered. 


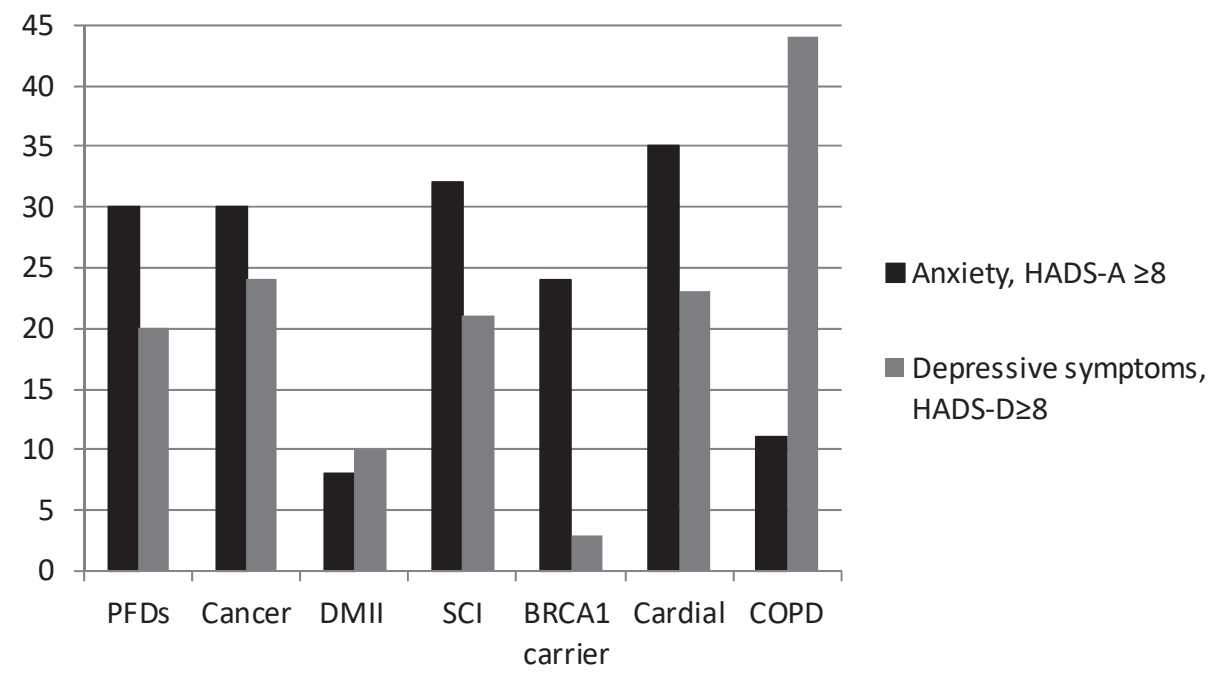

Figure 1. Hospital Anxiety and Depression Scale, per subscale in the literature.

PFDs: pelvic floor dysfunctions, our results. Cancer ${ }^{6}$, DMII: Diabetes Mellitus type $2^{15}$, SCl: Spinal Cord Injury ${ }^{16}$, BRCA1 carrier: Breast Cancer 1 mutation carriers ${ }^{17}$, Cardial ${ }^{18}$, COPD: Chronic Obstructive Pulmonary Disease $^{7}$.

\section{THE ROLE OF PERCEPTION.}

In a normal situation, voiding is usually initiated at a suitable time and place without consciously thinking about it. This is different for patients with OAB. In healthy volunteers normal bladder filling is characterised by a gradual increase in urge, which has been described as first sensation of bladder filling, and normal desire to void up to a strong desire to void ${ }^{19}$, although there is a discussion going on in the literature whether or not this is the normal pattern ${ }^{20}$. Nevertheless, "normal" urge is changed to a pathological type of urge called urgency, in $O A B$ patients, which is not necessarily due to detrusor overactivity (DO) ${ }^{21}$. In order to assess the role of perception in overactive bladder, we compared self-awareness and self-consciousness, combined with sensation related bladder diaries (SR-BD), in healthy volunteers and patients with OAB. The questionnaire used to asses self-awareness, the Situational Self-Awareness Scale (SSAS) ${ }^{22}$ is more sensitive to situational changes than the questionnaire which was used to asses self-consciousness, the Self-Consciousness Scale (SCS) ${ }^{23}$. Self-focus, which is the ability of a person to direct conscious attention on themselves, thoughts and feelings, can be dispositional or situational. Dispositional self-focus has often been referred to as "selfconsciousness," whereas situational self-focus was labelled as "self-awareness" 24.

We identified significant differences in bladder awareness between healthy volunteers and patients with OAB. Self-awareness and self-consciousness related to voiding, was analysed, and a difference in self-awareness was noted. Patients were more aware 
of their bladder and subsequent feelings as compared to volunteers. In the literature, an association has been suggested between the mental and the bodily state ${ }^{25}$. Furthermore, the ability to notice visceral responses differently will influence "measures of subjective affective experience". It is well known that detection of internal symptoms, such as heart rate or bladder sensations, is influenced by psychological processes, and that these processes can explain why people are not always good at interpreting internal physiological activity validly ${ }^{26}$. In addition, psychiatric conditions, especially mood and anxiety disorders, are characterized by enhanced somatic symptoms and increased attention to internal autonomic processes ${ }^{27}$.

In this thesis we hypothesise that patients with $O A B$ are more sensitive to bladder sensations. As in our study of bladder awareness, patients with OAB had lower volumes per void with higher urgency scores compared to healthy volunteers. Howsoever, the bladder fullness perception revealed no significant differences between the group. Thus, bladder sensations are labelled differently. An integrated sensitization or alarm falsification process might be involved, bringing together peripheral signals and the central amplification to result in OAB. A recent case control study reported significantly higher psychological stress levels in patients with OAB-wet and in patients with bladder pain syndrome (BPS), compared with healthy controls. A positive correlation between perceived stress levels and urinary incontinence symptoms was noted among OAB patients ${ }^{28}$. Furthermore, results from a health survey indicated that women with major depression perceived their incontinence symptoms as significantly more severe than non-depressed women ${ }^{29}$.

Obvious overlap exists between functional conditions such as bladder pain syndrome (BPS) and OAB. Approximately one third of OAB patients reported symptoms that are classically associated with BPS, while nearly half of the patients with BPS reported urgency incontinence in a prospective study. These findings suggest a hypothesis of one bladder hypersensitivity syndrome, with BPS on one end, and OAB at the other end of the same spectrum ${ }^{30}$. Similarly, BPS, and other functional disorders such as irritable bowel syndrome (IBS) or fibromyalgia, is often interrelated, which implicates that a general or common hypersensitivity may exist ${ }^{31}$. In this light the results of a pilot study of 7 women with predominantly urgency urinary incontinence (UUI) who enrolled in a 8 week Mindfulness-based stress reduction (MBSR) programme are interesting ${ }^{32}$. Voiding diaries learned that incontinence episodes were significantly reduced after treatment ${ }^{32}$. Mindfulness-based stress reduction (MBSR) is a mind-body intervention that incorporates mindfulness techniques in a group interventional cognitive behavioural framework. Mindfulness literally means paying attention on a purpose, in the present moment and nonjudgmentally ${ }^{33}$. Encouraged by these results the same group conducted a randomized trial of treatment with either yoga or MBSR. Thirty women with predominantly UUI enrolled in this 8 week programme, without receiving other additional treatments. Significantly more reduction in the UUI episodes was shown in the MBSR group at 8 weeks and 6 months compared to the yoga group ${ }^{34}$. The explana- 
tion of the authors regarding working mechanisms of MBSR are literally: " to teach participants skills that promotes non-judgmental awareness of body sensations, to interpret body sensations as simply sensations, as opposed to interpretations and thoughts about the sensations (e.g., catastrophizing). Mindfulness skills may allow participants to actually change their brain response to urgency by interpreting the sensation differently (i.e. nonjudgmentally)." Mindfulness based therapies have been proven to be beneficial in other functional disorders as well, like, for instance, IBS ${ }^{35}$.

Change of perception of symptoms was also seen by Tadic, who assessed the effect of biofeedback on the psychological burden and symptoms of OAB wet ${ }^{36}$. Biofeedback and behavioural therapy was applied, as a mind-body intervention, in which subjects were taught how to control urgency themselves. The aim was to improve UI and psychological burden. Although the benefit concerning UI frequency was similar between subjects with and without a history of depression, improvement in psychological burden was twice as large in subjects with a history of depression. It was found that the psychological change was related to improved perception of disease control. Therefore the regaining of control might result in a greater improvement with regard to psychological burden.

In our study, a difference was shown in awareness and perception of urgency between $O A B$ patients and healthy volunteers. If it would be possible to distinguish between $O A B$ due to merely bladder causes, and $O A B$ due to the perception of urgency, this may have implications for therapy. Then the first category would have to be treated with the established therapeutic options, while the second category of patients would have to be treated in a different way. This would also mean that integrated care should be given. Possible treatment options would be MBSR, biofeedback, and cognitive behavioural treatment or even the application of centrally working medications (e.g. antidepressants).

\section{PSYCHOSOMATIC PARAMETERS RELATED TO URODYNAMIC RESULTS}

In addition, we compared psychometric parameters with urodynamic results in a population with increased urinary frequency. Although numerous articles about $O A B$ and affective symptoms have been published, studies combining urodynamic data with anxiety and depression scores are scarce. Increased urinary frequency is considered one of the most bothersome symptoms of $O A B{ }^{37}$. Furthermore, $O A B$ is a diagnosis based on patients' symptoms, and $D O$ is a urodynamic finding ${ }^{38}$. A distinction can be made between two groups of patients with regard to DO: OAB patients with DO and OAB patients without DO, with the first group experiencing worse symptoms ${ }^{39}$. The study described in this thesis revealed a significant association between the total HADS scores and DO, between HADS anxiety scores and DO, and between HADS depression scores and the feeling of urgency. The sparse literature available on this subject, showed high- 
er depression and anxiety rates in urodynamically proven stress urinary incontinence (SUI), DO or sensory urgency compared to normal controls. However, among these diagnostic categories no differences were reported with respect to affective complaints 40. In two other studies there was no relation between affective symptoms and urodynamic diagnosis ${ }^{41,42}$. The association between UUI, proven by urodynamic study (UDS), and depression has been described but the association between DO and affective disorders has not been described before ${ }^{43,44}$. It has been noted that patients with DO have lower quality of life scores, and a lower mental health domain score on the SF 36 compared to patients without DO ${ }^{45}$. In our study, increased urinary frequency was not associated with DO on conventional-UDS. This is consistent with earlier reported results, in which frequency alone has been proven to be a poor predictor of DO ${ }^{21}$. Nevertheless, it has been hypothesised that increased frequency of micturition originates from cognitive strategies to avoid intense urgency and incontinence ${ }^{46}$. A correlation between $\mathrm{OAB}$ symptoms and urodynamic diagnoses has been described by Hashim and colleagues: DO was present in $69 \%$ of the men and $44 \%$ of women with OAB dry, and $90 \%$ and $44 \%$ in $O A B$ wet ${ }^{21}$. We did not detect an association between the complaints of the patients or parameters of the voiding diaries and anxiety or depression, but an association was found between HADS scores and DO. The total HADS score was significantly associated with the presence of DO in women but not in men. Similarly, a positive association between HADS anxiety and DO was detected in women but not in men. No association was found between HADS depression and DO, however a significant association between HADS depression and the sensation of urgency was revealed. A possible explanation for the differences between men and women could be that the nature of the DO is different, as DO in men is more often due to an anatomical obstruction at the level of the prostatic urethra and/or the bladder neck.

\section{SCREENING FOR AFFECTIVE DISORDERS}

Sacral neuromodulation (SNM) is an advanced treatment option for LUTD. However, treatment failure rate ranges between $31 \%$ and $54 \%$, depending on the initial selection of patients ${ }^{47}$. It has been proven to be difficult to define reliable predictive factors to identify patients which are more likely to benefit from SNM ${ }^{48,49}$. In this thesis we assessed whether the presence of affective symptoms is anyhow related to treatment outcome. The results of our study indicated that affective comorbidity, as screened with the HADS, was not a predictive factor for the treatment outcome of SNM in patients with LUTD. Higher HADS scores neither predicted failure in the initial test period, nor the loss of treatment effect after one year. However, several meaningful associations between HADS score and LUTD were noted. First, a third of the patients had a psychiatric history, and a significant association between HADS scores and the presence of psychiatric history was seen. This is an affirmation of the external validity of the HADS 
questionnaire, which indicates that the HADS questionnaire may be used validly in patients with functional LUTD. Secondly, in our study, patients with OAB have a 3.8 (odds ratio) times higher chance of having an abnormal HADS score, compared to the patients with non-obstructive retention (NOR). This indicates that OAB may be more influenced by pathways of the brain bladder axis than NOR. Thirdly, an abnormal HADS-score was seen significantly more often in OAB-wet patients, compared to OAB-dry patients, which is in accordance with the literature. Other studies reported that quality of life is also reduced to a larger extent in patients with OAB-wet, compared to patients with OAB-dry ${ }^{50}$.

However, our presented study should be seen in the light of the limitation of selection bias, as the indication for SNM was made before entering the study. Our pelvic care centre delivers integrated care, which may have led to a positive selection bias in the referral for SNM. This might be reflected by the fact that within our group of patients almost 70\% had normal anxiety and depression scores, indicating no affective disorder at the moment of SNM. Thus, the value of preselection in a "normal" practice cannot be addressed by the outcome of this study, as no randomized treatment selection existed. Therefore, although we did not report a significant relation between an elevated HADS score and success or failure of neuromodulation, we still would advocate the necessity for psychiatric screening in patients with LUTD.

Anxiety and depression are prevalent, and often underdiagnosed disorders, which influences prognosis of somatic conditions in general, as well as health care consumption in somatic conditions ${ }^{51-55}$. For example, in a longitudinal study, depression at baseline was associated with the persistence of urinary incontinence over 5 years in women ${ }^{56}$. In turn, anxiety and depressive symptoms can intensify UI and decrease the benefit of treatment ${ }^{57}$. Underlying psychiatric pathways might be involved, since patients with neuroticism were less likely to respond to treatment of DO compared to patients without ${ }^{58}$. Nevertheless, Marcelissen et al. found no evidence that psychological screening for personality traits and psychological status could predict success with SNM in patients with chronic LUTS ${ }^{59}$. On the other hand, patients with a history of psychiatric conditions, experienced more often adverse events during permanent SNM treatment 59. Additionally, a multicentre evaluation of SNM indicated that psychological disturbances were present in 70\% of patients with LUTD. Moreover, the test stimulation was less reliable in patients with a psychiatric history ${ }^{60}$.

A large population based survey showed an inverse relationship between depressive symptoms and treatment seeking for bladder dysfunctions. Depressive symptoms can thus hinder patients from seeking treatment ${ }^{61}$. It is possible that there is a reinforcing cycle of increasing symptoms of $\mathrm{OAB}$, with increasing bother and enhanced affective symptoms without treatment seeking. Compared to non-depressed patients, the odds are three times higher for depressed patients to be non-compliant to an advised medical treatment. This poses a possible risk factor for poor treatment outcome, since patients might not adhere to the medical directives that were provided ${ }^{62}$. 
Hence, we recommend that urologists should use a validated questionnaire to screen for affective conditions in all patients with LUTD who are eligible for treatment. This recommendation makes sense since a depression can be effectively treated in the case of a medical comorbidity, and recovery rates of depression can be achieved, comparable to those of patients with depression without a comorbid medical illness ${ }^{63}$. Furthermore, having insight into patients' emotional state may improve communication and prevent possible patient dissatisfaction. By recognizing and treating possible underlying affective conditions, the response to medical management for LUTS might be improved. Since our multidisciplinary triage system could also result in a psychiatric consultation, this integrated approach may have influenced the outcome of our study positively. Thus, SNM should not be withheld to patients with affective conditions, and should be offered within an integrated approach of care.

\section{PATIENT REPORTED OUTCOME}

Patient reported outcome (PRO) is a measurement of a patient's health status that is provided directly by the patient, and delivers evidence of treatment benefit from the patients' perspective ${ }^{64}$. PRO has gained increasing attention both in clinical research and in treatment evaluation by health care insurances and regulatory agencies. In the United Kingdom (UK), routine collection of patient-reported outcome measures (PROMs) was used to measure the performance of health-care providers ${ }^{65}$. Furthermore, evaluation of patient related outcome can guide in a change of treatment decision ${ }^{66}$.

We collected PRO with a structured interview by telephone, which was conducted in patients with UI seen in our multidisciplinary academic pelvic care clinic (PCC) at one year after the end of treatment. The results of the telephone survey were compared with the complaints reported during the triage at the beginning of treatment in our centre. Patients reported a significant improvement of mean severity of incontinence at one year after the end of treatment as compared to the pre-treatment situation. One out of three patients went from having a multifactorial health problem to a monofactorial health problem. Additionally, patients claimed high satisfaction with received care. There was a remarkable difference between patient-reported cure $(20.6 \%)$ and patients who no longer required pads for UI (26.6\%). So even without requiring pads some patients do not consider themselves cured, which can possibly be explained by a lack of trust in their own body.

PRO is a complex measure that can be hard to objectively assess or interpret. Subjective improvement of treatment satisfaction often does not correlate with the objective improvement in pad use or outcomes of urodynamic investigations ${ }^{67,68}$. Patient satisfaction seems to be dependent on many different factors, such as patient's age, patient-physician interaction, influence of the complaints on daily activities and patient's expectations ${ }^{69}$. In addition, PRO can be influenced by anxiety or depression, as 
seen in a cognitive rehabilitation programme in patients with chronic musculoskeletal pain ${ }^{70}$. The programme yielded a better outcome for PROMs in younger subjects with higher depression scores at baseline. Depressive symptoms and anxiety had a negative impact on self-assessed outcome measurements in patients scheduled for rotator cuff repair $^{71}$. In orthopaedic surgery, preoperative psychological distress, such as depressive symptoms and anxiety, was associated with poor self-reported clinical outcome after surgery ${ }^{72,73}$. In patients with chronic pelvic pain syndrome (CPPS), depression is associated with the reported severity of pain, therefore depression influences the perception of complaints ${ }^{74}$.

In our study we noted that patients suffering from burn-out, depression or anxiety, as based on medical records, or self-reported data before treatment, scored higher on mean severity of UI at baseline, compared to patients without a psychological or psychiatric history. However, these psychological factors had no influence on the reported UI one year after the end of treatment.

There still is considerable heterogeneity in defining treatment response, with respect to success or failure rate, by health care professionals and patients with LUTD. PRO as a tool for the evaluation of the effect of therapy is estimated to be highly useful in clinical practice and may therefore provide important information ${ }^{75}$. Overall, this raises the question as to whether $\mathrm{PRO}$, as a tool, is reliable enough to rely on, especially in a population with a high incidence of affective disorders.

\section{THE ASSOCIATION OF AFFECTIVE DISORDERS AND LUTD}

The interrelationship between psychological factors and functional gastrointestinal disorders such as IBS or functional dyspepsia, is well known, and described along the "gut-brain-axis" and functional problems are thought to be a result of a disturbed regulation along that axis. In the pathogenesis of IBS the association between somatic and psychiatric factors is proven to be bi-directional ${ }^{76}$. LUTD can potentially also be explained along a similar "bladder-brain-axis" or even "bladder-gut-brain-axis". Along that axis, three causative mechanisms can be hypothesised. First, LUTD can cause the onset of depression and anxiety disorders ${ }^{77,78}$. Since, LUTD can lead to accumulation of stress due to social and functional impairment and in turn, may lead to affective states ${ }^{29}$. Second, depression and anxiety can predict the onset of LUTD ${ }^{77-80}$. Third, affective disorders and LUTD both share common biological pathways.

Affective disorders and LUTD may indeed share common biological pathways, and some data support this hypothesis. Serotonergic and noradrenergic neurotransmitters, as well as the complex interactions between these two neurotransmitters, are thought to play a role in the aetiology of depression and anxiety disorders ${ }^{81}$. There seems to be an overall increased noradrenergic activity, and an overall decreased activity of the serotonergic system in affective disorders ${ }^{81}$. And serotonin depletion can contribute to 
depression, and selective serotonin reuptake inhibitors (SSRIs) are effective in depression treatment ${ }^{82}$. Moreover, in anxiety disorders, it is likely that the serotonergic system plays a role in the regulation of anxiety, both during development and in adulthood ${ }^{83}$. Serotonin also plays a role in bladder function at a central level. Several animal studies revealed that lowering serotonin levels in the central nervous system (CNS) was accompanied by increased urinary frequency and DO ${ }^{84-86}$. Experimental studies in rats and cats indicated that activation of the central serotonergic system depresses reflex bladder contractions, and increases the bladder volume threshold for micturition ${ }^{87}$. Affective disorders may thus be linked to $O A B$, due to altered serotonin function. Moreover, in a rat model of depression, a lowering of serotonin levels in the CNS, produced altered bladder function such as increased urinary frequency due to non-neurogenic DO, and lowered bladder capacity in female rats. Administration of a selective serotonin reuptake inhibitor (SSRI) reversed urinary symptoms ${ }^{88}$. In humans, duloxetine, a serotonin-noradrenaline reuptake inhibitor (SNRI) can be used for LUTD and affective disorders alike. This drug inhibits the re-uptake of the neurotransmitters serotonin (5-HT) and norepinephrine (NE). The increased concentrations of both 5-HT and NE leads to increased stimulation of the pudendal nerve, and therefore increases the resting tone and contraction of the urethral sphincter ${ }^{89}$. Initially, it was solely researched for the treatment of stress urinary incontinence (SUI). A systematic review regarding the use of duloxetine in SUI reported a cure rate of around 50\%. However, the follow up was short, and considerable side effects were noted ${ }^{90}$. In the Netherlands, duloxetine is only registered as an antidepressant and not for use in SUI, such as in the UK ${ }^{91}$. Given that also effects on $O A B$ symptoms were noted, this was the subject of another clinical trial. A 12-week, randomized, placebo-controlled, double-blind clinical trial including 306 women with $O A B$ revealed that duloxetine was better than placebo in the treatment of OAB dry and wet ${ }^{92}$. The total voiding episodes per 24 hours decreased with 1.9 and the UUI episodes decreased with $0.87^{92}$. The clinical value of a new approach for the treatment of $O A B$ needs to be confirmed by a study comparing SNRI's with standard treatment of $O A B$.

Another possibility with regard to common pathways in LUTD and affective disorders, may be related to a shared dysregulation of the hypothalamic-pituitary-adrenal (HPA) axis. This axis is connected to the limbic system, and plays an important role in stress regulation and management. The HPA axis mediates a bi-directional brain body communication during exposure to aversive stimuli, and regulates the individual adaptive response to threat (i.e. alarm reaction) ${ }^{93}$. Dysregulation results in increased corticotroponin-releasing factor (CRF), increased adrenocorticotropic hormone (ACTH), and increased cortisol release. Dysregulation within the HPA-axis is thought to play a role in depression and anxiety disorders ${ }^{82,94,95}$. And there is increasing evidence that CRF is involved in bladder function as well ${ }^{96-98}$.

In addition, a functional overlap exists in different anatomic parts of the (central) nervous system, such as the brainstem and the spinal cord. Both are involved in HPA-axis 
mediated stress response, as well as in the control of voiding. Acute and chronic foot shock stress in rats resulted in enhanced CRF mRNA expression in Barrington's nucleus, the brainstem region that controls micturition ${ }^{99}$. Additionally, in rodents, CRF is present in a descending projection to the spinal cord that may be involved in regulating the micturition reflex. This indicates that CRF is also involved in the control of micturition at the level of the spinal cord. Repeated psychological distress in rats resulted in increased urinary frequency and lower voiding volumes ${ }^{100}$. Furthermore, CRF stimulated bladder function (lowered micturition threshold and lower urine volume) in a rodent model of DO and anxiety. Administration of a CRF antagonist reduced DO ${ }^{101}$. Another study with repeated social stress in rats, revealed a urodynamic pattern that resembled urodynamic signs of overactive detrusor. This abnormal pattern, was observed 3-4 days after the last exposure to stress, which may be suggestive of an enduring or latent effect ${ }^{102}$.

In this thesis we did not use any biomarkers or specific questionnaire to assess stress, but in patients with increased urinary frequency we saw a significant association between total HADS scores and the presence of DO during urodynamic investigation. We speculate that a possible explanation for this could be given along the aforementioned dysregulation of the HPA axis. In addition, the association between OAB and affective disorders, as described in this thesis, may also be explained by a common dysregulation of the involved serotonergic and noradrenergic neurotransmitter systems. Future research should elaborate the exact role of these possibilities.

\section{ANATOMICAL OVERLAP IN LUTD AND AFFECTIVE DISORDERS.}

Convincing evidence from functional brain magnetic resonance imaging (fMRI) during urodynamic studies showed that increased brain activation in the anterior cingulate gyrus (ACG), which is a part of the anterior cingulate cortex (ACC), was associated with increased urgency at large bladder volumes. This was especially seen in young females with UI and DO ${ }^{103-105}$. In young people with a depressive disorder increased activity levels were found in the ACG as well ${ }^{106}$. The ACC, is part of a neural stress-network, which is involved in (social) pain aspects ${ }^{107,108}$. Moreover, single photon emission computed tomography (SPECT) of older patients with UUI showed hypo-perfusion in the ACG and other frontal lobe regions such as the prefrontal cortex ${ }^{109}$. Similarly, in patients with a major depressive disorder, hypo-perfusion in the ACC and the prefrontal cortex was also seen ${ }^{110}$. Damage at the level of the right superior frontal cortex has been associated with transient incontinence and bilateral damage with permanent incontinence ${ }^{111}$. Comparably, thinning of the cortical mantle in the right frontal cortex has been described in individuals who are at increased familial risk for depression ${ }^{112}$.

Overlap between psychiatric conditions and voiding dysfunction can also be seen in childhood enuresis. Enuresis was found to be a "premorbid developmental marker for schizophrenia" ${ }^{113}$. Patients with enuresis scored worse on two frontal lobe tests, com- 
pared to patients without enuresis. The associated cerebral defects were around the ACG and the prefrontal cortex, similar to patients with UUI ${ }^{113}$. Similarly, abnormal ACC activation was seen in adults with IBS as well, indicating that the "gut-brain axis" and the "bladder-brain-axis" may, at least in part, be a shared axis ${ }^{114}$.

These findings suggest that prefrontal brain regions and parts of the ACC may mediate both affective disorders and functional somatic complaints (OAB). Present knowledge is that the ACC is also involved in attention (e.g. bladder filling and rectal distension), and executive control (appropriate timing of voiding). Differences in functionality of the ACC might be an explanation for the differences in self- awareness between patients with $O A B$ and healthy volunteers, which has been elaborated on in this thesis.

\section{FUTURE PERSPECTIVES}

In this thesis we hypothesised that there is a common phenotype and pathway, which explains associations of LUTD with affective symptoms. Additionally, we suggested that patients with $O A B$ respond differently to normal bladder sensations. Future investigations should explore possible underlying common pathways while elaborating on different aspects of bladder sensation. From a clinical point of view, the association of LUTD and affective symptoms should be confirmed in a prospective study to further clarify the direction of causality, concerning LUTD and psychological factors.

To determine the role of stress on bladder function in humans prospectively, will be challenging but very necessary. A possible field of research could be related to the differences in stress reaction between healthy volunteers and patients with $O A B$ as hormones along the hypothalamic-pituitary-adrenal (HPA) axis can be tested in humans. Since cortisol response, together with voiding parameters, can be measured under (artificially) stressful circumstances, it is possible to obtain results on this system in individuals with and without LUTD.

Based on our results we speculate that patients with $O A B$ respond differently to normal bladder sensations, and are generally more sensitive to bodily signals. A possible neural focus could be the Anterior Insula Cortex, where signals are registered and labelled with emotional meanings. Future studies should aim at localizing further regions known to be involved in affective conditions, and determine their exact function. Functional MRI studies during urodynamic investigation may be able to expand our knowledge regarding this matter.

From a clinical point of view, we appeal for multidisciplinary integrated care in patients with treatment-resistant $O A B$ and other forms of LUTD. Active screening for affective disorders should be mandatory, in order to obtain better integrated treatment results, lower health care costs and a better quality of life. The results of a pilot study of integrated medical-psychiatric outpatients care in functional gastrointestinal problems 
are very encouraging, and give an example of a multimodal approach in functional disorders. Integrated care resulted in significant improvement of gastrointestinal and psychiatric symptoms, as well as quality of life, in 124 patients with complex functional gastrointestinal disorders compared to care as usual ${ }^{115}$. In the future we will conduct a similar study for the functional urological patient population.

Most of all, there is a need for an adequate assessment of symptom screening in general that overcomes the limitations of currently available methods. The current methods rely on diaries and retrospective questionnaires, which are influenced by recall bias and circumstantial and psychological factors. These limitations might be overcome by randomly, repeated momentary assessment during the day in a real life environment. This Experience Sampling Methodology (ESM) research method asks subjects to stop at certain times and make notes of their experience in real time by asking specific questions, usually done through a mobile app. ESM revealed already that IBS patients reported higher scores for pain in retrospective questionnaires compared to ESM. The patients tended to report peak scores rather than the average pain scores ${ }^{116}$. Hence, ESM may provide a better overview of the course of symptoms, and indicate potential triggers that may influence symptom development. It is of further importance that momentary assessment in daily life can combine symptoms of physical and psychological origin instantly, and may lead to a better understanding of functional symptomatology. It is foreseeable that a future development of ESM will incorporate psychological and physical symptom assessment with statistical network methodology. This then will reveal more realistic symptom clusters, which are usually overlooked from a nowadays mono-disciplinary point of view. In the future ESM could be used to select patients with LUTD who may benefit from psychological treatment. Therefore, it is very encouraging that our multidisciplinary research project regarding "ESM in complex gastrointestinal and urological disorders; development and validation of a real-time and real-life patient-reported outcome measure for somatic and psychological symptoms" is rewarded with a substantial grant. This future research project will enable us to explore the possibilities of ESM in patients with bladder complaints. For sure, ESM will be part of personalized medicine and will be used as a diagnostic and treatment tool incorporating cognitive behavioural aspects of management of LUTD.

The consideration that mental factors can be a possible cause for bladder dysfunction may enable us, as urologists, to improve diagnosis and to identify patients who will benefit from a multimodal treatment. For this approach it is not needed to become an "uropsychiatrist" but it will allow any clinician involved in diagnosis and treatment of LUTD to apply a more holistic approach that will be beneficial to patients, society and even to caregivers themselves. 


\section{REFERENCES}

1. Felde, G., M.H. Ebbesen, and S. Hunskaar, Anxiety and depression associated with urinary incontinence. A 10-year follow-up study from the Norwegian HUNT study (EPINCONT). Neurourol Urodyn, 2017.36(2): p.322-328.

2. Mishra, G.D., et al., Depression and the incidence of urinary incontinence symptoms among young women: Results from a prospective cohort study. Maturitas, 2015. 81(4): p. 456-61.

3. Townsend, M.K., et al., Urinary incontinence and prevalence of high depressive symptoms in older black versus white women. Int Urogynecol J, 2014. 25(6): p. 823-9.

4. Lai, H.H., et al., The relationship between depression and overactive bladder/urinary incontinence symptoms in the clinical OAB population. BMC Urol, 2016. 16(1): p. 60.

5. Torske, M.O., et al., Farmers' mental health: A longitudinal sibling comparison - the HUNT study, Norway. Scand J Work Environ Health, 2016. 42(6): p. 547-556.

6. Cardoso, G., et al., Depression and anxiety symptoms following cancer diagnosis: a cross-sectional study. Psychol Health Med, 2015: p. 1-9.

7. Ng, T.P., et al., Depressive symptoms and chronic obstructive pulmonary disease: effect on mortality, hospital readmission, symptom burden, functional status, and quality of life. Arch Intern Med, 2007. 167(1): p. 60-7.

8. Hellstadius, Y., et al., Prevalence and predictors of anxiety and depression among esophageal cancer patients prior to surgery. Dis Esophagus, 2016. 29(8):1128-1134.

9. Iyasere, O.U., et al., Quality of Life and Physical Function in Older Patients on Dialysis: A Comparison of Assisted Peritoneal Dialysis with Hemodialysis. Clin J Am Soc Nephrol, 2016. 11(3):p.423-30

10. Williams, J.B., et al., Preoperative anxiety as a predictor of mortality and major morbidity in patients aged >70 years undergoing cardiac surgery. Am J Cardiol, 2013. 111(1): p. 137-42.

11. Ghetti, C., et al., Depressive symptoms in women seeking surgery for pelvic organ prolapse. Int Urogynecol J, 2010. 21(7): p. 855-60.

12. Zeleke, B.M., et al., Depression among women with obstetric fistula, and pelvic organ prolapse in northwest Ethiopia. BMC Psychiatry, 2013. 13: p. 236.

13. Merrell, J., et al., Psychosocial correlates of pelvic floor disorders in women seeking bariatric surgery. Surg Obes Relat Dis, 2012. 8(6): p. 792-6.

14. Ghetti, C., et al., The Emotional Burden of Pelvic Organ Prolapse in Women Seeking Treatment: A Qualitative Study. Female Pelvic Med Reconstr Surg, 2015. 21(6): p. 332-8.

15. Iversen, M.M., et al., Anxiety and Depressive Symptoms as Predictors of All-Cause Mortality among People with Insulin-Naive Type 2 Diabetes: 17-Year Follow-Up of the Second Nord-Trondelag Health Survey (HUNT2), Norway. PLoS One, 2016. 11(8): p. e0160861.

16. Woolrich, R.A., P. Kennedy, and T. Tasiemski, A preliminary psychometric evaluation of the Hospital Anxiety and Depression Scale (HADS) in 963 people living with a spinal cord injury. Psychol Health Med, 2006. 11(1): p. 80-90.

17. Geirdal, A.O., et al., Psychological distress in women at risk of hereditary breast/ovarian or HNPCC cancers in the absence of demonstrated mutations. Fam Cancer, 2005. 4(2): p. 121-6.

18. Gu, G., et al., Increased prevalence of anxiety and depression symptoms in patients with coronary artery disease before and after percutaneous coronary intervention treatment. BMC Psychiatry, 2016. 16: p. 259.

19. Wyndaele, J.J. and S. De Wachter, Cystometrical sensory data from a normal population: comparison of two groups of young healthy volunteers examined with 5 years interval. Eur Urol, 2002. 42(1): p. 34-8.

20. De Wachter, S.G., et al., On the nature of bladder sensation: the concept of sensory modulation. Neurourol Urodyn, 2011. 30(7): p. 1220-6.

21. Hashim, H. and P. Abrams, Is the bladder a reliable witness for predicting detrusor overactivity? J Urol, 2006. 175(1): p. 191-4; discussion 194-5. 
22. Govern, J.M. and L.A. Marsch, Development and validation of the situational self-awareness scale. Conscious Cogn, 2001. 10(3): p. 366-78.

23. Vleeming, R.G. and J.A. Engelse, Assessment of Private and Public Self-Consciousness: A Dutch Replication. Journal of Personality Assessment, 1981. 45(4): p. 385.

24. Fenigstein, A., M.F. Scheier, and A.H. Buss, Public and private self-consiousness: Assessment and theory. Journal of Consulting and Clinical Psychology, 1975. 43: p. 522-7.

25. Cameron, O.G., Interoception: the inside story--a model for psychosomatic processes. Psychosom Med, 2001. 63(5): p. 697-710.

26. Pennebaker, J.W., Psychological bases of symptom reporting: perceptual and emotional aspects of chemical sensitivity. Toxicol Ind Health, 1994. 10(4-5): p. 497-511.

27. Mumford, D.B., et al., The Bradford Somatic Inventory. A multi-ethnic inventory of somatic symptoms reported by anxious and depressed patients in Britain and the Indo-Pakistan subcontinent. Br J Psychiatry, 1991. 158: p. 379-86.

28. Lai, H., et al., Correlation between psychological stress levels and the severity of overactive bladder symptoms. BMC Urol, 2015. 15: p. 14.

29. Melville, J.L., et al., Incontinence severity and major depression in incontinent women. Obstet Gynecol, 2005. 106(3): p. 585-92.

30. Lai, H.H., et al., The overlap and distinction of self-reported symptoms between interstitial cystitis/bladder pain syndrome and overactive bladder: a questionnaire based analysis. J Urol, 2014. 192(6): p. 1679-85.

31. Fink, P. and A. Schroder, One single diagnosis, bodily distress syndrome, succeeded to capture 10 diagnostic categories of functional somatic syndromes and somatoform disorders. J Psychosom Res, 2010. 68(5): p. 415-26.

32. Baker, J., D. Costa, and I. Nygaard, Mindfulness-based stress reduction for treatment of urinary urge incontinence: a pilot study. Female Pelvic Med Reconstr Surg, 2012. 18(1): p. 46-9.

33. Kabat-Zinn, J., Full catastrophe living: using the wisdom of your body and mind to face stress, pain and illness. 2005, New York: Delta.

34. Baker, J., et al., Comparison of mindfulness-based stress reduction versus yoga on urinary urge incontinence: a randomized pilot study. with 6-month and 1-year follow-up visits. Female Pelvic Med Reconstr Surg, 2014. 20(3): p. 141-6.

35. Aucoin, M., M.J. Lalonde-Parsi, and K. Cooley, Mindfulness-based therapies in the treatment of functional gastrointestinal disorders: a meta-analysis. Evid Based Complement Alternat Med, 2014. 2014: p. 140724.

36. Tadic, S.D., et al., Effect of biofeedback on psychological burden and symptoms in older women with urge urinary incontinence. Journal of the American Geriatrics Society, 2007. 55(12): p. 2010-2015.

37. Elinoff, V., et al., Symptom-specific efficacy of tolterodine extended release in patients with overactive bladder: the IMPACT trial. Int J Clin Pract, 2006. 60(6): p. 745-51.

38. Haylen, B.T., et al., An International Urogynecological Association (IUGA)/International Continence Society (ICS) joint report on the terminology for female pelvic floor dysfunction. Neurourol Urodyn, 2010. 29(1): p. 4-20.

39. Guralnick, M.L., et al., Objective differences between overactive bladder patients with and without urodynamically proven detrusor overactivity. Int Urogynecol J, 2010. 21(3): p. 325-9.

40. Macaulay, A.J., R.S. Stern, and S.L. Stanton, Psychological aspects of 211 female patients attending a urodynamic unit. J Psychosom Res, 1991. 35(1): p. 1-10.

41. Chiara, G., et al., Psychological investigation in female patients suffering from urinary incontinence. Int Urogynecol J Pelvic Floor Dysfunct, 1998. 9(2): p. 73-7.

42. Lagro-Janssen, A.L., F.M. Debruyne, and C. Van Weel, Psychological aspects of female urinary incontinence in general practice. Br J Urol, 1992. 70(5): p. 499-502.

43. Zorn, B.H., et al., Urinary incontinence and depression. J Urol, 1999. 162(1): p. 82-4.

44. Stach-Lempinen, B., et al., Severe depression determines quality of life in urinary incontinent women. Neurourol Urodyn, 2003. 22(6): p. 563-8. 


\section{Chapter 8}

45. Duggan, P., Urodynamic diagnoses and quality of life in women presenting for evaluation of urinary incontinence. Aust N Z J Obstet Gynaecol, 2011. 51(5): p. 416-20.

46. Harvey, J., et al., The relationship between cognition and sensation in determining when and where to void: the concept of cognitive voiding. BJU Int, 2012. 110(11): p. 1756-61.

47. Marcelissen, T., et al., Is the screening method of sacral neuromodulation a prognostic factor for longterm success? J Urol, 2011. 185(2): p. 583-7.

48. Scheepens, W.A., et al., Predictive factors for sacral neuromodulation in chronic lower urinary tract dysfunction. Urology, 2002. 60(4): p. 598-602.

49. Koldewijn, E.L., et al., Predictors of success with neuromodulation in lower urinary tract dysfunction: results of trial stimulation in 100 patients. J Urol, 1994. 152(6 Pt 1): p. 2071-5.

50. Hashim, H. and P. Abrams, Overactive bladder: an update. Curr Opin Urol, 2007. 17(4): p. 231-6.

51. Kroenke, K., Patients presenting with somatic complaints: epidemiology, psychiatric comorbidity and management. Int J Methods Psychiatr Res, 2003. 12(1): p. 34-43.

52. Kessler, R.C., et al., Lifetime prevalence and age-of-onset distributions of DSM-IV disorders in the National Comorbidity Survey Replication. Arch Gen Psychiatry, 2005. 62(6): p. 593-602.

53. Demyttenaere, K., et al., Prevalence, severity, and unmet need for treatment of mental disorders in the World Health Organization World Mental Health Surveys. JAMA, 2004. 291(21): p. 2581-90.

54. Penninx, B.W., et al., Depression and cardiac mortality: results from a community-based longitudinal study. Arch Gen Psychiatry, 2001. 58(3): p. 221-7.

55. Roy-Byrne, P.P., et al., Anxiety disorders and comorbid medical illness. Gen Hosp Psychiatry, 2008. 30(3): p. 208-25.

56. Maserejian, N.N., et al., Treatment status and risk factors for incidence and persistence of urinary incontinence in women. Int Urogynecol J, 2014. 25(6): p. 775-82.

57. Bogner, H.R., et al., Anxiety disorders and disability secondary to urinary incontinence among adults over age 50. Int J Psychiatry Med, 2002. 32(2): p. 141-54.

58. Moore, K.H. and J.R. Sutherst, Response to treatment of detrusor instability in relation to psychoneurotic status. Br J Urol, 1990. 66(5): p. 486-90.

59. Marcelissen, T.A., et al., Psychological and psychiatric factors as predictors for success in sacral neuromodulation treatment. BJU Int, 2011. 108(11): p. 1834-8.

60. Everaert, K., et al., Patient satisfaction and complications following sacral nerve stimulation for urinary retention, urge incontinence and perineal pain: a multicenter evaluation. Int Urogynecol J Pelvic Floor Dysfunct, 2000. 11(4): p. 231-5; discussion 236.

61. Coyne, K.S., et al., The impact of overactive bladder on mental health, work productivity and healthrelated quality of life in the UK and Sweden: results from EpiLUTS. BJU Int, 2011. 108(9): p. 1459-71.

62. DiMatteo, M.R., H.S. Lepper, and T.W. Croghan, Depression is a risk factor for noncompliance with medical treatment: meta-analysis of the effects of anxiety and depression on patient adherence. Arch Intern Med, 2000. 160(14): p. 2101-7.

63. Gill, D. and S. Hatcher, A systematic review of the treatment of depression with antidepressant drugs in patients who also have a physical illness. J Psychosom Res, 1999. 47(2): p. 131-43.

64. Padros, J., et al., Evaluation of a urinary incontinence unit for community-dwelling older adults in Barcelona: implementation and improvement of the perceived impact on daily life, frequency and severity of urinary incontinence. Z Gerontol Geriatr, 2008. 41(4): p. 291-7.

65. Health, U.S.D.o., et al., Guidance for industry: patient-reported outcome measures: use in medical product development to support labeling claims: draft guidance. Health Qual Life Outcomes, 2006. 4: p. 79.

66. Devlin, N.J., D. Parkin, and J. Browne, Patient-reported outcome measures in the NHS: new methods for analysing and reporting EQ-5D data. Health Econ, 2010. 19(8): p. 886-905.

67. Wolters, J.P., A.B. King, and D.E. Rapp, Satisfaction in patients undergoing concurrent pelvic floor surgery for stress urinary incontinence and pelvic organ prolapse. Female Pelvic Med Reconstr Surg, 2014. 20(1): p. 23-6.

68. Padmanabhan, P. and V.W. Nitti, Female stress urinary incontinence: how do patient and physician perspectives correlate in assessment of outcomes? Curr Opin Urol, 2006. 16(4): p. 212-8. 
69. Marschall-Kehrel, D., R.G. Roberts, and L. Brubaker, Patient-reported outcomes in overactive bladder: the influence of perception of condition and expectation for treatment benefit. Urology, 2006. 68(2 Suppl): $p$. 29-37.

70. Bremander, A.B., G. Holmstrom, and S. Bergman, Depression and age as predictors of patient-reported outcome in a multidisciplinary rehabilitation programme for chronic musculoskeletal pain. Musculoskeletal Care, 2011. 9(1): p. 41-8.

71. Cho, C.H., et al., The impact of depression and anxiety on self-assessed pain, disability, and quality of life in patients scheduled for rotator cuff repair. J Shoulder Elbow Surg, 2013. 22(9): p. 1160-6.

72. Ackerman, I.N., et al., Evaluating quality of life in hip and knee replacement: Psychometric properties of the World Health Organization Quality of Life short version instrument. Arthritis Rheum, 2006. 55(4): p. 583-90.

73. Howard, K.J., et al., Psychosocial profiles of indigent patients with severe osteoarthritis requiring arthroplasty. J Arthroplasty, 2011. 26(2): p. 244-9.

74. Kwon, J.K. and I.H. Chang, Pain, catastrophizing, and depression in chronic prostatitis/chronic pelvic pain syndrome. Int Neurourol J, 2013. 17(2): p. 48-58.

75. Goldman, H.B., et al., Defining response and non-response to treatment in patients with overactive bladder: a systematic review. Curr Med Res Opin, 2014. 30(3): p. 509-26.

76. Koloski, N.A., et al., The brain--gut pathway in functional gastrointestinal disorders is bidirectional: a 12year prospective population-based study. Gut, 2012. 61(9): p. 1284-90.

77. Perry, S., C.W. McGrother, and K. Turner, An investigation of the relationship between anxiety and depression and urge incontinence in women: Development of a psychological model. British Journal of Health Psychology, 2006. 11(3): p. 463-482.

78. van de Pol, G., et al., Is there an association between depressive and urinary symptoms during and after pregnancy? Int Urogynecol J Pelvic Floor Dysfunct, 2007. 18(12): p. 1409-15.

79. Nuotio, M., et al., Six-year follow-up and predictors of urgency-associated urinary incontinence and bowel symptoms among the oldest old: a population-based study. Arch Gerontol Geriatr, 2009. 49(2): p. e8590.

80. Hirayama, A., et al., Risk factors for new-onset overactive bladder in older subjects: results of the Fujiwara-kyo study. Urology, 2012. 80(1): p. 71-6.

81. Ressler, K.J. and C.B. Nemeroff, Role of serotonergic and noradrenergic systems in the pathophysiology of depression and anxiety disorders. Depress Anxiety, 2000. 12 Suppl 1: p. 2-19.

82. Nemeroff, C.B., The neurobiology of depression. Sci Am, 1998. 278(6): p. 42-9.

83. Gordon, J.A. and R. Hen, The serotonergic system and anxiety. Neuromolecular Med, 2004. 5(1): p. 2740.

84. Steers, W., Potential targets in the treatment of urinary incontinence. Reviews in urology, 2001. 3 Suppl 1: p. S19-26.

85. Sohn, U.D. and C.Y. Kim, Suppression of the rat micturition reflex by imipramine. J Auton Pharmacol, 1997. 17(1): p. 35-41.

86. Maggi, C.A. and A. Meli, Reserpine-induced detrusor hyperreflexia: an in vivo model for studying smooth muscle relaxants at urinary bladder level. J Pharmacol Methods, 1983. 10(2): p. 79-91.

87. de Groat, W.C., Influence of central serotonergic mechanisms on lower urinary tract function. Urology, 2002. 59(5 Suppl 1): p. 30-6.

88. Lee, K.S., et al., Alterations in voiding frequency and cystometry in the clomipramine induced model of endogenous depression and reversal with fluoxetine. Journal of Urology, 2003. 170(5): p. 2067-2071.

89. Jost, W. and P. Marsalek, Duloxetine: mechanism of action at the lower urinary tract and Onuf's nucleus. Clin Auton Res, 2004. 14(4): p. 220-7.

90. Mariappan, P., et al., Duloxetine, a serotonin and noradrenaline reuptake inhibitor (SNRI) for the treatment of stress urinary incontinence: a systematic review. Eur Urol, 2007. 51(1): p. 67-74.

91. Farmacotherapeutisch kompas. http://www.farmacotherapeutischkompas.nl.

92. Steers, W.D., et al., Duloxetine compared with placebo for treating women with symptoms of overactive bladder. BJU Int, 2007. 100(2): p. 337-45. 


\section{Chapter 8}

93. Engelmann, M., R. Landgraf, and C.T. Wotjak, The hypothalamic-neurohypophysial system regulates the hypothalamic-pituitary-adrenal axis under stress: an old concept revisited. Front Neuroendocrinol, 2004. 25(3-4): p. 132-49.

94. Arborelius, L., et al., The role of corticotropin-releasing factor in depression and anxiety disorders. J Endocrinol, 1999. 160(1): p. 1-12.

95. Nemeroff, C.B., Recent advances in the neurobiology of depression. Psychopharmacol Bull, 2002. 36 Suppl 2: p. 6-23.

96. Klausner, A.P. and W.D. Steers, Corticotropin releasing factor: A mediator of emotional influences on bladder function. Journal of Urology, 2004. 172(6 II): p. 2570-2573.

97. Robbins, M.T. and T.J. Ness, Footshock-induced urinary bladder hypersensitivity: role of spinal corticotropin-releasing factor receptors. J Pain, 2008. 9(11): p. 991-8.

98. Hanna-Mitchell, A.T., et al., Corticotropin-releasing factor family peptide signaling in feline bladder urothelial cells. J Endocrinol, 2014. 222(1): p. 113-21.

99. Imaki, T., et al., Differential regulation of corticotropin-releasing factor mRNA in rat brain regions by glucocorticoids and stress. J Neurosci, 1991. 11(3): p. 585-99.

100. Smith, A.L., et al., The effects of acute and chronic psychological stress on bladder function in a rodent model. Urology, 2011. 78(4): p. 967.e1-967.e7.

101. Klausner, A.P., et al., The role of corticotropin releasing factor and its antagonist, astressin, on micturition in the rat. Autonomic Neuroscience: Basic and Clinical, 2005. 123(1-2): p. 26-35.

102. Wood, S.K., et al., Social stress-induced bladder dysfunction: potential role of corticotropin-releasing factor. Am J Physiol Regul Integr Comp Physiol, 2009. 296(5): p. R1671-8.

103. Griffiths, D., Imaging bladder sensations. Neurourol Urodyn, 2007. 26(6 Suppl): p. 899-903.

104. Griffiths, D. and S.D. Tadic, Bladder control, urgency, and urge incontinence: Evidence from functional brain imaging. Neurourology and Urodynamics, 2008. 27(6): p. 466-474.

105. Komesu, Y.M., et al., Functional MRI of the Brain in Women with Overactive Bladder: Brain Activation During Urinary Urgency. Female Pelvic medicine and reconstructive surgery, 2011. 30(1542-5983 (Electronic)): p. 3.

106. Kerestes, R., et al., Functional brain imaging studies of youth depression: A systematic review. Neuroimage Clin, 2013. 4: p. 209-231.

107. Lederbogen, F., et al., City living and urban upbringing affect neural social stress processing in humans. Nature, 2011. 474(7352): p. 498-501.

108. Eisenberger, N.I., The neural bases of social pain: evidence for shared representations with physical pain. Psychosom Med, 2012. 74(2): p. 126-35.

109. Griffiths, D., Clinical studies of cerebral and urinary tract function in elderly people with urinary incontinence. Behav Brain Res, 1998. 92(2): p. 151-5.

110. Awata, S., et al., Regional cerebral blood flow abnormalities in late-life depression: relation to refractoriness and chronification. Psychiatry Clin Neurosci, 1998. 52(1): p. 97-105.

111. Mochizuki, H. and H. Saito, Mesial frontal lobe syndromes: correlations between neurological deficits and radiological localizations. Tohoku J Exp Med, 1990. 161 Suppl: p. 231-9.

112. Peterson, B.S., et al., Cortical thinning in persons at increased familial risk for major depression. Proc Natl Acad Sci U S A, 2009. 106(15): p. 6273-8.

113. Hyde, T.M., et al., Enuresis as a premorbid developmental marker of schizophrenia. Brain, 2008. 131(Pt 9): p. 2489-98.

114. Morgan, V., et al., Amitriptyline reduces rectal pain related activation of the anterior cingulate cortex in patients with irritable bowel syndrome. Gut, 2005. 54(5): p. 601-7.

115. Kruimel, J., et al., Integrated medical-psychiatric outpatient care in functional gastrointestinal disorders improves outcome: a pilot study. Eur J Gastroenterol Hepatol, 2015. 27(6): p. 721-7.

116. Mujagic, Z., et al., The Experience Sampling Method--a new digital tool for momentary symptom assessment in IBS: an exploratory study. Neurogastroenterol Motil, 2015. 27(9): p. 1295-302. 


Summary 
This thesis deals with the association between dysfunctions of the lower urinary tract and affective symptoms, such as anxiety and depressive symptoms. The aim of this thesis is to establish and to describe the nature of this association.

Chapter 1 provides an extensive overview of the symptoms, aetiology and treatment of lower urinary tract dysfunction. The role of the higher brain centres in the control of voiding is highlighted. In addition, the prevalence, course and pathophysiology of affective symptoms such as anxiety and depressive symptoms, are explained. Background information, from a historical perspective, is given regarding the association of lower urinary tract dysfunction and affective symptoms.

Chapter 2 describes a comprehensive qualitative overview on the association between overactive bladder ( $O A B$ ) and affective symptoms. Using validated instruments, 43 articles reporting 37 studies were included, comprising more than 80,000 subjects in total. Of these articles 32 concern depression, 2 are solely dealing with anxiety and 9 discuss both. Regarding depression and overactive bladder there is a positive association in 26 studies. Regarding anxiety 6 studies reveal a positive association. In longitudinal studies, different directional associations were reported: a) OAB patients who develop depression/anxiety or b) depressed/anxious patients who develop $O A B$, or c) both.

Chapter 3 presents the results from a cross sectional cohort study of patients with pelvic floor dysfunctions (PFDs) from our academic pelvic care centre. The prevalence of anxiety and depressive symptoms, assessed with the Hospital Anxiety and Depression Score (HADS) was high. In this cohort of 1862 patients with pelvic floor dysfunctions, such as voiding complaints, urinary and faecal incontinence and prolapse, 30.9\% had anxiety and $20.3 \%$ reported depressive symptoms. Linear (dummy-) regression analysis of HADS scales was performed to test the effects of relevant clinical predictors to pelvic floor problems, and demographic characteristics of the patients. Corrected for confounding factors, PFDs may account for $7.4 \%$ of the variance in anxiety scores and $12 \%$ of the variance in depression scores.

Chapter 4 explores the differences in bladder sensations between patients with overactive bladder syndrome and healthy volunteers by evaluating self-consciousness, self-awareness, affective complaints and sensation related bladder diaries (SR-BD). In this prospective, observational study comparing 68 patients with 66 volunteers, significant differences in SR-BD are described. Patients had lower voided volumes (193 $\mathrm{ml}$ vs. $270 \mathrm{ml}, \mathrm{p}<0.05)$, higher urinary frequency (10.6 vs. 6.6, $\mathrm{p}<0.05)$ and higher urgency scores $(2.0$ vs. $1.2,(p<0.05)$ than volunteers, while the perceived bladder fullness was similar. The self-consciousness scores were similar, but the self-awareness was significantly higher for patients ( 53.6 vs. $44.6, p<0.05$ ). This may indicate that patients with overactive bladder may attribute different values to bodily sensations than volunteers. In addition patients showed significantly higher scores for anxiety and depression according to the HADS.

Chapter 5 investigates the association between affective symptoms and conventional urodynamic parameters in 74 patients with urinary frequency. A significant association is 
seen between the total anxiety and depression score and the presence of detrusor overactivity (DO). In addition, an association is revealed between a raised anxiety score and DO as well as between a raised depression score and the sensation of urgency.

Chapter 6 discusses the predictive value of affective symptoms in the outcome of sacral neuromodulation (SNM). Eighty-six patients that underwent SNM for either OAB (66 patients) or non-obstructive retention (NOR) (20 patients) and filled out a HADS questionnaire, were included in this retrospective analysis. Significantly more OAB patients had an abnormal HADS score, compared to patients with NOR. In this preselected patient group, success of SNM treatment could not be predicted by the HADS score.

Chapter 7 describes the patient related outcome (PRO) and satisfaction regarding urinary incontinence (UI) one year after the end of treatment in our multidisciplinary pelvic care centre. Only $15.6 \%$ of the patients presented with UI only, most patients had two or three pelvic floor dysfunctions simultaneously, such as urinary incontinence, pelvic organ prolapse and constipation or faecal incontinence. A telephone survey was performed in 647 patients with UI, mainly women, one year after the end of treatment. One out of five patients with urinary incontinence recovered completely and more than one out of four stopped using incontinence absorption pads. One out of three patients went from having a multifactorial health problem to a mono-factorial health problem. High satisfaction rates were reported by more than four out of five patients. In addition, a discrepancy was seen between patient-reported cure (20.6\%) and patients that no longer required pads (27.6\%).

Chapter 8 is a general discussion in which the results and discussion of all articles is discussed in the context of current literature. It is hypothesised that a common pathway of both dysfunctions of the lower urinary tract and affective complaints may exist. Comparisons with functional complaints along the "gut-brain-axis" are made. Data regarding shared biological pathways and anatomical overlap in lower urinary tract dysfunction and affective complaints are presented.

To conclude, future perspectives are presented stating that it is very important to determine the role of stress and psychological factors on bladder function. There is need for an adequate assessment of symptom screening that overcomes the limitation of currently available methods. The consideration that psychological factors can be a possible cause of dysfunctions of the lower urinary tract, will improve diagnosis and treatment of these disorders. 

Nederlandse samenvatting 

Dit proefschrift beschrijft de relatie tussen stoornissen van de lage urinewegen en affectieve klachten, zoals angst en depressie. Het eerste doel van dit proefschrift is het verband te onderzoeken tussen angst/depressie en blaasklachten. Het tweede doel is de relatie tussen deze factoren verder te analyseren en te verklaren.

De functie van de blaas is de opslag van urine en het ledigen van de inhoud, op een gekozen en geschikte tijd en plaats. Over het algemeen zijn mensen zich gedurende de dag niet bewust van hun blaas. Op het moment dat de blaas gevoeld wordt, gaat er een signaal naar de hersenen en wordt men zich ervan bewust dat ze moeten plassen. Als zowel het moment als de plaats geschikt zijn, dan gaat er een signaal terug naar de blaas wat de lediging in gang zet. De blaasspier contraheert, terwijl de sluitspier gelijktijdig relaxeert. Vrijwillige mictie is een complex proces waarbij meerdere parallel werkende netwerken van perifere zenuwbanen, het ruggenmerg en diverse centra in de hersenen betrokken zijn.

Bij patiënten met het overactieve blaas syndroom (OAB) werkt dit normale mechanisme van opslag en lediging niet goed en is er sprake van een toegenomen mictiefrequentie met abnormale aandrang, met of zonder urineverlies. De schatting is dat ongeveer $17 \%$ van de westerse volwassenen last heeft van $O A B$ en dat dit percentage zal stijgen in de toekomst, vanwege de vergrijzing en een bekende toename van deze klachten met de leeftijd. Het overactieve blaas syndroom kan veroorzaakt worden door neurologische afwijkingen of door afwijkingen in de blaas zoals infecties, maar meestal is de oorzaak onbekend (idiopathische $O A B$ ). Voor het stellen van de diagnose gebruiken we op dit moment sensatie-gerelateerde plasdagboeken, waarin behalve de frequentie en volume van de mictie, ook het gevoel van aandrang genoteerd wordt. Verder kan ook een urodynamisch onderzoek verricht worden. Dit is een invasieve meetmethode om de druk in de blaas te bepalen middels druksensoren. Deze sensoren worden tijdens het onderzoek in de blaas gebracht en vervolgens wordt de blaas gevuld. Bij $\mathrm{OAB}$ zien we vaak, maar niet altijd, drukverhoging tijdens de vulfase van de blaas, hetgeen duidt op een overactiviteit van de blaasspier (detrusor overactiviteit, DO).

De oorzaak van $O A B$ is grotendeels onbekend en de huidige therapieën voor $O A B$ gericht op de blaas hebben niet altijd het gewenste resultaat. Derhalve rijst de vraag of er slechts één of meerdere mechanismen zijn de symptomen van OAB kunnen verklaren. Psychische factoren kunnen de blaasfunctie ook beïnvloeden, zoals blijkt uit bijvoorbeeld het feit dat veel mensen vaker naar het toilet moeten als ze stress ervaren.

Angst en depressieve klachten zijn veelvoorkomend in de westerse wereld en in het verleden is al een relatie beschreven tussen psychiatrische ziekten en de blaas. Ook in de huidige literatuur is er een verband aangetoond tussen affectieve symptomen en blaasklachten, echter over deze relatie is nog erg weinig bekend. 


\section{DIT PROEFSCHRIFT BEVAT ACHT HOOFDSTUKKEN:}

Hoofdstuk 1 is een algemene inleiding over de verschillende disfuncties van de lage urinewegen. Behalve OAB worden ook inspanningsincontinentie, plasklachten en bekkenbodemklachten besproken. De anatomie en fysiologie van de lage urinewegen wordt uitgelegd, met een duidelijk accent op de rol van de hogere hersencentra op de controle van de mictie. Verder geeft dit hoofdstuk een toelichting op de aard en het ontstaan van affectieve klachten. Het eerste hoofdstuk wordt afgerond door een uiteenzetting te geven over de relatie tussen affectieve klachten en blaasklachten, vanuit historisch perspectief.

De huidige literatuur over de associatie tussen OAB en affectieve klachten worden beschreven in hoofdstuk 2. Alleen studies die gevalideerde meetmethoden hebben gebruikt, worden gepresenteerd. Bij deze systematische zoektocht heeft ons team in totaal 43 relevante artikelen gevonden, die 37 studies beschrijven met in totaal meer dan 80.000 mensen. Het merendeel van deze artikelen heeft betrekking op depressieve klachten, en een kleiner aantal bespreekt angst in relatie tot blaasklachten. Ook zijn er artikelen die beiden bespreken. Er zijn 26 studies die een positieve associatie tonen tussen depressieve klachten en $O A B$ en 6 studies tonen een positieve associatie tussen angst en $\mathrm{OAB}$. Longitudinale studies laten verbanden in verschillende richtingen zien: a) patiënten met $O A B$ ontwikkelen later in de tijd affectieve klachten of b) patiënten met affectieve klachten ontwikkelen later in de tijd OAB of c) beiden.

Hoofdstuk 3 geeft de prevalentie weer van angst en depressieve klachten binnen de groep patiënten met bekkenbodemdisfunctie die gezien zijn in ons academisch bekkenbodemcentrum. Dit centrum behandelt patiënten met klachten van de bekkenbodem, zoals incontinentie voor urine of ontlasting, mictieklachten en verzakkingsklachten. In deze groep van 1862 patiënten had 30.9\% angstklachten en 20.3\% depressieve klachten. Dit is vergelijkbaar met scores bij patiënten met kanker of cardiale klachten en hoger dan scores gevonden bij patiënten met diabetes. Statistische analyse, met correctie voor beïnvloedende factoren, wijst uit dat $7.4 \%$ van de angstscores en $12 \%$ van de depressiescores verklaard kunnen worden door de bekkenbodemklachten.

De vraag of er verschil is in blaassensaties tussen patiënten met $O A B$ en gezonde vrijwilligers, wordt beantwoord in Hoofdstuk 4. Er is in een prospectief onderzoek gekeken naar verschillen in zelfbewustzijn, affectieve klachten en sensatie-gerelateerde plasdagboeken tussen 68 patiënten en 66 vrijwilligers. Patiënten plassen vaker en met kleinere gemiddelde hoeveelheden, maar met hogere graden van aandrang dan vrijwilligers. Het blaasvullingsgevoel was echter gelijk. De score op de zelfbewustzijn vragenlijst die iets zegt over iemands karakter met betrekking tot zelfbewustzijn, was gelijk, terwijl de score op de situationele zelfbewustzijn vragenlijst significant hoger was voor patiënten ten opzichte van vrijwilligers. Dit kan betekenen dat patiënten met OAB anders reageren op lichaamssensaties dan gezonde vrijwilligers en er zich meer bewust 
van zijn. Daarnaast hadden patiënten een duidelijk hogere score op angst en depressieve klachten gemeten met de HADS (Hospital Anxiety and Depression Scale) vragenlijst.

De associatie tussen affectieve klachten en het urodynamisch onderzoek worden weergegeven in Hoofdstuk 5. Er werd een positieve associatie gevonden tussen de totale HADS score en DO bij patiënten met frequency. Tevens is er een associatie tussen een verhoogde angstscore en DO en tussen een verhoogde depressiescore en urgency.

Behandeling van disfunctie van de lage urinewegen met sacrale neuromodulatie (SNM) is niet altijd effectief en de vraag werd gesteld of affectieve symptomen de uitkomst van SNM kunnen voorspellen. Bij SNM wordt geprobeerd blaascontrole te herstellen door modulatie van opstijgende zenuwprikkels en blaasreflexen in het centraal zenuwstelsel, middels een bij de sacrale zenuwwortel gelegen electrode die verbonden is met een onderhuidse pacemaker. In Hoofdstuk 6 worden de resultaten getoond van SNM in relatie tot de HADS scores. SNM werd toegepast voor niet-obstructieve retentie (onvermogen tot plassen) bij 20 patiënten en voor OAB bij 66 patiënten. Patiënten met OAB klachten hadden hogere HADS scores vergeleken met de retentiepatiënten. Binnen deze groep voorgeselecteerde patiënten, bij wie de behandelindicatie al gesteld was, kon de uitkomst van de SNM behandeling echter niet worden voorspeld door de HADS score.

De door de patiënt gerapporteerde uitkomsten (patient related outcome, PRO) en tevredenheid met betrekking tot urine incontinentie na behandeling in ons multidisciplinaire bekkenbodemcentrum worden gerapporteerd in Hoofdstuk 7. Het merendeel van de patiënten presenteerde zich met twee of drie bekkenbodemproblemen tegelijkertijd, bijvoorbeeld verzakkingsklachten, incontinentie voor ontlasting of obstipatie, terwijl maar $15.6 \%$ van de patiënten zich meldden met alleen urine incontinentie.

$\mathrm{Er}$ is een jaar na het einde van de behandeling een telefonische enquête uitgevoerd bij 647 patiënten, voornamelijk vrouwen. Eén op de vijf patiënten met urine incontinentie was compleet genezen en één op de vier was gestopt met het gebruik van incontinentiemateriaal. Verder was één op de drie patiënten van een multifactorieel bekkenbodem probleem naar één bekkenbodemprobleem gegaan. Vier op de vijf patiënten meldden een hoge patiënttevredenheid. Er was een discrepantie tussen het percentage door de patiënten zelf aangegeven genezing (20.6\%) en het percentage van patiënten bij wie geen verbandmateriaal meer nodig was (27.6\%).

Het proefschrift wordt afgerond in Hoofdstuk 8 met een algemene discussie waarin alle studies die voorkomen in dit proefschrift besproken worden in de context van de huidige literatuur. De recente literatuur bevestigt dat er voldoende bewijs bestaat voor de associatie tussen depressieve klachten en $O A B$, en in mindere mate voor angst en $\mathrm{OAB}$. Er is mogelijk een gemeenschappelijk oorzakelijk traject dat zowel disfunctie van de urinewegen als ook affectieve klachten kan verklaren. De verschillende hypotheses die deze associatie kunnen verklaren, worden gepresenteerd. Er kan sprake zijn van overlappende biochemische trajecten en anatomische overlap tussen blaasdisfuncties en affectieve klachten. Zo zijn dezelfde neurotransmitters en hormonen van belang bij 
zowel affectieve klachten als bij blaasklachten. Daarnaast tonen verschillende beeldvormende onderzoeken afwijkingen aan in dezelfde hersengebieden, bij zowel disfuncties van de lage urinewegen als bij angst en depressie. Ook wordt een vergelijking gemaakt met andere functionele klachten, zoals prikkelbare darm syndroom. Deze patiënten reageren ook anders op lichamelijke prikkels, evenals patiënten met OAB. In dit kader wordt het nut van psychologische screening benadrukt.

Het is zeer belangrijk dat in de toekomst de rol van stress en psychologische factoren op de blaasfunctie verder wordt onderzocht. Tevens is aanvullend onderzoek nodig naar de individuele psychische reacties op blaassensaties. Er is dan ook nood aan een adequate beoordeling van symptomen van het OAB symptoomcomplex, die de beperkingen van de huidige retrospectieve methode met plasdagboeken en vragenlijsten niet heeft. Zo kan idealiter een onderscheid worden gemaakt tussen de oorzaken van de disfunctie. Het besef dat psychologische factoren een mogelijke oorzaak kunnen zijn van disfuncties van de lage urinewegen kan de diagnose, en met name de behandeling, van deze klachten verbeteren. 
Valorisation 



\section{INTRODUCTION}

Lower Urinary Tract Dysfunction (LUTD) is a very common disorder and has a considerable impact on quality of life. Two main categories of dysfunction of the lower urinary tract exist: storage and voiding dysfunction. The storage dysfunction, the overactive bladder syndrome $(O A B)$, is the focus of this thesis. The International Continence Society (ICS) defines OAB as a symptom complex of urgency, usually with frequency and nocturia (awakening at night to void), with ( $O A B$ wet) or without (OAB dry) urgency urinary incontinence (UUI). Urgency is the complaint of a sudden compelling desire to pass urine, which is difficult to defer ${ }^{1}$. Urgency is the paramount symptom of OAB.

\section{TARGET POPULATION}

The results of this manuscript are relevant to physicians who care for patients with symptoms of LUTD such as (increased) urinary frequency, UUI, OAB, pelvic floor dysfunctions, as well as physicians who care for patients with affective complaints.

\section{EPIDEMIOLOGY OF LOWER URINARY TRACT DYSFUNCTIONS}

Approximately $17 \%$ of the adult population of the United States are estimated to be affected by $\mathrm{OAB}^{2}$. In Europe the prevalence is comparable, $16 \%$ of adults over 40 years are affected, as reported in a cross-sectional community-based study in 6 countries $^{3}$. In addition, the incidence of both $O A B$ wet and dry increases with age ${ }^{4-6}$. Therefore, due to ageing worldwide, the estimated number of adults affected with UUI is expected to increase from 55 million in 2013 to 60 million in 2018. Furthermore, it is suggested that 500 million people will have OAB in 2013 and this number would increase to 546 million people in $2018^{7}$.

\section{SOCIO-ECONOMIC RELEVANCE}

The health care costs of $O A B$ are high, as total costs are estimated to be around 12 billion USD per year and 267 USD per person with OAB in one year ${ }^{8}$. On top of that, work productivity, in terms of work interruptions, changes in work and location can be significantly impacted by $O A B$ as well ${ }^{9}$. OAB wet has greater impact on work productivity than $O A B$ dry, and more so in men than in women ${ }^{10}$.

Furthermore, $O A B$ can be associated with significant comorbidity. Urgency urinary incontinence has been associated with increased risk of falls and fractures in two large longitudinal studies ${ }^{11,12}$. It has even been mentioned, in a Finish longitudinal study, that 
urgency and UUI are independent risk factors for increased mortality, especially in older men $^{13}$.

Sexual health is affected by $O A B$ dry as well as $O A B$ wet. There is diminished sexual activity and enjoyment of sex in patients with $O A B$, compared to people without urinary symptoms ${ }^{14}$. The diminished sexual function has not only been presented in women, but also in men, as $O A B$ wet and dry are significant predictors of erectile dysfunction and ejaculatory disorders ${ }^{15}$.

As a result of this burden, $O A B$ with and without incontinence has been associated with significantly lower quality of life scores compared to matched controls without voiding complaints ${ }^{4,16}$. The degree of bother is increased with the frequency of both urgency and UUI in both men and women of all ages complaints ${ }^{17}$. As much as 32 incontinence related quality of life items have been identified in a focus group investigation. Patients tend to focus on coping with embarrassment and interference from incontinence and emotional wellbeing. ${ }^{18}$. But not only patients are affected themselves, dysfunctions of the lower urinary tract also have an impact on family members ${ }^{19}$.

Patients with $O A B$ use different coping strategies, such as timed voiding, locating toilets or avoidance of drinking, rather than consulting health care providers ${ }^{20}$. In addition, the self-management of OAB leads almost inevitably to social withdrawal, sometimes relational problems and fear in daily life, potentially leading to social and psychological problems ${ }^{21}$. Therefore, OAB can seriously affect daily life and has been compared to other chronic illnesses. Moreover, the physician tends to underestimate the patients bother of urinary symptoms ${ }^{22}$.

Although $O A B$ affects daily life and social relationships, and has an adverse impact on quality of life, many individuals with $O A B$ complaints do not bring their problems to the attention of health care providers ${ }^{23}$. This is mainly because of embarrassment ${ }^{20}$ or unawareness of medical treatment for $\mathrm{OAB}^{3}$. A very large study with 162.906 respondents in the United States reported that, of the individuals with overactive bladder symptoms, only less than half of them had discussed their symptoms with a healthcare provider. Only $22.5 \%$ had previously used medication for overactive bladder, and only $8.1 \%$ were currently on treatment ${ }^{24}$. The results of a large European interview study were similar. Only $60 \%$ of respondents with symptoms had consulted a doctor and merely $27 \%$ were currently receiving treatment ${ }^{3}$. In addition, an inventory in a managed care organization revealed that 3 out of 4 patients with OAB did not receive pharmacotherapy for symptoms ${ }^{25}$. The adherence to antimuscarinic drugs is low ${ }^{26}$. There is need for a more adequate therapy for OAB. At this moment, the assessment and treatment mainly focuses on the bladder, but it is necessary to consider a treatment focus on other possible causes of dysfunctions of the lower urinary tract.

Depression and anxiety are symptom based constructs, and classified as affective disorders according to the DSM-V psychiatric diagnostic criteria ${ }^{27}$. According to a survey of the World Health Organisation, anxiety is globally the most common psychiatric disorder ${ }^{28}$. The 12 -month prevalence of anxiety disorders in the US adult population is 
around $18 \%{ }^{28}$, and almost $10 \%$ for depression ${ }^{29}$. Additionally, the US-life time prevalence is approximately 30\% for anxiety disorders, and 20\% for depressive disorders ${ }^{30}$. However, according to the World Mental Health survey almost half of the individuals with serious complaints received no treatment in the twelve months prior to the diagnostic interview ${ }^{31}$. In general, the association of somatic diseases with a depressive disorder is not uncommon and affects $25 \%$ of the people in hospital in-patient populations $^{32}$.

For instance, in irritable bowel syndrome (IBS), a functional disorder of intestinal hypersensitivity and altered motility, the association with affective disorders is relatively clear, and IBS patients have been shown to have an approximately $60 \%$ higher odds to suffer from depression ${ }^{33}$. In addition, half of the population reporting a lifetime IBS diagnosis also had a lifetime mood or anxiety disorder ${ }^{34}$. Nevertheless psychiatric comorbidity regarding IBS is often unrecognized ${ }^{35}$. Moreover, at least in complex patients with medically unexplained functional complaints, psychiatric comorbidity is frequently under-detected ${ }^{36}$. This thesis states that there is an association between LUTD and affective symptoms. In turn, anxiety and depressive symptoms can intensify UI and decrease the benefit of treatment ${ }^{37}$.

\section{FUTURE}

The consideration that psychological factors, as discussed in this manuscript, can be a possible cause or lead to deterioration of lower urinary tract dysfunction can enable physicians to improve diagnosis and to identify patients who will benefit from a multimodal treatment. Therefore, it will be very important to distinguish the physical and psychological causes of the dysfunction and their relative contribution and interaction. The data in this manuscript are currently used for the development of a new diagnostic tool that overcomes the limitations of currently available methods. This tool, which consists of randomly, repeated momentary assessments in everyday life, may provide a better overview of the aetiology of the symptoms. Hence, it can indicate potential triggers that may influence symptom development. Hopefully, in the future we can select patients with LUTD who may benefit from (additional) psychological of psychiatric treatment. 


\section{REFERENCES}

1. Abrams, P., et al., The standardisation of terminology in lower urinary tract function: report from the standardisation sub-committee of the International Continence Society. Urology, 2003. 61(1): p. 37-49.

2. Coyne, K.S., et al., National community prevalence of overactive bladder in the United States stratified by sex and age. Urology, 2011. 77(5): p. 1081-7.

3. Milsom, I., et al., How widespread are the symptoms of an overactive bladder and how are they managed? A population-based prevalence study. BJU Int, 2001. 87(9): p. 760-6.

4. Stewart, W.F., et al., Prevalence and burden of overactive bladder in the United States. World J Urol, 2003. 20(6): p. 327-36.

5. Irwin, D.E., et al., Population-Based Survey of Urinary Incontinence, Overactive Bladder, and Other Lower Urinary Tract Symptoms in Five Countries: Results of the EPIC Study. European Urology, 2006. 50(6): p. 1306-1315.

6. Coyne, K.S., et al., The prevalence of lower urinary tract symptoms (LUTS) in the USA, the UK and Sweden: results from the Epidemiology of LUTS (EpiLUTS) study. BJU Int, 2009. 104(3): p. 352-60.

7. Irwin, D.E., et al., Worldwide prevalence estimates of lower urinary tract symptoms, overactive bladder, urinary incontinence and bladder outlet obstruction. BJU Int, 2011. 108(7): p. 1132-8.

8. Hu, T.W., et al., Estimated economic costs of overactive bladder in the United States. Urology, 2003. 61(6): p. 1123-8.

9. Sexton, C.C., et al., Impact of overactive bladder on work productivity in the United States: results from EpiLUTS. Am J Manag Care, 2009. 15(4 Suppl): p. S98-S107.

10. Irwin, D.E., et al., Impact of overactive bladder symptoms on employment, social interactions and emotional well-being in six European countries. BJU Int, 2006. 97(1): p. 96-100.

11. Brown, J.S., et al., Urinary incontinence: does it increase risk for falls and fractures? Study of Osteoporotic Fractures Research Group. J Am Geriatr Soc, 2000. 48(7): p. 721-5.

12. Foley, A.L., et al., Association between the Geriatric Giants of urinary incontinence and falls in older people using data from the Leicestershire MRC Incontinence Study. Age Ageing, 2012. 41(1): p. 35-40.

13. Vrijens, D., et al., Affective symptoms and the overactive bladder - A systematic review. J Psychosom Res, 2015;78:95-108.

14. Heidler, S., et al., Impact of overactive bladder symptoms on sexuality in both sexes. Urol Int, 2010. 85(4): p. $443-6$.

15. Coyne, K.S., et al., The impact of OAB on sexual health in men and women: results from EpiLUTS. I Sex Med, 2011. 8(6): p. 1603-15.

16. Liberman, J.N., et al., Health-related quality of life among adults with symptoms of overactive bladder: results from a U.S. community-based survey. Urology, 2001. 57(6): p. 1044-50.

17. Vaughan, C.P., et al., The prevalence of clinically meaningful overactive bladder: bother and quality of life results from the population-based FINNO study. Eur Urol, 2011. 59(4): p. 629-36.

18. DuBeau, C.E., et al., The impact of urge urinary incontinence on quality of life: importance of patients' perspective and explanatory style. J Am Geriatr Soc, 1998. 46(6): p. 683-92.

19. Coyne, K.S., L.S. Matza, and J. Brewster-Jordan, "We have to stop again?!": The impact of overactive bladder on family members. Neurourology and Urodynamics, 2009. 28(8): p. 969-975.

20. Ricci, J.A., et al., Coping strategies and health care-seeking behavior in a US national sample of adults with symptoms suggestive of overactive bladder. Clin Ther, 2001. 23(8): p. 1245-59.

21. Nicolson, P., et al., It's just the worry about not being able to control it! A qualitative study of living with overactive bladder. Br J Health Psychol, 2008. 13(Pt 2): p. 343-59.

22. Rodriguez, L.V., et al., Discrepancy in patient and physician perception of patient's quality of life related to urinary symptoms. Urology, 2003. 62(1): p. 49-53.

23. Epstein, L.B. and R.P. Goldberg, The overactive bladder and quality of life. International journal of fertility and women's medicine, 2005. 50(1): p. 30-36. 
24. Benner, J.S., et al., Bother related to bladder control and health care seeking behavior in adults in the United States. J Urol, 2009. 181(6): p. 2591-8.

25. Randolph, M.F. and M. Greenfield, Lower urinary tract obstruction in normal male children. Early detection by urinary diary. Am J Dis Child, 1965. 110(5): p. 523-30.

26. Lucas MG, B.D., et al. EAU Guidelines on Urinary Incontinence. 2014; Available from: http://www.uroweb.org/guidelines/online-guidelines/. .

27. Diagnostic and Statistical Manual of Mental Disorders. 5 ed. 2013, Washington, DC: American Psychiatric Association.

28. Wang, P.S., et al., Twelve-month use of mental health services in the United States: results from the National Comorbidity Survey Replication. Arch Gen Psychiatry, 2005. 62(6): p. 629-40.

29. Ustun, T.B., et al., Global burden of depressive disorders in the year 2000. Br J Psychiatry, 2004. 184: p. 386-92.

30. Kessler, R.C., et al., Lifetime prevalence and age-of-onset distributions of DSM-IV disorders in the National Comorbidity Survey Replication. Arch Gen Psychiatry, 2005. 62(6): p. 593-602.

31. Demyttenaere, K., et al., Prevalence, severity, and unmet need for treatment of mental disorders in the World Health Organization World Mental Health Surveys. JAMA, 2004. 291(21): p. 2581-90.

32. Kroenke, K., Patients presenting with somatic complaints: epidemiology, psychiatric comorbidity and management. Int J Methods Psychiatr Res, 2003. 12(1): p. 34-43.

33. Cole, J.A., et al., Migraine, fibromyalgia, and depression among people with IBS: a prevalence study. BMC Gastroenterol, 2006. 6: p. 26.

34. Mykletun, A., et al., Prevalence of mood and anxiety disorder in self reported irritable bowel syndrome (IBS). An epidemiological population based study of women. BMC Gastroenterol, 2010. 10: p. 88.

35. Pinto-Sanchez, M.I., et al., Anxiety and Depression Increase in a Stepwise Manner in Parallel With Multiple FGIDs and Symptom Severity and Frequency. Am J Gastroenterol, 2015. 110(7): p. 1038-48.

36. Leue, C., et al., Managing complex patients on a medical psychiatric unit: an observational study of university hospital costs associated with medical service use, length of stay, and psychiatric intervention. J Psychosom Res, 2010. 68(3): p. 295-302.

37. Bogner, H.R., et al., Anxiety disorders and disability secondary to urinary incontinence among adults over age 50. Int J Psychiatry Med, 2002. 32(2): p. 141-54. 

Dankwoord 

Hier heb ik me al lang op verheugd: dat ik mijn dankwoord mag gaan schrijven, maar nu blijkt het toch wel een lastig stukje te zijn. Het is immers één van de best gelezen onderdelen van een proefschrift en natuurlijk wil ik niemand vergeten. Ik wil iedereen die, op welke manier dan ook, heeft bijgedragen aan de totstandkoming van dit proefschrift, heel hartelijk danken. Er is echter een groot aantal mensen die ik speciaal wil noemen.

Ik wil beginnen mijn promotieteam te bedanken.

Professor dr. Ph. Van Kerrebroeck, beste Philip, dankjewel voor je steun en begeleiding, zowel bij het proefschrift als ook op de werkvloer. Je bent gestart als mijn begeleider als keuze coassistent en later was je mijn opleider. Nu ben je voor mij een mentor van wie ik veel leer, maar met wie ik ook veel kan lachen. Een aantal jaren geleden belde je me, tijdens een moeilijke periode voor mij, met de vraag of ik bij de urologie wilde komen werken. Ik geloof dat ik je nooit heb laten weten hoe belangrijk dat voor me was, bij dezen alsnog van harte bedankt, voor mij maakte dat een wereld van verschil.

Professor dr. G. van Koeveringe, beste Gommert, hartelijk bedankt voor je kritische blik en de leermomenten. Niet alleen op onderzoeksgebied, maar ook in de functionele urologie. Het is prettig met je samen te werken. Ik kan altijd bij je binnen lopen en om advies vragen en ik stel de feed back die ik van je krijg erg op prijs. Je hebt me geleerd geen problemen maar kansen te zien en niet te klein te denken.

Dr. C. Leue, beste Carsten, het is een verrijking voor mij te zien dat je patiënten met urologische klachten ook vanuit een ander blikveld kan benaderen. Ik heb veel mensenkennis van je geleerd en gezien dat je mensen in een kort consult heel goed kunt duiden, daarom vind ik nauw samenwerken met een psychiater toch een beetje eng. Ik ben dan ook aan het wachten op mijn diagnose.

Graag wil ik de leden van de beoordelingscommissie prof.dr. Steinbusch (voorzitter), prof. dr. Lycklama à Nijeholt, prof. dr. Nijhuis, prof. dr. De Ridder en dr. Strik hartelijk danken voor de tijd en moeite die u heeft gestoken in het lezen en beoordelen van dit proefschrift.

Natuurlijk wil ik ook al mijn medeauteurs bedanken. Jamie Drossaerts; mede door jouw nauwkeurigheid en planmatig werken is het een goede review geworden. Ondanks (of misschien wel dankzij) onze verschillende werkwijzen was onze samenwerking meer dan prima. Ik waardeer het geduld dat je met me hebt gehad als ik weer eens met een computer vraag kwam. Het zal niet lang meer duren dan is jouw proefschrift klaar, veel succes met de laatste loodjes.

Dr. B. Berghmans, beste Bary, hartelijk dank voor je enthousiasme en optimisme, maar ook voor je geduld als ik iets niet begreep. Het was fijn dat we het eens konden zijn dat we het oneens waren. Het heeft in ieder geval onze samenwerking niet in de weg gestaan. 
Dr. Nieman, hartelijk dank voor het uitvoeren van ingewikkelde statistiek, het was een onmisbare bijdrage voor het prevalentie artikel.

Dr. Marcelissen, beste Tom, ik had je gevraagd eens kritisch naar het artikel over zelfbewustzijn te kijken, maar toen ik het terugkreeg met ongezouten kritiek en er niet veel van het oorspronkelijke artikel overbleef, had ik er bijna spijt van. Bijna, want uiteindelijk is het een beter artikel geworden. Dankjewel voor je kritische blik, bij dit artikel en tijdens de laatste fase van mijn proefschrift. Het is een plezier met je samen te werken en ik hoop dat we samen nog een aantal artikelen kunnen schrijven.

Prof. dr. de Wachter, beste Stefan, Dr. Heeringa, beste Rhea en Vera Venneman, hartelijk dank voor het voorwerk waar ik verder op mocht borduren.

Hartelijk dank aan (destijds) studenten die een waardevolle bijdrage hebben geleverd aan diverse artikelen. Jolanda Spakman, Martijn Smits, Sam Degaillier en Isabel Schilders heel erg bedankt voor jullie inzet.

Het gehele onderzoeksteam binnen de functionele urologie in Maastricht wil ik bedanken voor het fijne onderzoeksklimaat. Een aantal van ons team wil ik apart bedanken: $\mathrm{Dr}$ Hohnen, beste Ramona, het ICS congres in Tokyo zal ik niet snel vergeten. Ik wens je veel succes met je nieuwe baan. Ranjana Jairam, dank voor je adviezen en succes met de laatste fase van je proefschrift. Dr Rahnamai'i, beste Sajjad, hartelijk dank voor de waardevolle tips rondom promoveren. Kevin Rademakers, bedankt voor de inspirerende gesprekken over onderzoek, je bent de volgende die aan de beurt is om te promoveren en ik twijfel er niet aan dat je het heel goed zult doen.

Zeker wil ik ook alle vrijwilligers en patiënten bedanken die onder andere geheel belangeloos meerdere dagen de moeite hebben genomen om plasdagboekjes en vragenlijsten in te vullen. Daarbij extra dank voor Karin Weber voor de enthousiaste ondersteuning bij de inclusie.

Alle collega's van het Pelvic Care Centrum Maastricht wil ik bedanken voor de medebeoordeling van alle patiënten en natuurlijk de plezierige samenwerking binnen ons team. De dames Patricia Bloem, Bianca Hambeukers en Marion Bosch voor het afnemen van de vragenlijsten en de goede patiëntenzorg.

Het secretariaat, lieve Edith, Nancy, Anja en Shelly, hartelijk dank voor de ondersteuning en de nodige afleiding bij een kopje thee. Fijn dat jullie steeds meeleven.

Alle medewerkers van de poli en functieafdeling van de urologie merci voor het opsturen van de HADS vragenlijsten en het doen van de urodynamische onderzoeken. Het is zeer aangenaam met jullie allen samen te werken.

Marjo Kleijnen dank voor je huidige en toekomstige inzet voor een goede patiëntenzorg. 
Alle (oud) assistenten van de afdeling urologie, het geeft energie samen te werken met jonge en leergierige collega's, dankjewel hiervoor. Ik hoop dat ik mijn enthousiasme voor onderzoek op jullie kan overbrengen.

Uiteraard wil ik ook de overige mannen (naast de promotoren) van de urologie bedanken, Kees van de Beek, Piet Callewaert, dr. Elmer Francisca, prof. dr. Mathias Oelke en dr. Joep van Roermund. Hartelijk dank voor de prettige werksfeer en de getoonde interesse. Ik vind het altijd zeer gezellig als jullie komen buurten in mijn hokje. De mannendag heeft me inspiratie voor een stelling opgeleverd, waarvoor dank. Gelukkig kunnen we, naast het serieuze werk, ook regelmatig met elkaar lachen.

Carlo Le Sage en Paul Mohr dank voor de waardevolle gesprekken en het zien van promoveren in het juiste perspectief.

Gregorey hartelijk dank voor het tekenen van de omslag. Erg fijn dat je het zo enthousiast hebt opgepakt. Ik waardeer het geduld dat je met me hebt gehad en jouw flexibiliteit op mijn last minute verzoeken.

Dr. Rehrer, beste Nancy, bij jou ben ik het eerst met onderzoek in aanraking gekomen, hartelijk dank voor die geweldige stage.

Dr. Oerlemans, beste Dennis, jouw promotie begin dit jaar was voor mij een goede leerschool. Hartelijk dank voor al je adviezen en hulp bij het laatste stukje van het promotietraject. Fijn dat ik steeds weer met mijn vragen bij je terecht kon.

Beste vrienden, en in het bijzonder Frank, René, Roger, Monique, John, Nicole, Frank, Audrey, Davy, Corine, Bram, Sandra, Fons, Inge, Babette, Romain, Ralf, Marijke en Patrick, vrienden in goede en slechte tijden. Hartelijk dank voor het aanhoren van mijn promotieperikelen, maar vooral dank voor jullie vriendschap.

Heel groot woord van dank aan mijn paranimfen:

Lieve Saskia en lieve Sylvia, vanaf de eerste onderwijsgroep van de studie geneeskunde zijn we vriendinnen. Een hechte en warme vriendschap die me heel veel waard is. We hebben het diepste verdriet maar gelukkig ook heel veel vreugde samen gedeeld. Ik ben er trots op dat jullie mijn paranimfen willen zijn, het geeft een fijn gevoel te weten dat jullie bij de verdediging zowel letterlijk als figuurlijk achter me staan.

Mam, pap en Peggy, hartelijk dank voor het warme nest en de fijne basis. Jullie hebben mij veel geleerd en altijd gesteund in mijn keuzes. Ik kan altijd bij jullie terecht en dat is een heel groot goed.

Leo, mijn dank voor jou laat zich niet op papier zetten.

Als laatste wil ik Seb, Lina en Emi bedanken en mijn verontschuldigingen aanbieden. Dit boekje is voor een groot deel aan de keukentafel ontstaan, tussen de Lego en de puzzels met de autosafe op 5 minuten, omdat er dan weer een handje op het toetsenbord 
kwam. Sorry dat mama vaak aan haar boekje moest werken. Lieve Seb, dankjewel dat je er bent en dat je altijd heel enthousiast reageert en er echt voor gaat zitten om vragen te stellen. Hoe was je dag mama? Wat heb je gedaan? Wat voor een boekje is dat? Wat is dat een promotie? Ik ben heel erg trots op je. Lieve Lina, mijn lief gevoelig meisje dat altijd zo goed wil helpen. Je hebt me zeker geholpen. Ik wil je mijn excuses aanbieden dat je dit jaar op een andere datum jarig bent. Lieve Emi, jij bent nog te klein om het te snappen, maar levendig genoeg om afleiding te geven. Lieve Seb, Lina en Emi, dank voor jullie enthousiasme en levendigheid. Als ik alleen al aan jullie denk, moet ik lachen. Bedankt dat jullie er zijn. 


\section{Curriculum Vitae}

Désirée (Daisy) Vrijens is geboren op 2 oktober 1974 te Maastricht. Ze behaalde haar VWO diploma in 1993 aan het Sint-Maartenscollege te Maastricht. In datzelfde jaar startte ze de studie geneeskunde aan de Universiteit van Maastricht. Het derde jaar van haar studie heeft ze in Engeland, aan de University of Bristol gevolgd. Haar eerste kennismaking met onderzoek was tijdens haar wetenschapsstage 'Plasma sodium concentration during exercise in the heat' aan de University of Otago, in Dunedin,

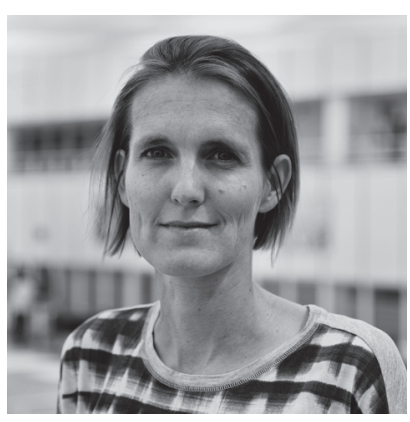
Nieuw-Zeeland.

Na het behalen van haar artsdiploma in 1999 heeft ze kortdurend gewerkt als bedrijfsarts alvorens een half jaar door Zuidoost Azië te gaan fietsen. Na deze rondreis was ze opnieuw werkzaam in de bedrijfsgeneeskunde, vervolgens in de chirurgie en de kaakchirurgie. Hierna kwam ze als arts assistent niet in opleiding urologie te werken in het azM. De opleiding tot uroloog begon ze in 2004 met de vooropleiding algemene heelkunde in het toenmalige Atrium MC te Heerlen (nu Zuyderland MC) met als opleiders dr.C.J.van der Linden en dr. R.J.Th.J. Welten. Het academische deel van haar opleiding heeft ze in het Maastricht UMC+ gevolgd met prof. dr. Ph.E.V.A Van Kerrebroeck als opleider. Zij heeft haar opleiding tot uroloog afgerond in het Atrium MC onder supervisie van dr. K.P.J. Delaere.

Na haar opleiding is ze in 2010 als chef de clinique gaan werken in het Atrium MC. $\mathrm{Na}$ een periode als uroloog in Maastricht UMC+ en vervolgens als waarnemer in het Wilhelmina Ziekenhuis in Assen heeft zij sinds 2013 een aanstelling als staflid in Maastricht UMC+. Ze heeft een deelaanstelling aan de Universiteit Maastricht voor onderwijs. Haar klinische aandachtsgebieden zijn functionele urologie en neuro-urologie. In de zomer van 2013 startte zij haar promotieonderzoek 'Dysfunctions of the lower urinary tract and affective symptoms' onder begeleiding van prof.dr.Ph.E.V.A Van Kerrebroeck en prof.dr.G.A.van Koeveringe (promotores) en dr.C.Leue (co-promotor). De afgelopen jaren heeft ze haar taken binnen het onderwijs en patiëntenzorg gecombineerd met promotieonderzoek.

Ze woont samen met Leo van Houten. Ze is trotse mama van vier kinderen: Seb (2010), Lina (2012), Emi (2014) en John (†2010). 



\section{Lijst van publicaties}

Vrijens D, Berghmans B, Nieman F, van Os J, van Koeveringe G, Leue C. Prevalance of anxiety and depressive symptoms and their association with pelvic floor dysfunctions- $A$ cross sectional cohort study at a Pelvic Care Centre. Neurourol Urodyn. 2017 feb 21[Epub ahead of print].

Leue C, Kruimel J, Vrijens D, Masclee A, van Os J, van Koeveringe G. Functional urological disorders: a sensitized defence response in the bladder-gut-brain axis. Nat Rev Urol. 2017 Mar;14(3):153-163.

Vrijens D, Drossaerts J, Rademakers K, Smits M, de Wachter S, Leue C, van Koeveringe G.Associations of Psychometric Affective Parameters with Urodynamic Investigation for Urinary Frequency. Low Urin Tract Symptoms 2016 Jun 12[Epub ahead of print].

Drossaerts J, Vrijens D, Leue C, Schilders I, Van Kerrebroeck P, van Koeveringe G.Screening for depression and anxiety in patients with storage or voiding dysfunction: $A$ retrospective cohort study predicting, outcome of sacral neuromodulation. Neurourol Urodyn. 2016 Nov;35(8):1011-1016.

Vrijens D, Spakman J, van Koeveringe G, Berghmans B. Patient-reported outcome after treatment of urinary incontinence in a multidisciplinary pelvic care clinic. Int J Urol. 2015 Nov; 22(11):1051-7.

Vrijens D, Drossaerts J, van Koeveringe G, Van Kerrebroeck P, van Os J, Leue C. Affective symptoms and the overactive bladder - a systematic review. J Psychosom Res. 2015 Feb; 78(2):95-108.

Callewaert P, Rahnama'j M, Guimarães M, Vrijens D, Van Kerrebroeck P.Double Longitudinal Megapreputium Incision Technique: the dolomite. Urology 2014 May; 83(5):114954.

Vrijens D, Rehrer N. Sodium-free fluid ingestion decreases plasma sodium during exercise in the heat. J appl Physiol. 1999 Jun; 86(6):1847-51. 



\section{List of abbreviations}

\begin{tabular}{|c|c|}
\hline $5-\mathrm{HT}$ & Serotonin \\
\hline ACC & Anterior Cingulate Cortex \\
\hline ACG & Anterior Cingulate Gyrus \\
\hline $\mathrm{ACTH}$ & Adrenocorticotropic Hormone \\
\hline BPS & Bladder Pain Syndrome \\
\hline CPPS & Chronic Pelvic Pain Syndrome \\
\hline CRF & Corticotrophin Releasing Factor \\
\hline $\mathrm{dACC}$ & dorsal part of the Anterior Cingulate Cortex \\
\hline DO & Detrusor Overactivity \\
\hline ESM & Experience Sampling Methodology \\
\hline $\mathrm{FI}$ & Faecal incontinence \\
\hline HADS & Hospital Anxiety and Depression scale \\
\hline HADS-A & Hospital Anxiety and Depression scale anxiety sub-score \\
\hline HADS-D & Hospital Anxiety and Depression scale depression sub-score \\
\hline HPA & Hypothalamus Pituitary Adrenal \\
\hline IBS & Irritable Bowel Syndrome \\
\hline ICS & International Continence Society \\
\hline IPSS & International Prostate Symptom Score \\
\hline LUTD & Lower Urinary Tract Dysfunctions \\
\hline LUTS & Lower Urinary Tract Symptoms \\
\hline LUTS & Lower Urinary Tract Symptoms \\
\hline MBSR & Mindfulness-Based Stress Reduction \\
\hline MUI & Mixed Urinary Incontinence \\
\hline n.a. & non applicable \\
\hline NE & Norepinephrine \\
\hline NOR & Non-obstructive urinary retention \\
\hline OAB & Overactive Bladder Syndrome \\
\hline OAB dry & Overactive Bladder Syndrome without Urgency Urinary Incontinence \\
\hline OAB wet & Overactive Bladder Syndrome with Urgency Urinary Incontinence \\
\hline PAG & Periaqueductal Grey \\
\hline PCC & Pelvic Care Center \\
\hline PFDs & Pelvic Floor Dysfunctions \\
\hline PMC & Pontine Micturition Centre \\
\hline POP & Pelvic Organ Prolapse \\
\hline PRO & Patient-Reported Outcome \\
\hline PROMs & Patient-Reported Outcome Measures \\
\hline QoL, & Quality of Life \\
\hline
\end{tabular}


SCS

s.d.

SNM

SR-BD

SSAS

SSRI

SUI

UDS

UI

UUI

VAS
Self-Consciousness Scale

standard deviation

Sacral Neuromodulation

Sensation-Related Bladder Diary

Situational Self-Awareness Scale

Selective Serotonin Reuptake Inhibitor

Stress Urinary Incontinence

Urodynamic Study

Urinary Incontinence

Urgency Urinary Incontinence

Visual Analogue Scale 

Publication of this thesis is financially supported by:

Stichting Wetenschappelijke Activiteiten Maastrichtse Urologie, Allergan, Astellas Pharma bv, Coloplast, ChipSoft, Ferring Pharmaceuticals, Goodlife Pharma, Laborie, Medireva, Pierre Fabre Santé. Pohl Boskamp bv, Zambon.

No company has influenced the content of this thesis. 
